\title{
A State Space Odyssey
}

\section{The Multiplex Dynamics of Cardiac Arrhythmias}

\author{
Dissertation \\ for the award of the degree \\ "Doctor rerum naturalium" \\ of the Georg-August-Universität Göttingen
}

within the doctoral program "Göttingen Graduate School for Neurosciences, Biophysics, and Molecular Biosciences" (GGNB)

of the Georg-August University School of Science (GAUSS)

submitted by

Thomas Lilienkamp

from Minden

Göttingen, 2017 


\section{Members of the Thesis Committee:}

apl. Professor. Dr. Ulrich Parlitz

Research Group Biomedical Physics

Max Planck Institute for Dynamics and Self-Organization, Göttingen, Germany

Professor Dr. Andreas Tilgner

Institute of Geophysics

Georg-August-Universität, Göttingen, Germany

Dr. Florian Rehfeldt

Third Institute of Physics - Biophysics

Georg-August-Universität, Göttingen, Germany

Professor Dr. Christoph Schmidt

Third Institute of Physics - Biophysics

Georg-August-Universität, Göttingen, Germany

Members of the Examination Board:

apl. Professor. Dr. Ulrich Parlitz (First Reviewer)

Research Group Biomedical Physics

Max Planck Institute for Dynamics and Self-Organization, Göttingen, Germany

Professor Dr. Andreas Tilgner (Second Reviewer)

Institute of Geophysics

Georg-August-Universität, Göttingen, Germany

Further members of the Examination Board:

Dr. Florian Rehfeldt

Third Institute of Physics - Biophysics

Georg-August-Universität, Göttingen, Germany

Professor Dr. Christoph Schmidt

Third Institute of Physics - Biophysics

Georg-August-Universität, Göttingen, Germany

Professor Dr. Jens Frahm

Biomedical NMR Research

Max Planck Institute for Biophysical Chemistry, Göttingen, Germany

Professor Dr. Reiner Kree

Institute for Theoretical Physics

Georg-August-Universität, Göttingen, Germany

Date of oral examination: January 17, 2018 
I hereby declare that this thesis has been written independently and with no other sources and aids than quoted.

Thomas Lilienkamp 
"There is nothing like looking, if you want to find something. You certainly usually find something, if you look, but it is not always quite the something you were after."

- J.R.R. Tolkien 


\begin{abstract}
With three million people worldwide (three hundred thousand people in the United States alone) experiencing sudden cardiac arrest per year, it is one of the most common causes of death in developed countries. Ventricular fibrillation, a dysfunction of the heart characterized by a highly chaotic spatio-temporal wave dynamics, is the main cause for sudden cardiac arrest. The application of a high-energy defibrillation shock, as the current medical treatment to restore the sinus rhythm, comes along with severe side-effects, among others additional damage of the heart. Furthermore, patients with an ICD (implantable cardioverter-defibrillator) in particular suffer from posttraumatic stress symptoms.

The goal of this thesis is to investigate the dynamics of the heart (and in particular the nature of cardiac arrhythmias (specifically ventricular fibrillation)) using concepts and perceptions from the dynamical systems theory. On the basis of the interdisciplinary interplay between mathematical approaches and interaction with experimental and clinical knowledge and results, two general scientific objectives are addressed:
\end{abstract}

- Derive an enhanced understanding of the dynamics during episodes of ventricular fibrillation, including the development of concepts for the improvement of current defibrillation techniques and suggestions for completely new strategies which may find their way into the clinical application.

- Obtain novel insights into the fundamental dynamics of complex, nonlinear systems (thus excitable systems and beyond).

These objectives are addressed using numerical simulations, which constitute the main tool to investigate specific research questions. The results of this thesis are organized in four chapters, each focusing on one specific question:

- The first results chapter is dealing with the mechanism of spontaneous termination of ventricular fibrillation. We investigate the transient behavior of spiral and scroll wave dynamics using different cell models. The observed transients can be classified into the group of so called type-II supertransients. We find, that in 3D simulations, a critical thickness of the medium plays an essential role. Basic features of the simulations agree with general observations of clinicians, e.g. that larger heart muscle volumes increase the risk of cardiac arrhythmias.

- In the second results chapter, we address the question whether a self-termination of a chaotic episode can be predicted. By applying small but finite perturbations to specific trajectories of chaotic spiral wave dynamics we find that the state space structure close to the "exits" of the chaotic regime changes significantly. We could verify this effect also in low-dimensional maps. This analysis shows, that although the upcoming self-termination is not visible in conventional variables, it should in principle be possible to derive such a quantity. 
- In the third results chapter, we investigate complexity fluctuations of the chaotic spatiotemporal dynamics in simulations using realistic heart geometries. We show, that the level of organization of the spatio-temporal dynamics can be estimated by analyzing the time series of a multi-electrode setup.

- In the last results chapter, we discuss whether a successful termination of chaotic spiral wave dynamics is possible using a minimal interaction with the system. We show, that since the underlying topological object which determines the chaotic dynamics is a chaotic saddle, one can terminate the dynamics (as a proof of concept) by the application of a specific but very small perturbation.

We hope that the insights provided by this thesis contribute to the general understanding of cardiac arrhythmias and the nonlinear dynamics of complex systems. The results suggest that an improved medical treatment of cardiac arrhythmias can benefit from:

- A more detailed state analysis of the dynamics during spatio-temporal chaos, incorporating diverse measure techniques (e.g. multiple-ECG measurements, CT scans, MRI scans).

- An intervention strategy which should adapt to individual patients and the respective dynamical state of the heart.

A variety of new experimental approaches will be available which may help to achieve these goals and to improve the understanding of the phenomena investigated in this thesis:

Filament identification in the bulk tissue during experiments using sophisticated ultra sound techniques, inverse ECG measurements for the reconstruction of spatio-temporal wave dynamics or using techniques from optogenetics for the stimulation of cardiac tissue via light pulses are promising candidates which can have a significant impact on the field of cardiac dynamics. This technological progress in combination with novel data analysis techniques from the fields of machine learning or data assimilation and sophisticated simulations of the complex dynamics has great potential to develop advanced and efficient strategies for a patient specific medical treatment. 


\section{Contents}

$\begin{array}{lll}1 & \text { Introduction } & 1\end{array}$

1.1 How Does the Heart Work? . . . . . . . . . . . . . . . . . . . . . . 1

$1.2 \quad$ Cardiac Arrhythmias $\ldots \ldots \ldots \ldots \ldots \ldots$

1.2 .1 Ventricular Fibrillation . . . . . . . . . . . . . 5

1.2 .2 The Risk of Ventricular Fibrillation . . . . . . . . . . . . . . 6

1.2 .3 Defibrillation of the Heart $\ldots \ldots \ldots \ldots \ldots$

1.3 Dynamical Systems and Nonlinear Dynamics $\ldots \ldots \ldots \ldots$. . . . . . . 8

1.4 The Scope of this Thesis $\ldots \ldots \ldots \ldots \ldots$. . . . . . . . . . . . 9

\begin{tabular}{lll}
\hline 2 & Methods & 11
\end{tabular}

2.1 Complexity of the Heart . . . . . . . . . . . . . . . . . . . . 11

2.1.1 Single Cell Dynamics - Cardiac Tissue as an Excitable Medium. . . . 11

$2.1 .2 \quad$ Signal Propagation on Different Scales . . . . . . . . . . . . . . . . . . . . . 17

2.1 .3 Mechanical Contraction . . . . . . . . . . . . . . . 20

2.1 .4 The Electrocardiogram . . . . . . . . . . . . . . . . . . . . . . . . . . . . .

2.1 .5 Defibrillation of the Heart . . . . . . . . . . . . . . . 22

2.2 Mathematical Embedding . . . . . . . . . . . . . . . . . . . 25

2.2 .1 The Heart as a Dynamical System … . . . . . . . . . 25

2.2 .2 Structures of the State Space $\ldots \ldots \ldots \ldots$. . . . . . . . . . . . . . . . . . . . 26

2.2 .3 Transient Chaos . . . . . . . . . . . . . . . . 30

2.2 .4 Analyzing Chaotic Dynamics . . . . . . . . . . . . . . 34

2.3 The Role of Numerical Simulations . . . . . . . . . . . . . . . . . . . . . . . . 40

$2.3 .1 \quad$ Interplay of Experiments and Numerical Simulations . . . . . . . . 40

2.3 .2 The Process of Modeling . . . . . . . . . . . . . . . . . . 41

2.3 .3 Designing a Simulation . . . . . . . . . . . . . . . . . . 42

2.4 Numerical Simulations . . . . . . . . . . . . . . . . . . . . . . . . . . . . 43

2.4 .1 From Bidomain to Monodomain Model . . . . . . . . . . . . . . 44

2.4 .2 Models of Local Cell Dynamics . . . . . . . . . . . . . . . . . . . 48

$2.4 .3 \quad$ Discretization and Stability . . . . . . . . . . . . . . . . . . . . . . . . . . 52

2.4 .4 Boundary Conditions . . . . . . . . . . . . . . . . . 57

2.4 .5 Analyzing Tools . . . . . . . . . . . . . . . . . 6 60

2.4 .6 Software Package MediaSim . . . . . . . . . . . . . . . . . . . 65

2.4 .7 The Hardware . . . . . . . . . . . . . . . . . . . . . . . . 66 
\begin{tabular}{llr}
\hline 3 & Results & 67
\end{tabular}

3.1 Features of Chaotic Transients in Excitable Media . . . . . . . . . . . . . 68

3.2 Terminal Transient Phase of Chaotic Transients . . . . . . . . . . . . . . 76

$3.2 .1 \quad$ Introduction $\ldots \ldots \ldots \ldots \ldots \ldots \ldots \ldots$

3.2 .2 Methods . . . . . . . . . . . . . . . . . . . 79

3.2 .3 Results $\ldots \ldots \ldots \ldots$. . . . . . . . . . . . . . . . . . . 81

3.2 .4 Conclusion $\ldots \ldots \ldots \ldots$

3.3 Complexity Fluctuations during Ventricular Fibrillation . . . . . . . . . . . 102

3.3 .1 Introduction . . . . . . . . . . . . . . . . . . 104

3.3 .2 Methods . . . . . . . . . . . . . . . . . . . . . 105

3.3 .3 Results . . . . . . . . . . . . . . . . . . . 107

3.3 .4 Conclusion . . . . . . . . . . . . . . . . . . . . . . . 114

3.4 Termination via Perturbation . . . . . . . . . . . . . . . . . . . . 117

3.4 .1 General Concept . . . . . . . . . . . . . . . . . . . . 118

3.4 .2 Implementation of the Algorithm . . . . . . . . . . . . . 120

3.4.3 Results . . . . . . . . . . . . . . . . . . . . . . . . . . . 121

\begin{tabular}{|lr|}
4 Conclusion & 125 \\
\hline
\end{tabular}

4.1 Discussion and Outlook . . . . . . . . . . . . . . . . . . . 125

4.2 Concluding Remarks $\ldots \ldots \ldots$. . . . . . . . . . . . . . . 130

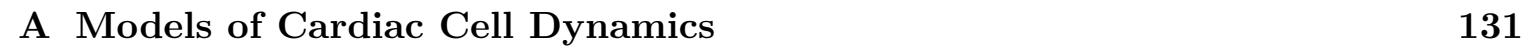

A.1 Aliev-Panfilov Model . . . . . . . . . . . . . . . . . . . . . . . . . . . . . . . . 131

A.2 Fenton-Karma Model . . . . . . . . . . . . . . . . . . . . . . . . 132

A.3 Bueno-Orovio-Cherry-Fenton Model . . . . . . . . . . . . . . . . . 133

\begin{tabular}{ll}
\hline B Supplemental Material & $\mathbf{1 3 5}$
\end{tabular}

B.1 Supplemental Material 1 . . . . . . . . . . . . . . . . . . . . . . . 135

B.2 $\quad$ Supplemental Material 2 . . . . . . . . . . . . . . . . . . . . . . . . . . . . . . . . . . . . . . . . . . . . . .

B.3 Supplemental Material 3 . . . . . . . . . . . . . . . . . . . . . . . 147

\begin{tabular}{lr}
\hline List of Figures & 159
\end{tabular}

\begin{tabular}{ll}
\hline List of Tables & 163
\end{tabular}

\begin{tabular}{ll}
\hline Bibliography & 165
\end{tabular} 


\section{Abbreviations}

APD action potential duration. 16

AV atrioventricular. 19

CT computed tomography. 23

DRC dose response curve. 24

ECG Electrocardiogram. 21

FDM finite-difference method. 53

FEM finite element method. 58

NFIL number of filaments. 109

NPS number of phase singularities. 156

PDE partial differential equation. 149

PE Permutation Entropy. 106

PSI Phase Synchronization Index. 115

RMSD root mean squared deviation. 115

SA sinoatrial. 19

TTP Terminal Transient Phase. 81

VF Ventricular Fibrillation. 106 


\section{Chapter 1}

\section{Introduction}

Research in physics advances in three broad domains: The first and foremost, astronomy and relativity, concerning the infinitely big; quantum mechanics and particle physics, dealing with the infinitely small; and the domain of infinitely complex encompassing the Physics of Biological and Complex Systems (PBCS). Third Infinity focuses on the third infinite domain of science inspired by the well-known chaotic double pendulum problem to understand life processes at a quantitative and molecular level.

Motto of the (biannual) "Third Infinity Conference on Physics of Biological and Complex Systems' $₫$ in Göttingen.

${ }^{a}$ Organized by PhD students. Last conference: October 9-11 2017.

In the second part of the 20th century, the role of interdisciplinarity in natural sciences increased significantly. Where previously specific theories, models, and methods were often developed for one particular field only (e.g. physics, chemistry, biology), one profits nowadays more and more from a fruitful exchange of concepts and ideas. In today's research, clear boundaries between formerly separated disciplines are often smeared out: methods or strategies developed in e.g. mathematical physics are also used in more applied fields (like medicine, ecology or environmental sciences).

The aim of this thesis is to extend the general understanding of the functionality of the heart, in particular to elucidate the underlying mechanisms of cardiac arrhythmias, by using concepts and methods from physics and mathematics. Referring to the citation at the beginning of this chapter, this interaction of disciplines is maybe the only solution to achieve a broad and thorough understanding of a system which is as complex as the heart.

\subsection{How Does the Heart Work?}

The heart is an essential organ of humans and many animals, which pumps the blood through the body and provides in this way the supply of oxygen and nutrients for the cells of the organism. In the human body, the blood circulation is divided into the pulmonary circuit and the systemic circuit (Fig. 1.1), also called the peripheral circulation and the greater circulation. The first one is related to (and driven by) the right atrium and ventricle 


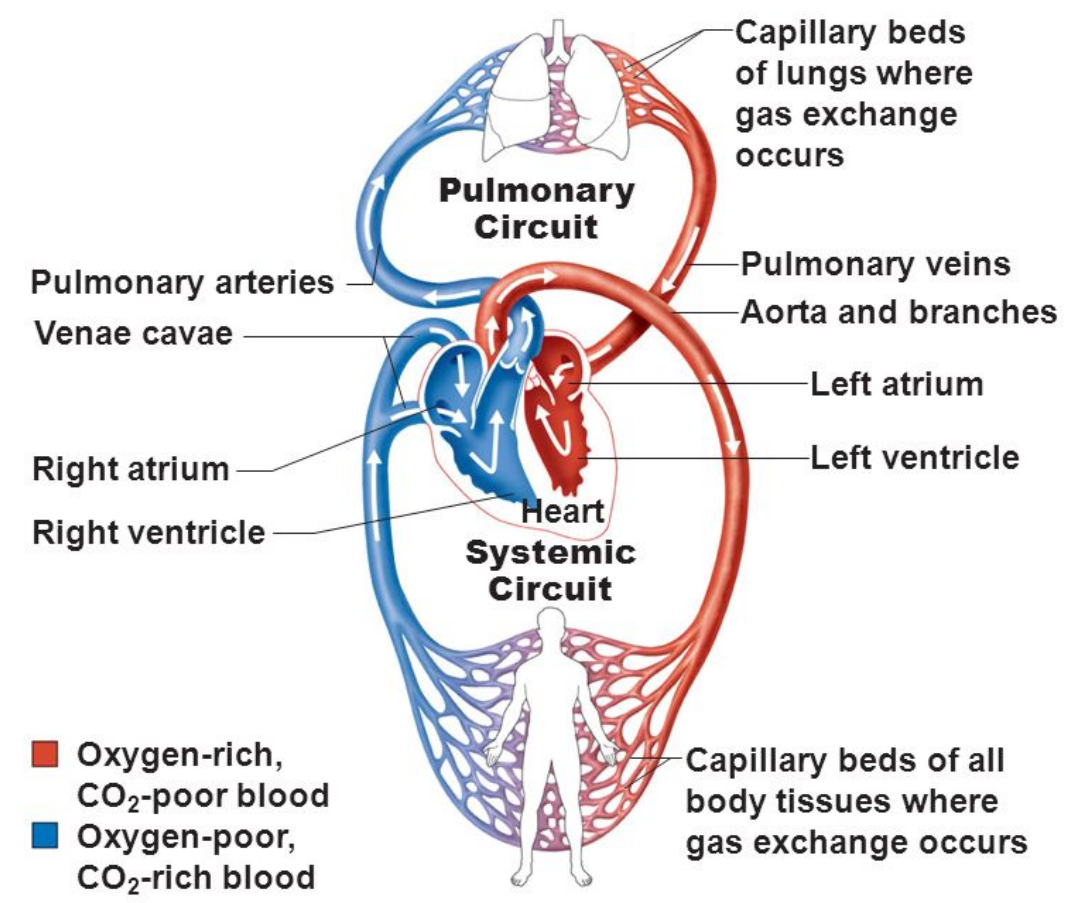

Figure 1.1: The circulation system in the human body. The oxygen-poor blood is pumped from the right ventricle through the pulmonary arteries to the lungs where the gas exchanges happens. The oxygen-rich blood returns to the heart by the pulmonary veins and enters the left atrium of the heart pulmonary circuit. The systemic circuit is responsible for the transportation of the oxygen-rich blood from the heart (left ventricle) through the aorta and its branches to the tissue of the body. The oxygen-poor blood returns subsequently through the venae cavae back to the heart (right atrium). Reprinted from [1] by permission of Pearson Education, Inc., New York, New York.

of the heart, whereas the left atrium and ventricle pump the blood through the systemic circuit.

The main purpose of the pulmonary circuit is to transport the blood to the lungs, in order to release carbon dioxide from the blood and to absorb oxygen. Subsequently, the systemic circuit delivers the blood (with a high oxygen concentration) to the rest of the body, and also returns the blood with a larger concentration of carbon dioxide back to the heart (where it enters the pulmonary circuit, and so on). The heart is the organ which drives this circuit constantly by its pumping function as long as the body is alive.

One distinguishes between the left and right part of the heart: the blood enters the left and right atria through the pulmonary veins (left) and the inferior and superior venae cavae (right), before it is pumped to the left and right ventricle (Fig. 1.2), respectively. The mitral valve (left) and the tricuspid valve (right) prevent that blood flows back from the respective ventricles into the atria. Finally, the blood leaves the ventricles, passing the aortic valve (left) and the pulmonary valve (right) and entering the aorta (systemic circuit) and the pulmonary artery (pulmonary circuit), respectively. 


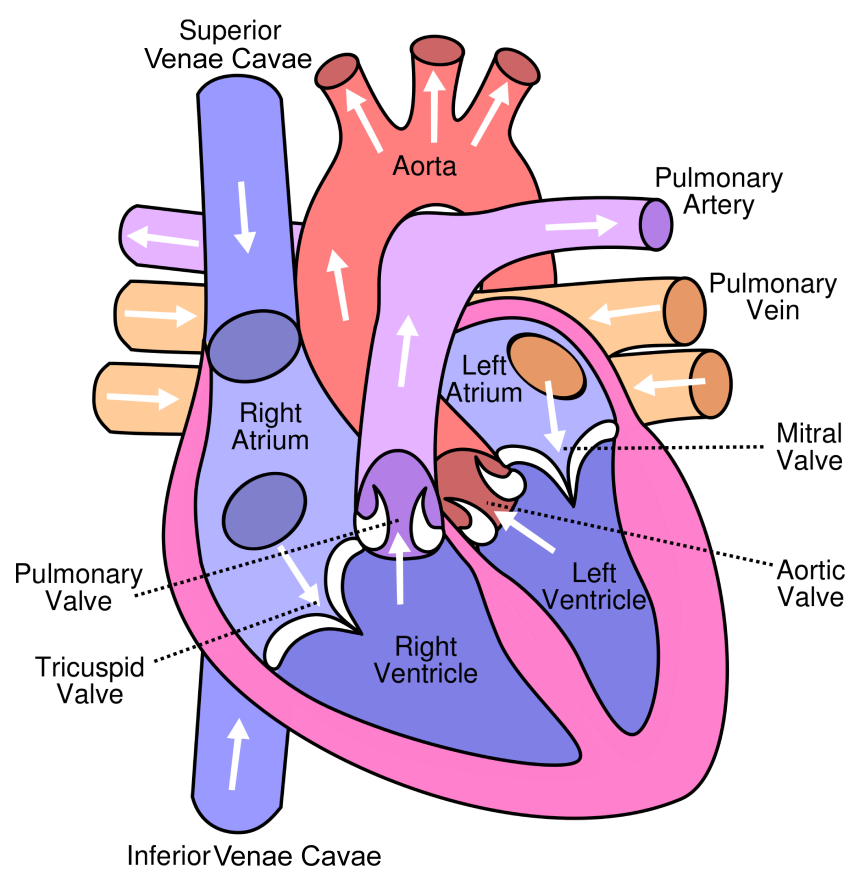

Figure 1.2: The anatomy of the heart. The heart is composed of the right and left atrium, as well as the corresponding right and left ventricle. The blood flow between the atria and the ventricles is controlled by the tricuspid valve, and the mitral valve, respectively for the right and left part. The blood leaves the right ventricle through the pulmonary artery and enters the right atrium via the venae cavae. The corresponding blood vessels for the left part of the heart are the pulmonary veins (entering the left atrium) and the aorta (leaving the left ventricle). Image reprinted from Wikimedia Commons [2].

The wall thickness differs in humans considerably between atria ( $1 \mathrm{~mm}$ to $2 \mathrm{~mm}$ ) [3] and the ventricles ( $5 \mathrm{~mm}$ to $6 \mathrm{~mm}$, end diastolic) [4, which are separated by the interventricular septum. Furthermore, one distinguishes three layers of the heart, the endocardium (innermost layer), the middle myocardium (the cardiac muscle) and the epicardium (outer layer). During a contraction the muscle tissue expands, whereas the total volume of the heart remains approximately constant. Thus, the expansion is directed to the inside, and in this way the blood is pumped out of the heart (Fig. 1.3). Since the systemic circuit is more demanding in terms of the pumping performance, the ventricles are not symmetric, but the left ventricle is usually larger (in terms of wall thickness), and the right ventricle is aligned around the more central left ventricle.

The normal, periodic contraction of the heart is also called sinus rhythm. The actual mechanical contraction is triggered by an electrical signal, which propagates through the tissue (details about the mechanism and the electro-mechanical coupling will be discussed later).

\section{Separation of Electrical and Mechanical Dynamics}

A general assumption which is also used in this thesis exploits the approximately unidirectional relation between the electrical signal and the subsequent mechanical contraction. The basic idea is, when studying the dynamics of the heart, specifically in the case of a 


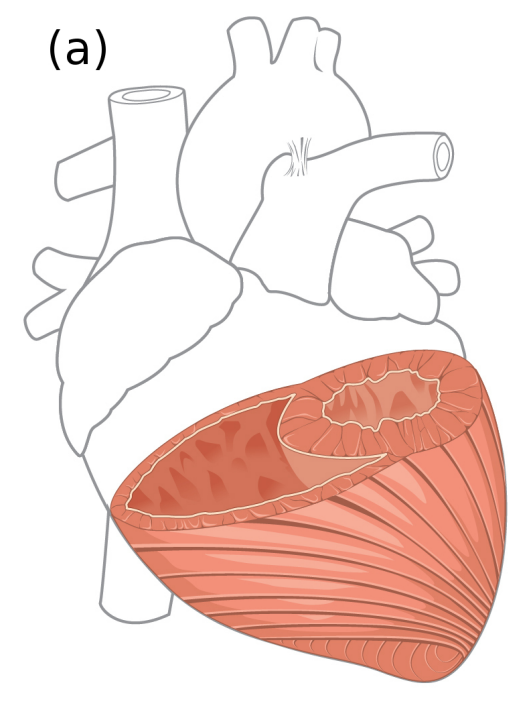

Relaxed

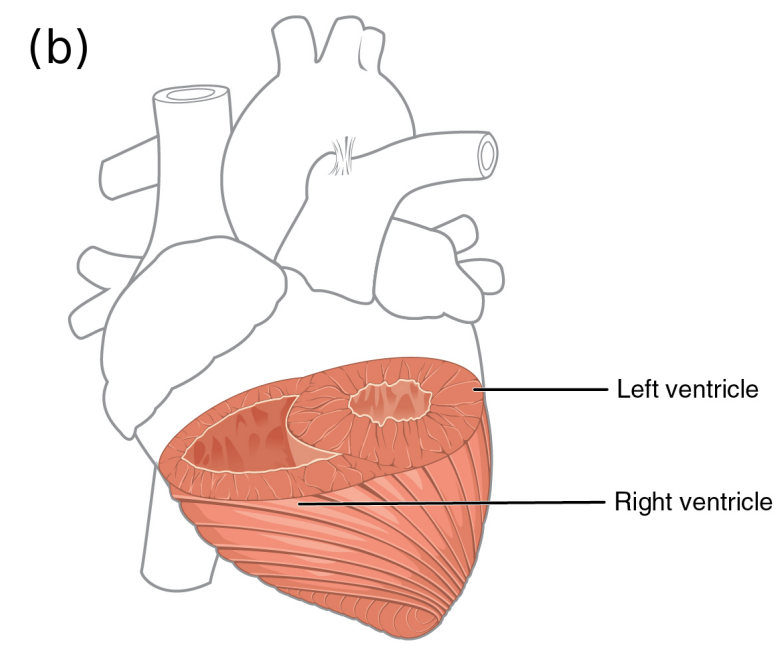

Contracted

Figure 1.3: The contraction of the heart muscle. The figure sketches the qualitative differences of the muscle volume of the ventricles between the relaxed state (a) and the contracted state (b). Image reproduced from [5].

failing heart function, it is enough to understand and control the electrical dynamics, since it directly triggers the mechanical contraction. This concept is used in experiments (e.g. in many cases the mechanics is uncoupled from the electrical dynamics, in order to facilitate the analysis of the electrical wave dynamics) and also in numerics, where often only the electrical wave propagation is simulated. Actually, also medical treatments like the application of a defibrillation shock, relates to the electrical properties of the system.

Although, a coupling in the other direction (from mechanics to electric) is possible (due to stretch activated channels [6]), we neglect this effect in all studies of this thesis and assume that the mechanical contraction of the cells is a passive property of the tissue, which is triggered by the electrical signal.

\subsection{Cardiac Arrhythmias}

Conditions of the heart where the heart beat deviates from the usual sinus rhythm (faster, slower or irregular), are called arrhythmias. These abnormalities can have various causes, for example anatomical changes of the heart (e.g. scar tissue, induced by a myocardial infarction), or other disorders of the electrical conduction system (e.g. Wolff-Parkinson-White syndrome [7]). These arrhythmias deviate in their level of impairment of the proper pumping function and the directly related health risk.

Ventricular fibrillation is the most dangerous cardiac arrhythmia, characterized by an unorganized electrical and therefore also mechanically irregular dynamics of the heart, instead of an organized periodic contraction (as during sinus rhythm). In this state the pumping function is significantly reduced, leading after a few minutes to sudden cardiac death. In the 
(a)

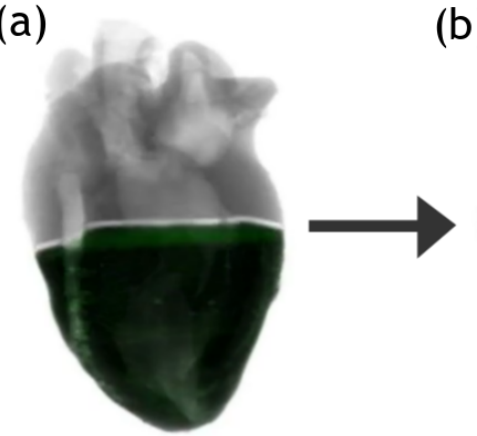

(b)

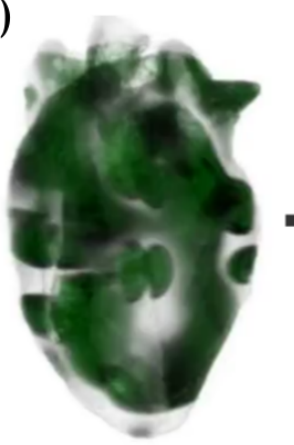

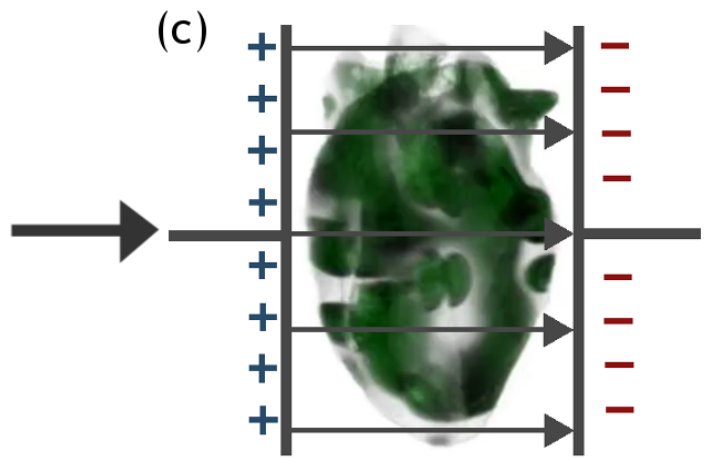

Figure 1.4: The difference of the spatio-temporal dynamics between sinus rhythm and ventricular fibrillation and the principle of defibrillation. The usual sinus contraction can be associated with a plane wave ((a), excited tissue is depicted in green) propagating from the bottom to the top of the heart. Instead, the dynamics during ventricular fibrillation is determined by scroll waves (b), which overdrive the slower sinus rhythm. In order to restore the sinus rhythm, an electrical field is applied (c), which aims at terminating the chaotic spatio-temporal dynamics inside the heart (details about the governing processes here will be discussed in 2.1 .5 on page 22 . In practice, the electrodes which generate the electrical field are arranged differently (e.g. electrode patches attached to the skin of the body in the case of external defibrillation). Simulation by Sebastian Stein.

next section, the dynamics during ventricular fibrillation is described in more detail, before risk factors are discussed and the standard medical treatment (application of a defibrillation shock) is depicted including its side-effects.

The lack of understanding of the underlying mechanism driving ventricular fibrillation and the search for alternative therapies with significant improvements of today's medical treatment (specifically a reduction of the severe side-effects) provide the main motivation for this thesis.

\subsubsection{Ventricular Fibrillation}

With three million people worldwide (three hundred thousand people in the United States alone) experiencing sudden cardiac arrest per year, it is one of the most common causes of death in developed countries. Actually, more people in the United States die from sudden cardiac arrest than from AIDS, breast cancer, lung cancer and stroke combined [8]. Ventricular fibrillation is the main cause for sudden cardiac arrest [7].

The electrical dynamics which governs the mechanical contraction of the heart deteriorates from a periodic and organized pattern, to spatio-temporal chaos ${ }^{1}$ determined by spiral or scroll waves (Fig. 1.4). These waves represent the main building blocks of the dynamics and their frequency is faster $(\gtrsim 5 \mathrm{~Hz})$ than the usual sinus rhythm $(\sim 1 \mathrm{~Hz})$. For this reason, the common stimulation which initiates the sinus rhythm is overdriven by the much faster frequency of the chaotic dynamics.

\footnotetext{
${ }^{1}$ In this thesis, chaos is used in the sense of deterministic chaotic behavior, which is characterized by a sensitive dependence on initial conditions, exponential divergence of initially close trajectories and the resulting incapability of reliable long-term predictions.
} 


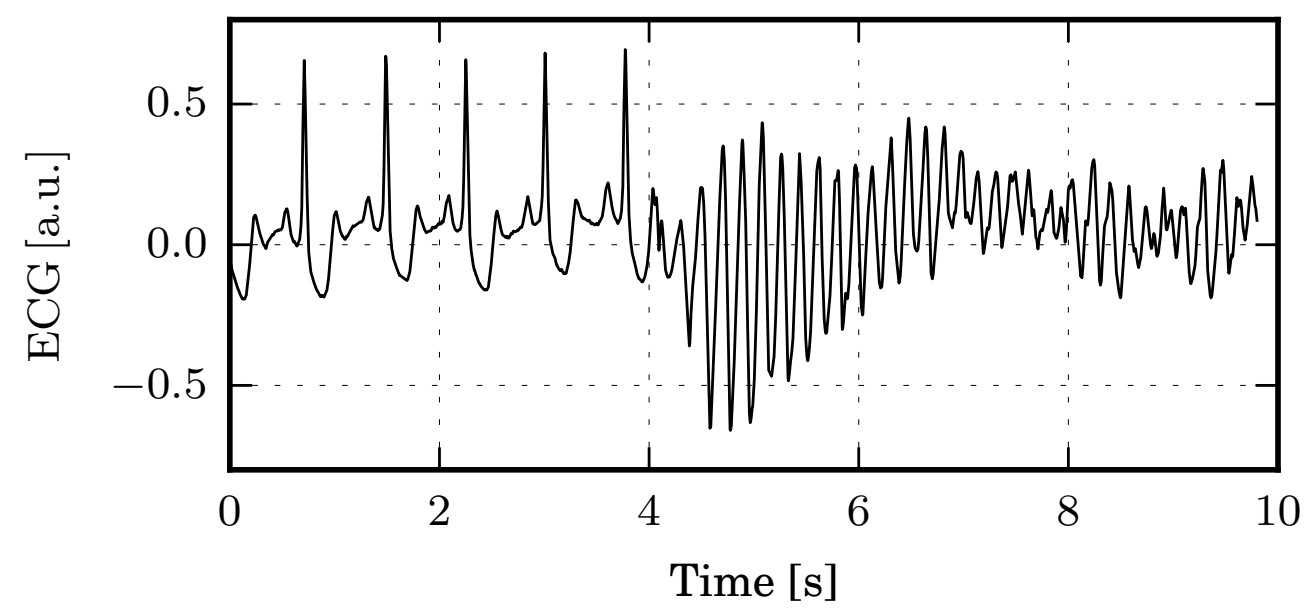

Figure 1.5: The transition from sinus rhythm to ventricular fibrillation recorded by an electrocardiogram. The figure depicts an electrocardiogram (ECG) of a male patient (43 years old). The shape of the ECG changes over time from the typical shape associated with sinus rhythm (up to $4 \mathrm{~s}$ ) to a chaotic signal (from $8 \mathrm{~s}$ ) corresponding to ventricular fibrillation. ECG time series data taken from [14].

In many cases, the transition from sinus rhythm to ventricular fibrillation is not direct but an intermediate state, called ventricular tachycardia, can be identified. This state is characterized by a still periodic dynamics, but in comparison to the sinus rhythm it exhibits a significantly increased frequency (greater than $2 \mathrm{~Hz}[9]$ ). The heart can remain in this state, but may also decay into ventricular fibrillation. Although alternan ${ }^{2}$ is a reasonable mechanism which could explain the dynamical transition from ventricular tachycardia to ventricular fibrillation [10, 11, 12, 13, the exact mechanisms responsible for the onset and perpetuation of ventricular fibrillation remain unclear.

The transition from sinus rhythm to ventricular fibrillation is also measurable in the electrocardiogram (ECG) (Fig. 1.5), as a change from a periodic to a chaotic signal.

\subsubsection{The Risk of Ventricular Fibrillation}

In clinical studies, the risk for the occurrence of ventricular fibrillation has been statistically related to other diseases or measurable biomarkers. Among others, the following risk factors have been identified [15]:

- Coronary artery disease

- Heart failure and/or decreased left ventricular ejection fraction

- Previous events of sudden cardiac arrest

- Hypertrophic cardiomyopathies

- Long QT syndrome

\footnotetext{
${ }^{2}$ Alternans describes a phenomenon where the electrical activity of the heart and the resulting strength of the mechanical contraction undergo a periodic beat-to-beat oscillation.
} 


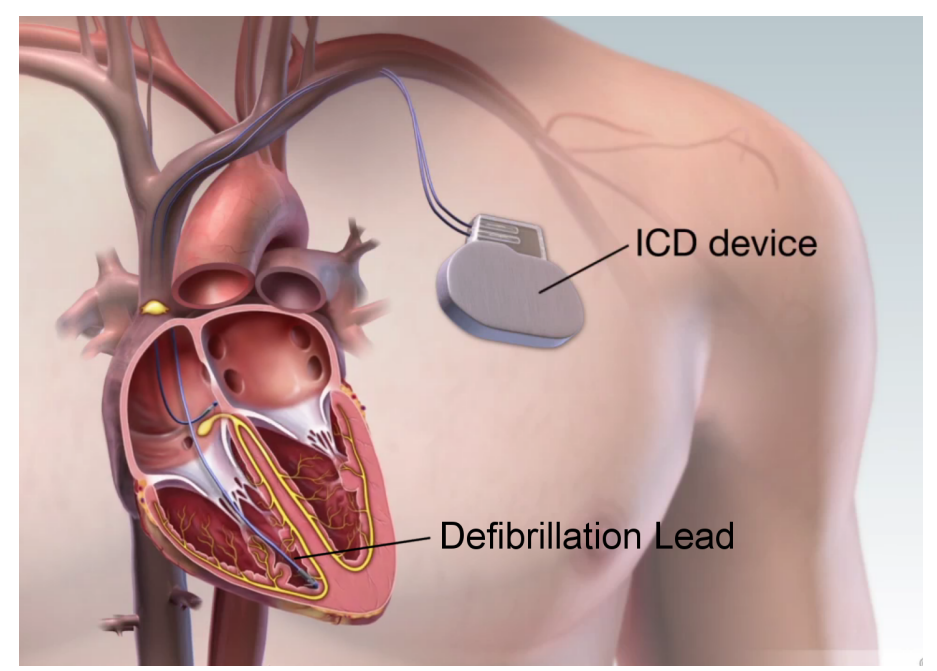

Figure 1.6: An implantable cardioverter-defibrillator (ICD). The ICD device is implanted under the skin of the patient. Here, the electrodes which generate the electrical field (Fig. 1.4(c)) are the defibrillation lead (typically located inside the right ventricle) and the ICD device itself. Image taken from [16, Copyright Medmovie.

Studies indicate, that a combination of any three of the listed factors increases significantly the risk for a sudden cardiac arrest [15].

\subsubsection{Defibrillation of the Heart}

The conventional method to terminate ventricular fibrillation and restoring the sinus rhythm is to apply an electrical far field defibrillation shock to the heart (Fig. 1.4(c)).

This procedure can be performed by an external defibrillator (electrodes are located outside the body), or by a device implanted inside the body, an implantable cardioverter-defibrillator (ICD). ICDs are in particular used in patients with an increased risk for ventricular fibrillation [7]. The ICD itself serves as one electrode, whereas the second electrode is usually located inside the right ventricle (Fig. 1.6).

A successful defibrillation attempt terminates the chaotic wave dynamics inside the heart, and the electrical conduction system can re-initiate the sinus rhythm. Statistically, the success rate of a shock attempt is proportional to the electrical field strength (and thus to the energy). The detailed mechanism underlying this behavior will be discussed in detail in section 2.1.5. However, the clinical requirement for a success rate close to $100 \%$ is using the conventional techniques only accessible with huge field strengths (corresponding to around $200 \mathrm{~J}$ to $300 \mathrm{~J}$ ). The resulting currents which are induced by the electrical field are mainly responsible for the severe side-effects of this technique:

- The large electrical field strength causes extreme pain [17].

- Induced electrical currents additionally damage the cardiac cells, and in that way increase the risk for further arrhythmias [18, 19].

- Specifically, patients with an ICD suffer from posttraumatic stress symptoms [17, 20]. 
Although current approaches achieve a reasonable energy reduction by using a sequence of low-energy pulses as S. Luther and F. H. Fenton et al. [21] or a multiple-step protocol [22], significant improvements of theses techniques are still needed. As stated at the beginning of this chapter, reducing the severe side-effects provides the long term goal of this thesis.

\title{
1.3 Dynamical Systems and Nonlinear Dynamics
}

\author{
Using a term like nonlinear science is like referring to the bulk of zoology as the study of \\ non-elephant animals.
}

- Stanislaw Ulam

Apart from the medical and biological understanding, the dynamics of the heart can from a point of view of a physicist also be interpreted as a trajectory in the state space of the system. The mathematical field which describes the dynamics of these complex systems is called the dynamical systems theory. The embedding of the dynamics of the heart as a complex biological system into this mathematical framework is closely tied to the process of modeling. For example, a specific state of the heart has to be parametrized by information like the geometrical position, the shape or the structure of the heart, ion concentrations of the cells and other quantities in order to contain all relevant information 3 . This parametrization defines at the same time the state space, where each state is associated to specific values of the parameters. The subject of how to model a complex system will be discussed in detail in section 2.3

Additionally, for the description of the underlying processes (e.g. the spread of excitation waves in an excitable medium) the theory of nonlinear dynamics is required. The theories of dynamical systems and nonlinear dynamics provides many concepts and tools, which can be used to analyze and evaluate the complex dynamics of cardiac arrhythmias from an extended point of view. Using these mathematical techniques for the purpose of improving a medical therapy in the end, may sound ambitious but also may be rather odd. That is, why this approach can only be successful if there is a close connection to experimental results and an ongoing exchange of experiences with clinicians. Only then, this interdisciplinary strategy can be fruitful and provides both, a deeper insight into the fundamental dynamics of chaotic systems, and also valuable knowledge for the improvement of current defibrillation strategies and development of completely new therapies.

\footnotetext{
${ }^{3}$ A perfect description and simulation of a real heart and its dynamical processes is basically impossible in practice. The selection of the relevant quantities to describe the system is a key element of the process of modeling.
} 


\subsection{The Scope of this Thesis}

As discussed in the previous section, the goal of this thesis is to investigate the dynamics of the heart (and in particular the nature of cardiac arrhythmias) using concepts and perceptions from the dynamical systems theory. On the basis of the interdisciplinary interplay between mathematical approaches and interaction with experimental and clinical knowledge and results, two scientific objectives are addressed:

- Derive an enhanced understanding of the dynamics during episodes of ventricular fibrillation, including the development of concepts for the improvement of current defibrillation techniques and suggestions for completely new strategies which may find their way into the clinical application.

- Obtain novel insights into the fundamental dynamics of complex, nonlinear systems (thus excitable systems and beyond).

These objectives shall be achieved using numerical simulations, which are in this thesis the main tool to investigate specific research questions.

The thesis is structured as follows: In the second chapter, an extensive overview is given over the biological backgrounds of the dynamics of the heart (section 2.1) and the mathematical concepts and tools used in the studies (section 2.2). Since the conclusions of this thesis are mainly based on the results obtained from diverse numerical simulations, the role of simulations in the general context of scientific research and specifically in the field of complex biological systems is discussed in section 2.3 .

Furthermore, in section 2.4 the governing differential equations are derived, and the numerical implementation concerning all features of the simulations is presented.

The third chapter comprises four studies, whose objectives are mainly motivated from experimental observations and results. The first three of the four studies are based on manuscripts which are currently at different stages of the publication process in peer reviewed journals (published, submitted and under revision). Therefore, each study is accompanied by a paragraph which states the current status of the manuscript (at the submission date of this thesis) and the individual contributions of the authors.

Furthermore, each study is introduced and concluded by a short paragraph which illustrates the respective scientific objective of the study and interprets the results concerning the initial question at the end. Although the individual research questions are related and build on one another, each study can therefore also stand on its own, and the interested reader could therefore also jump to a specific study, with occasional excursions to chapter 2 for background information and details about the used methods and tools.

The scientific objectives of the studies presented in this thesis are formulated in the following:

- What is the mechanism of spontaneous termination of ventricular fibrillation? Investigating the transient nature of chaotic dynamics in excitable media of spatially extended systems (section 3.1 on page 68 ). 
- Is a prediction of the end of a chaotic episode possible? Characterizing the final phase of transient chaos (section 3.2 on page 76 ).

- What is the underlying mechanism for complexity fluctuations in the electrocardiogram during an episode of ventricular fibrillation? Correlation between fluctuations in the spatio-temporal dynamics using time series from multiple electrocardiogram electrodes (section 3.3 on page 102.

- What is the least possible interaction for the successful control and termination of ventricular fibrillation? Concepts for minimal energy defibrillation (section 3.4 on page 117).

In the last chapter, the results obtained in the studies are summarized and discussed (section 4.1 . 


\section{Chapter 2}

\section{Methods}

This chapter provides the scientific foundations for the studies presented in this thesis. First, the major governing mechanisms of the heart are described (section 2.1), followed by the embedding of the dynamics into the mathematical context (section 2.2). In the third section 2.3 , the role of numerical simulations (in particular in relation to experimental results) is discussed. Finally, in section 2.4 the governing equations are derived, the relevant features of the simulations are explained, and the numerical implementation of these features, including the used analyzing tools, is presented.

\subsection{Complexity of the Heart}

The basic anatomy and functionality of the heart was already described in section 1.1 . However, for the implementation of sophisticated simulations of the dynamics of cardiac tissue a broad understanding of the underlying biochemical processes (up to a reasonable level of detail) and more details about diverse components of the cardiac substrate are required. These aspects will be revisited in section 2.4.2, where the modeling of the governing processes which are relevant for this thesis will be described. From a medical point of view, as well as under biophysical and biochemical considerations, the heart is a highly complex organ, exhibiting various mechanisms and processes on different length and time scales, which in total provide the proper pumping function. In this section, we describe and explain the most prevalent and governing processes and features that play a role for the dynamics of cardiac tissue. In doing so, we systematically go from a small scale (the dynamics of one cell) to larger scales (cell coupling, propagation of the electrical signal) and end up with explanations about the measurement of cardiac functionality (electrocardiogram) and the principles of the underlying processes during defibrillation (virtual electrodes, defibrillation threshold).

\subsubsection{Single Cell Dynamics - Cardiac Tissue as an Excitable Medium}

In this section we want to understand how the membrane potential of a cell (more precisely the voltage between the inside and the outside of a cell) is composed and what happens during an action potential on a cellular level. The electrical potential inside and outside of 
(a)

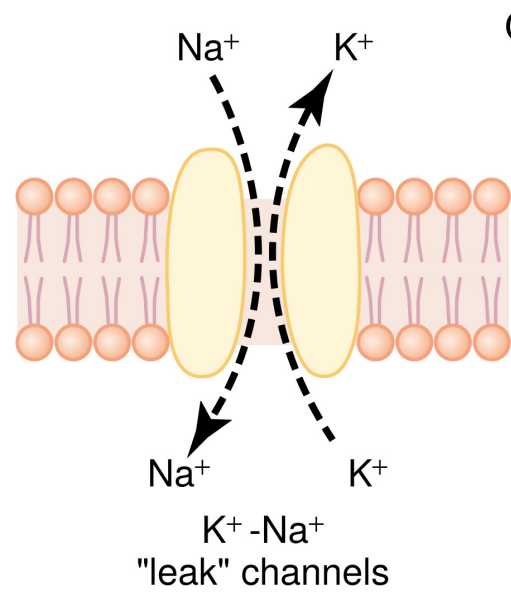

(b)

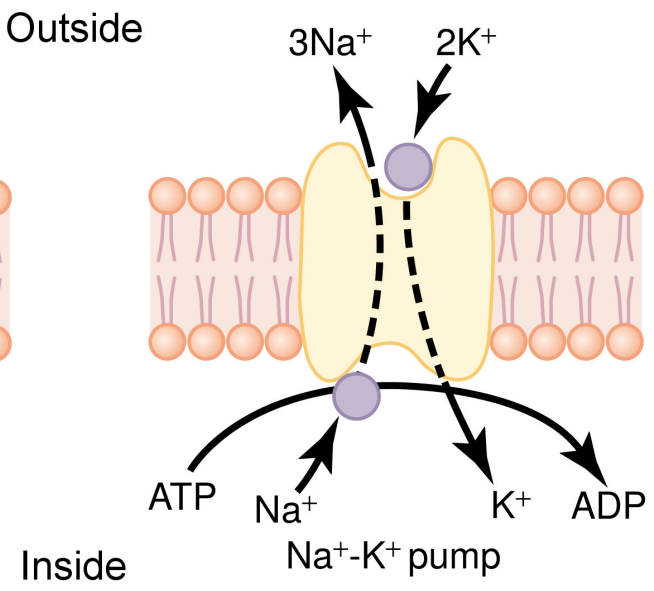

Figure 2.1: The ion channels which are mainly responsible for the resting potential $V_{\mathrm{m}}^{\text {rest }}$. In (a) the leak channels are depicted, which allow a steady transmembrane current of sodium and potassium channels. In addition, the $\mathrm{Na}^{+}-\mathrm{K}^{+}$pump provides for an exchange of three sodium ions from the inside to the outside, and at the same time a propagation of two potassium ions from the outside to the inside of the cell. This figure was published in [24, Copyright Elsevier (2018).

a cardiac cell is determined by ion concentrations of specific ions (e.g. sodium $\left(\mathrm{Na}^{+}\right)$, potassium $\left(\mathrm{K}^{+}\right)$, chloride $\left(\mathrm{Cl}^{-}\right)$or calcium $\left.\left(\mathrm{Ca}^{2+}\right)\right)$. The membrane, which separates the inside from the outside of the cell, is permeable for these ions under the consideration of different mechanisms. Goldman derived already in 1943 an expression for the membrane potential $V_{\mathrm{m}}$ depending on ion concentrations $\left[23\right.$. (denoted by square brackets $[x]_{\mathrm{i} / \mathrm{o}}$ for concentrations inside and outside the cell, respectively) and specific ion permeabilities (denoted by $P_{x}$ ), called the Goldman equation:

$$
V_{\mathrm{m}}=\frac{R T}{F} \cdot \frac{P_{\mathrm{Na}} \cdot\left[\mathrm{Na}^{+}\right]_{\mathrm{o}}+P_{\mathrm{K}} \cdot\left[\mathrm{K}^{+}\right]_{\mathrm{o}}+P_{\mathrm{Cl}} \cdot\left[\mathrm{Cl}^{-}\right]_{\mathrm{i}}}{P_{\mathrm{Na}} \cdot\left[\mathrm{Na}^{+}\right]_{\mathrm{i}}+P_{\mathrm{K}} \cdot\left[\mathrm{K}^{+}\right]_{\mathrm{i}}+P_{\mathrm{Cl}} \cdot\left[\mathrm{Cl}^{-}\right]_{\mathrm{o}}}
$$

where $R$ is the ideal gas constant, $T$ is the temperature and $F$ is Faraday's constant.

\section{Resting Potential}

The resting membrane potential is mainly determined by the sodium and potassium concentrations, other ions and proteins do only play a minor role here. The actual resting potential is primarily composed by two effects: leak channels of the cell allow the diffusion of sodium and potassium due to a non-zero concentration gradient of the ions (Fig. 2.1(a)). In addition, the sodium-potassium pump, also called $\mathrm{Na}^{+} / \mathrm{K}^{+}$-ATPase is an enzyme which pumps three sodium ions from the inside to the outside and at the same time two potassium ions from the outside to the inside of the cell (Fig. 2.1(b)). These two effects combined 
provide a resting potential of about $V_{\mathrm{m}}^{\text {rest }} \approx-90 \mathrm{mV} ! 1$

\section{Action Potential}

When an excitation wave travels through the tissue (which subsequently triggers the mechanical contraction (see section 2.1.3 on page 20 ), the local membrane potential $V_{\mathrm{m}}$ differs from the resting potential $V_{\mathrm{m}}^{\text {rest }}$ and one can observe an action potential (Fig. 2.2(a)), which is characterized by a rapid depolarization of the membrane potential (ii) from the resting potential $V_{\mathrm{m}}^{\text {rest }} \approx-90 \mathrm{mV}$ (i) to approximately $20 \mathrm{mV}$ (upstroke), followed by a relatively stable plateau, which determines the length of the action potential (iii). Subsequently, the membrane potential repolarizes (iv) back to the initial resting potential (v). The whole dynamics during this process is determined by the sophisticated behavior of voltage gated ion channels. By going step by step through the stages (i)-(v) in Fig. 2.2, we examine the respective underlying dynamics in each part.

Initially, the action potential is in the resting state ((i) in Fig. 2.2). The action potential is then triggered by a slight increase of the membrane potential $V_{\mathrm{m}}$ over the excitation threshold of about $V_{\mathrm{m}}^{\mathrm{th}} \approx-70 \mathrm{mV}$ (vertical orange line in Fig. 2.2(a)) by nearby cells (details about the propagation of the electrical signal are given in section 2.1 .2 on page 17). This causes the opening of specific voltage gated sodium channels.

Figure 2.3 sketches the dynamics of these ion channels for sodium (a) and potassium (b). The voltage gated sodium channels have two gates, an activation gate and an inactivation gate. During the resting state, the activation gate is closed, whereas the inactivation gate is open. When the membrane potential exceeds the excitation threshold, the activation gate opens and sodium ions pour (due to a concentration gradient) from the outside to the inside (sketched in Fig. 2.2(b), by definition a current from the outside to the inside is positive, the other way negative). This abrupt change in the ion concentrations leads to the upstroke of the membrane potential (phase (ii) in Fig. 2.2(a)). The inactivation gate is also triggered by the initial increase of the membrane potential, but it closes a few 10000ths of a second later than the opening of the activation gate. This mechanism leads to a temporal limitation of the sodium current. In contrast to the fast reaction of the sodium channels to a voltage change (therefore also denoted as "fast" channels), the voltage gated potassium channels (Fig. 2.3(b)) open a bit later, approximately at the same time as the sodium channels close. Potassium ions can then propagate from the inside to the outside of the cell (sketched in Fig. 2.2(b), the negative sign is due to the definition of the direction of the flow). This effect alone would cause a continuous decrease of the membrane potential, which can also be observed in many other types of cells, e.g. in nerve fibers. However, in cardiac muscle cells, the membrane potential remains relatively constant after the upstroke ((iii) in Fig. 2.2(a)), due to additional voltage gated calcium channels. These "slow" channels, allow calcium ions to propagate from the outside to the inside of the cell (Fig. 2.2(b)), and therefore counteract the decrease of the membrane potential caused by the propagation of potassium

\footnotetext{
${ }^{1}$ By definition, the membrane potential is counted from the inside (more negative) to the outside of the cell. This causes the negative sign of the resting potential.
} 


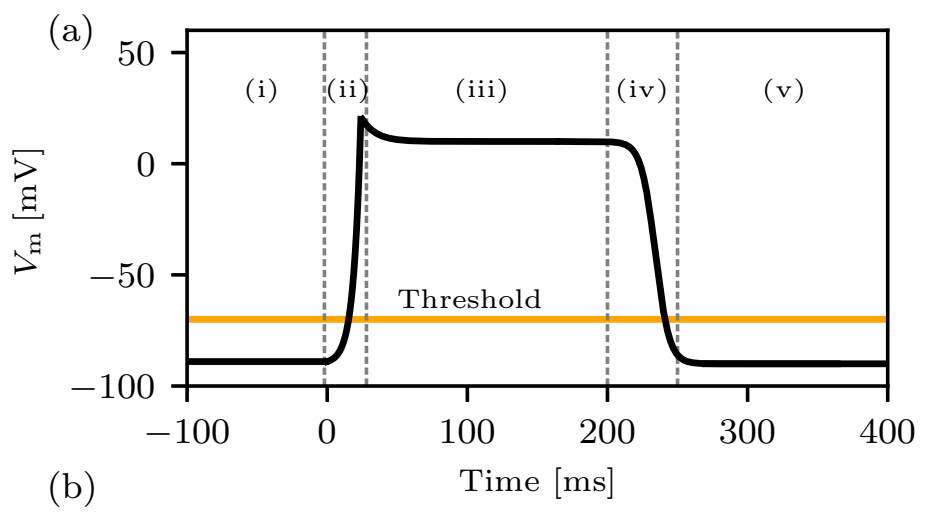

(b)

Time [ms]

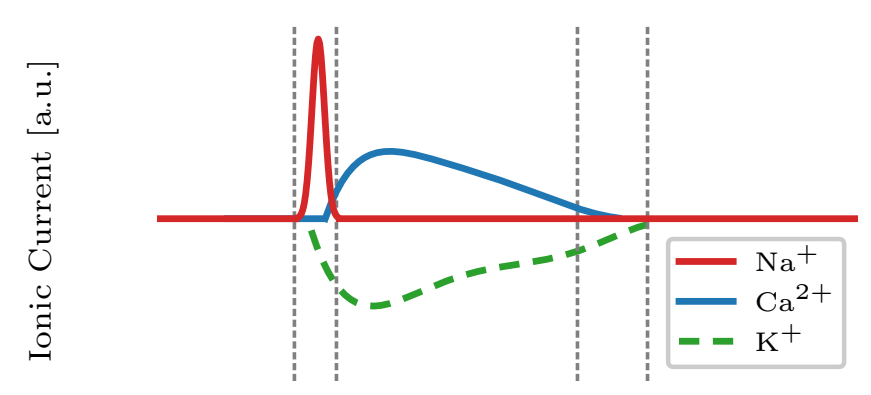

Figure 2.2: The action potential and its underlying mechanism. Subfigure (a) shows a generic action potential of cardiac muscle with a typical action potential duration of about $250 \mathrm{~ms}$ and a voltage threshold of about $V_{\mathrm{m}}^{\mathrm{th}} \approx-70 \mathrm{mV}$. The evolution of the membrane potential $V_{\mathrm{m}}$ can be subdivided into the initial resting state (i), the upstroke (ii), the following approximately constant plateau (iii), the repolarization (iv) and the return to the resting state (v). In (b), the transmembrane ionic currents are schematically depicted, which mainly determine the underlying dynamics of the action potential. The initial increase of $V_{\mathrm{m}}$ over the excitation threshold causes the voltage gated sodium channels to open for a short time (Fig. 2.3), resulting in the upstroke of $V_{\mathrm{m}}$ (ii). The "slow" potassium and calcium channels open later, and allow the propagation of calcium ions from the outside to the inside (blue curve) and potassium ions from the inside to the outside of the cell (green dashed curve). The direction of the flow is clarified by the negative sign of the potassium current. The two currents approximately annihilate each other (in terms of an effective change of $V_{\mathrm{m}}$ ) and determine the plateau of the action potential (iii). Eventually, the calcium channels close earlier than the potassium channels (iv), thus the potassium current predominates and causes the repolarization of the membrane potential back to the resting state (v). 


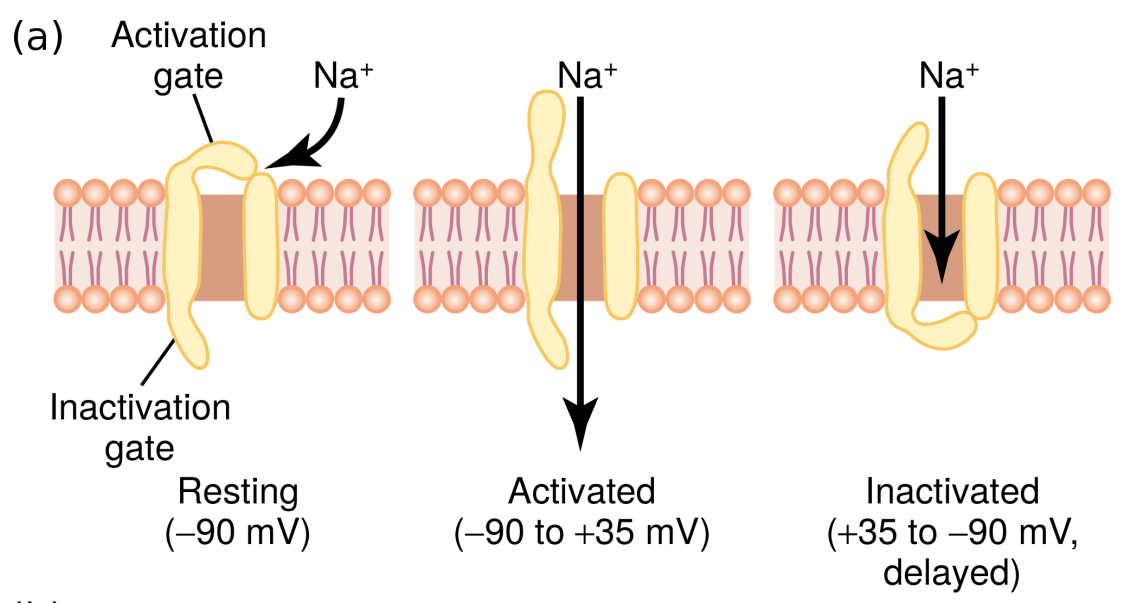

(b)

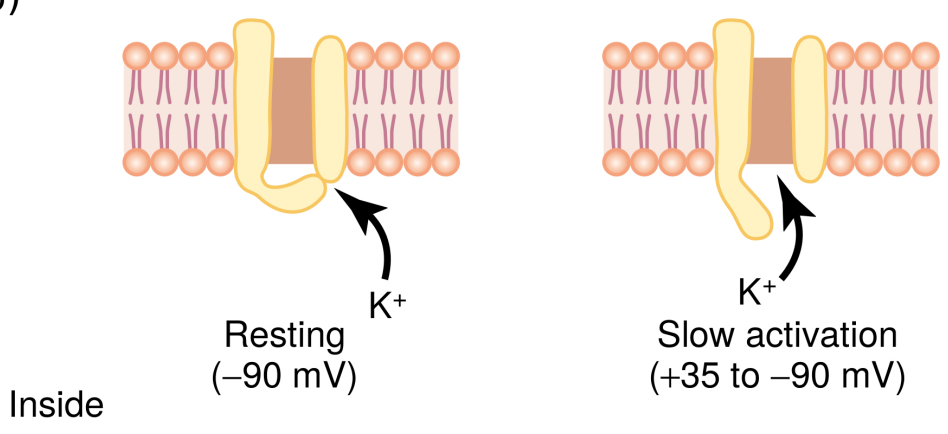

Figure 2.3: A basic description of the mechanism of voltage gated ion channels. In (a) three stages of voltage gated sodium channels are shown, respective to different states during an action potential. During the resting state, the activation gate is closed and the inactivation gate is open (left). When the membrane potential of the cell exceeds the excitation threshold, the activation gate opens and allows sodium ions to propagate (due to a concentration gradient) from the outside to the inside of the cell (middle). After less than a millisecond, the inactivation gate closes (right). The channel returns to the initial state (left) only, when the membrane voltage reaches the resting state again. The voltage gated potassium channels (b) behave similar, despite the fact that they possess only one gate. This gate opens and closes also triggered by the membrane potential, but this process happens much slower than the voltage gated sodium channels. This figure was published in [24, Copyright Elsevier (2018). 
ions. Finally, the calcium channels slowly close and the potassium channels predominate, leading to the repolarization of the membrane potential (iv) back to the resting state (v). The resulting action potential can be characterized by a certain action potential duration APD (Fig. 2.2(a)) of about APD $\approx 200 \mathrm{~ms}$ to $300 \mathrm{~ms}$. Due to the heterogeneous substrate of the heart, the exact lengths and durations and related details of the respective ion channel dynamics can vary from region to region (e.g. endocardium, epicardium, pacemaker cells). Another distinctive feature of the dynamics is the existence of a refractory period, an amount of time (subsequently to the upstroke of the action potential, and of comparable length as the action potential duration), where the cell cannot be excited again. The inactivation gates of the voltage gated sodium channels do not open again, if the membrane potential returns to (or almost to) the resting potential of approximately $-90 \mathrm{mV}$, even if a second signal arrives from another cell. This behavior stabilizes the proper dynamics of the heart and impedes disorganized contraction patterns. With the existence of a resting state, an excited state, and a refractory state, cardiac tissue can be recognized as an excitable medium. As a remark, the ion channel dynamics discussed here is mainly responsible for the action potential, but also other kinds of ion channel dynamics contributes to the final shape of the action potential (e.g. chloride ions or "fast" potassium channels [25]). The desired level of detail describing the actual dynamics is, however, in particular essential for the process of modeling (for the role of models in numerical simulations see section 2.3 on page 40 . 


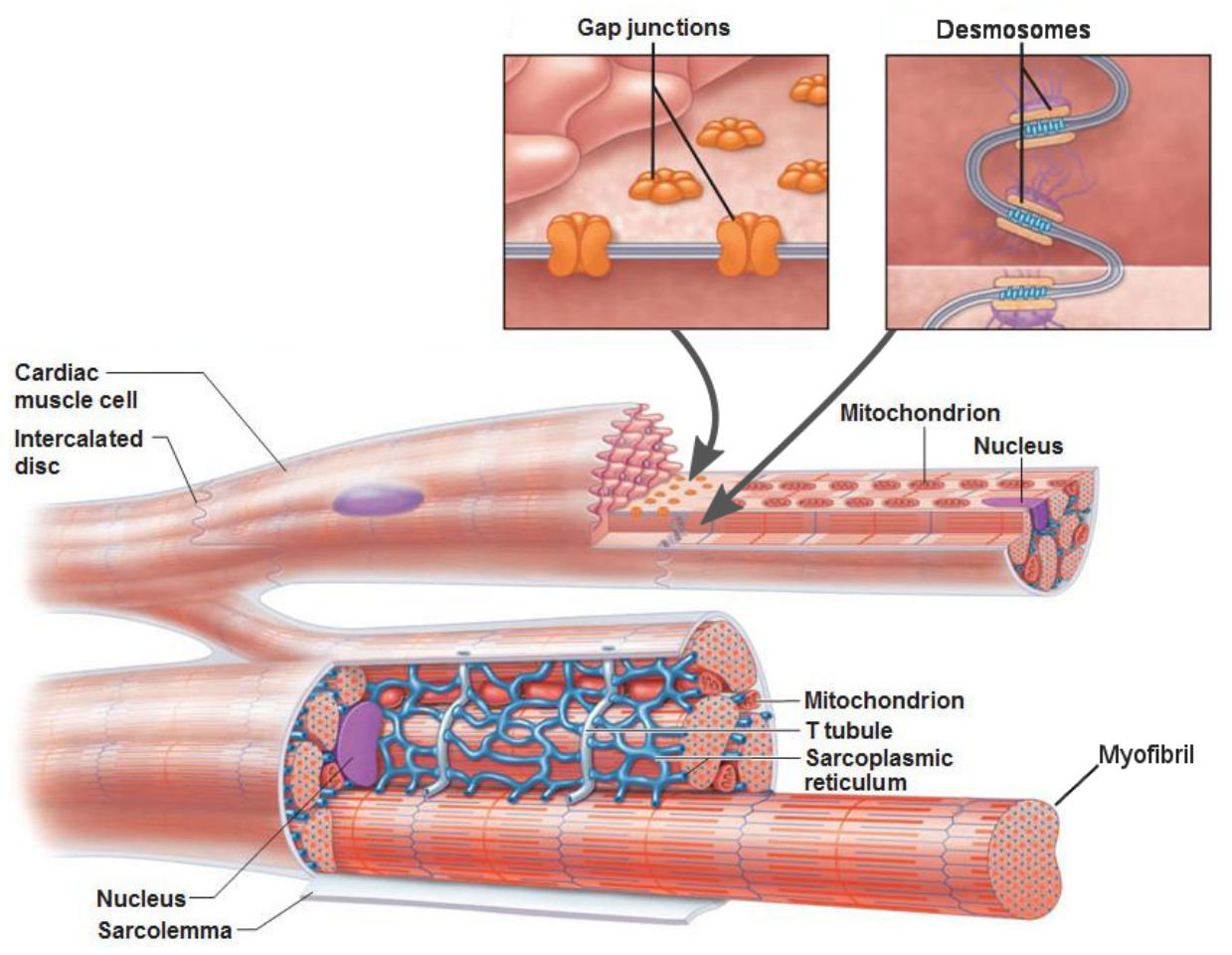

Figure 2.4: The anatomy of cardiac muscle fibers. The lower part of the figure shows a schematic drawing of cardiac muscle fibers and its constituents. Magnified sketches of the gap junctions (left plot of the upper part) and the desmosomes (right plot of the upper part) are depicted. Reprinted by permission of Pearson Education, Inc., New York, New York [1].

\subsubsection{Signal Propagation on Different Scales}

In the previous section we discussed how an action potential arises. Now we want to understand, how the electrical signal propagates through the tissue. For this purpose, we describe here the anatomical structure of cardiac muscle fibers.

\section{Cell to Cell Coupling}

The lower part of Fig. 2.4 shows a schematic picture of cardiac fibers, which are encased by the sarcolemma. The cardiac cells possess a nucleus and they are separated by intercalated discs (also shown in a photomicrograph of cardiac muscle in the upper left part). The intercalated discs consist of gap junctions, that actually allow the propagation of ions from one cell to a neighboring cell and desmosomes, which bind the cells together and ensure in this way the mechanical contraction of the whole muscle. Furthermore, the large mitochondria (around $25 \%$ to $35 \%$ of the volume in cardiac cells, whereas in comparison $\approx 2 \%$ in skeletal muscle) provide the adenosine triphosphate (ATP) supply of the cell, and make the cardiac cells resistant to fatigue. The myofibrils (the parts of the cell which provide the actual mechanical contraction) constitute most of the remaining space of the cell. The electrical signal is propagating intracellular (from cell to cell) as well as extracellular along the sarcolemma. The t-tubules are basically (transverse) invaginations of the sarcolemma, and 
(a)

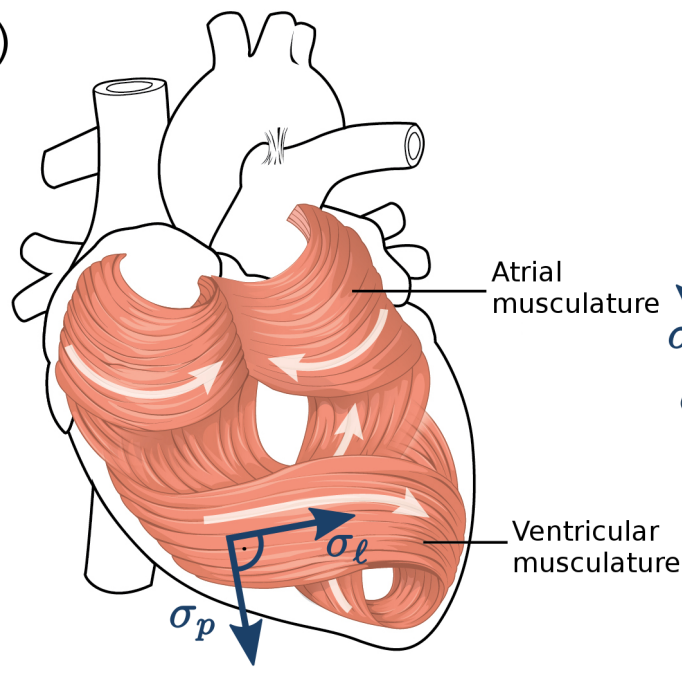

(b)

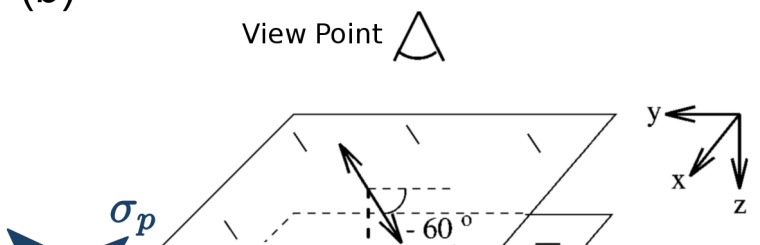

Figure 2.5: The arrangement of cardiac fibers in the heart. The subfigure (a) depicts a sketch of the fiber alignment in atria and ventricular muscle. Blue arrows indicate the defined direction, corresponding to the conductivities $\sigma_{\ell}$ and $\sigma_{p}$ for one exemplary point of the tissue. Reprinted by permission of Pearson Education, Inc., New York, New York [1]. The rotation of fiber sheets from the epicardium to the endocardium is shown in (b). As in (a), blue arrows indicate the conductivities which correspond to the longitudinal direction along the fibers $\left(\sigma_{\ell}\right)$, the perpendicular direction, orthogonal to the fiber direction but within the fiber sheet $\left(\sigma_{p}\right)$ and the transverse direction, orthogonal to the first two directions, with a transmural direction $\left(\sigma_{t}\right)$. Reprinted from [26, with the permission of AIP Publishing.

allow the quick propagation of the extracellular signal into the inner part of the cell. Here, the electrical signal can enter the intracellular domain by a large number of ion channels. The last constituent of the cardiac cell depicted here is the sarcoplasmic reticulum, basically a huge storage of calcium, which is essential for the mechanical contraction of the cell (see section 2.1 .3 on page 20 .

\section{Arrangement of Cardiac Fibers}

After discussing how an action potential propagates from cell to cell, we now want to clarify how the electrical signal spreads on a global scale, thus in the whole organ. In particular, the question arises: does the propagation of the electrical signal occur homogeneous and/or isotropic? In fact, cardiac muscle fibers are arranged in such a way, that the pumping function is optimized in a highly efficient way.

In Fig. 2.5(a) the direction of the fibers is sketched. The sophisticated structure and arrangement causes a screw-like contraction, which pushes the blood out of the heart. In detail, (tube-like) cardiac cells are arranged in layers or sheets, parallel to the surface of the heart. Three distinct directions can be defined at every point of the tissue (also marked in Fig. 2.5(a) and (b)): the direction within the layer and along the fibers (longitudinal direction), the direction perpendicular to the fiber, but within the layer (perpendicular direction) and the direction which is orthogonal to the first two directions, and thus transverse to the layer (transverse direction). Since the sheets of tissue are aligned parallel to the surface 


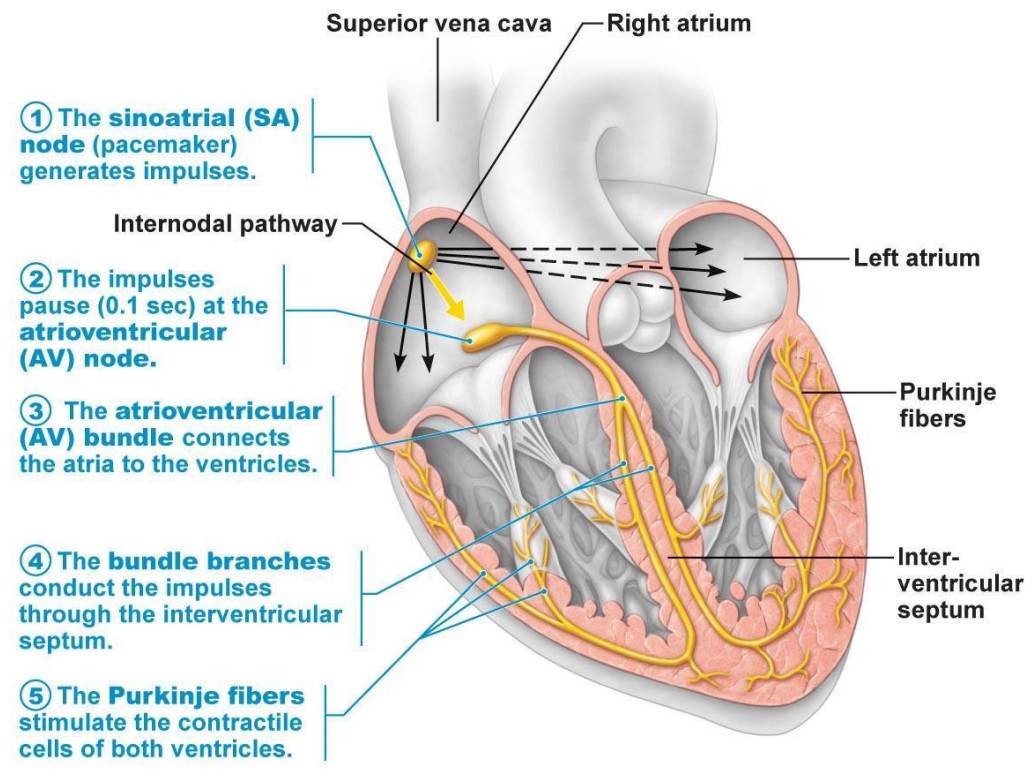

Figure 2.6: The conduction system of the heart. The pathway of the electrical signal is initiated by the sinoatrial node (1). It propagates through the atria (causing their contraction) and along the internodal pathway to the atrioventricular node (2). After a delay of about $0.1 \mathrm{~s}$, the signal continues to travel in the atrioventricular bundle (3), which splits up into the left and right bundle branches (4). At the end, the electrical signal propagates through the Purkinje fibers (5), where it connects to the cardiac cells and initiates the contraction of the ventricles. Reprinted by permission of Pearson Education, Inc., New York, New York [1].

of the heart (except e.g. in the septum), the transverse direction gives in general also the transmural direction. Furthermore, neighboring layers of tissue are rotated against each other in such a way, that the fiber direction (longitudinal direction) rotates monotonously $120^{\circ}$ from the outside (epicardium) to the inside of the heart (endocardium) (Fig. 2.5(b)). Due to the anatomical heterogeneity of the tissue, the electrical conductivities in these three directions $\left(\sigma_{\ell}, \sigma_{p}\right.$ and $\left.\sigma_{t}\right)$ can differ significantly in their magnitude from each other and additionally can depend on the region of the heart, too.

\section{Electrical Conduction System of the Heart}

The frequency of contractions of the heart muscle is determined by the autonomic nervous system. In particular, the sympathetic and the parasympathetic nervous system may increase or decrease the heart rate, respectively, depending on the actual condition and need of the whole body. The actual process of a mechanical contraction, however, is triggered by the heart itself.

The heart initiates intrinsically the contraction from the sinoatrial (SA) node (an autonomous pacemaker, see Fig. 2.6) and the electrical signal is then distributed inside the heart muscle, using a sophisticated electrical conduction system. From the SA node, the electrical activity spreads throughout the atria, causing a contraction here and travels along the internodal pathway to the atrioventricular $\mathrm{AV}$ node. The propagation of the electrical 


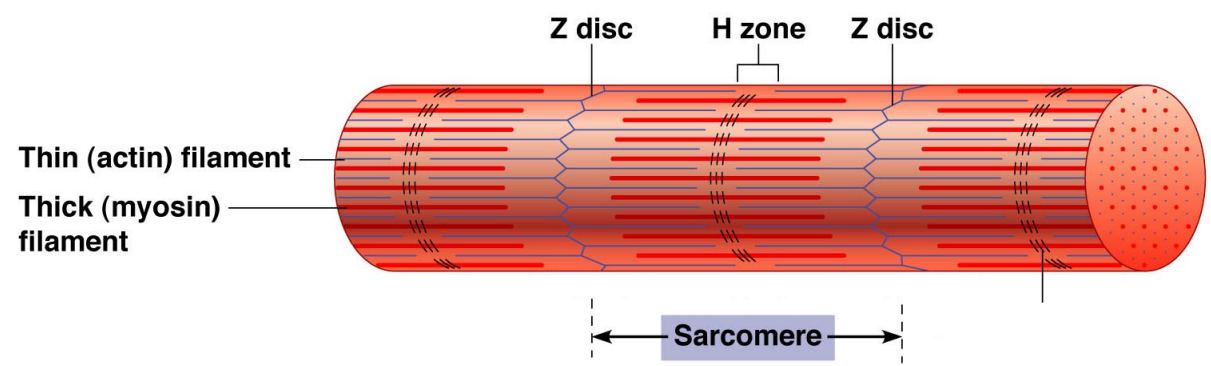

Figure 2.7: The structure of a moyofibril. Myofibrils are composed of sarcomeres, which are separated by $\mathrm{Z}$ discs. The sarcomeres consist mainly of thin (actin) filaments, and thick (myosin) filaments. In the case of a contraction of the muscle, the actin filaments are moving (with the $\mathrm{Z}$ discs) towards the myosin filaments (thus in the direction of the $\mathrm{H}$ zone). Reprinted by permission of Pearson Education, Inc., New York, New York [1].

signal is delayed here for around $0.1 \mathrm{~s}$, which allows the atria to complete the contraction. The atrioventricular node is (in the case of a healthy heart) the only electrical connection between the atria and the ventricles. From here, the impulse travels along the atrioventricular bundle inside the septum of the heart, and splitting then up into the left and right bundle branches, heading to the apex of the heart. The Purkinje fibers represent the final part of the conduction system. Only here, the electrical signal connects to the cardiac muscle and induces the mechanical contraction of the ventricles.

\subsubsection{Mechanical Contraction}

The main task of the heart is its mechanical contraction which pumps the blood through the body. The electrical signal initiates the actual process of contraction 2 which occurs inside the myofibrils (Fig. 2.4). The myofibrils can be divided into sarcomeres, single contracting units, which are separated by $Z$ discs (Fig. 2.7).

The sarcomeres are a compound of thin and thick filaments, where the thin filaments mainly consist of actin, coiled by nebulin and the thick filaments consist of myosin, which are held in place by titin filaments. A mechanical contraction is triggered by an electrical signal, which initiates a huge calcium release by the sarcoplasmic reticulum (see section 2.1.2). These calcium ions diffuse between the actin and myosin filaments. This leads to a force, that pulls the actin filaments towards the middle of the sarcomere ( $H$ zone), until they overlap with the myosin filaments. The result is a contraction of the sarcomere, and since the cardiac cells are electrically connected, the whole heart muscle contracts in an organized way.

\footnotetext{
${ }^{2}$ As described in section 1.1 we concentrate in this thesis on the electrical excitation patterns, and assume that the mechanical contraction is unidirectionally determined by the electrical signal.
} 


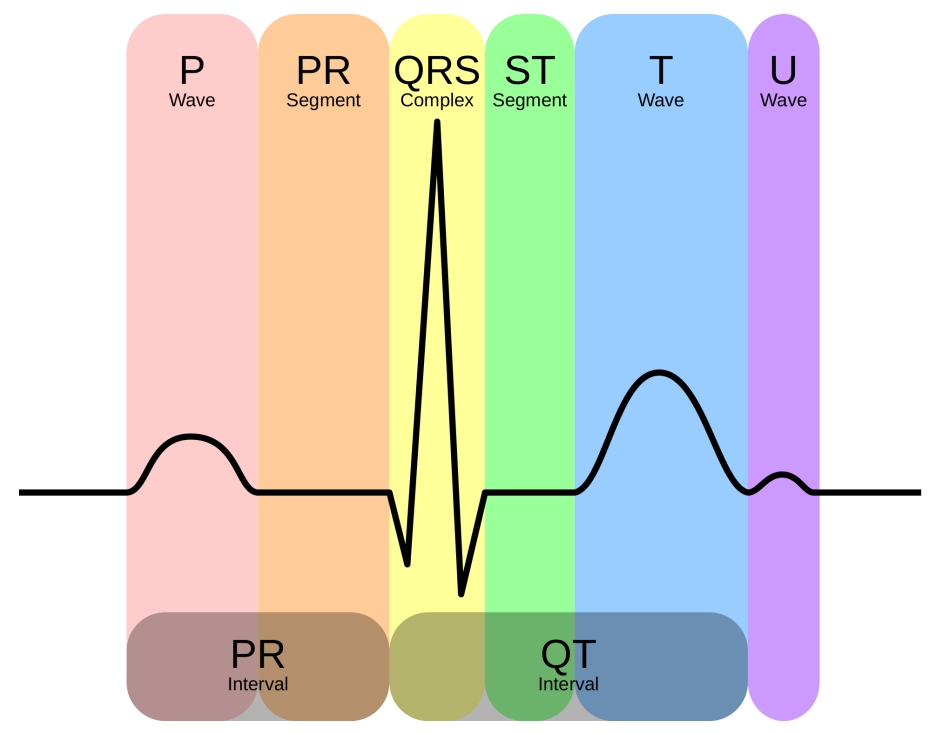

Figure 2.8: Schematic representation of a usual ECG during sinus rhythm. Different stages of the mechanical contraction of the heart, can be associated to subsequent sections of the ECG ( $P$ wave, $P R$ segment, $Q R S$ complex, $S T$ segment, $T$ wave and the $U$ wave). Image reprinted from Wikimedia Commons [27].

\subsubsection{The Electrocardiogram}

The rhythmical contraction of the heart can also be measured from outside the body (noninvasively) using electrocardiogram (ECG) electrodes. This technique provides valuable insight into the underlying dynamics of the heart. In particular, the ECG electrodes do not measure directly the mechanical motion of the heart, but rather the electrical excitation patterns. During the usual sinus rhythm, the electrocardiogram provides a characteristic signal (schematic representation in Fig. 2.8), where each part can be associated to different stages of the contraction.

The mechanical contraction starts with the depolarization of the atria, which can be associated with the $P$ wave in the electrocardiogram, followed by the $P R$ segment (propagation of the signal through the atrioventricular bundle) which lasts until the ECG drops, defining the start of the QRS complex (for comparison to the electrical conduction system, see section 2.1.2 on page 17). After the signal has passed the atrioventricular bundle, the interventricular septum depolarizes, inducing a drop of the ECG signal ( $Q$ wave), followed by the depolarization of the remaining ventricular muscle ( $R$ wave). Afterwards, the repolarization of the ventricles is reflected in the $S T$ segment, the $T$ wave and the $U$ wave. Since the different parts of the ECG (at least partially) refer to distinct regions of the heart, physicians can deduce (possibly pathological) anatomical variations of the heart from alterations of the normal shape of the ECG. In the conventional way, the ECG is mainly used for analyses of the sinus rhythm, and related heart diseases. In the study of section 3.3 on page 102 we extend this concept, by introducing a multiple electrode measurement during ventricular fibrillation, and derive statements about the spatio-temporal state of the heart during the chaotic dynamics. 


\subsubsection{Defibrillation of the Heart}

Defibrillation describes a process that terminates cardiac arrhythmias (e.g. ventricular fibrillation), via the application of electrical high-energy far field shocks to the heart. This procedure, and in particular the significant side effects of current standard defibrillation technique provide the main motivation of this thesis. The purpose of a defibrillation attempt is, to terminate chaotic excitation patterns present in the heart, and to restore sinus rhythm. In the following, the underlying mechanism of the application of high-energy far field shocks, and their effect on cardiac tissue is discussed.

\section{Virtual Electrodes}

The concept of virtual electrodes is fundamental for the understanding of the underlying mechanisms of defibrillation [28, 29]: The cardiac muscle is not a homogeneous substrate but heterogeneities of various size and structure are present in the tissue. Essential for the mechanism here are changes in the conductivities which emerge for example due to blood vessels, or discontinuities between bundles and sheets of fibers [30], but also the inner and outer boundary of the muscle (endocardium and epicardium, respectively) are crucial from this point of view. When an electrical field is applied for a certain amount of time ("far field shock"), the ions present in the tissue experience the Lorentz force. These charged particles are slightly shifted due to the force and accumulate at heterogeneities (or more precisely, at regions of a varying conductivity). Since the conductivities are different in the intraand extracellular space, the membrane potential exceeds the excitation threshold at specific locations, which then triggers an action potential there. In this way, heterogeneities can be denoted as "virtual electrodes", since excitation waves induced by an external electrical field are created here. The process of creating an excitation wave (using an external electrical field) at a certain heterogeneity is also termed "recruiting" the heterogeneity.

Quantitatively, the actual field strength plays an important role for the defibrillation process. Studies indicate, that a certain field strength of the external electrical field, can only "recruit" heterogeneities down to a specific size or shape [28, 29, 31]. That means, with a lower field strength, excitation waves are created only at some heterogeneities. For exam-

ple, P. Bittihn et al. showed [31], that the curvature of the heterogeneity is essential for the recruiting process (Fig. 2.9). Boundaries with a negative curvature (convex) can be excited with significantly lower field strengths, whereas heterogeneities with a flat or positive curvature (concave), are recruited only with high field strengths. That means, with an increase of the field strength, more and more heterogeneities can be recruited, and thus the regions of the heart where excitation waves are created grows monotonously. 
(a)
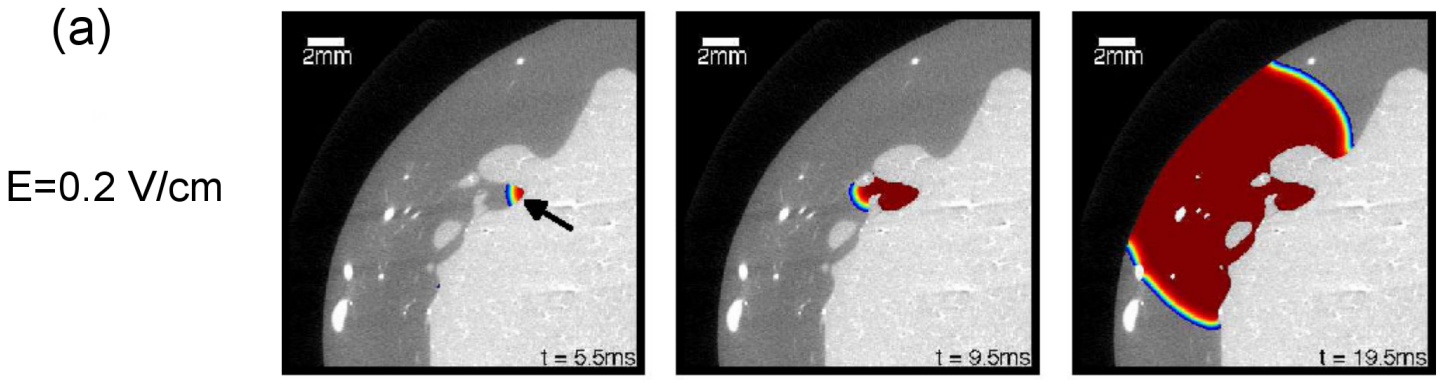

(b)
$=0.4 \mathrm{~V} / \mathrm{cm}$
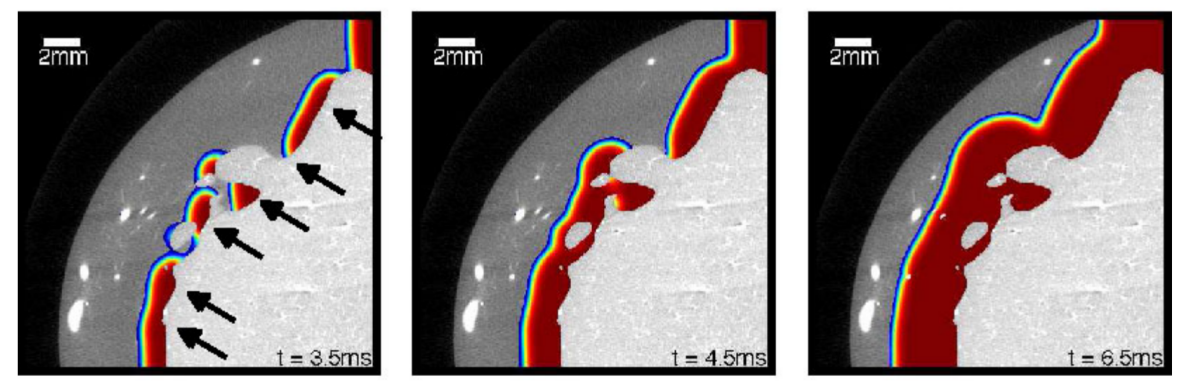

(c)
$E=1.0 \mathrm{~V} / \mathrm{cm}$
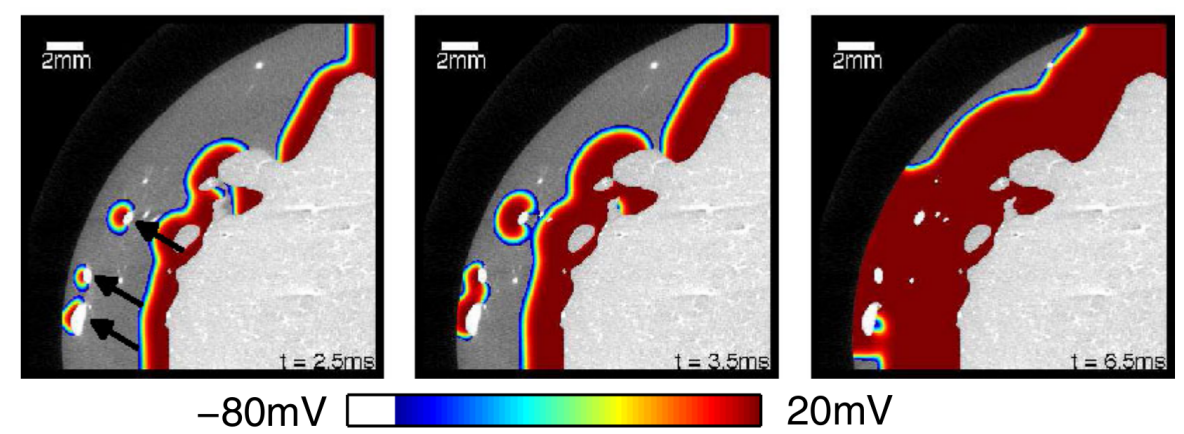

Figure 2.9: Recruiting of excitation sites in cardiac tissue dependent on the field strength. Results of numerical simulations using the Fenton-Karma model, based on a micro-CT (computed tomography) scan of the left ventricle of a rabbit heart. Excitation waves are depicted, which were created by the application of an electrical field for $5 \mathrm{~ms}$ with a varying field strength E. The plots show snapshots of the excitation patterns at different points in time (from left to right) and with different field strengths ((a) $0.2 \mathrm{~V} / \mathrm{cm}$, (b) $0.4 \mathrm{~V} / \mathrm{cm}$, and (c) $1.0 \mathrm{~V} / \mathrm{cm}$, respectively). Reprinted from [31] under the terms of the Creative Commons Attribution 3.0 License. 


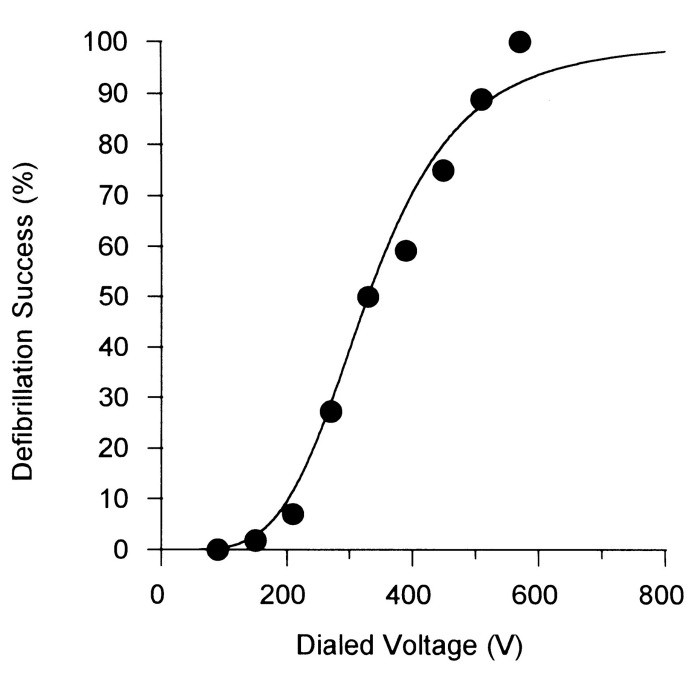

Figure 2.10: The dose response curve. In a defibrillation study [32] with 23 Langendorffperfused rabbit hearts, the defibrillation success rate (dose response curve) was determined statistically in 273 shock episodes. The sigmoid-like behavior of the success rate reflects the underlying mechanism of the defibrillation, which is based on the (energy dependent) recruitment of virtual electrodes. Reprinted from [32, with permission from Wolters Kluwer Health, Inc. .

\section{The Dose Response Curve}

In this picture, the standard defibrillation procedure (one high-energy shock) uses such a huge field strength, that it excites basically the whole tissue at once. In this way, the excitation waves which perpetuate the current arrhythmia cannot further propagate, the whole tissue is excited and decays then globally in the refractory state. Afterwards, the conduction system can restart the sinus rhythm and the ordered contraction of the heart. Using such a high energy/field strength ensures (in most cases) the termination of the cardiac arrhythmia, but comes along with significant side effects (discussed in section 1.2 on page 4), which are often related to the resulting electrical currents.

In comparison, using lower field strengths decreases the number of recruited heterogeneities. That means, the electrical field does not excite the whole tissue, but only parts of it. However, with a certain probability, the induced excitation waves can still terminate the present dynamics of the arrhythmia. Naturally, this probability is (in general) proportional to the field strength (and thus the number of excitation sites) [21]. The dose response curve (DRC), which gives the statistically determined success rate of a defibrillation shock dependent on the electrical field strength, reflects this behavior (Fig. 2.10).

Apparently, using a lower field strength/energy for defibrillation significantly reduces the probability for the termination of the arrhythmia (which is not acceptable regarding a clinical application). That is, why low energy defibrillation protocols (e.g. [21]) need to adjust parameters like the number, frequency or timing of the shock(s) in order to guarantee a reasonably high success rate. 


\subsection{Mathematical Embedding}

After the discussion of the governing principles and mechanisms which play a role in the dynamics of the heart, this chapter provides the mathematical framework of the simulation studies of this thesis. The dynamics of the heart is set into the context of the theory of dynamical systems, and important concepts and tools are described which will be used to interpret the numerical results. In particular, the phenomenon of chaotic transients is discussed in detail in section 2.2.3, since it plays a major role in this thesis.

\subsubsection{The Heart as a Dynamical System}

The embedding of the dynamics of cardiac arrhythmias into the theory of dynamical systems provides the scientific foundation for the numerical simulations and their subsequent interpretation presented in the studies of this thesis. The investigation of cardiac dynamics from this rather mathematical point of view allows the use of various methods and tools which are commonly used in nonlinear dynamics, and in particular enables the transfer of an episode of cardiac arrhythmia to a trajectory in state space. For this purpose the relevant quantities and concepts of the theory of dynamical systems which are needed for the discussion of the obtained results of this thesis are introduced in this section.

Mathematically, a dynamical system is defined by a tuple $(T, X, \Phi)$, where $T$ is a set representing time, $X$ is the state space and $\Phi$ is a function which maps an element from $T$ and $X$ to $X$, thus evolves a state through the state space: $\Phi: T \times X \rightarrow X$. For the sake of convenience, we will discuss in the following the main principles using the specific example of cardiac dynamics.

At the beginning, the state of the heart at time $t$ shall be described by a vector $\mathbf{x}(t)$, which includes all relevant information necessary to describe the state of the heart. Actually, the question which kind of information is sufficient for a full characterization of the status of the heart is essential and highly related to the scientific objectives of the respective study. It is clear, that in practice a state vector of finite length can only describe a small amount of the full information of the entire organ. This crucial process of modeling is further discussed in section 2.3 on page 40 . Here, we can assume that for a specific study $\mathbf{x}(t)$ contains the membrane potential and the ion concentrations of specific ions $3^{3}$ The temporal evolution of the state vector in time is then described by the function $\mathbf{f}(\mathbf{x}(t), t)$ :

$$
\frac{\partial \mathbf{x}(t)}{\partial t}=\mathbf{f}(\mathbf{x}(t), t)
$$

Equation 2.2 can be solved (analytically or numerically) using an initial condition $\mathbf{x}_{0}=$ $\mathbf{x}\left(t_{0}\right)$, which yields the trajectory $\gamma_{\mathbf{x}_{0}}$ concerning the initial condition. Thus, $\gamma_{\mathbf{x}_{0}}$ is a set of state vectors which are passed during the evolution of the initial condition through the state space $X$ (Fig. 2.11).

\footnotetext{
${ }^{3}$ Further information could include e.g. the mechanical contraction of the heart, thus the spatial position and shape of cells.
} 


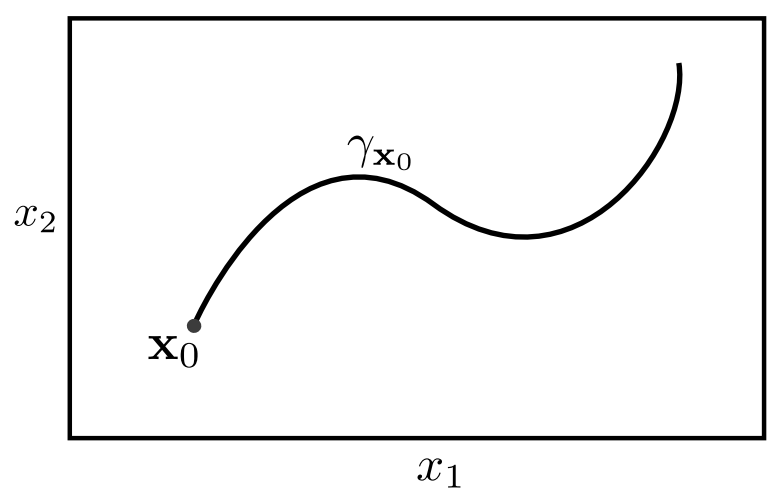

Figure 2.11: A sketch of a trajectory in state space. The (two-dimensional) state space is characterized by the parameters $x_{1}$ and $x_{2}$. The trajectory $\gamma_{\mathbf{x}_{0}}$ corresponding to the initial condition $\mathbf{x}_{0}$ depicts the temporal evolution of the state vector through the state space.

The aim of this thesis is to study these trajectories in state space, specifically the trajectories which correspond to the dynamics of episodes of cardiac arrhythmias. In particular, we are interested in possible structures or patterns of the state space. In a first step, we want to investigate whether topological structures which are commonly used to describe the state space of a system (e.g. attractors, repellers, saddles) can also be found in cardiac dynamics. After the identification of those objects one can then discuss how the results need to be interpreted concerning cardiac dynamics, in particular the implications for cardiac arrhythmias, and how the gained knowledge can be exploited for possible applications.

As already mentioned, for this purpose topological structures of the state space which are commonly found in nonlinear systems are described in the next section.

\subsubsection{Structures of the State Space}

In the following, typical topological objects of the state space are discussed. In many cases, the dynamics of a system is determined by the coexistence of more than one of these objects.

\section{Attractor}

An attractor of a system is a subset of the state space $X$, which tends to have an attracting effect on trajectories. The subset $A$ of the state space is called an attractor of the system if it fulfills three properties:

- When $\mathbf{x}\left(t_{1}\right)$ is an element of $A$, then it will remain in $A \forall t$ ( $A$ is an invariant set).

- For each attractor $A$ there exists the basin of attraction, a neighborhood of $\mathrm{A}$, called $\mathrm{B}(\mathrm{A})$. The basin is defined by the set of all points, which will asymptotically converge to the attractor in the limit $t \rightarrow \infty$.

- No non-empty subset of A exists, which fulfills the first two properties. 


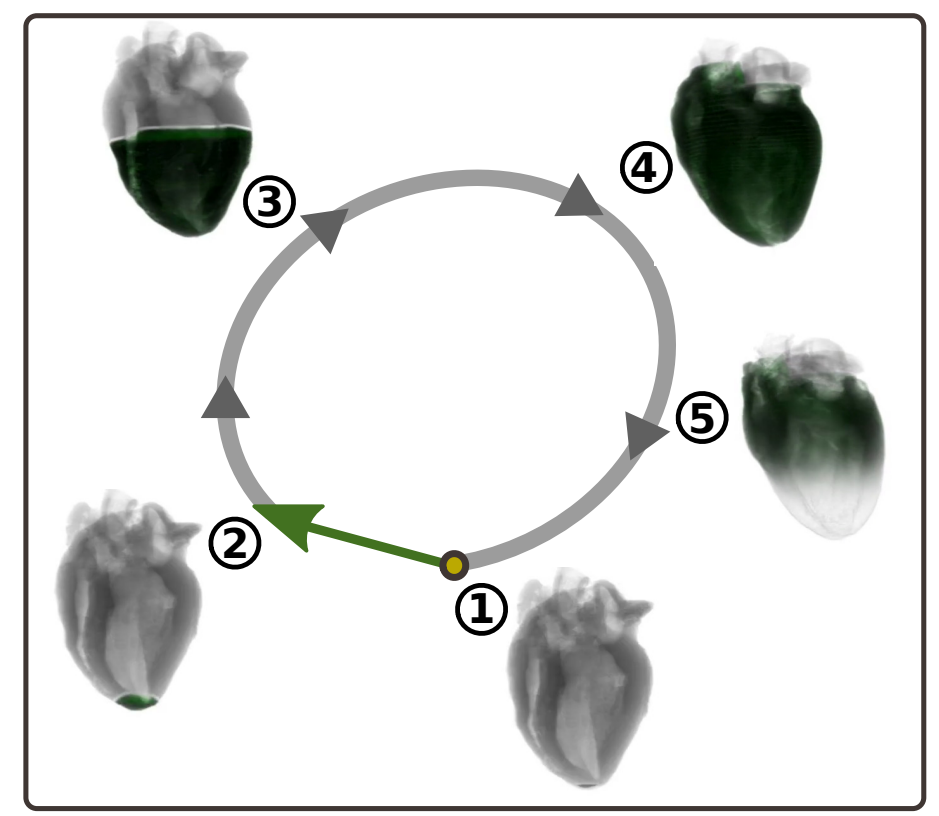

Figure 2.12: The sinus rhythm in state space. The rhythmical contraction of the heart (sinus rhythm) can be interpreted as a trajectory in state space and is here shown schematically. The resting state (fixed point), where the membrane potential everywhere in the heart is equal to the resting potential (section 2.1.1 on page 11) is in the figure marked by (1). The beginning of the contraction cycle initiated by an excitation of the av node can be interpreted as a perturbation from the resting state (2). As the plane wave propagates through the tissue, the state of the system travels through the state space ((3), (4) and (5)), until the plane wave has passed through the whole heart, and the system returns again to the resting state (1). (Simulation by Sebastian Stein).

\section{Fixed Point}

A stable fixed point is a special case of an attractor, where the attractor itself is given by a single point in the state space. By definition, the fixed point maps to itself. In many cases, the stability of the fixed point, thus the robustness against infinitesimal perturbations, and its basin are of interest. Actually, the principle of the basin of a fixed point can be found in cardiac dynamics as a practical example, in particular during the cycle of a usual (sinus rhythm) contraction. Here, the resting state between two contractions, defined by the absence of electrical waves, can be interpreted as a stable fixed point (marked by (1) in Fig. 2.12.

The fixed point is stable against small local perturbations of the membrane potential, which do not exceed the excitation threshold. However, if the perturbation is big enough (green arrow in Fig. 2.12), an action potential is induced. The external stimulus (which starts the cycle) is initiated by the sinus node and is forwarded by the electrical conduction system (section 2.1.2 on page 17). While the electrical wave propagates through the tissue $((3),(4)$ and (5)) the state of the system forms a trajectory in the state space. Since the system returns to its fixed point (1), the state in state space which corresponds to the initial perturbation (2) is thus also part of the basin of the fixed point. 
As a remark, the aim of the above interpretation is to illustrate the general concept of a basin of attraction and should not provide an exact mathematical example. Actually, it is questionable if the resting state can be interpreted as a fixed point (with measure of zero) or should rather be modeled as an extended attractor 4 Furthermore, strictly speaking the discussed resting state is not stable under biological considerations, since cardiac muscle cells will initiate excitation waves by themselves if the external stimulation by the sinus rhythm is missing due to some reason. However, the state space sketched in Fig. 2.12 provides a basic picture, which will be used to illustrate the scientific objective, the main concepts, and the obtained results of each study of this thesis.

\section{Chaotic Attractor}

Similar to the fixed point, the chaotic attractor (also called strange attractor) is a special case of a general attractor, too. The dynamics of a trajectory inside the attractor is chaotic, which manifests in the sensitivity of initial conditions. This behavior can be quantified by the calculation of the leading Lyapunov exponents (section 2.2.4). A chaotic attractor is the topological object which is responsible for a permanent chaotic dynamics (in comparison to transient chaos, see below). It has a fractal structure, which can be estimated by e.g. the Kaplan-Yorke dimension (section 2.2.4). A specific trajectory comes arbitrarily close to each point of the attractor. As an example, a chaotic attractor can be found in the twodimensional Hénon map [33] in a specific parameter regime. The Hénon map is discussed extensively in the study of section 3.2 on page 76 .

\section{Repeller}

A repeller is similar to an attractor, whereas it has a repelling effect on neighboring trajectories. In some cases, a chaotic repeller can be responsible for chaotic transients, thus chaotic dynamics with a finite duration, followed by a non-chaotic behavior, governed by e.g. another attractor.

\section{Chaotic Saddle}

Chaotic saddles are invariant sets of the state space, which are besides chaotic repellers responsible for the occurrence of chaotic transients. They can be characterized by their influence on neighboring trajectories. In contrast to chaotic repellers which repel all close trajectories, chaotic saddles do have both, attracting and repelling directions. These directions are actually hypersurfaces in the state space and are denoted as the stable manifold and the unstable manifold. The stable manifold of a fixed point $\mathrm{x}^{*}$ is defined as all points $\mathrm{x}$ which will approach $\mathrm{x}^{*}$ as $t \rightarrow \infty$, whereas the unstable manifold is defined as the points $\mathrm{x}$ which will approach $\mathrm{x}^{*}$ as $t \rightarrow-\infty$. They are exemplary denoted in Fig. 2.13 in orange (stable manifold) and green (unstable manifold), respectively.

\footnotetext{
${ }^{4}$ The main question here, is how the state of the system should be parametrized by the model, since this parametrization defines the state space at the end.
} 


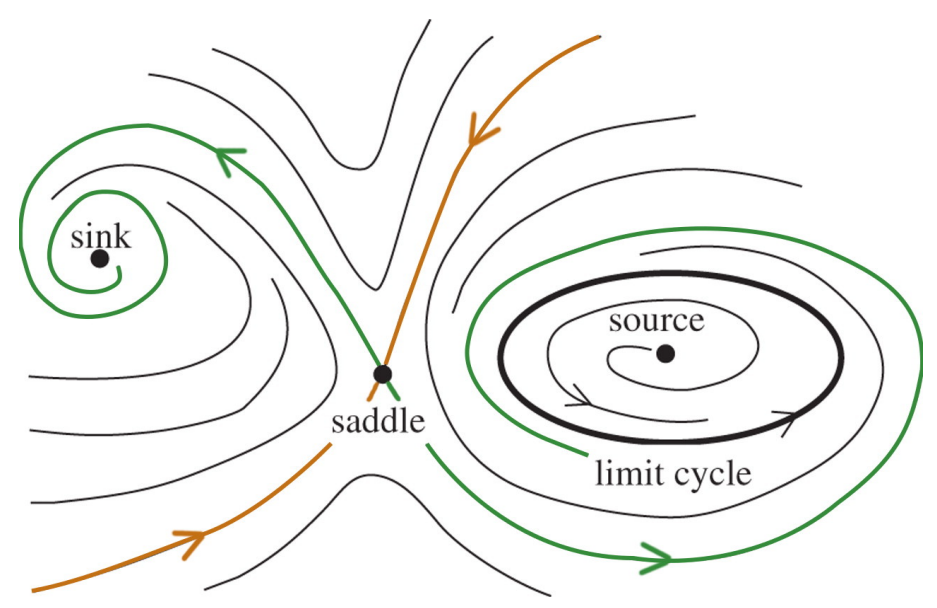

Figure 2.13: A saddle point in the state space. The saddle point is defined by the intersection of the stable manifold (orange) and the unstable manifold (green) of the fixed point. In this sketch, the saddle point coexists with a sink (stable fixed point) and a source (unstable fixed point), as well as a stable limit cycle. Republished with permission of Royal Society, from [34]; permission conveyed through Copyright Clearance Center, Inc.

A chaotic saddle is defined by the intersections of the stable and unstable manifold [35]. This behavior is depicted in Fig. 2.14. If the stable manifold and the unstable manifold intersect at one point, they must do so infinitely many times, since if one point is part of both manifolds, also the image and the preimage of the point have to be part of both manifolds. This results in a complex intertwined structure of both manifolds.

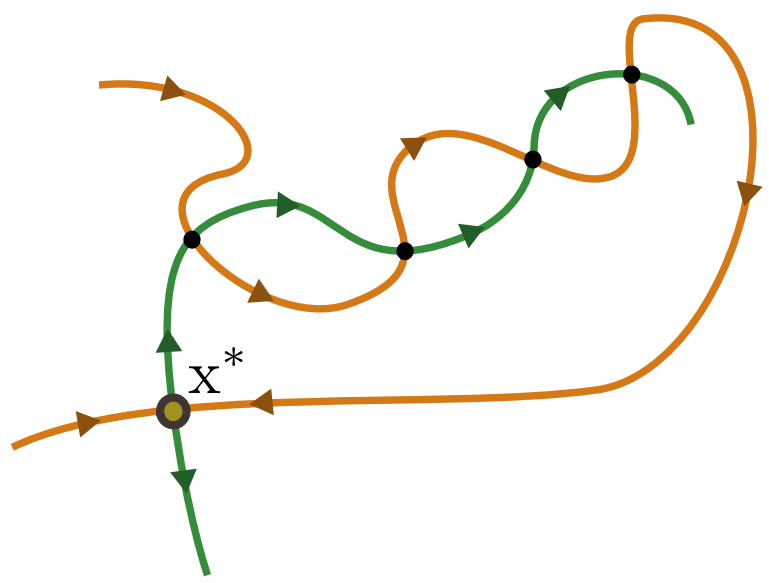

Figure 2.14: The intersection of the stable and the unstable manifold of a fixed point. The stable (orange) and unstable manifold (green) of a fixed point $\mathrm{x}^{*}$ are shown. The intersections of both manifolds (black circles) define the chaotic saddle.

In contrast to the transient chaotic dynamics governed by a repeller, long-living trajectories can in the case of a chaotic saddle initiate far away from the saddle. If they start in the proximity of the stable manifold, they are first attracted to the saddle. Since the repeller only has nonattracting directions, long-living episodes can only originate from the vicinity of the repeller. However, although a chaotic saddle has also attracting directions, the repeller 
and the chaotic saddle are both globally non-attracting sets, since all typical trajectories will depart after a finite transient time. Mathematically, the chaotic saddle itself is an invariant set with a corresponding infinite lifetime, but since its measure is zero, one will in practice, for example in numerical simulations, never reach such a trajectory with sustained chaos. Thus, although the chaotic saddle is the set which governs the dynamics, typical trajectories will in practice never reach the set itself, and the influence on the dynamics is mainly due to the attracting and non-attracting effect on neighboring trajectories.

Beside chaotic repellers, chaotic saddles are the sets in state space which are responsible for transient chaotic dynamics. They typically coexist with other topological objects in the state space, as for example an attractor or a limit cycle (as sketched in Fig. 2.13). Trajectories starting in the proximity of the saddle typically show chaotic dynamics for a transient time, until they reach these objects and as a consequence finish the chaotic dynamics. The length of the chaotic episode also depends sensitively on the initial condition. Chaotic transients

play a major role in studies of this thesis (e.g. section 3.1 on page 68 or section 3.2 on page 76. That is why the phenomenon of transient chaos will be discussed in more detail in the next section.

\subsubsection{Transient Chaos}

In the previous section, it was shown that repellers and chaotic saddles are the underlying structures which are responsible for the occurrence of transient chaos. With this observation, one can define transient chaos in particular in contrast to sustained chaotic dynamics as the form of chaos which is governed by nonattracting chaotic sets.

Transient chaotic dynamics can appear in many different systems, like turbulence [36], ecology [37, or neural networks [38, 39, 40]. The end of a chaotic episode is from here on denoted as the collapse or self-termination of the dynamics. A first distinction between permanent and transient chaos can be made referring to the lifetime $T$ of the dynamics, which is infinite for sustained chaos and has a finite duration in the latter case. In practice, this differentiation is not trivial, since the observation time of the dynamics is always finite. Thus, when observing a chaotic time series extracted from experimental data, one can technically not exclude that chaos is transient here, although during the observation time the dynamics is permanently chaotic [41].

\section{Properties of Chaotic Transients}

Ying-Cheng Lai and Tamás Tél characterize transient chaos by the following properties [41]:

1. The dynamics of an exemplary initial condition shows chaotic behavior, until a certain point in time where the dynamics switches to another quantitative behavior in many cases non-chaotic. The lifetime of a specific trajectory depends due to the chaotic properties sensitively on the initial condition.

2. There exists a smooth probability distribution to find an initial condition corresponding to a lifetime larger than $T, P(T)$. This distribution converges to 0 , for $T \rightarrow \infty$. 
(a)

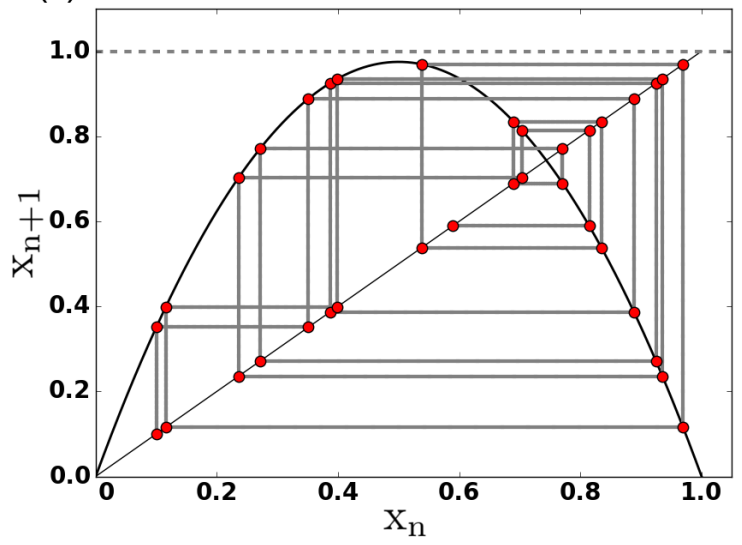

(b)

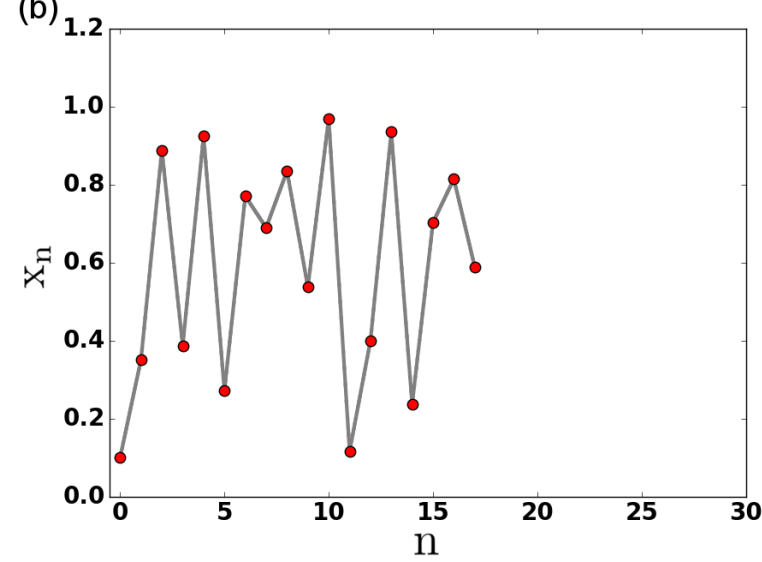

Figure 2.15: Sustained chaotic dynamics of the logistic map. The trajectory of an exemplary trajectory $\left(x_{0}=0.1\right)$ is shown in a Coweb Plot (a) for $r=3.9$. The corresponding time series is shown in (b). With $r_{\mathrm{c}} \leq 4$ the chaotic dynamics is persistent.

3. Theoretically, there exist trajectories with an infinite lifetime (associated with the saddle itself). Since the Lebesgue measure of the set of these initial conditions is zero, in practice the transient lifetime of a randomly chosen initial condition is always finite.

4. In comparison to the dynamics governed by a chaotic attractor (where an arbitrary small perturbation of the parameters can lead the system into a periodic attractor), transient chaos is in this sense robust against small parameter perturbations.

Although chaotic transients in spatially extended high-dimensional systems play the major role in the studies of this thesis, possible underlying mechanisms can be studied more easily in simple systems like low-dimensional maps. As an example, the transition from persistent chaotic dynamics to transient chaos is presented in the following for the logistic map.

\section{From Sustained to Transient Chaos: The Logistic Map}

The logistic map is a simple one-dimensional map, where basic principles can be studied. It is defined by:

$$
x_{\mathrm{n}+1}=r x_{\mathrm{n}}\left(1-x_{\mathrm{n}}\right) \text {, }
$$

where $x_{\mathrm{n}}$ is a number between 0 and 1 , and $r$ is the only parameter of the map.

With $r=3.9$, the system exhibits persistent chaotic dynamics as shown for an exemplary initial condition $x_{0}=0.1$ in a Coweb plot, together with the corresponding time series (Fig. 2.15(a) and (b), respectively). If $r$ exceeds $r_{\mathrm{c}}=4$, an "exit" of the chaotic regime is created, and thus the chaotic dynamics becomes transient. In Fig. 2.16 this behavior is depicted for the same initial condition as in Fig. 2.15. When the trajectory "hits" the exit window (around $x_{\mathrm{n}}=0.5$ ) it leaves the chaotic regime and diverges to $-\infty$ (highlighted in red). 
(a)

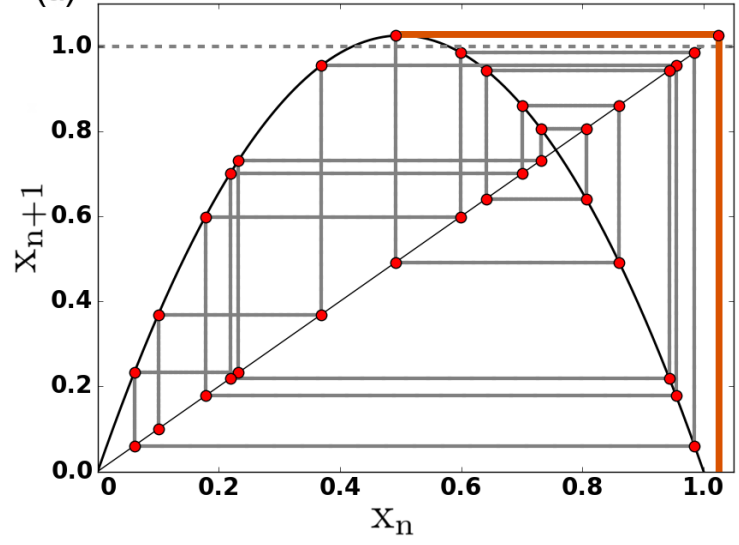

(b)

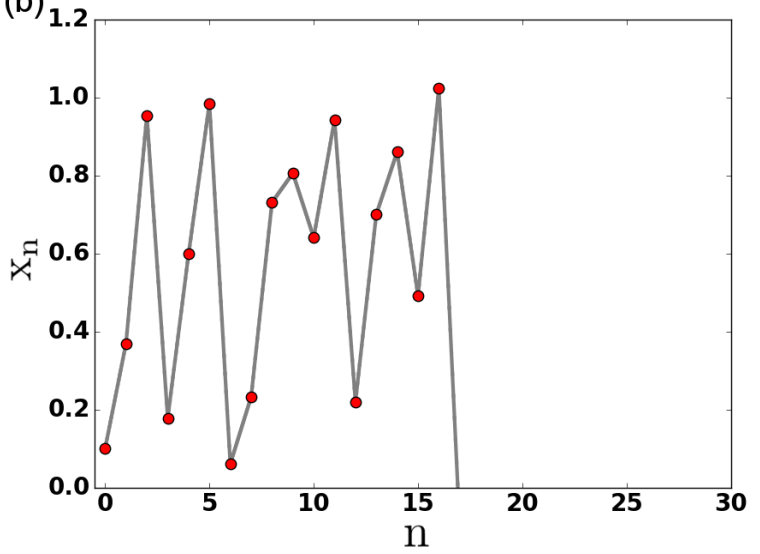

Figure 2.16: Transient chaotic dynamics of the logistic map. The trajectory of an exemplary trajectory $\left(x_{0}=0.1\right)$ is shown in a Coweb Plot (a) for $r=4.1$. The corresponding time series is shown in (b). With $r_{\mathrm{c}}>4$ the chaotic dynamics is transient, since points which map into a window around $x_{\mathrm{n}}=0.5$ diverge. The final part of the trajectory where it passes this window is depicted in orange in (a).

Although the specific mechanisms which govern the end of chaotic episodes in low-dimensional systems like the logistic map can not be generalized to high-dimensional systems, investigating these simple maps allows the study of basic effects which may also play a role in the complex systems.

More generally apart from the identification of individual mechanisms which lead to the collapse of the dynamics, chaotic transients can, in a first step, be described and characterized by other, more descriptive properties.

\section{Characterizing Chaotic Transients}

In high-dimensional systems, the investigation of a single trajectory provides usually only limited insight into the dynamics of the underlying objects (non-attracting sets). For a global qualitative description, a larger number of initial conditions is more appropriate in order to achieve a dense sampling of the relevant regions in the state space. Hence, from a large statistics averaged quantities can be derived, which characterize the dynamics.

A useful quantity is the escape rate $\kappa[42$, the averaged rate at which trajectories leave the region of the state space which governs the chaotic dynamics. Assuming a certain number of initial conditions $N_{\mathrm{Ch}}\left(t_{0}\right)$ in the state space which exhibit chaotic dynamics. As time goes on, the number of these trajectories $N_{\mathrm{Ch}}(t)$ which are still chaotic at a point in time $t$ (thus the number of trajectories which have not yet collapsed) decreases. In general, for large $t$ an exponential decay can be observed:

$$
N_{\mathrm{Ch}}(t) \sim \exp (-\kappa t) .
$$

In practice, the created initial conditions are not necessarily uncorrelated. These correlations 
of initial conditions can possibly cause a spoiling of the exponential shape of Eq. (2.4) for small $t$. For a reasonable estimate of $\kappa$, a transient evolution time should therefore be discarded. In chaotic systems, the inverse of the biggest Lyapunov exponent (introduced in the next section) can be used to estimate a lower limit for this transient time, since the biggest Lyapunov exponent determines how fast two trajectories depart from each other.

This consideration is also relevant for the determination of the average lifetime $\langle T\rangle$ of the transient chaotic dynamics. Instead of calculating $\langle T\rangle$ from averaging over the single lifetimes of the initial conditions, the average lifetime is approximately proportional to the inverse of the escape rate

$$
\langle T\rangle \approx \frac{1}{\kappa}
$$

The average lifetime of a chaotic system can be highly relevant for practical implications of the specific system (for example in the study of section 3.1 on page 68 ).

Finally, it is noteworthy that in high-dimensional systems it is very difficult or often practically impossible to determine whether the dynamics is governed by only one saddle and a corresponding escape rate, or by plenty of non-attracting sets. In the case of, for example, two coexistent saddles the global escape rate is composed by two escape rates, which are associated to the two saddles.

\section{Supertransients}

Supertransients appear in spatially-extended systems and can be characterized by a rapid growth of the average lifetime $\langle T\rangle$ with the system size. Two subgroups can be distinguished with the following properties [41, 43]:

- Type-I: Characterized by a continuous decrease of the number of "defects" The dynamics is denoted nonstationary for this reason, since the state of the dynamics can be read off the number of these "defects". The scaling of the average lifetime dependent on the system size $L$ is determined by

$$
\langle T\rangle \sim L^{\beta},
$$

where $\beta$ is a positive parameter.

- Type-II: In systems of this group, the number of "defects" fluctuates during the chaotic episode. The collapse of the dynamics is therefore spontaneous and the dynamics is called quasi-stationary. In contrast to the power law scaling of type-I supertransients, the average lifetime increases exponentially with the system size here:

\footnotetext{
${ }^{5}$ The exact meaning of a "defect" needs to be specified by the corresponding dynamics of the system. In the case of cardiac dynamics, the number of spiral waves in two-dimensional systems could for example be considered as "defects".
} 


$$
\langle T\rangle \sim \exp \left(a L^{\gamma}\right)
$$

with $\gamma$ a positive exponent and $a$ a system specific parameter.

In two studies of this thesis we identify type-II supertransients, investigate in detail the underlying mechanisms (section 3.1 on page 68 , and extend the general understanding of the final phase before the collapse of the dynamics in these systems (section 3.2 on page 76.)

\subsubsection{Analyzing Chaotic Dynamics}

A characteristic feature of chaotic systems is the exponentially fast separation of (initially close) trajectories. The theory of Lyapunov exponents and their corresponding Lyapunov vectors quantifies this behavior and reveals details about the topological structure of the state space. Broadly speaking, Lyapunov exponents describe the growth rates of (initially small) perturbations applied to a generic trajectory of the dynamical system. Calculating the Lyapunov exponents of a dynamical system is a powerful and one of the most common tools in nonlinear dynamics which can be applied to a broad class of systems. In this section the mathematical foundation of Lyapunov exponents is presented, whereas details about their numerical computation is discussed in section 2.4 .5 on page 60 .

For the following considerations, a dynamical system described by an ordinary differential equation

$$
\frac{\partial \mathbf{x}(t)}{\partial t}=\mathbf{f}(\mathbf{x}(t), t)
$$

is assumed, where $\mathbf{x} \in \mathbb{R}^{m}$ denotes an m-dimensional state vector which characterizes the state of the system, and $\mathbf{f}(\mathbf{x}(t), t)$ describes the dynamical evolution, which can depend also explicitly on time, in addition to the time dependent state vector.

\section{The largest Lyapunov Exponent}

The largest Lyapunov exponent can intuitively be understood by applying a small perturbation $\delta \mathbf{x}_{0}$ to a generic initial condition $\mathbf{x}\left(t_{0}\right)$ (Eq. (2.9)):

$$
\begin{aligned}
\mathbf{x}\left(t_{0}\right) \rightarrow \mathbf{x}^{*}\left(t_{0}\right) & =\mathbf{x}\left(t_{0}\right)+\delta \mathbf{x}\left(t_{0}\right) \\
\delta \mathbf{x}(t) & =\mathbf{x}^{*}(t)-\mathbf{x}(t)
\end{aligned}
$$

The temporal evolution of the perturbation $\delta \mathbf{x}(t)$ (Eq. 2.10) can then provide indications, whether the dynamics is chaotic or not 6

\footnotetext{
${ }^{6}$ Actually, a positive Lyapunov exponent alone is in general not sufficient for the identification of chaos.
} 


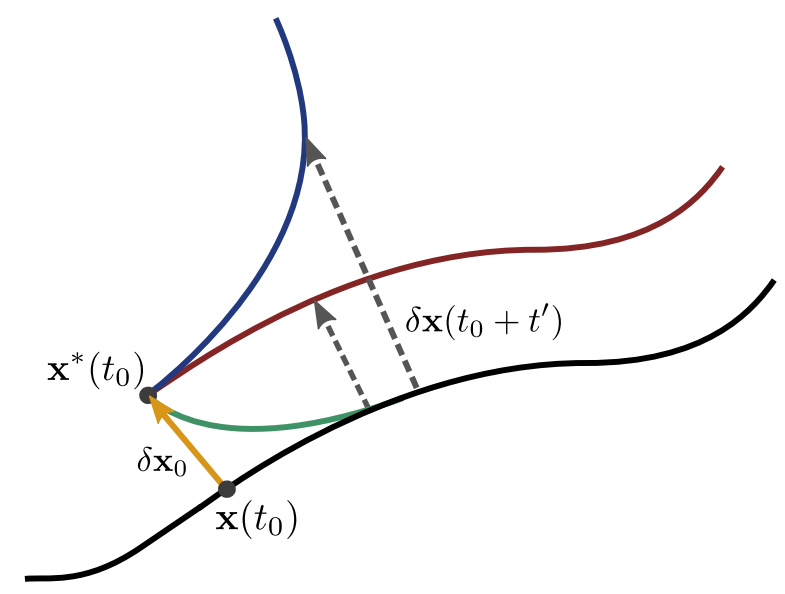

Figure 2.17: Perturbation of a trajectory in state space. A perturbation $\delta \mathbf{x}_{0}$ is applied to a trajectory at a specific point in time $\mathbf{x}\left(t_{0}\right)$. The length of the perturbation $\mathbf{x}(t)$ (the difference between the unperturbed and the perturbed trajectory) can grow (blue), shrink (green), or stay constant in time (red).

$$
\frac{|\delta \mathbf{x}(t)|}{\left|\delta \mathbf{x}_{0}\right|} \approx e^{\lambda t}
$$

In Eq. 2.11 $\lambda$ is called the (largest) Lyapunov exponent, which is used to quantify the growth rate of the perturbation. In a first analysis, one can roughly distinguish between a positive, a negative, and an exponent equal to zero. In Fig. 2.17, these three cases are schematically shown: Decreasing perturbations (green trajectory) yield a negative Lyapunov exponent, whereas a positive exponent describes trajectories which separate from each other (blue trajectory). An exponent equal to zero describes a trajectory which has a constant distance to the unperturbed one.7

The expression from Eq. 2.11) can be generalized to the definition of the largest Lyapunov exponent $\lambda$, using an infinitely small perturbation and an infinite evolution time:

$$
\lambda=\lim _{t \rightarrow \infty} \lim _{\left|\delta \mathbf{x}\left(t_{0}\right)\right| \rightarrow 0} \frac{1}{t} \ln \frac{|\delta \mathbf{x}(t)|}{\left|\delta \mathbf{x}\left(t_{0}\right)\right|} .
$$

Oseledets theorem [44] justifies that the exponent in Eq. (2.12) is not time dependent, and thus it is a quantity describing the whole system and not just a specific trajectory. Furthermore, it guarantees that Eq. (2.12) holds for almost all initial conditions $\mathbf{x}\left(t_{0}\right)$.

\section{Lyapunov Vectors}

Later in this section we will see, that not only one Lyapunov exponent $\lambda$ can be determined,

\footnotetext{
${ }^{7}$ Note that one of the Lyapunov exponents (not necessarily the largest one) is always zero, since it belongs to a perturbation heading into the direction of the evolution of the unperturbed trajectory. In this sense, the perturbation shifts the original trajectory only in time. The perturbed trajectory is in the following evolution a finite amount of time (and thus space) ahead of the original one, but never separates from it.
} 
but there exists a whole spectrum of exponents $\lambda_{i}$. Furthermore, Lyapunov vectors can be associated with each Lyapunov exponent. These vectors are time dependent, and can be understood as specific perturbations of the trajectory, which will grow or shrink with a corresponding Lyapunov exponent (see Eq. (2.11)). They provide an orthonormal basis in the tangent space, the space of infinitesimal perturbations. The dimension of the tangent space and thus the number of Lyapunov exponents and Lyapunov vectors is equal to the dimension of the system itself. One can show, that with the help of the Lyapunov vectors one can define specific subspaces of the tangent space. Perturbations which belong to these subspaces will grow/shrink with the corresponding Lyapunov exponent. We will see later in this section, that almost every perturbation will grow/shrink with the largest Lyapunov exponent, that means the subspace corresponding to the largest Lyapunov exponent has a measure of $\mathbb{R}^{m}$ [45]. This is the reason why in practice, Eq. 2.12) can be used for the calculation of the largest Lyapunov exponent using basically any kind of perturbation.

\section{The Lyapunov Spectrum}

As discussed in the previous section, the Lyapunov exponents of a system $\lambda_{i}$ correspond to Lyapunov vectors, which are located in the tangent space, describing the perturbations which then grow with the corresponding exponent. In order to specify the Lyapunov vectors and the related subspaces of the tangent space, the temporal evolution of perturbation vectors needs to be derived. Equation 2.13 presents a Taylor expansion of Eq. 2.8, where a differential equation for small perturbations $\delta \mathbf{x}(t)$ can be approximated (Eq. (2.14)) using the Jacobi matrix $\mathbf{J}_{\mathbf{f}}$ of $\mathbf{f}$ :

$$
\begin{aligned}
\frac{\partial(\mathbf{x}(t)+\delta \mathbf{x}(t))}{\partial t} & =\mathbf{f}(\mathbf{x}(t)+\delta \mathbf{x}(t))=\mathbf{f}(\mathbf{x}(t))+\mathbf{J}_{\mathbf{f}} \delta \mathbf{x}(t)+\mathcal{O}\left(\delta \mathbf{x}^{2}\right) \\
\Rightarrow \frac{\partial \delta \mathbf{x}(t)}{\partial t} & \approx \mathbf{J}_{\mathbf{f}} \delta \mathbf{x}(t)
\end{aligned}
$$

The fundamental matrix $\mathbf{M}$ is then defined by the equation

$$
\frac{\partial \mathbf{M}}{\partial t}=\mathbf{J}_{\mathbf{f}} \mathbf{M}
$$

where the initial condition $\mathbf{M}(0)=\mathbb{1}$ can be used. With the help of $\mathbf{M}$ the linear propagator $\mathcal{F}\left(t_{1}, t_{2}\right)$ can be defined by

$$
\mathcal{F}\left(t_{1}, t_{2}\right)=\mathbf{M}\left(t_{2}\right) \mathbf{M}\left(t_{1}\right)^{-1}
$$

which evolves perturbations from time $t_{1}$ to $t_{2}$ in tangent space:

$$
\delta \mathbf{x}\left(t_{2}\right)=\mathcal{F}\left(t_{1}, t_{2}\right) \delta \mathbf{x}\left(t_{1}\right)
$$


Using Eq. 2.17), the growth of the Euclidean norm of perturbation vectors $\delta \mathbf{x}\left(t_{1}\right)$ is given by $\mathcal{F}\left(t_{1}, t_{2}\right)^{T} \mathcal{F}\left(t_{1}, t_{2}\right)$, where $\mathcal{F}\left(t_{1}, t_{2}\right)^{T}$ is the transposed of $\mathcal{F}\left(t_{1}, t_{2}\right)$. The Lyapunov exponents are only defined in the large time limit (see Eq. (2.12). Therefore it is reasonable to define the far-future operator [45]:

$$
\mathbf{W}(t)=\lim _{t_{2} \rightarrow \infty}\left(\mathcal{F}\left(t, t_{2}\right)^{T} \mathcal{F}\left(t, t_{2}\right)\right)^{1 /\left(2\left(t_{2}-t\right)\right)}
$$

whose eigenvectors $\varphi_{i}(t)$ are denoted as Lyapunov vectors 8 The Lyapunov vectors form an orthonormal basis of the tangent space. Furthermore, the Lyapunov exponents $\lambda_{i}$ are obtained by taking the logarithm of the eigenvalues of $\mathbf{W}(t)$. Note, that the Lyapunov vectors depend like $\mathbf{W}(t)$ on time, whereas the Lyapunov exponents do not [44, which means that they are invariant. A possible degeneracy of the Lyapunov exponents is neglected here, since we focus on the general understanding. For more details see [45].

With the definition of the Lyapunov vectors, we can derive an expression for the Lyapunov exponents which is essential for the numerical computation in the end. For this purpose, subspaces $S_{j}(t)$ of the tangent space can be defined using the span of the (orthonormal) Lyapunov vectors by the following properties:

$$
\begin{aligned}
S_{j}(t) & =\operatorname{span}\left\{\varphi_{\lambda_{i}} \mid i=j, j+1, \ldots, m\right\}, \\
S_{s+1}(t) & =\emptyset, \\
S_{s}(t) & \subset S_{s-1}(t) \subset \ldots \subset S_{1}(t)=\mathbb{R}^{m},
\end{aligned}
$$

where the subspace $S_{j}(t)$ is defined by the span of all but the first $j-1$ Lyapunov vectors. By specifying the subspace for possible perturbations $\delta \mathbf{x}(t)$, one can then formulate an expression for the Lyapunov exponents using the linear propagator:

$$
\lambda_{i}=\lim _{t_{2} \rightarrow \infty} \frac{1}{t_{2}-t_{1}} \ln \left\|\mathcal{F}\left(t_{1}, t_{2}\right) \delta \mathbf{x}\left(t_{1}\right)\right\| \quad \delta \mathbf{x}\left(t_{1}\right) \in S_{j}\left(t_{1}\right) \backslash S_{j+1}\left(t_{1}\right)
$$

The construction of the proper subspace $S_{j}\left(t_{1}\right) \backslash S_{j+1}\left(t_{1}\right)$ for the determination of a certain Lyapunov exponent $\lambda_{i}$ is quite intuitive. This decomposing of the perturbation space into proper subspaces is called Oseledec splitting [44, 46]. As an example, let us assume the calculation of a specific Lyapunov exponent $\lambda_{k}$ with $\lambda_{1}>\lambda_{2}>\cdots>\lambda_{k}>\cdots>\lambda_{m} 9$. First of all, $S_{k}(t)$ excludes all perturbation vectors which have components belonging to larger Lyapunov exponents which would then grow corresponding to the larger Lyapunov exponents. In Fig. 2.18 $S_{k}$ is sketched as the hyperplane in the tangent space, spanned by the Lyapunov vectors $\varphi_{l}$ with $l>k-1$ (in the sketch, only $\varphi_{k}$ and $\varphi_{k+1}$ are shown). Then, for the determination of the proper subspace $S_{k} \backslash S_{k+1}\left(t_{1}\right)$ corresponding to $\lambda_{k}$,

\footnotetext{
${ }^{8}$ In particular, they are denoted as forward Lyapunov vectors, since the far-future operator considers the evolution into the future (forward direction). For more details, see [45].

${ }^{9}$ Also here, for the sake of clarity we neglect degeneracy.
} 


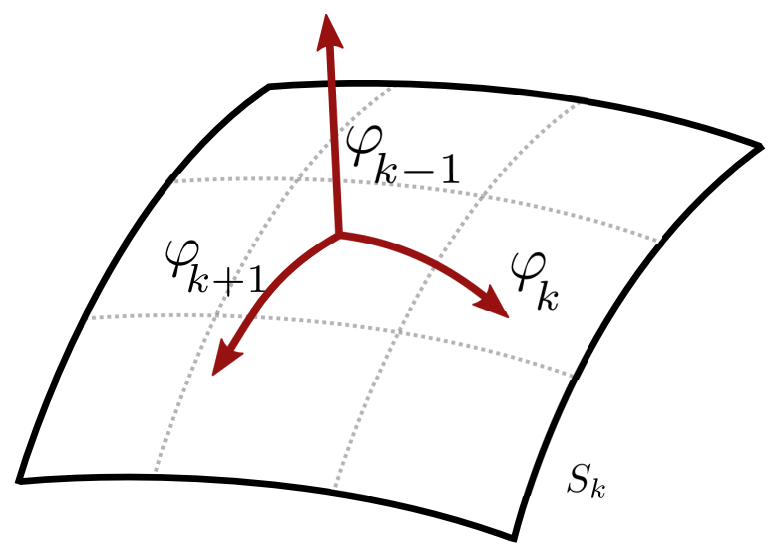

Figure 2.18: Lyapunov Vectors in tangent space. Three Lyapunov vectors are sketched here $\varphi_{k-1}, \varphi_{k}$ and $\varphi_{k+1}$, where the latter ones form the subspace $S_{k}$, sketched as a hypersurface here. Note, that naturally only three dimensions are shown in the sketch, whereas in most cases the perturbation space can be very high-dimensional.

directions which are orthogonal to $\varphi_{k}$, and thus have no component into the direction of $\varphi_{k}$, need to be extracted. In the sketch, the subspace into the direction of $\varphi_{k+1}$ needs to be subtracted, for example and also all the directions corresponding to $\varphi_{l}$ with $l>k+1$, not plotted here. Practically that means, almost every perturbation vector in the subspace $S_{k}$ (sketched in Fig. 2.18 as the hypersurface) has a component of $\varphi_{k}$ and will therefore grow/shrink with $\lambda_{k}$.

In the specific case of $\lambda_{1}$, the subspace $S_{1}\left(t_{1}\right) \backslash S_{2}\left(t_{1}\right)$ consists of $\mathbb{R}^{m}$, but excluding the subspace which is orthogonal to $\varphi_{1} \sqrt{10}$ Actually, this excluded subspace has a measure of zero, which means that in practice, almost every perturbation vector will grow with the largest Lyapunov exponent.

The fact that the perturbation vectors, which grow with a Lyapunov exponent which is not equal to the largest one, have to be orthogonal to all Lyapunov vectors corresponding to larger exponents is crucial for the numerical implementation.

\section{Lyapunov Exponents in Systems with Discrete Time}

Equation 2.22 provides an expression for the calculation of the Lyapunov spectrum. In the case of systems with a discrete time with the time step $d t^{11}$ the linear propagator can be rewritten by the Jacobi matrix $\mathbf{J}_{f}$, as proposed by Benettin et al. [46]:

$$
\mathcal{F}\left(t_{1}, t_{2}\right)=\prod_{i=1}^{k} \mathbf{J}_{f}
$$

where $k$ is determined by the number of discrete time steps:

\footnotetext{
${ }^{10}$ Remember that the Lyapunov vectors $\varphi_{i}$ are orthonormal.

${ }^{11}$ In practice, this includes also continuous systems, described by differential equations which are solved using a numerical scheme. Thus they are quasi discrete.
} 


$$
k=\frac{t_{2}-t_{1}}{d t}
$$

Since all studies of this thesis present the results of numerical simulations using a discrete time stepping scheme, Eq. 2.22 can be used in combination with Eq. 2.23) for the calculation of Lyapunov exponents, only by the knowledge of the Jacobi matrix. The numerical implementation of the algorithm for computing the Lyapunov exponents in numerical simulations is discussed in section 2.4 .5 on page 60

Although Lyapunov exponents are defined in the infinite time limit, they can in most cases also be used to characterize the chaotic properties of chaotic transients. The main criterion for the proper application is, that the chaotic episodes last long enough to allow the Lyapunov exponents to converge. This rather technical issue is discussed in section $2.4 .5 \mathrm{on}$ page 60 in detail.

\section{The Kaplan-Yorke Dimension}

The geometrical structure of chaotic attractors can be very complex (e.g. fractal) and hard to describe. However, measures are needed, in order to compare geometrical quantities of different attractors, like for example their dimension. In the following, the Kaplan-Yorke dimension is presented, which can be calculated based on the Lyapunov spectrum. It was introduced by James L. Kaplan and James A. Yorke [47], who claimed that it is equal to the information dimension ${ }^{12}$ for typical systems (this is shown for two-dimensional maps in [49] and [50]).

The Kaplan-Yorke dimension is defined as

$$
D_{\mathrm{KY}}=k+\sum_{i=1}^{k} \frac{\lambda_{i}}{\left|\lambda_{k+1}\right|},
$$

with $k$ chosen such that

$$
\sum_{i=1}^{k} \lambda_{i} \geq 0 \quad \text { and } \quad \sum_{i=1}^{k+1} \lambda_{i}<0 .
$$

\footnotetext{
${ }^{12}$ The information dimension is another approach to estimate the dimension of an attractor [48. The state space is separated into small boxes with the size $\epsilon$. Then, the information dimension can be thought of the number of boxes which are needed to cover the attractor (in addition, each box is weighted by the frequency that the points in the box are visited), in the limit of $\epsilon \rightarrow 0$.
} 
Although counter examples have been found [51, the Kaplan-Yorke dimension is a reasonable approximation for the information dimension in most cases. One remarkable feature of the Kaplan-Yorke dimension is that it relates the Lyapunov spectrum of a system, which is a property derived from the dynamics, to geometrical characteristics. Furthermore, in non-attracting chaotic sets, the Kapan-Yorke dimension is related to the average transient lifetime of the system [52, 53]. This feature will also be shown in the study of section 3.1 on page 68 .

\subsection{The Role of Numerical Simulations}

With four parameters I can fit an elephant, and with five I can make him wiggle his trunk. John von Neumann

Utilizing numerical algorithms in the field of nonlinear dynamics is in most cases inevitable, since the underlying equations which describe the dynamics of the system can usually not be solved analytically. For example, simple generic systems (e.g. low-dimensional maps) are often used, to study basic dynamics and effects. Here, the equations or maps themselves are the objects of interest. In other cases, differential equations are derived on the basis of experimental observations, in order to model the real system (e.g. the Lorenz system [54] describing atmospheric convection).

\subsubsection{Interplay of Experiments and Numerical Simulations}

In the field of cardiac dynamics numerical simulations play a significant role concerning the understanding of basic mechanisms and features of the dynamics of the cardiac muscle. The strong demand for simulation studies has different reasons: Current methods and techniques used in ex-vivo animal heart experiments are able to highlight the electrical wave dynamics on the surface of the heart. The optical mapping technique uses for this purpose fluorescent dyes which are sensitive to voltage or the local calcium concentration [55]. However, the electrical activity in the bulk of the cardiac muscle remains mostly unknown. Also, in the experiment different hearts can exhibit distinct varieties e.g in terms of anatomical sizes or the ability to induce ventricular fibrillation. For ethical reasons the number of experiments involving the sacrifice of an animal should be as low as possible. Thus, experiments using the heart of an animal can also be considered as probing or sampling a very high-dimensional (parameter) space with very limited data points. Still, for the validation of a scientific hypothesis, an adequate number of measurements is needed. Hence, numerical simulations are the ideal environment to test first ideas and hypothesis, which can later be validated in experiments.

Compared with the experimental limitations, in numerical simulations the quantity of results is mainly limited by the computational power. Here, the full information about the state of the system is available at any time, and the exact same simulation can be reproduced if this is desired. In addition, the researcher can interact with the system in a way which is perhaps not (yet) possible in the real world, thus new methods can be tested here. 
The overwhelming 13 complexity of a real heart can be broken down to the main governing processes. The governing features, mechanisms and their interaction can be studied separately (e.g. geometrical effects, the influence of heterogeneities or the role of diseases), and specific regions in the parameter space can be (quasi continuously) analyzed.

However, in order to ensure that the performed simulations reproduce to some extent the dynamics of a real heart, two key features are essential:

- The interaction with the experiments (and with the experimentalists) is crucial: In most cases the motivation for a simulation study originates from experimental results. In addition, each simulation should provide quantities which can be linked (or gauged) to corresponding observables from the experiment (e.g. length/time scales of action potential durations or equivalent numbers of spiral waves in the simulation domain and inside the heart). Only this connection justifies a possible validity of the numerical results.

- As already stated before, different mechanisms can be investigated separately in simulations. The process which simplifies the original complexity to its key features which should be investigated is called modeling. This process is essential for the significance of a simulation study, and will be discussed explicitly in the next section.

\subsubsection{The Process of Modeling}

Creating a model means, to simplify the full complexity and diversity of functionalities of the real (biological) system, while reproducing essential features and dynamics. That means, the model can describe the real system at different levels of details, as illustrated by the lithographs Der Stier by Pablo Picasso (Fig. 2.19) 14

How the dynamics of the heart can be modeled will be discussed in the next sections, including for example the underlying differential equations describing the excitation wave propagation, but also the choice of the simulation domain (two-dimensional, three-dimensional, rectangular, realistic geometry, etc.). Today, there exist a variety of models of different levels of complexity, which aim at modeling the diverse mechanisms and dynamics of the heart. In theory, a numerical study could therefore take all the most sophisticated models present into account in order to create a simulation as realistic as possible. The upper limit for the complexity of the simulation is the computer power which is available.

However, we know from experiments that various hearts can be very different in their anatomical size and their dynamic behavior. Hence, modeling a specific real heart in every detail in simulations does not ensure that the obtained results are generally applicable (thus are valid for every heart). Rather, the scientific objective is often to find and investigate robust phenomena, which play a relevant role in most clinical cases. For this reason, simulations need to be performed, which cover a broad range of parameters. Concerning the limitation due to the computational cost it is therefore (in most cases) reasonable to perform

\footnotetext{
${ }^{13}$ At least for a physicist.

${ }^{14}$ Inspired by a talk of Peter Kohl at the 26th of January 2016, in the colloquium of the SFB 937 with the title Systems Biology of the Heart: Model or Muddle?.
} 

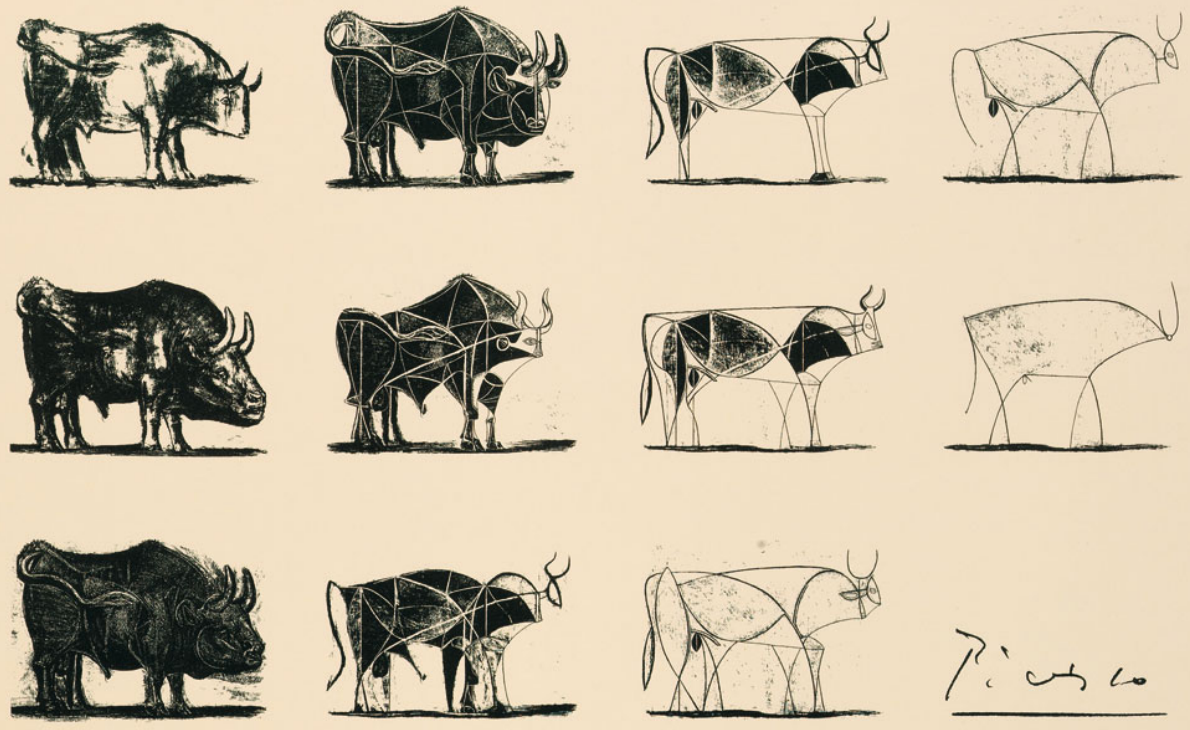

Figure 2.19: Pablo Picasso: Der Stier, Zustand I-XI, Lithographien, 1945-46. (C) Succession Picasso/VG Bild-Kunst, Bonn 2018, [56]. The differences between each drawing can be viewed as the process of modeling various levels of details of a complex object.

simulations including models of moderate levels of complexity but with varying parameters instead of very few realistic simulations 15

The governing question is, which dynamical models and features the simulation should comprise. The answer to this question is essential for the general field of numerical simulations of complex (biological) systems:

- The ingredients (the choice of the main features and models) of a numerical simulation should always be determined by the scientific objective of the study.

The above statement involves, that a numerical study with the purpose of investigating a certain scientific hypothesis should comprise primarily those dynamics and features (in particular the corresponding models) which are presumably relevant for the investigation of the objective.

\subsubsection{Designing a Simulation}

The conclusion from the previous section, that a numerical study should comprise those features which are relevant for the respective scientific objective, leads to the issue how to actually evaluate the relevance of a specific feature (e.g. whether to use a simple cell model, or a more sophisticated ionic model).

\footnotetext{
${ }^{15}$ In case of, for example, patient specific studies, this is of course not the case.
} 
The range of possible "ingredients" for a numerical simulation is broad. Potential features may, for example, be:

\section{The Simulation Domain}

Two-dimensional or three-dimensional domains. Realistic geometry or "generic" rectangular/cubic domain.

\section{The Cell Dynamics Model}

Simple (low-dimensional) models, or complex ionic cell models with various specific ion channel dynamics included.

\section{The Substrate's Heterogeneity and Anisotropy}

Homogeneous or heterogeneous tissue (e.g. inclusion of heterogeneities like Purkinje fibers). Isotropic or anisotropic properties. Spatial dependent parameters (like conductivity or refractory behavior).

\section{The Spatial Resolution of the Intracellular and Extracellular Domain}

Bidomain or monodomain description of the substrate.

\section{The Electro-Mechanical Coupling}

Possible decoupling of the mechanical and electrical dynamics.

The framework of a concrete simulation can be composed of the relevant ingredients of the above list. For example, when studying general features of spiral-spiral interaction, a simple cell model as the Fenton-Karma model (section 2.4.2 on page 48) can be used. Details about special ion channel dynamics could be negligible in this case.

However, the choice of the right ingredients to address a scientific objective is also always based on experimental experience. Motivated by the above considerations, a short justification for the used models and features is given for each study of this thesis, respectively (given in the introduction of each study).

\subsection{Numerical Simulations}

How the evolution of a complex biological system like the heart can be described mathematically was introduced in section 2.2 on page 25 using the theory of dynamical systems. Also, the significant role of the process of modeling was elucidated (section 2.3 on page 40 ). Now in this section, the concrete differential equations of the underlying models used in this thesis are derived. Furthermore, the numerical methods used to solve the equations and the numerical implementation of all other features of the simulations performed (including tools used for analyzing the numerical data) are shown.

The central element of all studies of this thesis is the simulation of the electrical wave propagation in cardiac tissue as an excitable medium. As discussed in section 2.1 on page 11 . 
this electrical signal triggers subsequently the mechanical contraction of the tissue, thus, by controlling the electrical domain also the mechanical behavior is determined. However, the direction of this causality is not purely unidirectional: stretch-activated ion channels (also called mechano-sensitive channels) can trigger an electrical action potential, induced by mechanical stretch [6]. Nevertheless, in this thesis this effect is neglected and we concentrate also for reasons of a reduced complexity on the electrical dynamics of the system. However, the actual role of stretch-activated channels during cardiac arrhythmias remains unclear [57.

As a remark, the major part of the studies of this thesis present the simulation results of excitable media. In the study of section 3.2 on page 76 , also other models are investigated in order to apply the findings on a more general class of systems. However, since these models are only relevant for this specific study, they are introduced and described there.

\subsubsection{From Bidomain to Monodomain Model}

As a generalization of the cable equation [58, the bidomain model is a widely used model for simulations of the action potential propagation in cardiac tissue. As its name already suggests, the model describes two domains, the extracellular domain, and the intracellular domain of the tissue, separated by the cell membrane (section 2.1 on page 11). In particular, the underlying equations determine the evolution of the intra- and extracellular potentials, $V_{\mathrm{i}}$ and $V_{\mathrm{e}}$, respectively. For the sake of completeness, we consider here the case of cardiac tissue (denoted from now on by $\mathbb{T}$ ) surrounded by another medium (e.g. a surrounding tissue or bath), denoted from now on with $\mathbb{M}$. Hence, the variable $V_{0}$ describes the electrical potential in the surrounding medium. We first derive the general bidomain equations including boundary conditions here, simplify the equations to the monodomain approach and discuss under which conditions this reduction is valid.

\section{The Bidomain Model}

Inside the tissue, $V_{\mathrm{i}}$ and $V_{\mathrm{e}}$ represent the electrical potential at each point of the domain ${ }^{16}$ The electrical field $\mathbf{E}_{\mathrm{i} / \mathrm{e}}$ in each domain is then given by

$$
\begin{aligned}
& \mathbf{E}_{\mathrm{i}}=-\vec{\nabla} V_{\mathrm{i}}, \\
& \mathbf{E}_{\mathrm{e}}=-\vec{\nabla} V_{\mathrm{e}} .
\end{aligned}
$$

By introducing the conductivity tensor $\boldsymbol{\Sigma}_{\mathrm{i} / \mathrm{e}}$, Ohm's law provides the expression for the current density $\mathbf{J}_{\mathrm{i} / \mathrm{e}}$ by

\footnotetext{
${ }^{16}$ Actually these and other variables are position and time dependent, thus the exact notation is $V_{\mathrm{i}}(t, \mathbf{r})$, and $V_{\mathrm{e}}(t, \mathbf{r})$, respectively. However, for the sake of clarity we do not write this dependencies explicitly, if it is apparent from the context.
} 


$$
\begin{aligned}
& \mathbf{J}_{\mathrm{i}}=\boldsymbol{\Sigma}_{\mathrm{i}} \cdot \mathbf{E}_{\mathrm{i}}=-\boldsymbol{\Sigma}_{\mathrm{i}} \cdot \vec{\nabla} V_{\mathrm{i}}, \\
& \mathbf{J}_{\mathrm{e}}=\boldsymbol{\Sigma}_{\mathrm{e}} \cdot \mathbf{E}_{\mathrm{e}}=-\boldsymbol{\Sigma}_{\mathrm{e}} \cdot \vec{\nabla} V_{\mathrm{e}} .
\end{aligned}
$$

Since electrical charge is a conserved quantity, it can either propagate along the domain or in the transmembrane direction, that means towards the other domain. The continuity equation for the electrical charges including both domains reads therefore

$$
\vec{\nabla} \cdot \Sigma_{\mathrm{i}} \vec{\nabla} V_{\mathrm{i}}+\vec{\nabla} \cdot \boldsymbol{\Sigma}_{\mathrm{e}} \vec{\nabla} V_{\mathrm{e}}=0
$$

The physical meaning of Eq. 2.31) is, that if electrical charge leaves one domain, it enters the other domain and vice versa. This transmembrane current is effectively a change of the charge density of the domains $\frac{\partial q_{\mathrm{i}}}{\partial t}=\vec{\nabla} \cdot \boldsymbol{\Sigma}_{\mathrm{i}} \vec{\nabla} V_{\mathrm{i}}=-\vec{\nabla} \cdot \boldsymbol{\Sigma}_{\mathrm{e}} \vec{\nabla} V_{\mathrm{e}}=-\frac{\partial q_{\mathrm{e}}}{\partial t}$ and can be modeled by

$$
\frac{\partial q_{\mathrm{i}}}{\partial t}=\chi\left(C_{\mathrm{m}} \frac{\partial\left(V_{\mathrm{i}}-V_{\mathrm{e}}\right)}{\partial t}+I_{\text {Ion }}\right)
$$

where $\chi$ is the membrane surface to volume ratio of the tissue, $C_{\mathrm{m}}$ is the electrical capacitance of the membrane per surface area of the membrane and $I_{\text {Ion }}$ specifies the currents due to the ion channel dynamics.

Outside the cardiac tissue in the surrounding medium, one can also define a conductivity tensor $\boldsymbol{\Sigma}_{0}$ and since charge conservation is also valid here, the continuity equation for the surrounding medium reads

$$
\vec{\nabla} \cdot \Sigma_{0} \vec{\nabla} V_{0}=0
$$

Thus, the differential equations can be summed up by introducing the membrane potential $V_{\mathrm{m}}=V_{\mathrm{i}}-V_{\mathrm{e}}$ :

$$
\begin{array}{cc}
\vec{\nabla} \cdot \boldsymbol{\Sigma}_{\mathrm{i}} \vec{\nabla} V_{\mathrm{m}}+\vec{\nabla} \cdot\left(\boldsymbol{\Sigma}_{\mathrm{e}}+\boldsymbol{\Sigma}_{\mathrm{i}}\right) \vec{\nabla} V_{\mathrm{e}}=0 & \forall \mathbf{r} \in \mathbb{T}, \\
\vec{\nabla} \cdot \boldsymbol{\Sigma}_{\mathrm{i}} \vec{\nabla} V_{\mathrm{m}}+\vec{\nabla} \cdot \boldsymbol{\Sigma}_{\mathrm{i}} \vec{\nabla} V_{\mathrm{e}}=\chi\left(C_{\mathrm{m}} \frac{\partial V_{\mathrm{m}}}{\partial t}+I_{\text {Ion }}\right) & \forall \mathbf{r} \in \mathbb{T}, \\
\vec{\nabla} \cdot \boldsymbol{\Sigma}_{0} \vec{\nabla} V_{0}=0 & \forall \mathbf{r} \in \mathbb{M} .
\end{array}
$$

Note that Eqs. (2.34) - 2.35) hold inside the tissue $(\mathbb{T})$, whereas Eq. 2.36) describes the diffusive dynamics in the surrounding medium $(\mathbb{M})$. 
The system of Eqs. 2.34 - 2.36 can only be solved with boundary conditions. Let us first assume that the surrounding medium is electrically isolated from the outside $(\partial \mathbb{M})$. Furthermore, at the boundary between the tissue and the surrounding medium $(\partial \mathbb{T})$ the intracellular domain is not electrically connected to the medium, thus $\mathbf{n} \cdot \boldsymbol{\Sigma}_{\mathrm{i}} \vec{\nabla} V_{\mathrm{i}}=0$, where $\mathbf{n}$ is a normalized vector perpendicular to the boundary. However, the extracellular domain couples to the surrounding domain. Summarizing this (using $V_{\mathrm{m}}=V_{\mathrm{i}}-V_{\mathrm{e}}$ ) reads

$$
\begin{aligned}
\mathbf{n} \cdot \boldsymbol{\Sigma}_{0} \vec{\nabla} V_{0} & =0 & & \forall \mathbf{r} \in \partial \mathbb{M}, \\
\mathbf{n} \cdot \boldsymbol{\Sigma}_{\mathrm{i}} \vec{\nabla} V_{\mathrm{m}}+\mathbf{n} \cdot \boldsymbol{\Sigma}_{\mathrm{i}} \vec{\nabla} V_{\mathrm{e}} & =0 & & \forall \mathbf{r} \in \partial \mathbb{T} \\
\mathbf{n} \cdot \boldsymbol{\Sigma}_{0} \vec{\nabla} V_{0} & =\mathbf{n} \cdot \boldsymbol{\Sigma}_{\mathrm{e}} \vec{\nabla} V_{\mathrm{e}} & & \forall \mathbf{r} \in \partial \mathbb{T} \\
V_{\mathrm{e}} & =V_{0} & & \forall \mathbf{r} \in \partial \mathbb{T}
\end{aligned}
$$

Equations 2.37 - 2.40 together with the boundary conditions in Eqs. 2.34 - 2.36 provide a closed system of equations which can be solved.

\section{The Monodomain model}

The bidomain model describes the dynamics by considering two domains. Although this is an intuitive approach for describing the action potential propagation, in practice numerical simulations of this model are computationally demanding. In each simulation step, after solving Eq. 2.35), $V_{\mathrm{e}}$ needs to be determined by Eq. (2.34), which numerically results in inverting a matrix. This is already for a moderate size of the matrix (mainly determined by the size of the simulation domain) quite expensive. However, under certain conditions the bidomain equations can be simplified, which significantly reduces the computation time. In the case that the intra- and extracellular conductivities differ only by a constant factor $\boldsymbol{\Sigma}_{\mathrm{e}}=\eta \boldsymbol{\Sigma}_{\mathrm{i}}$ ("equal anisotropy ratio"), Eq. 2.34 can be rewritten to

$$
\frac{1}{1+\eta} \vec{\nabla} \cdot \Sigma_{\mathrm{i}} \vec{\nabla} V_{\mathrm{m}}=-\vec{\nabla} \cdot \boldsymbol{\Sigma}_{\mathrm{i}} \vec{\nabla} V_{\mathrm{e}}
$$

which can then be inserted in Eq. 2.35 in order get rid of $V_{\mathrm{e}}$ :

$$
\frac{\eta}{1+\eta} \vec{\nabla} \cdot \boldsymbol{\Sigma}_{\mathrm{i}} \vec{\nabla} V_{\mathrm{m}}=\chi\left(C_{\mathrm{m}} \frac{\partial V_{\mathrm{m}}}{\partial t}+I_{\text {Ion }}\right) \forall \mathbf{r} \in \mathbb{T}
$$

This can be rewritten, by introducing the diffusion tensor (rescaled conductivity) $\mathbf{D}=\frac{\eta}{1+\eta} \frac{1}{\chi C_{\mathrm{m}}} \boldsymbol{\Sigma}_{\mathrm{i}}$ to the typical form of a partial differential equation:

$$
\frac{\partial V_{\mathrm{m}}}{\partial t}=\vec{\nabla} \cdot \mathbf{D} \vec{\nabla} V_{\mathrm{m}}-\frac{I_{\text {Ion }}}{C_{\mathrm{m}}}
$$


By combining Eq. (2.38) and (2.39) using $\mathbf{n} \cdot \boldsymbol{\Sigma}_{\mathrm{e}} \vec{\nabla} V_{\mathrm{e}}=\mathbf{n} \cdot \eta \boldsymbol{\Sigma}_{\mathrm{i}} \vec{\nabla} V_{\mathrm{e}}$ the boundary condition for the monodomain approach can be formulated as $\mathbf{n} \cdot \eta \boldsymbol{\Sigma}_{\mathrm{i}} \vec{\nabla} V_{\mathrm{m}}=-\mathbf{n} \cdot \boldsymbol{\Sigma}_{0} \vec{\nabla} V_{0}$.

Thus, the membrane potential $V_{\mathrm{m}}$ couples at the boundary $\partial \mathbb{T}$ to the potential of the surrounding medium $V_{0}$. In this thesis however, we assume that the heart is basically decoupled from the surrounding medium. This common assumption reduces the complexity of the simulations, but is not true in the general case e.g. of a heart inside the body. However, numerical and experimental studies indicate [59, 60] that in the case of an isolated heart there are no significant differences in the excitation patterns inside the heart in comparison to a conducting boundary between the heart and the surrounding medium. That means, in practice in most cases the (realistic) property of a non-zero conduction through the outer boundary of the heart is mainly relevant in numerical studies which investigate the propagation of the electrical signal outside the heart (e.g. simulations with the objective to calculate a realistic electrocardiogram [61]). For all simulations in this thesis we assume $\mathbf{n} \cdot \boldsymbol{\Sigma}_{i} \vec{\nabla} V_{\mathrm{m}}=0$. However, for reasons of completeness, we mention at this point that in the case of the application of a high energy shock (an external electrical field), the gradient of the potential outside the medium is not negligible $-\vec{\nabla} V_{0}=\mathbf{E}_{\text {ext }}$ and the boundary effects play a major role [62, 63]:

$$
\mathbf{n} \cdot \underbrace{\eta \boldsymbol{\Sigma}_{\mathrm{i}}}_{=\boldsymbol{\Sigma}_{\mathrm{e}}} \vec{\nabla} V_{\mathrm{m}}=\mathbf{n} \cdot \boldsymbol{\Sigma}_{0} \mathbf{E}_{\text {ext }} .
$$

However, in the absence of an external electrical field $\mathbf{E}_{\text {ext }}$, Eq. 2.44) reduces to a no-flux boundary condition (which is used in all studies of this thesis).

Finally, the system of equations for the monodomain approximation reads:

$$
\begin{array}{rlrl}
\frac{\partial V_{\mathrm{m}}}{\partial t} & =\vec{\nabla} \cdot \mathbf{D} \vec{\nabla} V_{\mathrm{m}}-\frac{I_{\text {Ion }}}{C_{\mathrm{m}}} & & \forall \mathbf{r} \in \mathbb{T}, \\
\mathbf{n} \cdot \boldsymbol{\Sigma}_{\mathrm{e}} \vec{\nabla} V_{\mathrm{m}} & =0 & \forall \vec{r} \in \partial \mathbb{T} .
\end{array}
$$

\section{Validation of the "Equal Anisotropy Ratio" Assumption}

The monodomain equations Eqs. 2.45) - 2.46) in comparison to the bidomain equations Eqs. 2.37) - 2.40 provide a significant reduction of complexity and computational cost. Still, this simplification is associated with the assumption of an "equal anisotropy ratio" $\left(\boldsymbol{\Sigma}_{\mathrm{e}}=\eta \boldsymbol{\Sigma}_{\mathrm{i}}\right)$. We want to clarify, to which extent this assumption is valid, and therefore how accurate the monodomain approximation is in numerical simulations.

As discussed in section 2.1 .2 on page 17, the conductivity at a specific point inside the cardiac tissue is not isotropic but there exist distinct directions due to the local fiber orientation. That is, why in the most general case, conductivity in our equations has the mathematical form of a tensor. One distinguishes between three orthogonal directions and their conductivities: the longitudinal direction, aligned parallel to the fiber and along the 
Table 2.1: An overview of experimental measurements (comprising three studies) of the longitudinal and perpendicular conductivity $\sigma_{\ell}$ and $\sigma_{\mathrm{p}}$, respectively, in the extra- and intracellular domain of cardiac tissue. In addition, the ratios $\sigma_{\ell}^{\mathrm{e}} / \sigma_{\ell}^{\mathrm{i}}$ and $\sigma_{\mathrm{p}}^{\mathrm{e}} / \sigma_{\mathrm{p}}^{\mathrm{i}}$ are shown.

\begin{tabular}{ccccccc}
\hline Reference & $\begin{array}{c}\sigma_{\ell}^{\mathrm{i}} \\
{\left[\frac{\mathrm{S}}{\mathrm{m}}\right]}\end{array}$ & $\begin{array}{c}\sigma_{\mathrm{p}}^{\mathrm{i}} \\
{\left[\frac{\mathrm{S}}{\mathrm{m}}\right]}\end{array}$ & $\begin{array}{c}\sigma_{\ell}^{\mathrm{e}} \\
{\left[\frac{\mathrm{S}}{\mathrm{m}}\right]}\end{array}$ & $\begin{array}{c}\sigma_{\mathrm{p}}^{\mathrm{e}} \\
{\left[\frac{\mathrm{S}}{\mathrm{m}}\right]}\end{array}$ & $\sigma_{\ell}^{\mathrm{e}} / \sigma_{\ell}^{\mathrm{i}}$ & $\sigma_{\mathrm{p}}^{\mathrm{e}} / \sigma_{\mathrm{p}}^{\mathrm{i}}$ \\
\hline$[64]$ & 0.17 & 0.019 & 0.62 & 0.24 & 3.65 & 12.63 \\
{$[65]$} & 0.28 & 0.026 & 0.22 & 0.13 & 0.79 & 5.00 \\
{$[66]$} & 0.34 & 0.060 & 0.12 & 0.080 & 0.35 & 1.33 \\
\hline
\end{tabular}

fiber sheet (with the conductivity $\sigma_{\ell}$ ), the direction perpendicular to the fiber and still along the fiber sheet $\left(\sigma_{\mathrm{p}}\right)$, and the direction transversal to the first two directions, thus, orthogonal to the sheets of fiber $\left(\sigma_{\mathrm{t}}\right)$ (see for example 2.5 on page 18$)$. The conductivities in these directions are furthermore different for the intra- and extracellular domain (e.g. $\left.\sigma_{\ell}^{\mathrm{i} / \mathrm{e}}\right) .17$ Since these local conductivities eventually determine the specific form of the conductivity tensors $\boldsymbol{\Sigma}_{\mathrm{i} / \mathrm{e}}=\boldsymbol{\Sigma}_{\mathrm{i} / \mathrm{e}}\left(\sigma_{\mathrm{P}}^{\mathrm{i} / \mathrm{e}}, \sigma_{\mathrm{t}}^{\mathrm{i} / \mathrm{e}}, \sigma_{\ell}^{\mathrm{i} / \mathrm{e}}\right)$ in Eqs. 2.34 -2.35 , the assumption of an "equal anisotropy ratio" used for the derivation of the monodomain model can be broken down to the local conductivities.

Table 2.1 shows results of three studies, aiming at measuring the conductivities in the longitudinal direction, and perpendicular to the fiber along the fiber sheet direction [64, [65, 66. In addition, the ratios $\sigma_{\ell}^{\mathrm{i}} / \sigma_{\mathrm{p}}^{\mathrm{i}}$ and $\sigma_{\ell}^{\mathrm{e}} / \sigma_{\mathrm{p}}^{\mathrm{e}}$ are determined. These studies indicate, that a precise measurement of the conductivities is experimentally difficult and that the assumption of an "equal anisotropy ratio" can hardly be justified.

That is, why diverse studies investigated the qualitative differences of the bidomain and the monodomain model. They show, that the monodomain approximation has only negligible effects on quantities like the propagation velocities [67] or activation times [68]. However, in the case of the application of external electrical fields, differences in the shape of the resulting excitation patterns (induced by virtual electrodes, see section 2.1.5 on page 22 ) have been observed [69, 70]. The studies of this thesis do not rely on the realistic simulation of excitation patterns caused by electrical fields. That is, why the monodomain model is a good approximation and it is used for all simulations of this thesis.

\subsubsection{Models of Local Cell Dynamics}

Equations 2.34 - 2.36 and 2.45 - 2.46 are denoted as "reaction-diffusion equations", since they contain both, a diffusive part, and a local reaction part. In order to solve Eq. 2.45) or Eq. 2.35), the ion channel dynamics is still needed $\left(I_{\text {Ion }}\right)$, which results in additional differential equations. Here we discuss models which describe the local ion channel dynamics on a cellular level. As described in section 2.1.1 on page 11 the membrane potential $V_{\mathrm{m}}$ of a cell is composed of ion concentrations of different types (e.g. sodium, calcium or potassium). These concentrations change due to diverse mechanisms, like leak channels, the $\mathrm{Na}^{+} / \mathrm{K}^{+}$-ATPase or voltage gated ion channels.

\footnotetext{
${ }^{17}$ The conductivities are also spatially dependent, thus they are actually local variables $(\sigma=\sigma(\mathbf{r}))$.
} 
The models discussed here aim to describe these underlying mechanisms on a more or less detailed and realistic level (depending on the actual model), in order to reproduce the characteristic behavior of a single cell. Various features of the cell dynamics that shall be reproduced by the models are the upstroke, duration, and refractory period of an action potential, for example.

Sets of coupled differential equations are used for this purpose. From a mathematical point of view it turns out, that for modeling basic features like the refractory behavior more, at least two dynamical variables and two equations are necessary. That means, additionally to the diffusive Eqs. 2.35) or Eq. 2.45), respectively, which describe the local dynamics and the diffusive behavior of the membrane potential $V_{\mathrm{m}}$, at least one additional equation is needed, describing the evolution of at least one additional variable. The need for additional variables can be explained by the refractive property of the tissue which demands some kind of memory of the past. Accordingly, the simplest models which describe excitable media are two-dimensional (the membrane potential $V_{\mathrm{m}}$ and one additional variable).

Simple models which are commonly used to describe excitable media (also apart from cardiac tissue) are for example the Barkley model [71] (three parameters) or the Aliev-Panfilov model [72] (five parameters), which provide only basic features of excitable media, but are extremely fast to compute. However, more underlying equations and variables can be included into the models, in order to be more realistic and to reproduce more details of the dynamics of actual cardiac tissue. With this gain of realism and level of detail, also the computational cost increases. The class of "ionic models" comprises individual equations and variables for single ion channels and specific ion concentrations. The Luo-Rudy II model [73] (fifteen dynamical variables) is an example for such a rather detailed model. The choice of the proper cardiac cell model used for a numerical study or, more general, for the respective scientific objective is discussed in section 2.3 on page 40 . In the following, the Aliev-Panfilov model and the Fenton-Karma model [26] are presented as examples for a rather simple model and a model with moderate level of complexity, respectively, in order to elucidate the general form of the underlying equations.

\section{Aliev-Panfilov Model}

Equations (2.47)-(2.49) depict the Aliev-Panfilov model, here used in the context of the monodomain approach:

$$
\begin{aligned}
\frac{\partial V_{\mathrm{m}}}{\partial t} & =\nabla \cdot \underline{\mathbf{D}} \nabla V_{\mathrm{m}}-k V_{\mathrm{m}}\left(V_{\mathrm{m}}-1\right)\left(V_{\mathrm{m}}-a\right)-V_{\mathrm{m}} v \\
\frac{\partial v}{\partial t} & =\epsilon\left(V_{\mathrm{m}}, v\right)\left(-v-k V_{\mathrm{m}}\left(V_{\mathrm{m}}-a-1\right)\right) .
\end{aligned}
$$

Thus, Eq. 2.47) corresponds to Eq. (2.45), where the first term still describes the diffusion of the first membrane potential, but the local reaction part (second and third term) is now specifically given by the Aliev-Panfilov model. The evolution of the second variable $v$ is furthermore described in Eq. 2.48), and the expression for $\epsilon\left(V_{\mathrm{m}}, v\right)$ reads 


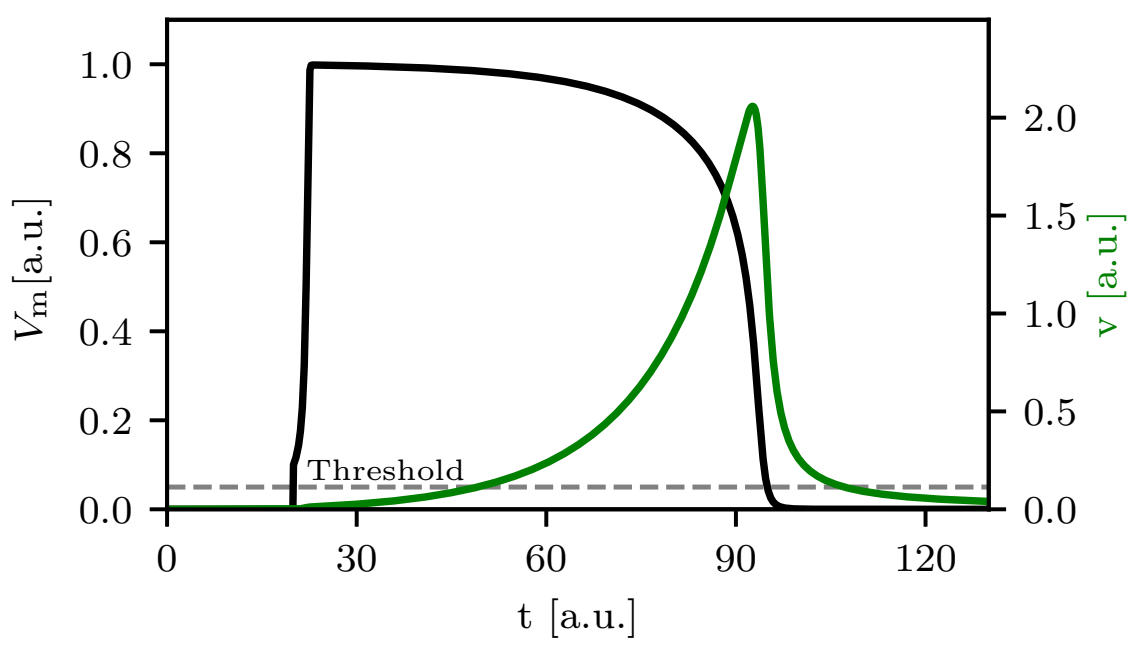

Figure 2.20: A representative action potential using the Aliev-Panfilov model, with the parameters $a=0.05, \epsilon_{0}=0.002, \mu_{1}=0.2, \mu_{2}=0.3$ and $k=8$. The membrane potential $V_{\mathrm{m}}$ (black) and the second dynamical variable $v$ (green) are shown. The action potential was triggered by an external increase of the membrane potential from zero to $V_{\mathrm{m}}=0.1 \mathrm{a}$.u. at $t=20$ a.u. exceeding the excitation threshold $(a=0.05)$.

$$
\epsilon\left(V_{\mathrm{m}}, v\right)=\epsilon_{0}+\frac{\mu_{1} v}{V_{\mathrm{m}}+\mu_{2}}
$$

These simple models have also the advantage (beyond their fast computation time), that some of the parameters have a direct physical meaning. In this case, parameter $a$ is the excitation threshold and parameter $k$ determines the excitability (excitation threshold) of the cell (see section 2.1.1 on page 11). However, the other parameters $\mu_{1}, \mu_{2}$ and $\epsilon_{0}$ are used to fit the shape of the cardiac action potential. In Fig. 2.20 a representative action potential is shown, with both dynamical variables $V_{\mathrm{m}}$ and $v 18$

\section{Fenton-Karma Model}

The Fenton-Karma model (or 3V-model) [26] is a cardiac cell model with a moderate level of complexity (three dynamical variables and thirteen parameters) but still offers a reasonable computational demand. It describes the transmembrane current $I_{\text {Ion }}$ in Eq. 2.45) by three distinct current densities: the fast inward current $I_{\mathrm{fi}}$, the slow outward current $I_{\mathrm{so}}$ and the slow inward current $I_{\mathrm{si}}$ in Eq. 2.53):

\footnotetext{
${ }^{18}$ This action potential is already computed numerically. Details about the numerical algorithms used to solve the underlying differential equations will be given later.
} 


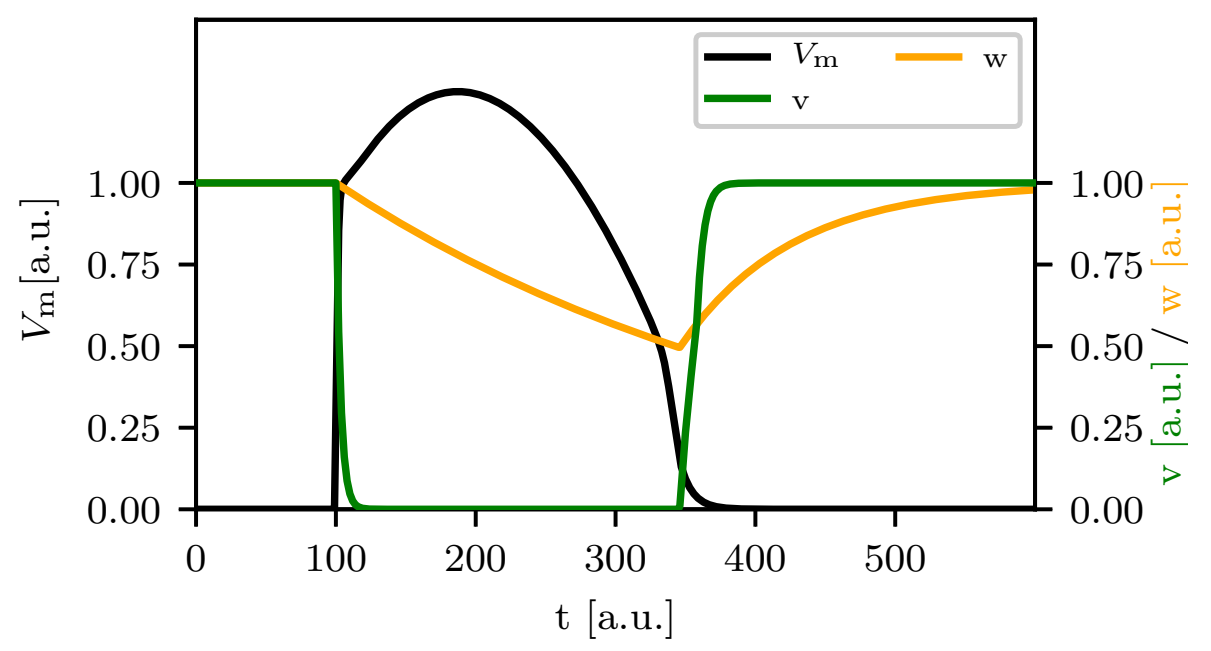

Figure 2.21: A representative action potential using the Fenton-Karma model, with the the parameter set FK1 (see A.2 in the Appendix A on page 131). The membrane potential $V_{\mathrm{m}}$ (black) and the two other dynamical variables $v$ (green) and $w$ (orange) are shown. The action potential was triggered by an external increase of the membrane potential from zero to $V_{\mathrm{m}}=0.1$ a.u. at $t=100$ a.u..

$$
\begin{aligned}
I_{\mathrm{fi}}\left(V_{\mathrm{m}}, v\right) & =-\frac{v}{\tau_{\mathrm{d}}} \Theta\left(V_{\mathrm{m}}-u_{\mathrm{c}}\right)\left(1-V_{\mathrm{m}}\right)\left(V_{\mathrm{m}}-u_{\mathrm{c}}\right), \\
I_{\mathrm{so}}\left(V_{\mathrm{m}}\right) & =\frac{V_{\mathrm{m}}}{\tau_{0}} \Theta\left(u_{\mathrm{c}}-V_{\mathrm{m}}\right)+\frac{1}{\tau_{\mathrm{r}}} \Theta\left(V_{\mathrm{m}}-u_{\mathrm{c}}\right), \\
I_{\mathrm{si}}\left(V_{\mathrm{m}}, w\right) & =-\frac{w}{2 \tau_{\mathrm{si}}}\left(1+\tanh \left[k\left(V_{\mathrm{m}}-u_{c}^{\mathrm{si}}\right)\right]\right) .
\end{aligned}
$$

These currents aim to model the most prevalent dynamics of sodium, potassium and calcium (discussed in section 2.1.1 on page 11) and are explicitly given in Eqs. (2.50)-(2.52). The evolution of two additional dynamical variables $(v, w)$ is shown in Eqs. 2.54)-2.55):

$$
\begin{aligned}
\frac{\partial V_{\mathrm{m}}}{\partial t} & =\nabla \cdot \underline{\mathbf{D}} \nabla V_{\mathrm{m}}+\left[I_{\mathrm{fi}}\left(V_{\mathrm{m}}, v\right)-I_{\mathrm{so}}\left(V_{\mathrm{m}}\right)-I_{\mathrm{si}}\left(V_{\mathrm{m}}, w\right)\right] / C_{\mathrm{m}}, \\
\frac{\partial v}{\partial t} & =\Theta\left(u_{\mathrm{c}}-V_{\mathrm{m}}\right)(1-v)\left(\frac{\Theta\left(V_{\mathrm{m}}-u_{\mathrm{v}}\right)}{\tau_{\mathrm{v} 1}^{-}}+\frac{\Theta\left(u_{\mathrm{v}}-V_{\mathrm{m}}\right)}{\tau_{\mathrm{v} 2}^{-}}\right)-\Theta\left(V_{\mathrm{m}}-u_{\mathrm{c}}\right) \frac{v}{\tau_{\mathrm{v}}^{+}}, \\
\frac{\partial w}{\partial t} & =\Theta\left(u_{\mathrm{c}}-V_{\mathrm{m}}\right) \frac{1-w}{\tau_{\mathrm{w}}^{-}}-\Theta\left(V_{\mathrm{m}}-u_{\mathrm{c}}\right) \frac{w}{\tau_{\mathrm{w}}^{+}} .
\end{aligned}
$$

In Fig. 2.21, a representative action potential is shown, with all three dynamical variables $\left(V_{m}, v\right.$, and $\left.w\right)$.

All sets of parameters, and further cardiac cell models used in this thesis are presented in the Appendix A on page 131. 


\section{Impact of the Choice of Parameters}

Every cardiac cell models has a specific set of parameters which needs to be chosen before a simulation. It is important to understand, that the choice of parameters determines the local dynamics of the cell, thus the shape and properties of an action potential, but beyond also has a huge impact onto the dynamical behavior of spiral or scroll waves. The break-up mechanism, propagation behavior and filament tension of scroll waves [26] are for example important features, which are mainly determined by the local parameters. Fenton et al., for example, performed for that reason a single study only to investigate this dependence by testing ten different parameter sets for the Fenton-Karma model [74].

With properties or rules which are defined only locally but determine the dynamics on a global scale, this feature on the one hand illustrates in an interesting way the nature of complex systems. However, the sensitive dependence of parameters stresses also their significance concerning the design of proper numerical simulations which aim to address a specific scientific objective. The Fenton-Karma model combines a reasonable computation time, with a moderate level of complexity (thirteen parameters), which allows to reproduce diverse dynamics of spiral/scroll waves. That is, why this model was used for many studies of this thesis. More details about the choice of the cardiac cell model and its parameters are given in section 2.3 on page 40 .

\subsubsection{Discretization and Stability}

In the previous sections we derived and discussed the underlying systems of equations which we use to model cardiac tissue. In general, no analytical solutions are known for these equations. At this level of complexity, currently the only way to investigate the dynamics of these equations (on a non-trivial spatio-temporal level) is to use concepts from numerical mathematics. Thus, we need to discretize the equations (in space and time) and then determine the dynamical variables of the equations at these discrete points. In the following we present the actual discretization in space and time and discuss the related question of stability and accuracy of the numerical solution. We use here the finite-differences method FDM, in order to approximate the actual equations with difference equations. As an example of application, we discuss the monodomain equation Eq. 2.45), since this is also the most relevant equation in this thesis

$$
\frac{\partial V_{\mathrm{m}}}{\partial t}=\vec{\nabla} \cdot \underline{\mathbf{D}} \vec{\nabla} V_{\mathrm{m}}-\frac{I_{\mathrm{Ion}}\left(V_{\mathrm{m}}, \boldsymbol{v}\right)}{C_{\mathrm{m}}},
$$

where we assume that the local reaction term $I_{\text {Ion }}$ depends on the membrane potential $V_{\mathrm{m}}$ and a (not specified) number of secondary variables $\boldsymbol{v}$ (see section 2.4.2 on page 48).

\section{Discretization in Time}

As stated before, a general analytical solution for Eq. 2.56) is not known. For this reason, we solved the equation in this thesis using numerical algorithms, specifically a first order Runge- 
Kutta method, also known as explicit Euler method [75]. It is the simplest approach to solve a differential equation numerically, but, however, is not very accurate or stable in comparison to other more sophisticated methods. Still, this method is used in the studies of this thesis, since a significant property of valid numerical results concerning their applicability to real cardiac dynamics is their robustness to small parameter changes. In particular, results should not depend sensitively on the accuracy of the solutions of the underlying equations. More details about the role of numerical simulations are given in section 2.3 on page 40.

As a first step, we want to discuss the principles of this method considering a single point in space. The correlation and interaction between different locations in the full spatiotemporal space will be discussed in the next section. The explicit Euler method makes use of a Taylor expansion, in order to determine the evolution of a dynamical variable. Assuming an exemplary differential equation

$$
\frac{\partial x}{\partial t}=f(x, y)
$$

We want to know the evolution of the dynamical variable $x$ from time $t_{0}$ (thus $x\left(t_{0}\right)$ is known) to time $t_{0}+d t$, with the temporal step $d t$. Performing a Taylor expansion of $x$ at time $t_{0}$ and using $d t$ as the expansion parameter gives

$$
x\left(t_{0}+d t\right)=x\left(t_{0}\right)+d t \frac{\partial x}{\partial t}+d t^{2} \frac{\partial^{2} x}{\partial t^{2}}+\mathcal{O}\left(d t^{3}\right) .
$$

By neglecting terms of the order $\mathcal{O}\left(d t^{2}\right)$ and recognizing the first derivative $\frac{\partial x}{\partial t}$ Eq. 2.58 which is already given by Eq. 2.57), one calculation step can be formulated as

$$
x\left(t_{0}+d t\right) \approx x\left(t_{0}\right)+d t f(x, y) .
$$

For the monodomain equation Eq. (2.56), this means

$$
V_{\mathrm{m}}\left(t_{0}+d t\right) \approx V_{\mathrm{m}}\left(t_{0}\right)+d t\left[\vec{\nabla} \cdot \overrightarrow{\vec{D}} \vec{\nabla} V_{\mathrm{m}}\left(t_{0}\right)-\frac{I_{\text {Ion }}\left(V_{\mathrm{m}}\left(t_{0}\right), \vec{v}\left(t_{0}\right)\right)}{C_{\mathrm{m}}}\right]
$$

From Eq. 2.58) one can read that the error made in a single step (also called local truncation error $E_{\text {Local }}$ ) is approximately proportional to $d t^{2}$ (for small $d t$ ). It can be shown, that the global truncation error $E_{\text {Global }}$ (the error made when performing the evolution from $t_{0}$ to $t$ with a certain number of steps made, thus an accumulated error) grows approximately with $E_{\text {Global }} \sim d t \cdot C \cdot\left(e^{L\left(t-t_{0}\right)}-1\right)$, where $L$ and $C$ are constants [76]. 
(a)

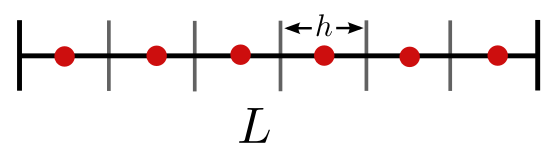

(b)

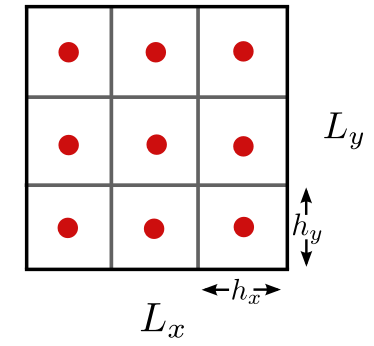

(c)

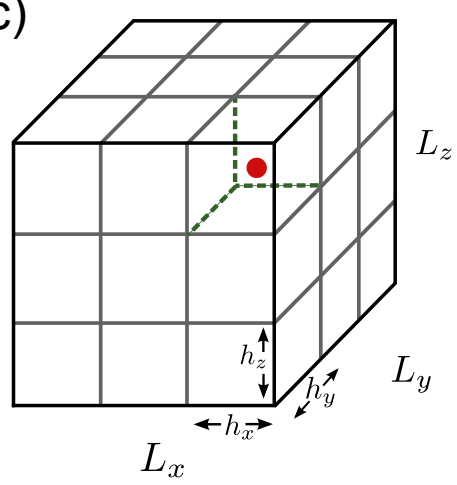

Figure 2.22: Discretization grids in different dimensions. A linear domain with length $L$ is shown in subplot (a), subdivided in segments of length $h$ (spacing constant). The grid nodes (red dots) represent the respective cell, and the dynamical variables of the underlying equations are solved representatively at these nodes. In two and three dimensions (subplots (b) and (c)) the corresponding objects are two-dimensional cells and three-dimensional cubes, respectively. In general, the spacing constants in different directions $\left(h_{x}, h_{y}, h_{z}\right)$ can have individual values. The length of the domain in each direction is given by $L_{i}=N_{i} \cdot h_{i}$, where $N_{i}$ is the number of grid nodes.

\section{Discretization in Space}

The underlying equations discussed in the previous sections can be used for numerical simulations of a point (0-dimensional), linear regimes (one-dimensional) and two- or threedimensional simulation domains. In all cases, except the first one, a spatially discretization (additionally to the discrete time) of the simulation domain is needed. Thus, the space where the simulations shall be performed is reduced to a (in our case regular) grid, with fixed grid points. By this procedure, linear segments (one-dimensional), cells (two-dimensional) or cubes (three-dimensional) are defined, with a corresponding grid point (Fig. 2.22).

The grid points represent in this approach the whole segment/cell/cube, and dynamical variables (e.g. $V_{\mathrm{m}}$ ) are defined only at the grid points, representing a spatially averaged value. Crucial for the subsequent calculations of the differential equations on a designed grid is the choice of the spacing constant $h$ (or $h_{x}, h_{y}$ and $h_{z}$, respectively for different directions). The multiplication of the number of grid nodes in a specific direction $N_{i}$ with the spacing constant yields the size of the simulation domain $L_{i}=N_{i} \cdot h_{i}$.

From a computational point of view, the essential question is where the spatial discretization of the simulation domain enters the governing differential equations. First of all, as already mentioned before, the equations are not solved at all spatial positions of the simulation domain, but just at specific points, the grid nodes. Furthermore, the local reaction part of the equations (second term in Eq. (2.56) ) and also the underlying equations concerning the cell dynamics of the simulation (2.4.2 on page 48 ) are solved only locally at the respective grid points. The diffusive part of the monodomain equation (first term in Eq. (2.56), however, comprises the interaction between different positions in space (and thus between different grid points). In all simulations of this thesis, a constant diffusion $(\underline{\mathbf{D}}=D)$ is 
assumed. Thus, the diffusion term (first term on the right hand side of Eq. 2.56) which needs to be discretized simplifies to the Laplace operator:

$$
\vec{\nabla} \cdot \underline{\mathrm{D}} \vec{\nabla} V_{\mathrm{m}}=D \Delta V_{\mathrm{m}}
$$

In one dimension, a simple discretization of Eq. (2.61) can be found intuitively using the central differences method. The diffusive contribution for the membrane potential at the grid point coordinate denoted with $x$ yields then $\Delta V_{\mathrm{m}}^{x} \approx \frac{V_{\mathrm{m}}^{x-1}-2 V_{\mathrm{m}}^{x}+V_{\mathrm{m}}^{x+1}}{h_{x}^{2}}$. In the more general case of two- and three dimensions, also other neighboring grid points contribute to the diffusive term of $V_{\mathrm{m}}^{x, y, z}$, with specific weighting coefficients. Thus, by including the next neighbors, the (discretized) approximation of the diffusive term can be formulated (assuming equal spacing constants in each direction $\left(h_{x}=h_{y}=h_{z}\right)$ ) using a coefficient matrix, or kernel $\alpha_{i, j, k}[77]$ :

$$
D \Delta V_{\mathrm{m}}^{x, y, z} \approx \frac{D}{h^{2}} \sum_{i, j, k \in\{-1,0,1\}} \alpha_{i, j, k} V_{\mathrm{m}}^{x+i, y+j, z+k} .
$$

A straightforward generalization of the one dimensional case using the central differences method to three dimensions yields the coefficients:

$$
\alpha_{i, j, k}=\frac{D}{h^{2}} \cdot\left\{\begin{array}{lll}
-6 & \text { for } & i=j=k=0 \\
1 & \text { for } & |i|+|j|+|k|=1 \\
0 & \text { for } & |i|+|j|+|k|=2
\end{array}\right.
$$

which can be transformed to two dimensions by a summation over the third dimension $\alpha_{i, j}^{2 D}=\sum_{k} \alpha_{i, j, k}$. This can be presented in a matrix form, where the coefficients of the matrix $\alpha_{i, j}^{2 D}$ run from -1 to 1 :

$$
\alpha_{i, j}^{2 D}=\frac{D}{h^{2}}\left(\begin{array}{ccc}
0 & 1 & 0 \\
1 & -4 & 1 \\
.0 & 1 & 0
\end{array}\right)
$$

$\alpha_{i, j}^{2 D}$ is commonly known as the "five-point stencil", due to five contributing points. It can be shown, that the error of this approximation is of the order of $\mathcal{O}\left(h^{2}\right)$. Taking more neighboring points into account, another kernel can be formulated as: 


$$
\tilde{\alpha}_{i, j, k}=\frac{D}{30 h^{2}} \cdot\left\{\begin{array}{lll}
-128 & \text { for } & i=j=k=0 \\
14 & \text { for } & |i|+|j|+|k|=1 \\
3 & \text { for } & |i|+|j|+|k|=2 \\
1 & \text { for } & |i|+|j|+|k|=3
\end{array} .\right.
$$

In comparison to $\alpha_{i, j, k}$ (Eq. (2.63)), the error of the approximation using the more complex "27-point stencil" $\tilde{\alpha}_{i, j, k}$ is also of the order of $\mathcal{O}\left(h^{2}\right)$. However, its rotational symmetry is more accurate than $\alpha_{i, j, k}$, and in practice concerning actual numerical simulations of excitation patterns on a regular grid, this results in less numerical distortions related to the rectangular topology of the grid nodes [77].

The two-dimensional representation can be obtained by the same procedure as above, and is denoted as the "nine-point stencil" [78]:

$$
\widetilde{\alpha}_{i, j}^{2 D}=\frac{D}{6 h^{2}}\left(\begin{array}{ccc}
1 & 4 & 1 \\
4 & -20 & 4 \\
1 & 4 & 1
\end{array}\right)
$$

In all numerical simulations of excitable media in this thesis, the "nine-point stencil" (in twodimensional simulations) and the "27-point stencil" (in three-dimensional simulations) were used to model the diffusive part of the reaction-diffusion equations. Only in one dimensional simulations of specific non-cardiac models (section 3.2 on page 76 ), the central difference approximation (linear example from the beginning of this section) is used.

\section{Accuracy and Stability of Equations}

For actual simulations, discretization parameters need to be determined (in particular the time stepping parameter $d t$ and the spacing constant $h$ ). The choice of these parameters is crucial for the stability, accuracy and the computation time. In detail, a small value for $d t$ will (in most cases) increase the accuracy of the numerical solution of the differential equations, while a larger value reduces the computation time (smaller number of computation steps for the same amount of simulated time), but is less accurate and if $d t$ is too large the numerical solution of the underlying equations will not be stable, and thus will give unphysical values (e.g. diverge). This provides a natural upper limit for the choice of $d t$. Similarly, a small spacing constant $h$ increases the spatial resolution, and thus the spatial accuracy. However, reducing the value of $h$ increases the number of grid points when the size of the simulation domain should be kept constant $\left(L_{x}=h \cdot N_{x}\right)$, and that means the same simulation takes a longer computation time (due to the increased number of grid points).

In practice, a reasonable compromise between accuracy and computation time has to be 
found. In addition, stability of the numerical solution needs to be ensured. From a physical point of view, the problem itself (that means the topology of the simulated dynamics), specifies reasonable numbers or limits for $d t$ and $h$ : The choice of the discretization parameters must enable a sufficient resolution of the central object: the action potential. Specifically, the rapid upstroke of the action potential needs to be resolved adequately (thus, $d t$ has to be smaller than the typical time scale of the upstroke $d t \leq \tau_{\text {upstroke }}$ ) [77. In addition, instabilities can also emerge from the diffusive dynamics in the underlying equations. A "von Neumann stability analysis" of the discretized version of the Laplace operator using Eq. 2.63) provides the stability condition [77]:

$$
D \frac{d t}{h^{2}} \leq \frac{1}{d}
$$

where $d$ is the dimension and $D$ is the diffusion constant.

\subsubsection{Boundary Conditions}

In section 2.4 .1 on page 44 the underlying equations were derived, including boundary conditions for the boundaries between the cardiac tissue and an outer medium (e.g. a bath). In the absence of an external electrical field, a Neumann boundary condition (noflux) was derived for the membrane potential $V_{\mathrm{m}}$ (Eq. (2.46)). Since secondary variables of the ionic cell model are not diffusive (they are only local), the boundary condition only needs to be specified for the membrane potential. In the following, we present how the no-flux boundary condition for the membrane potential is implemented for two cases. In the first part we discuss how it is defined for the outer boundary of the simulation grid, which is used when the simulated cardiac tissue occupies the whole simulation domain. Furthermore, when we consider cardiac tissue of a non-trivial geometry embedded into a bath, we use the so called "phase field method", in order to establish the no-flux boundary condition between the arbitrarily shaped cardiac tissue and the surrounding bath. While numerical simulations of arbitrarily shaped simulation domains can be solved in a more straightforward way with "finite element methods" (FEM), the implementation demands a more sophisticated approach in the case of finite differences. The phase field method is shown in the second part.

\section{Outer Boundaries of the simulation grid}

The calculation of the diffusive part, using e.g. the nine-point stencil (Eq. (2.66) ) from the previous section, can not be performed in the conventional way at the grid nodes at the edges of the grid, since no neighboring points are available here which are required for the application of the stencil. Here, additional information need to be included, which specify the type of boundary conditions. For the desired no-flux boundary condition required for the membrane potential $V_{\mathrm{m}}$, so called "ghost points" are used, which are (imaginary) added at the edges of the grid. Figure 2.23 depicts this technique for the one-dimensional case. 


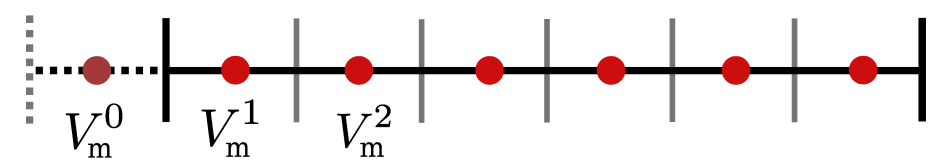

Figure 2.23: The use of "ghost points" in the context of the numerical realization of noflux boundary conditions. In the (exemplary) one-dimensional case, a ghost point with a new grid node is added at the edge of the grid. The virtual membrane potential of this node is denoted by $V_{\mathrm{m}}^{0}$. In the case of a no-flux boundary condition $V_{\mathrm{m}}^{0}$ takes the value of $V_{\mathrm{m}}^{2}$, in order to preserve a derivative of zero at $V_{\mathrm{m}}^{1}$.

For the calculation of the diffusive part of the underlying equations for the membrane potential at the edge of the grid, denoted by $V_{\mathrm{m}}^{1}$, a ghost point is added, which comprises an additional grid node with the virtual membrane potential at this point $V_{\mathrm{m}}^{0}$. In case of the no-flux boundary condition, the value of $V_{\mathrm{m}}^{0}$ is obtained by mirroring $V_{\mathrm{m}}$ along the edge, thus, $V_{\mathrm{m}}^{0}=V_{\mathrm{m}}^{2}$. In this way, the no-flux boundary condition can be achieved by a derivative of zero at the grid point $V_{\mathrm{m}}^{1}$. Thus, at the edges of the simulation grid the general expression for the diffusive term in one dimension $\Delta V_{\mathrm{m}}^{x} \approx \frac{V_{\mathrm{m}}^{x-1}-2 V_{\mathrm{m}}^{x}+V_{\mathrm{m}}^{x+1}}{h_{x}^{2}}$ reduces to

$$
\Delta V_{\mathrm{m}}^{1} \approx \frac{V_{\mathrm{m}}^{0}-2 V_{\mathrm{m}}^{1}+V_{\mathrm{m}}^{2}}{h_{x}^{2}}=\frac{2 V_{\mathrm{m}}^{2}-2 V_{\mathrm{m}}^{1}}{h_{x}^{2}}
$$

The subsequent generalization of this technique to two or three dimensional simulation domains is straightforward.

\section{Inner Boundaries Using the Phase Field Method}

When simulating arbitrarily shaped pieces of cardiac tissue (e.g. a whole heart in a surrounding bath), the use of ghost points is not feasible, due to the complex shape of the boundary. For this objective, the use of the "Phase Field method" is a very elegant way, to implement no-flux boundary conditions while keeping the finite difference scheme. The central object of this method is a phase field $\phi(\mathbf{r})$, which is defined at every grid node of the simulation domain, and has values between zero and one. In the simulations of this thesis, the phase field distinguishes between cardiac tissue $(\phi=1)$ and the surrounding bath $(\phi=0)$, and interpolates smoothly the boundaries between the two domains, with a certain transition zone with the width $\xi$. X. Li et al. showed, that if this phase field is included into the differential equations in a specific way, the desired no-flux boundary condition is automatically (approximately) fulfilled [79]. In the simulations performed in this thesis, the phase field enters the monodomain equation in the following way:

$$
\phi \frac{\partial V_{\mathrm{m}}}{\partial t}=\vec{\nabla} \cdot \underline{\mathbf{D}} \phi \vec{\nabla} V_{\mathrm{m}}-\phi \frac{I_{\text {Ion }}}{C_{\mathrm{m}}} .
$$

Flavio H. Fenton et al. demonstrated how this approach can be used for modeling electri- 

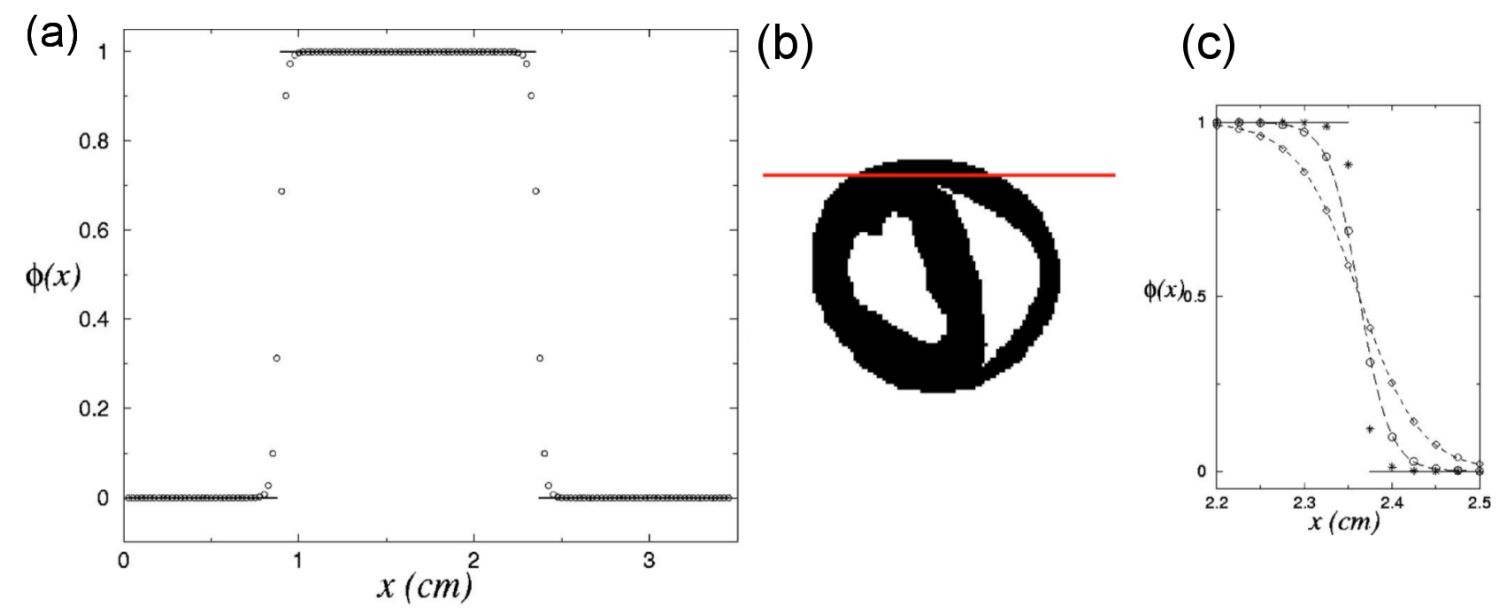

Figure 2.24: The Phase Field method. Subplot(a) shows an exemplary phase field along a cross section of a realistic heart geometry shown in (b). The section of the phase field shown in (a), corresponds to a cross section through the ventricular wall, marked by a red line in (b). Subplot (c) explains how the width of the transition zone between cardiac tissue and bath can be determined by the choice of the $\xi$ parameter $(\xi=0.075$ (diamonds), $\xi=0.05$ (circles) and $\xi=0.075$ (stars), respectively). Reprinted from [80], with the permission of AIP Publishing.

cal wave propagation in realistic heart geometries [80] and used the following differential equation for the creation of the phase field:

$$
\begin{aligned}
\frac{\partial \phi}{\partial t} & =\xi^{2} \Delta \phi-\frac{\partial G(\phi)}{\partial \phi} \\
G(\phi) & =-\frac{(2 \phi-1)^{2}}{4}+\frac{(2 \phi-1)^{4}}{8} .
\end{aligned}
$$

Figure 2.24 shows an example of how the phase field method is applied using a realistic heart geometry. The phase field is shown in subplot (a), where the smoothing procedure (solving Eq. (2.70) has already been performed. The representative section shown in (a) is marked by a red line in (b), which shows a cross section of the heart geometry, with the left and right ventricle. That means, the value around zero at $x=0 \mathrm{~cm}$ is located outside the tissue. When passing the epicardium, the phase field increases smoothly to one, before afterwards going back to zero, when leaving the ventricular wall. Subplot (c) shows a magnification of the transition zone. The phase field is plotted here using different values for $\xi$, which clarifies how the width of the transition zone can be modulated with the choice of $\xi$.

In practice, the phase field $\phi$ is initialized with the value zero (surrounding bath) and one (cardiac tissue). Using this initial field, Eq. 2.70) is solved numerically, (with the diffusive term modeled as described in the previous section). In the study of this thesis, where the phase field method was used (section 3.3 on page 102, Eq. 2.69) was solved with the parameters $d t=0.1, h=0.5$ and $\xi=0.5$. 


\subsubsection{Analyzing Tools}

The data obtained from numerical simulations in this thesis is in general high-dimensional and exhibits in most cases complex spatio-temporal patterns. In each single study which is presented in this thesis, the data needed to be analyzed concerning the respective scientific objective of the study. For this purpose, tools from various fields were used (e.g. nonlinear dynamics) that extracted relevant quantities and in this way enabled the interpretation of the results. In this section, the methods and their numerical implementation are presented.

\section{Determination of Phase Singularities}

Spiral waves (in two dimensions) and scroll waves (three dimensions), are the central objects which determine the spatio-temporal dynamics of excitation patterns in cardiac tissue. A central quantity used to describes the dynamics is the overall number of those spiral waves and scroll waves. With the method presented here, it is possible based on data of the membrane potential $V_{m}$ to identify the tips of the spiral waves, that means the organizing centers, using a mathematical procedure. In general, the spiral tips correspond to so called phase singularities in the phase representation of the data. Since the number of spiral tips corresponds one to one to the actual number of spiral waves, this method can be used to measure the total number of rotors in a system.

In order to analyze huge amounts of numerical (and also experimental) data, a two step protocol is commonly used, which exploits the periodic excitation dynamics of the tissue (the cycle of an action potential) [81, 82. In a first step, one can associate a phase to the (one dimensional) time series of the membrane potential $x(t)$ extracted from each single point of the simulation domain (or e.g. video data of experimental results, showing excitation patterns) [83]. This can be performed using different methods e.g. a delay embedding. In the studies of this thesis we used the Hilbert transformation $H\{x(t)\}$ [84]:

$$
H\{x\}(\tau)=\frac{1}{\pi} \int_{-\infty}^{\infty} \frac{x(t)}{\tau-t} d t=A(\tau) e^{i \theta(\tau)},
$$

where the phase $\theta$ can be extracted from the complex result of the Hilbert transformation. Figure 2.25 depicts the general functional principle of this method. A representative episode of two-dimensional chaotic dynamics in excitable media (simulation data taken from the study in section 3.1 on page 68 is shown in (a). An exemplary time series (of the grid point with the coordinates $(x, y)=(50,50))$ of the membrane potential is shown in subplot (c), along with the phase $\theta$ extracted using the Hilbert transform. This analysis was done for all grid points, resulting in the phase representation shown in subplot (b).

In a second step, phase singularities are determined at each point in time. The phase singularities correspond to the tips of the spiral waves and can be identified by a loop integral: 
(a)

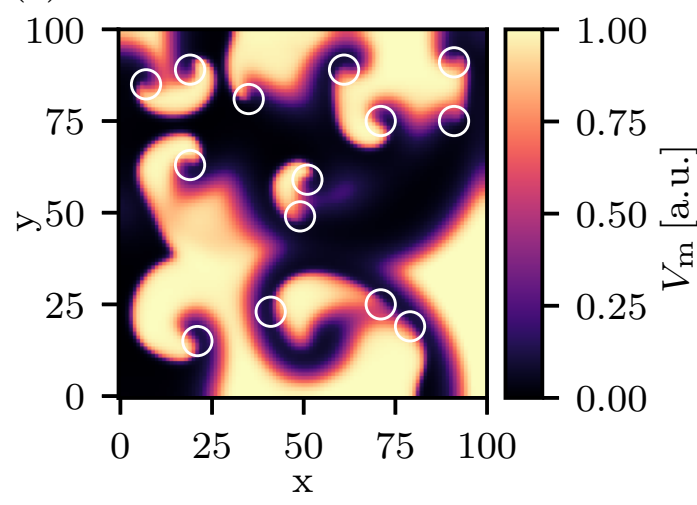

(b)

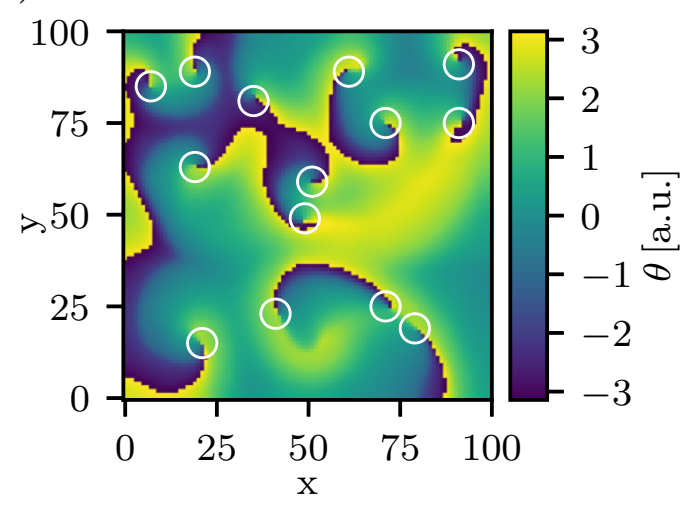

(c)

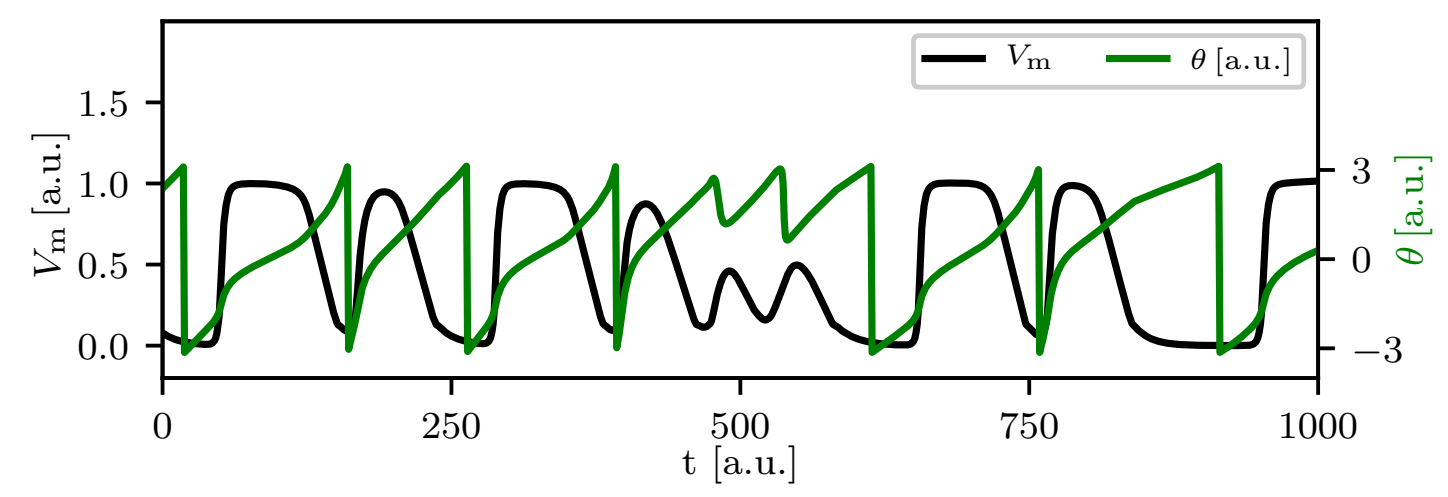

Figure 2.25: Determination of phase singularities using the Hilbert transformation. Subplot (a) shows a snapshot of the excitation pattern of spatio-temporal chaos in a twodimensional simulation using the Fenton-Karma model. The computed phase of the same snapshot is depicted in (b), where in both subplots white circles indicate the position of phase singularities. In subplot (c), the membrane potential $V_{\mathrm{m}}$ (black) and the corresponding phase $\theta$ (green) is plotted for the whole episode, for a representative grid node with the coordinates $(x, y)=(50,50)$ of the example shown in (a) and (b).

$$
n_{\mathrm{t}}=\frac{1}{2 \pi} \oint_{C} \vec{\nabla} \theta \cdot d \vec{l}
$$

If no phase singularity is located inside the integration zone $C$, the integral will yield zero. Otherwise, it provides the number of topological charges $n_{\mathrm{t}}$ (defined in [85, 86]), thus the sign of the charge is determined by the rotational direction of the spiral wave. Accordingly, in practice the integration zone of the line integral needs to be sufficiently small, to ensure that only one spiral tip fits into the integration zone. With this method, phase singularities (and that means spiral tips) can be identified, which are marked in Fig. 2.25(a) and (b) by white circles. 
(a)

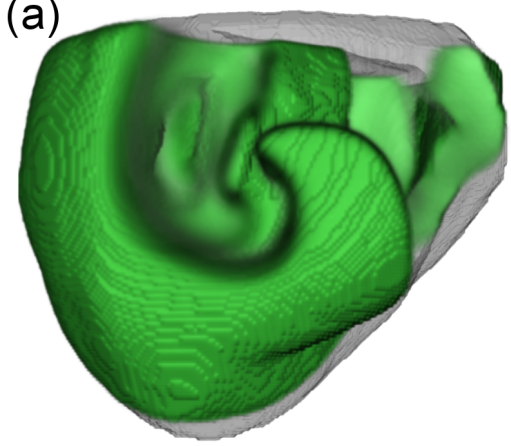

(b)

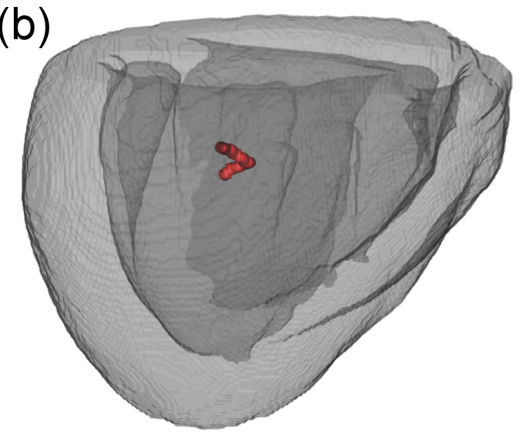

Figure 2.26: Reduction of a scroll wave to its filament. In subplot (a) the excitation pattern of a scroll wave is shown in green, whereas the corresponding filament (organizing center of the scroll wave) is depicted in (b) (red).

\section{Determination of Filaments}

The corresponding object to a two-dimensional spiral wave is a scroll wave in three dimensions, which can be thought of stacked spiral waves [26]. The zero dimensional spiral tip extends to a one dimensional curve, which is called the filament of the scroll wave. Similarly to the tips of a spiral wave, the number of scroll waves present in a system can be determined by the number of filaments. Furthermore, the usually very high-dimensional dynamics can be significantly reduced by the consideration of the filaments. Figure 2.26 shows the reduction of a scroll wave (a) to its filament (b). In particular in the study of section 3.3 on page 102 , the number of filaments is used as a measure for the complexity of the spatio-temporal state of the system.

Since filaments are the three-dimensional generalization of spiral tips (or phase singularities), the determination in numerical data is based on the concepts for the calculation of a phase singularity (previous section). At first, phase singularities are detected considering each of the three dimensions of the simulation domain separately. In a subsequent step, close phase singularities are assigned to the same filament. The assumption here is, that phase singularities from other filaments do not come closer then neighboring phase singularities of the same filament. The local cell dynamics (determines the propagation of scroll waves), the spacing constant $h$ and other parameters have an impact onto the validity of this assumption and need to be taken into account before a study.

\section{Calculation of Lyapunov Exponents}

The theoretical background of the computation of Lyapunov exponents was discussed in section 2.2.4 on page 34 Here, the numerical implementation of the algorithm proposed by Benettin et al. [46] is presented, which is used in studies of this thesis in order to determine Lyapunov exponents in extended (high-dimensional) systems. The linear propagator of the dynamics (Eq. 2.16) can be approximated in systems with a discrete time by the Jacobi matrix (Eq. (2.23) ). In the following each step of the algorithm is described in detail. 


\section{Linearization:}

The Jacobi matrix $\mathbf{J}_{\mathbf{f}}$ of the underlying equations is needed for the evolution of perturbations in the tangent space (see Eqs. 2.13) - (2.14)). For this purpose, the differential equations which determine the dynamical system need to be linearized, in particular the monodomain equation which includes the local cell dynamics (for example using the Fenton-Karma model Eqs. 2.53 - 2.55) 19 Since the Jacobi matrix acts on each node of the system (with $m$ nodes), it has the dimension $m \times m$ in the end.

\section{Initialization:}

Orthonormal perturbation vectors have to be initialized randomly, which will then be evolved in time (in addition to the actual trajectory). The number $k$ of vectors should be equal to the number of desired Lyapunov exponent: ${ }^{20}$. Since the perturbation vectors have the dimension $m$ of the state vector $\mathbf{x}$, a perturbation matrix $\mathbf{Q}_{\text {Init }}$ can be created by the collection of the perturbation vectors. Hence the matrix including the perturbations has the dimension $m \times k$.

\section{Evolution:}

In parallel to the usual evolution of the state vector $\mathbf{x}$ (actual simulation trajectory), the perturbation matrix $\mathbf{Q}_{\text {Init }}$ is evolved by multiplication with the Jacobi matrix ${ }^{21} \mathbf{J}_{\mathbf{f}}^{i}$, concerning the underlying differential equations $\mathbf{f}$ :

$$
\mathbf{Q}_{i+1}^{*}=\mathbf{J}_{\mathbf{f}}^{i} \mathbf{Q}_{i}
$$

where the index $i$ denotes the simulation step (thus numerical time step). In comparison to $\mathbf{Q}$, the notation of $\mathbf{Q}^{*}$ takes care for the fact, that in practice due to numerical inaccuracies the perturbation vectors of the evolved state $\mathbf{Q}_{i+1}^{*}$ are not orthogonal anymore. The next step of this algorithm takes care for this issue. However, with Eq. (2.74) the system of perturbation vectors is carried along the actual simulation.

\section{Decomposition:}

After a specific number of simulation steps $n_{\text {steps }}$ (the actual choice of $n_{\text {steps }}$ will be discussed later), the perturbation matrix is decomposed by a $Q R$ decomposition into an orthogonal matrix $\mathbf{Q}$ and an upper triangular matrix $\mathbf{R}: \mathbf{Q}_{i}^{*}=\mathbf{Q}_{i} \mathbf{R}_{i}$. This decomposition is essential for the whole algorithm in many ways.

- The logarithms of the diagonal elements of $\mathbf{R}_{i}$ provide the local (thus during the time defined by the $n_{\text {steps }}$ simulation steps) growth rates corresponding to the perturbation

\footnotetext{
${ }^{19}$ The linearization of the local cell model is straightforward by taking partial derivatives. Actually, the discrete version of the Laplace operator (diffusion) is linear already (see section 2.4.3 on page 52, thus it can be used in its original form.

${ }^{20}$ With this method, the largest $k$ Lyapunov exponents are determined, since a perturbation will always grow with the largest Lyapunov exponent corresponding to the associated subspace of the perturbation (see section 2.2 .4 on page 34 .

${ }^{21}$ Since the Jacobi matrix depends on the system state $\mathbf{x}$ it needs to be determined explicitly before each simulation step.
} 
vectors. They need to be extracted and stored. Based on these local growth rates, the final finite time Lyapunov exponents can be extracted.

- Since in numerical simulations the perturbation vectors are not orthogonal from the beginning in an analytical sense, their (numerical) orthogonal alignment deteriorates. Numerically, the vectors are not perfectly orthogonal specifically concerning the first vector, which grows with the largest Lyapunov exponent. That is, why perturbations corresponding to Lyapunov vectors of higher orders also start to grow (and rotate) concerning the largest Lyapunov exponent. Here, the QR decomposition restores the orthogonality of the perturbation vectors and suppresses the alignment of all vectors along the Lyapunov vector which corresponds to the largest exponent.

The perturbation vectors $\mathbf{Q}_{i}^{*}$ which are now orthogonal again (in a numerical sense), need to be renormalized and are then used for the following calculations.

\section{Repetition:}

Steps 3. and 4. are repeated as long as the Lyapunov spectrum needs to be calculated.

\section{Averaging:}

After the simulation, the extracted logarithms of the diagonal elements of $\mathbf{R}_{i}$ are weighted to the length of the corresponding time interval $\left(n_{\text {steps }} \cdot d t\right)$, and averaged over the total length of the calculation time. By this procedure, the finite time Lyapunov exponents are determined:

$$
\lambda_{i}^{\mathrm{Av}}=\frac{1}{N_{\text {Ortho }}} \sum_{k=1}^{N_{\text {Ortho }}} \frac{\lambda_{i}^{(k)}}{n_{\text {steps }} d t} .
$$

The above procedure presents the steps of the algorithm, while in the following we give further details about the calculation:

- The $\mathrm{QR}$ decompositions do not need to be performed after each simulation step. In order to increase the speed of simulations, one can choose a certain number of steps $n_{\text {steps }}$ between two decompositions. However, the longer this interval is, the less the orthogonality of the perturbation vectors is guaranteed, due to numerical inaccuracy. That means, the number of steps $n_{\text {steps }}$ needs to be adjusted to this effect. The maximum of the non-diagonal entries of $\mathbf{R}_{i}$ provides a quantity, which can here be used to estimate how non-orthogonal the perturbations are.

- The perturbation vectors are initialized randomly. In order to provide a finite amount of time for the alignment of the initial perturbation vectors (they converge to the Lyapunov vectors for $t \rightarrow \infty$, see section 2.2 .4 on page 34 for details), it is convenient to discard a fixed amount of time at the beginning of the calculation, where the logarithms of the diagonal elements of $\mathbf{R}_{i}$ are not taken into account concerning the final average. 
- In theory, Lyapunov exponents are defined by an infinite time limit. In numerical simulations, only finite amounts of calculations are feasible. A suitable length of the simulation depends on the respective dynamics of the dynamical system (in the case of a chaotic attractor, the trajectory needs to sample the whole attractor) or the choice of model parameters. In practice, the calculation can be finished, when the average Lyapunov exponents do not change significantly anymore 22

\section{Calculation of the Electrocardiogram}

The electrocardiogram (ECG) can be measured experimentally (shown e.g. in Fig. 1.5 on page 6) and also computed numerically on the basis of excitation pattern distributions of the heart. That is, why the ECG represents a highly important quantity, connecting the experiment to numerical simulations. There are different model approaches for the calculation of an ECG from numerical data. In this thesis, we use the integrated mean of the membrane potential in two-dimensional simulations

$$
\mathrm{ECG}_{\text {Mean }}=\frac{1}{A} \int_{A} V_{\mathrm{m}}\left(\vec{r}^{\prime}\right) d^{2} r^{\prime}
$$

and a more sophisticated version, which is based on the volume conductor approach [87]:

$$
\operatorname{ECG}_{\text {Sim }}(\vec{r})=\int_{V} D \frac{\nabla V_{\mathrm{m}}\left(\vec{r}^{\prime}\right) \cdot\left(\vec{r}^{\prime}-\vec{r}\right)}{\left|\vec{r}^{\prime}-\vec{r}\right|^{3}} d^{3} r^{\prime}
$$

where $D$ is the diffusion constant. Equation 2.77 is used in the study of section 3.3 on page 102, where a more realistic calculation of the electrocardiogram is essential for the respective scientific objective of the study. Figure 2.27 shows an exemplary excerpt of an electrocardiogram time series of an episode of simulated spatio-temporal chaos from that study.

\subsubsection{Software Package MediaSim}

The majority of numerical simulations which are part of this thesis were conducted using the software package "MediaSim" (written in the C++ programming language), was developed in the "Research Group Biomedical Physics". It is a module based tool, with the general purpose of (numerically) solving different kinds of partial differential equations on spatially extended domains (one- two- and three-dimensional). The module based setup is one of the main strengths of the software: In practice, various modules can be chosen from a large amount of available tools. These modules are grouped in e.g. "states" (provides the full information about the state of the system at a specific time), "updaters" (modifies the state due to the choice of the underlying differential equations) or "analyzers" (extracts the

\footnotetext{
${ }^{22}$ In practice, the calculation is finished, when the computed estimates of Lyapunov exponents do not change significantly anymore, i.e. when the obtained values do not exhibit any drift (anymore) and their fluctuations are below some given threshold.
} 


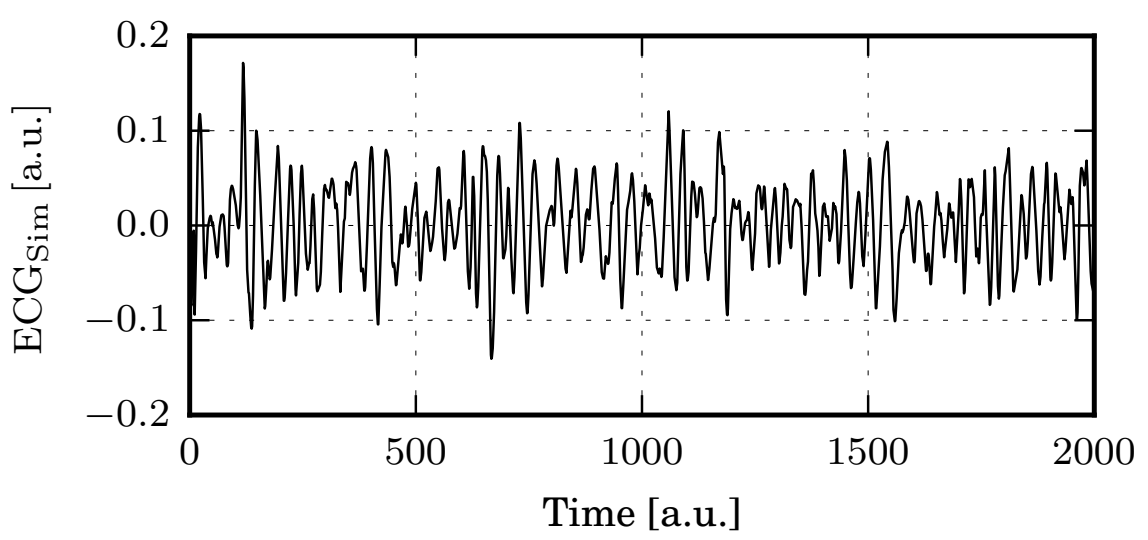

Figure 2.27: Example of the pseudo $\mathrm{ECG}_{\mathrm{Sim}}$ (Eq. (2.77) ) based on numerical data obtained from simulations using the Fenton-Karma model. For more details of the underlying simulation, see section 3.3 on page 102 and the corresponding study.

relevant quantities and information of the simulation, which are crucial for the scientific objective of the study). Due to the structure of the differential equations, additive terms can be included or removed from the dynamics, just by including or removing an updater (e.g. the diffusion, or the local cell dynamics in Eq. 2.45)). For example, the exchange of the local cell model in an already existing simulation framework reduces to the exchange of the "updater" which is responsible for the local cell dynamics, without changing the rest of the simulation code. In this way, I benefited from the already existent software framework, and could extend and enhance the software package.

\subsubsection{The Hardware}

All simulations presented in this thesis were conducted on a computer cluster. It contains of four front end nodes (each 500 GB RAM and 32 cores) and 96 back end nodes (each 64 GB RAM and 16 cores). With this hardware setup it was possible to perform a large amount of simulations with a moderate level of complexity and size at the same time (total number of cores of the back end: 1536), but also a reasonable number of more sophisticated simulations at the same time using parallel computing. 


\section{Chapter 3}

\section{Results}

In this section, the results of four studies are presented. Each study is introduced by stating the respective scientific objective and a short summary about the simulation features and models used. Furthermore, the research question is additionally depicted in a schematic drawing of the state space, which is originally based on Fig. 2.12, where the sinus rhythm was set into the context of the state space. After formulating the scientific objective in each study (e.g. in Fig. 3.1 in the first section of this chapter), the respective result of each study is summed up at the end of each study as a conclusion and also sketched in the state space (e.g. in Fig. 3.2.

Subsequently, the objective of the next results section is formulated in the "updated" state space. Although the sketches are far away from depicting an accurate state space, this procedure should demonstrate the relation of the studies among each other, aims at creating an overall picture of the dynamics and in this way improves the general understanding of the complex dynamics studied in this thesis.

The first three of the four studies are based on manuscripts which are currently at different stages of the publication process in peer reviewed journals (published, submitted and under revision). For this reason, each study is accompanied by a paragraph which states the current status of the manuscript (at the submission date of this thesis) and the individual contributions of the authors. 


\subsection{Features of Chaotic Transients in Excitable Media}

\section{Status of the manuscript}

This manuscript was published in the journal Physical Review Letters on the 4th of August 2017 (Volume 119, Issue 5) 88.

\section{Author Contributions}

- Thomas Lilienkamp designed the research, performed all numerical simulations, analyzed the data and wrote the manuscript.

- Jan Christoph provided a numerical algorithm to identify phase singularities and filaments from excitation data.

- Ulrich Parlitz designed the research and wrote the manuscript.

\section{Scientific Objective}

The aim of this work is to investigate basic properties of spatio-temporal chaos in excitable media. Inspired by the observation that in ev-vivo heart experiments [55] induced ventricular fibrillation terminated by itself, without any interaction (e.g. a defibrillation attempt) from outside, we want to investigate whether this behavior is reproducible in numerical simulations. From the perspective of nonlinear dynamics the equivalent question is whether chaos is persistent or transient in these systems (the phenomenon of transient chaos was discussed in detail in section 2.2.3 on page 30). Although one could imagine in the first place that this difference is rather subtle and more of theoretical nature, it is a first step of understanding the governing mechanism which is responsible for the perpetuation of the dynamics. These investigations are inevitable in order to understand and control the complex dynamics we observe in simulations and experiments.

Concerning the state space of cardiac dynamics, we already interpreted the sinus rhythm using terms from nonlinear dynamics (e.g. the fixed point (resting state) and its basin of attraction) in Fig. 2.12. Using this conception as a starting point, we now want to investigate typical trajectories which describe spatio-temporal chaos and thus enter a different region of the state space (Fig. 3.1).

\section{Numerical Models}

This study is a fundamental investigation of the dynamics of spiral and scroll waves as the main building blocks of the spatio-temporal dynamics and in particular their interaction among each other. For this reason, numerical simulations are performed on generic rectangular (cubic) domains in two (three) dimensions. Interactions with non-trivial boundaries (e.g. the realistic geometry of a heart) are neglected in order to separate different effects and to focus on the pure dynamics and interaction of spiral and scroll waves. However, in order to exclude that results are only valid for a specific cell model, two models are investigated, 


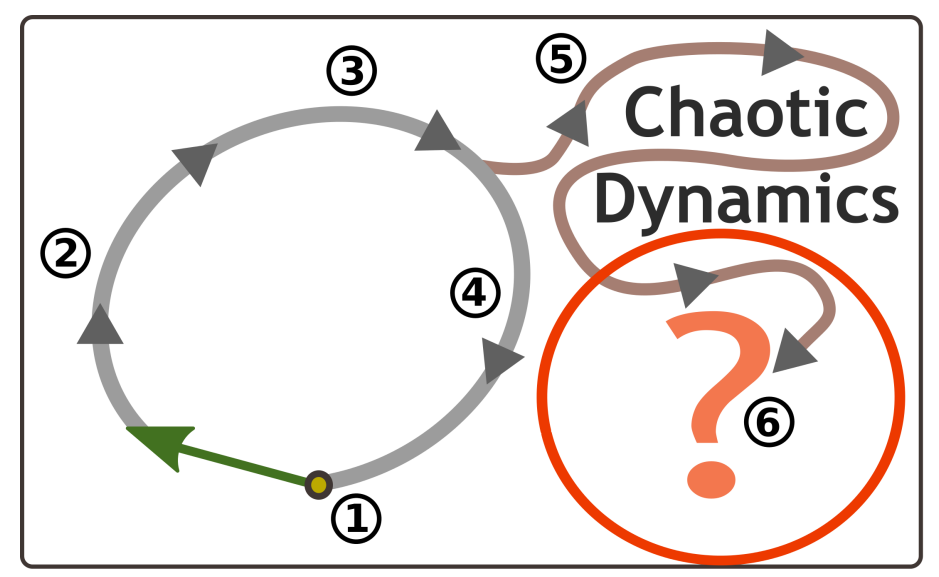

Figure 3.1: The (schematic) state space, formulating the scientific objective concerning the study "Features of Chaotic Transients in Excitable Media Governed by Spiral and Scroll Waves". The sinus rhythm is already included: The start of a contraction cycle is initialized by the stimulus propagating from the av node (section 2.1.2 on page 17). This "perturbs" the system out of the resting state (1) and the electrical excitation wave propagates through the tissue (see Fig. 2.12 for details). When the excitation wave travels through the heart $((2)$ and (3)) it can either return to the resting state (4) or, enter another region of the state space (5), which exhibits chaotic dynamics. We associate this region of the state space with ventricular fibrillation. In the following study, we want to investigate this dynamics, in order to understand what the governing mechanisms for the perpetuation of the dynamics in this part of the state space (6).

the Aliev-Panfilov model and the Fenton-Karma model. The use of two models and three parameter sets in total (AP1 (with the spacing constant $h=0.8$, time stepping $d t=0.2$ and diffusion constant $\mathrm{D}=0.2), \mathbf{F K} 1$ with $(h=1.0, d t=0.2, \mathrm{D}=0.2)$ and FK2 $(h=1.5$, $d t=0.1, \mathrm{D}=0.2)$ ) strengthens the robustness of the obtained results. Both models can be considered as models with a moderate level of complexity (in particular they are not ionic models, see section 2.4.2 on page 48 which is however sufficient for different forms of wave breakup, resulting in creation and annihilation of spiral and scroll waves, one of the main mechanisms of spatio-temporal chaos in excitable media.

The Supplemental Material to this study can be found in section B.1 on page 135. 


\title{
Features of Chaotic Transients in Excitable Media Governed by Spiral and Scroll Waves
}

\author{
Thomas Lilienkamp, ${ }^{1,2,}$ Jan Christoph, ${ }^{1}$ and Ulrich Parlitz ${ }^{1,2}$ \\ ${ }^{1}$ Max Planck Institute for Dynamics and Self-Organization, Am Fassberg 17, 37077 Göttingen, Germany \\ ${ }^{2}$ Institute for Nonlinear Dynamics, Georg-August-Universität Göttingen, Friedrich-Hund-Platz 1, 37077 Göttingen, Germany
}

(Received 19 February 2017; revised manuscript received 16 May 2017; published 31 July 2017)

In excitable media, chaotic dynamics governed by spiral or scroll waves is often not persistent but transient. Using extensive simulations employing different mathematical models we identify a specific type-II supertransient by an exponential increase of transient lifetimes with the system size in 2D and an investigation of the dynamics (number and lifetime of spiral waves, Kaplan-Yorke dimension). In 3D, simulations exhibit an increase of transient lifetimes and filament lengths only above a critical thickness. Finally, potential implications for understanding cardiac arrhythmias are discussed.

DOI: 10.1103/PhysRevLett.119.054101

Chaotic behavior of dynamical systems is a widespread and well-studied phenomenon. It can be observed in a large diversity of systems from simple low-dimensional regimes up to complex and high-dimensional dynamics. However, in many cases it is of great interest whether the observed chaotic dynamics (both in theoretical models and experiments) are persistent or temporary. From a nonlinear dynamics point of view, the dynamics of the latter one is usually determined by chaotic saddles or repellers, whereas persistent chaos is governed by a chaotic attractor. In practice, chaotic transients occur in various fields like ecology [1], particle advection [2], or chemical reactions [3]. In fact, the difference between transient and persistent chaos can be vital in medicine: In cardiology, cardiac arrhythmias (like ventricular fibrillation) can be associated with highly chaotic spatiotemporal wave dynamics inside the heart [4-7], which is lethal in many cases due to the dysfunctional pumping function. Distinguishing between persistent and transient arrhythmias is thus essential, and it may, in the future, have an impact on the medical treatment and risk assessment of cardiac arrhythmias.

In this work we investigate chaotic transients in extended reaction-diffusion systems of excitable media using two different numerical models that describe the action potential propagation in cardiac tissue: The Aliev-Panfilov model [8] is a two-variable model (five parameters) for cardiac excitation, described by the equations

$$
\begin{aligned}
\frac{\partial u}{\partial t} & =\nabla \cdot \underline{D} \nabla u-k u(1-u)(a-u)-u v, \\
\frac{\partial v}{\partial t} & =\epsilon(u, v)[-v-k u(u-a-1)], \\
\epsilon(u, v) & =\epsilon_{0}+\frac{\mu_{1} v}{u+\mu_{2}} .
\end{aligned}
$$

The Fenton-Karma model [9] [Eqs. (4)-(5)] is a three variable model with fourteen parameters, which comprises an approach for modeling the ion channel dynamics of a cell:

$$
\begin{aligned}
& \frac{\partial u}{\partial t}=\nabla \underline{D} \nabla u-I_{\text {ion }}(u, \mathbf{h}) / C_{m}, \\
& \frac{\partial \mathbf{h}}{\partial t}=\mathbf{g}(u, \mathbf{h}) .
\end{aligned}
$$

The detailed equations that describe the ionic currents $I_{\text {ion }}$ in Eq. (4), and the evolution equations for the gating variables $\mathbf{h}=(v, w)$ [Eq. (5)], can be found in the Supplemental Material [10].

In both models, the first term in Eqs. (1) and (4), respectively, describes the diffusive part of the dynamic system. In our simulations, a scalar and homogeneous diffusion tensor was chosen $(\underline{D}=D=0.2)$. The differential equations, Eqs. (1)-(3) and Eqs. (4)-(5), were solved on a spatial grid (with a model specific spacing constant $h$ ) using an explicit Euler scheme with no-flux boundary conditions.

Different choices of parameters cause diverse behavior of spiral or scroll waves (concerning, for example, breakup mechanisms or spiral tip trajectories) [11]. In order to investigate whether properties related to the transient nature of the chaotic dynamics are robust under a change of the local cell dynamic model, and also under a change of parameters, we investigate three distinct cell dynamics: the Aliev-Panfilov model [Eqs. (1)-(3)] from now on abbreviated with AP (solved using a spacing constant $h=0.8$ and $d t=0.2$ ), and the Fenton-Karma model [Eqs. (4)-(5)], using two different parameter sets (all simulation parameters can be found in the Supplemental Material [10]), from now on abbreviated with FK1 (with $h=1.0$ and $d t=0.2$ ) and FK2 (with $h=1.5$ and $d t=0.1$ ), respectively. Both sets of parameters (FK1 and FK2) were investigated in [11] and create a spiral wave breakup by different mechanisms [biphasic action potential duration (APD) restitution curve, and supernormal conduction 


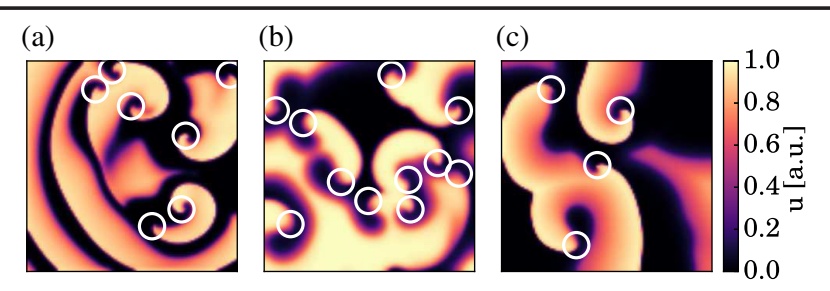

FIG. 1. Snapshots of the spatiotemporal dynamics for the three investigated systems AP (a), FK1 (b), and FK2 (c) (domain sizes $L_{x} \times L_{y}$ of $80 \times 80,100 \times 100$ and $150 \times 150$, respectively). (White) circles indicate the phase singularities (organizing centers) of the spiral waves.

velocity, respectively]. Figure 1 shows snapshots of the chaotic dynamics (variable $u$ ) of the discussed models (AP, FK1, and FK2) in a rectangular two-dimensional domain.

The escape rate $\kappa$ is the quantity that measures how fast random initial conditions (which are governed by the chaotic dynamics) escape the chaotic saddle and reach the final (nonchaotic) state. By generating many initial conditions and determining the fraction that still shows chaotic dynamics at time $t, N_{\mathrm{Ch}}(t)$, the escape rate $\kappa$ can be extracted, since this quantity typically decreases exponentially in time with $N_{\mathrm{Ch}}(t) \sim \exp (-\kappa t)$ [12].

As a first step, we investigated the role of the system size in 2D simulations. In [13] two types of supertransients (systems where the escape rate decreases rapidly with the system size) are distinguished. In systems of type-I supertransients (nonstationary transients), the number of objects which are essential for the chaotic dynamics (e.g., "defects" or "regions of turbulence") decreases in time, and the dynamics converge over time to the final state. For this class of systems, the dependence of the escape rate $\kappa$ on the system size $L$ can usually be described by a power law [Eq. (6), with $\beta>0$ ]. In systems that show transients of type-II in comparison, the transition to the final attractor is abrupt, and cannot usually be predicted by quantities like time series. The escape rate $\kappa$ increases exponentially with the system size $L$ [Eq. (7), with the parameters $a>0$ and $\gamma>0]$

$$
\begin{aligned}
& \kappa(L) \sim L^{-\beta}, \\
& \kappa(L) \sim \exp \left(-a L^{\gamma}\right) .
\end{aligned}
$$

Instead of the escape rate $\kappa$ in the following the inverse escape rate is considered, which is an estimate for the average transient lifetime $\langle T\rangle \approx 1 / \kappa$ [12].

In $2 \mathrm{D}$ simulations on a rectangular domain, the average transient lifetime was determined for various sizes of the 2D simulation area. While keeping the grid spacing $h$ constant for each model, the simulation domain $L_{x} \times L_{y}=\left(N_{x} h\right) \times\left(N_{y} h\right)$ was increased by changing the number of grid points $\left(N_{x} \times N_{y} \in\right.$ $[80 \times 80,90 \times 90,100 \times 100,110 \times 110,120 \times 120,130 \times 130])$. For each domain size, 3000 initial conditions were
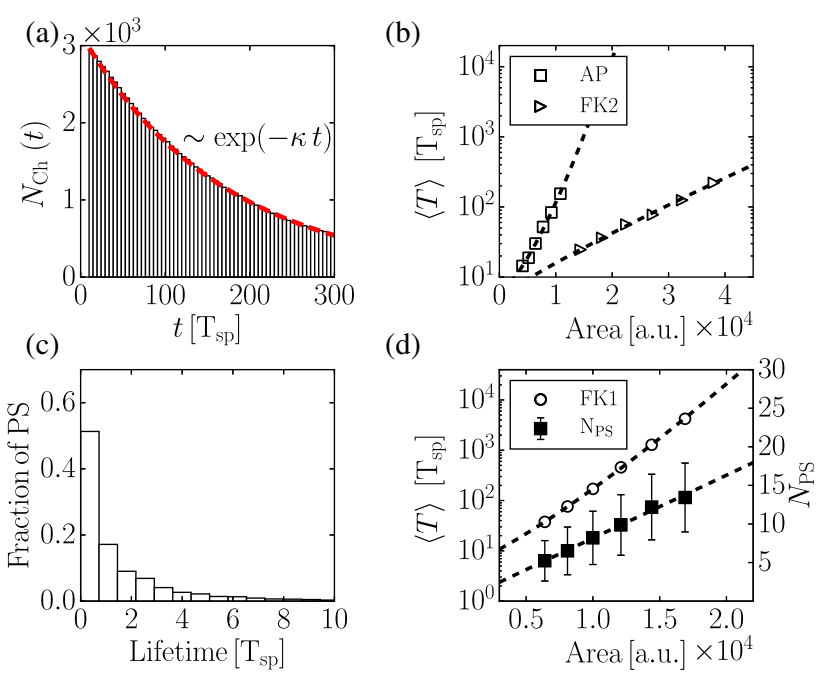

FIG. 2. Chaotic transients and the average lifetime in 2D simulations. In subplot (a) $N_{\mathrm{Ch}}(t)$ is shown over time [measured in spiral rotations $\left(T_{\mathrm{sp}}\right)$ ] for an exemplary domain size of $L_{x} \times L_{y}=100 \times 100$ using the FK1 model. The average transient lifetime $\langle T\rangle \approx 1 / \kappa$ can be extracted by fitting the exponential decay [(red) dashed line]. In (b) the average transient lifetimes are plotted for various system sizes for both models: AP (open square) and FK2 (right pointed triangle). For FK1, the lifetime distribution of phase singularities for a simulation domain of $L_{x} \times L_{y}=100 \times 100$ was determined (c) as well as the mean number of phase singularities $N_{\mathrm{PS}}$ (filled square) and the average transient lifetime $\langle T\rangle$ (open circle) for various area sizes (d).

created (details about the induction protocol can be found in the Supplemental Material [10]). Self-termination of a simulation was declared when the overall excitation (dynamic variable $u$ ) came below a threshold $\left(\left(1 / L_{x} \times L_{y}\right) \sum_{i, j} u_{i j}<0.001\right)$. For the determination of $\langle T\rangle$ from $N_{\mathrm{Ch}}(t)$ an initial amount of time, which is equal to 10 spiral periods, was discarded. The average transient lifetime was determined for all three models (AP, FK1, FK2) for the different domain sizes.

Figure 2(a) shows $N_{\mathrm{Ch}}(t)$ exemplary for FK1 and a domain size of $L_{x} \times L_{y}=100 \times 100$. An exponential scaling of the average transient lifetime with the domain size (area $\left.=L_{x} \times L_{y}\right)$ in 2D was confirmed in all three models [Fig. 2(b) for FK2 and AP, and Fig. 2(d) for FK1, respectively]. In fact, supertransients of type-II were identified with coefficients $\gamma_{\mathrm{AP}}=1.3843 \pm 4.2 \times 10^{-3}$, $\gamma_{\mathrm{FK} 1}=1.2274 \pm 6.9 \times 10^{-4}, \quad \gamma_{\mathrm{FK} 2}=0.8813 \pm 2.8 \times 10^{-2}$, $a_{\mathrm{AP}}=4.5851 \times 10^{-6} \pm 8.6 \times 10^{-11}, a_{\mathrm{FK} 1}=4.4123 \times 10^{-5} \pm$ $1.3 \times 10^{-10}$ and $a_{\mathrm{FK} 2}=7.1151 \times 10^{-4} \pm 1.5 \times 10^{-6}$. It is noteworthy, that the actual scaling parameter $\gamma$ is not only determined by the choice of the cell model (Aliev-Panfilov, Fenton-Karma) but also sensitively depends on the choice of model parameters.

The identification of supertransients of type-II can also be confirmed in the underlying dynamics of the investigated excitable systems: The chaotic dynamics are 
mainly determined by spiral waves in two-dimensional (2D) systems or scroll waves in three-dimensional (3D) systems. The number of the organizing centers of these waves fluctuates during a chaotic episode due to pairwise creation or annihilation of phase singularities (tips of the spirals), wave breakup, or collisions with the boundary. The dynamics terminate (without any impact from outside), if at some point in time every spiral wave annihilates with another spiral wave or the boundary. Only plane waves remain without any phase singularity and the excitation dies out; thus, the chaotic episode has finished and the system remains in the (stable) attractor given by a nonexcited medium (see the Supplemental Material for the course of such a self termination [14]). During such an episode, the number of spiral waves, or their corresponding phase singularities (which play the role of the "defect" here), does not decrease over time, but fluctuates due to the creation and annihilation mechanisms. The lifetime distribution of a single phase singularity for FK1 and a simulation domain of $L_{x} \times L_{y}=100 \times 100$ is shown in Fig. 2(c), indicating that the dynamics are not dominated by single long-living spiral waves but characterized by a constant production and annihilation of relatively short-living phase singularities. As already noted by Strain and Greenside [15], the final collapse of the system occurs then, abruptly, and no obvious indications for the upcoming termination can be found. For exemplary time series of the number of phase singularities $N_{\mathrm{PS}}$ and the pseudo ECG before the collapse of the dynamics, as well as details of the detection of the phase singularities, see [10].

When the simulation area is extended, the mean number of phase singularities (and thus the number of spiral waves) increases linearly [Fig. 2(d)], which is in accordance with the findings that spiral waves occupy finite amounts of the area, also called "tiles" [5,16]. The lifetime distribution of single spirals does not change with the system size (mean lifetime of spiral waves $\left\langle T_{\mathrm{PS}}\right\rangle=$ $4.49,4.37,4.48,4.45,4.54,4.53 \mathrm{~T}_{\mathrm{sp}}$ for $L_{x}=80$, 90, $100,110,120,130)$. Using a Markovian approach for the dynamics (each state characterized by the number of phase singularities), and assuming that the transition probability to a state with no spiral waves is decreasing exponentially with the number of spiral waves (which grows linearly with the system size), one can reasonably deduce the exponential scaling of the transient lifetime with the system size here [17]. From this point of view, the scaling parameters $a$ and $\gamma$ can be related to the lifetime of the spiral waves and the number or the size of single spiral waves compared to the domain size.

Apart from the average transient lifetime, which is a characteristic feature of the transient nature, we focus in the following on the chaotic properties of the dynamics. In systems which exhibit chaotic transients, an initial condition after a finite amount of time will end up in another attractor (which actually can also be chaotic). However,
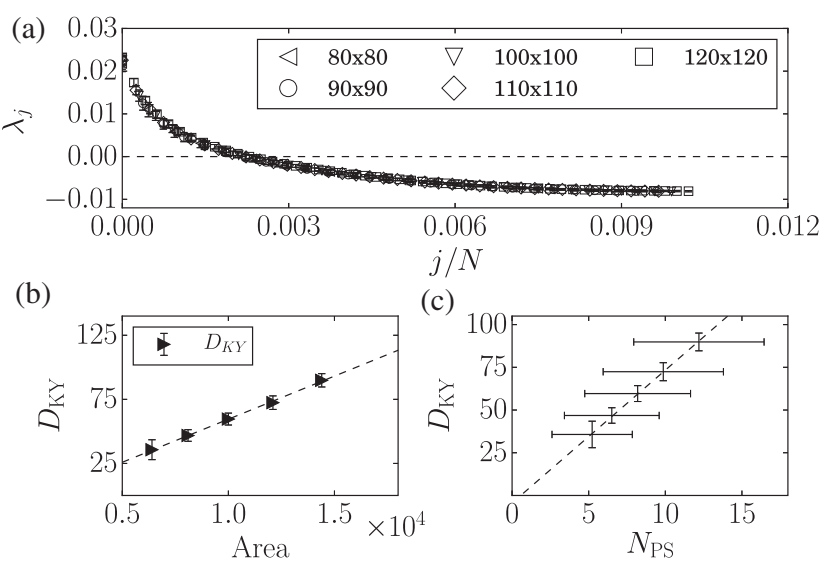

(c)

FIG. 3. The chaotic features of the dynamics using FK1. Analysis of the Lyapunov density for various system sizes (a), Kaplan-Yorke dimension $D_{\mathrm{KY}}$ (filled triangles) versus the system size (b) and $D_{\mathrm{KY}}$ versus $N_{\mathrm{PS}}$ (c).

dynamic invariants that are used for "classical" (persistent) chaotic systems can, in some cases, also be used for characterizing the transients. For example, Lyapunov exponents can, in practice, also be calculated in systems with finite chaotic episodes. Because Lyapunov exponents are mathematically defined on an infinite time scale, we refer to "finite time Lyapunov exponents" when we speak of Lyapunov exponents of chaotic transients [12]. Technically, there is no difference in the calculation scheme, except that the calculation stops before the self termination of the dynamics. However, in practice this is only meaningful if the transients provide enough time for the convergence of the estimates of the exponents.

The Lyapunov spectrum provides information about how chaotic the dynamics are before self termination. We investigated how these properties of the dynamics change with an increasing system size (details can be found in the Supplemental Material [10]). Figure 3(a) depicts the Lyapunov density $([12,18])$ (jth Lyapunov exponent versus $j$ divided by system size $N=N_{x} \times N_{y}$ ). The Lyapunov exponents for different system sizes clearly align with each other, which implies that the number of positive or negative Lyapunov exponents scales with the system size (extensive chaos).

Furthermore, in order to assess the chaotic dynamics, the Kaplan-Yorke dimension $D_{\mathrm{KY}}$ was calculated for various system sizes based on the Lyapunov spectrum [19]. Figure 3(b) shows that the Kaplan-Yorke dimension $D_{\mathrm{KY}}$ grows linearly with the system size (triangles). Since the number of phase singularities (equivalent to the number of spiral waves) also grows linearly [Fig. 2(d)], we can identify a mean $D_{\mathrm{KY}}$ per spiral wave of $\approx 7.77 \pm 0.12$. Although studies indicate that parts of the degrees of dynamic freedom in spatiotemporal chaos in excitable media are not related to the defects (spiral cores) [20], we can conclude that the degree of chaos 
(Kaplan-Yorke dimension) can be estimated by the number of spiral waves permitted by the respective system size [see Fig. 3(c)].

Similarly to [21], we also found that noise has an impact on the average lifetime. Details regarding the results can be found in the Supplemental Material [10].

As a next step, the average transient lifetime was also determined in 3D domains. In three dimensions, spiral waves correspond to scroll waves, whereas the former pointlike phase singularities that mark the tip of a spiral wave correspond to filamentlike one-dimensional curves in 3D. Scroll waves can be considered as "stacked spiral waves," where the curvature and the meandering behavior of the filament are essential for the dynamics. In particular, we are interested in how the average transient lifetime changes when extending the 2D dimensional domain step by step to 3D. For this purpose, the initial 2D simulations with $L_{x} \times L_{y}=(80 h) \times(80 h)$ were gradually extended in the third dimension $L_{z}$ (from now on denoted as "thickness"). As in [22], in all three models, a critical thickness in the third dimension could be established up to which the average transient lifetime remains approximately constant (see Fig. 4). Above this threshold, the average transient lifetime increases exponentially.

In order to find the dynamic origin for this critical thickness, the filaments (the organizing centers) of the scroll waves were also detected. Details about the detection of the filaments can be found in the Supplemental Material [10]. In Figs. 4(a), 4(c), and 4(e) the average transient lifetime $\langle T\rangle$ is compared to the average number of filaments, $N_{\text {fila }}$, for each thickness of the domain. In all three models, the critical thickness in the average transient lifetime is also pronounced in terms of the number of filaments $N_{\text {Fila }}$. In addition, the average length of the filaments per thickness $\left(\mathrm{ALF} / L_{z}\right)$ was also determined. In the case of straight filaments only aligned along the third dimension of the system, this quantity is equal to one. Thus, deviations from a constant value provide information about deviating filament alignment. In "thin" systems, the domain only provides enough space for filaments aligning along the third dimension (thus, the system is quasi-2D). The approximately constant ratio $\mathrm{ALF} / L_{z}$ for lower thicknesses indicates that most filaments are aligned along the third dimension [crosses in Fig. 4(b) (AP), 4(d) (FK1), and 4(f) (FK2)]. In larger domains, for AP and FK1, the critical thickness can also be recognized by ALF/ $L_{z}$ (smeared out for FK2). However, for bigger systems, $\mathrm{ALF} / L_{z}$ saturates in all three models (or even slightly decreases for AP and FK1), indicating that the maximum (average) filament length is (model dependent) confined. The presence of the critical thickness in both quantities $\left(N_{\text {fila }}\right.$ and $\left.\mathrm{ALF} / L_{z}\right)$ suggests that above the critical thickness, filaments break up (due to a negative filament tension in all models [9,23]). This transition from vertically arranged filaments to actual scroll wave turbulence above a critical thickness of the substrate
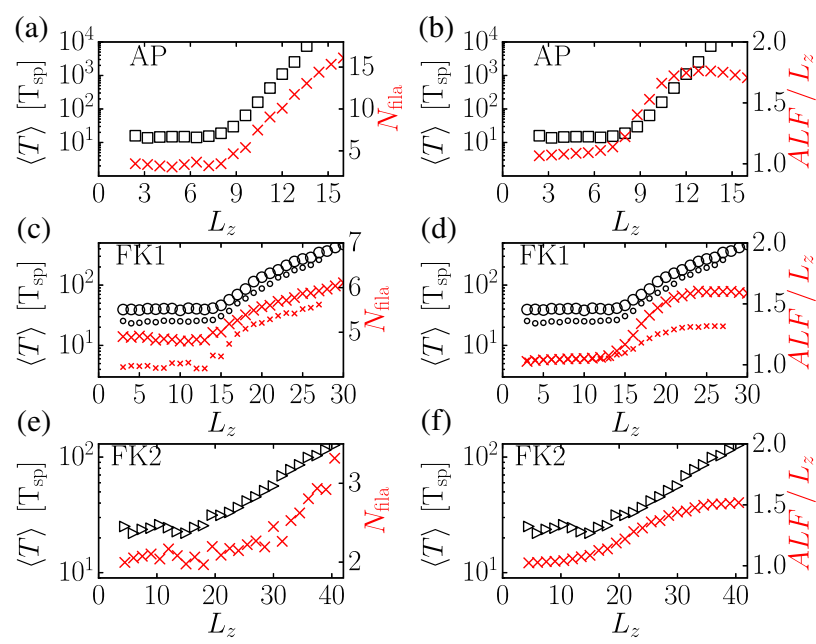

FIG. 4. Chaotic transients in 3D domains. A critical thickness in the third dimension of the simulation grids exists in all three models, below which the average transient lifetime remains approximately constant [AP: (square) in (a) and (b), FK1: (circle) (small circles for simulations using the doubled resolutions) in (c) and (d) and FK2: (right-pointing triangle) in (e) and (f)]. The dynamic origin of the critical thickness concerning the average transient lifetime can be identified with the average number of filaments $\left(N_{\text {fila }}\right)$ in the system, which is rapidly increasing above the critical thickness [(crosses) in (a) (AP), (c) (FK1, small crosses for simulations using doubled resolutions) and (e) (FK2)]. The average length of the filaments divided by the thickness $\left(\mathrm{ALF} / L_{z}\right)$ also exhibits the critical thickness, but saturates for larger domains [(crosses) in (b) (AP), (d) (FK1, small crosses for simulations using the doubled resolutions) and (f) (FK2)].

was also observed by Dierckx et al. [24]. However, the saturation of $\mathrm{ALF} / L_{z}$ leads to the conclusion that the increase of the average transient lifetime is mainly based on the pure number of filaments (equivalent to the number of spiral waves in 2D) and does not depend significantly on the length of the filaments. In order to investigate finite size effects, all simulations were repeated in the case of FK1 with a doubled resolution $[h \rightarrow h / 2$, small circles and crosses in Figs. 4(c) and 4(d)]. Quantitative differences in the absolute numbers (e.g., $N_{\text {fila }}$ ) are visible, but the main qualitative findings (in particular the critical thickness) are robust under a doubling of the spatial resolution.

We have shown that chaotic transients are a robust phenomenon in excitable media and occur similarly in different numerical models (Aliev-Panfilov model and Fenton-Karma model) of excitable systems with different underlying mechanisms for spiral wave breakup. The average lifetimes of chaotic transients depend on the system size, in both two and three dimensions, and are influenced by the addition of low amplitude noise. We found in the investigated excitable systems, that spiral or scroll waves, and their corresponding phase singularities or filaments, are the main topological objects that promote the duration of chaotic episodes. 
Our findings agree with the general notion that larger heart muscle volumes increase the risk of cardiac arrhythmias and related morbidity and mortality [25-27]. Our results suggest that larger volumes can possibly contain a larger number of phase singularities, and therefore, not only increase the transient lifetimes, but also enhances the spatiotemporal complexity of the chaotic dynamics, which may have an impact on the success rates of defibrillation attempts [28,29]. In particular, the role of the critical thickness investigated in the 3D simulations may be of further interest in this context. Cardiac hypertrophy, for instance, is accompanied by an increase in heart muscle volume. Even though the overall number of cardiac cells does not change in hypertrophic hearts, other factors, such as altered excitation thresholds and conduction velocities, may lead to an effective scaling of the size of the excitable system with respect to the sizes of dynamic structures like spiral or scroll waves present in the system. In fact, from this point of view, the administration of certain antiarrhythmic agents could be interpreted as a change of properties of the cardiac tissue in order to promote early self-termination of cardiac arrhythmias [30]. With respect to transient lifetimes, phenomenological modeling could provide a generalized framework for investigating the influence of cardiac substrate changes onto the persistence of cardiac arrhythmias.

We thank the International Max Planck Research School (IMPRS) for Physics of Biological and Complex Systems (PBCS), the Federal Ministry of Education and Research (BMBF, FKZ031A147, GO-Bio), the German Centre for Cardiovascular Research (DZHK e.V.), the "Deutsche Forschungsgemeinschaft (DFG, Collaborative Research Centers SFB1002 "Modulatory Units in Heart Failure", project C03 and SFB937 "Collective Behavior of Soft and Biological Matter", project A18) for financial support. Furthermore, we thank Stefan Luther and Sebastian Berg for fruitful discussions and continuous support.

*thomas.lilienkamp@ds.mpg.de

[1] K. McCann and P. Yodzis, Am. Nat. 144, 873 (1994).

[2] J. J. B. Biemond, A. P. S. de Moura, G. Károlyi, C. Grebogi, and H. Nijmeijer, Phys. Rev. E 78, 016317 (2008).

[3] A. M. Barr, K. Na, L. E. Reichl, and C. Jung, Phys. Rev. E 79, 026215 (2009).

[4] J. M. Davidenko, A. V. Pertsov, R. Salomonsz, W. Baxter, and J. Jalife, Nature (London) 355, 349 (1992).

[5] R. A. Gray, A. M. Pertsov, and J. Jalife, Nature (London) 392, 75 (1998).

[6] F. X. Witkowski, L. J. Leon, P. A. Penkoske, W. R. Giles, M. L. Spano, W. L. Ditto, and A.T. Winfree, Nature (London) 392, 78 (1998).
[7] E. M. Cherry and F. H. Fenton, New J. Phys. 10, 125016 (2008).

[8] R. R. Aliev and A. V. Panfilov, Chaos Solitons Fractals 7, 293 (1996).

[9] F. Fenton and A. Karma, Chaos 8, 20 (1998).

[10] See Supplemental Material at http://link.aps.org/ supplemental/10.1103/PhysRevLett.119.054101 for evolution equations of the Fenton-Karma model, simulation parameters, technical details of the simulations, and further results.

[11] F. H. Fenton, E. M. Cherry, H. M. Hastings, and S. J. Evans, Chaos 12, 852 (2002).

[12] Y.-C. Lai and T. Tél, Transient Chaos, Applied Mathematical Sciences (Springer New York, New York, NY, 2011), Vol. 173.

[13] J. P. Crutchfield and K. Kaneko, Phys. Rev. Lett. 60, 2715 (1988).

[14] See Supplemental Material at http://link.aps.org/ supplemental/10.1103/PhysRevLett.119.054101 for the course of such a self termination on a $100 \times 100$ grid using the FK1 model.

[15] M. C. Strain and H. S. Greenside, Phys. Rev. Lett. 80, 2306 (1998).

[16] G. Byrne, C. D. Marcotte, and R. O. Grigoriev, Chaos 25, 033108 (2015).

[17] K. Sugimura and H. Kori, Phys. Rev. E 92, 062915 (2015).

[18] Dynamical Systems Approach to Turbulence, edited by T. Bohr, Cambridge Nonlinear Science Series Vol. 8 (Cambridge University Press, Cambridge, New York, 1998).

[19] J. L. Kaplan and J. A. Yorke, in Functional Differential Equations and Approximation of Fixed Points, edited by H.-O. Peitgen and H.-O. Walther (Springer, Berlin, Heidelberg, 1979), Vol. 730, pp. 204-227.

[20] D. A. Egolf, Phys. Rev. Lett. 81, 4120 (1998).

[21] R. Wackerbauer and S. Kobayashi, Phys. Rev. E 75, 066209 (2007).

[22] Z. Qu, Am. J. Physiol. 290, H255 (2005).

[23] V. N. Biktashev, A. V. Holden, and H. Zhang, Phil. Trans. R. Soc. A 347, 611 (1994).

[24] H. Dierckx, H. Verschelde, Ö. Selsil, and V. N. Biktashev, Phys. Rev. Lett. 109, 174102 (2012).

[25] J. M. McLenachan, E. Henderson, K. I. Morris, and H. J. Dargie, N. Engl. J. Med. 317, 787 (1987).

[26] T. Kahan, Heart and lung 91, 250 (2005).

[27] A. Verma, A. Meris, H. Skali, J. K. Ghali, J. M. O. Arnold, M. Bourgoun, E. J. Velazquez, J. J. McMurray, L. Kober, M. A. Pfeffer, R. M. Califf, and S. D. Solomon, JACC Cardiovasc. Imaging 1, 582 (2008).

[28] N. Bajaj, L. Leon, S. Kimber, and E. Vigmond, Proceedings of the Annual Conference on Engineering in Medicine and Biology, Shanghai, China, 2005 (IEEE, Shanghai, 2005), pp. 7208-7211.

[29] G. Plank, L. J. Leon, S. Kimber, and E. J. Vigmond, J. Cardiovasc. Electrophysiol. 16, 205 (2005).

[30] M. Yamazaki, H. Honjo, H. Nakagawa, Y. S. Ishiguro, Y. Okuno, M. Amino, I. Sakuma, K. Kamiya, and I. Kodama, Am. J. Physiol. 292, H539 (2006). 


\section{Summary of Results}

In each model, the investigated chaotic dynamics governed by spiral and scroll waves is transient. We can conclude that the underlying object in the state space is most likely not a chaotic attractor, but a chaotic saddle. Thus, we obtained a first insight into the structure of the region of the state space, which exhibits spatio-temporal chaos. Due to the transient nature of the dynamics, a typical (chaotic) trajectory will return to the resting state at some point in time (Fig. 3.2).

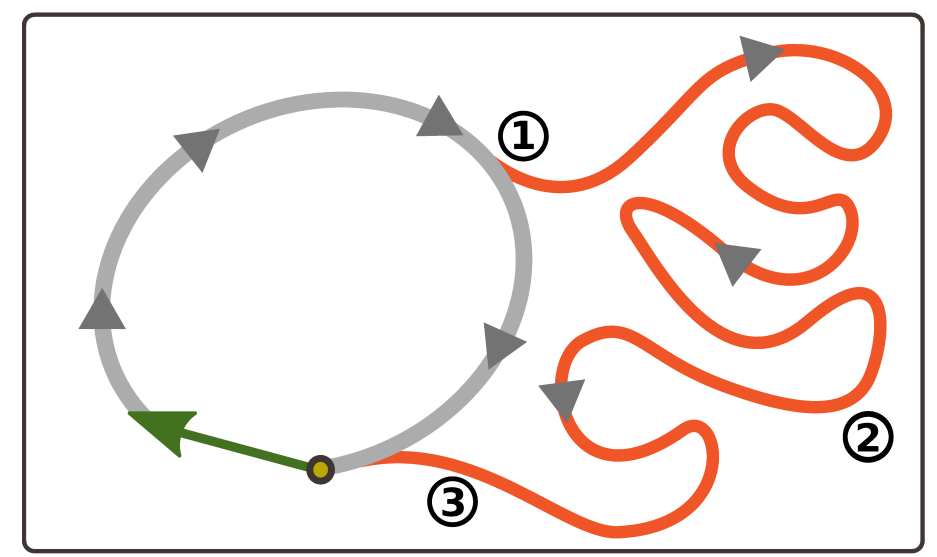

Figure 3.2: The (schematic) state space, depicting the conclusion of the study "Features of Chaotic Transients in Excitable Media Governed by Spiral and Scroll Waves". The finding of this study, that the spatio-temporal chaotic dynamics of the investigated systems is transient, is demonstrated by an exemplary trajectory (red). The chaotic episode starts at some point, where the dynamics deviates from the usual sinus rhythm (1). Subsequent, the trajectory enters another region of the state space, where the dynamics is chaotic (2). Nevertheless, after a certain duration (the lifetime of the episode) the trajectory returns to the resting state (3) at some point.

This includes, that similar to the trajectory of the sinus rhythm (discussed in section 2.2.2, also the chaotic trajectory is located inside the basin of attraction of the resting state. However, the structure in the corresponding region of the state space is significantly different from the region in the proximity of the trajectory representing the sinus rhythm. This results in a different transient lifetime in comparison to the regular dynamics of the sinus rhythm 1 . Due to the chaotic dynamics determined by the chaotic saddle, also the lifetime of two typical chaotic trajectories is different due to the sensitivity of initial conditions. For this reason, the average lifetime $\langle T\rangle$ (averaged over many trajectories) was determined, which characterizes the dynamic. We investigated the scaling behavior of $\langle T\rangle$ concerning the domain size in two and three dimensions, and could characterize the transient chaotic dynamics of spatially extended systems as type-II supertransients [43]. Furthermore, we investigated the underlying mechanism, explaining the transient nature of the dynamics, and related them to quantities from the nonlinear dynamics (Kaplan-Yorke dimension [47]).

\footnotetext{
${ }^{1}$ The time from the induction of the sinus rhythm by the av node back to the resting state (equivalent to a whole contraction cycle of the heart) is approximately constant.
} 


\subsection{Terminal Transient Phase of Chaotic Transients}

\section{Status of the manuscript}

The following section is based on a submission of a manuscript.

\section{Author Contributions}

- Thomas Lilienkamp designed the research, performed all numerical simulations, analyzed the data and wrote the manuscript.

- Ulrich Parlitz designed the research and wrote the manuscript.

\section{Scientific Objective}

In the previous study, the transient nature of spatio-temporal chaos in excitable media was investigated. The average transient lifetime $\langle T\rangle$ is the quantity which is highly relevant for the clinical application: the duration of an episode of ventricular fibrillation can make the difference between life and death.

Another key feature of the transient dynamics is the actual process of self-termination, the transition from chaos to the resting state. So far, the collapse of the dynamics appears to be spontaneous, and is not easily recognizable in quantities like e.g. the number of spiral waves, or the pseudo ECG (see for example Fig. 3 of the Supplemental Material of the previous study in section B.1 on page 135). In this study, we quantify the collapse of the chaotic dynamics and the return to the (non-chaotic) attractor. In particular, we investigate whether a "transition phase" between the chaotic dynamics and the resting state exists (Fig. 3.3). Such a transition zone would provide then the general basis for possible observables which could predict a self-termination of a chaotic episode.

We probe the state space by using small perturbations, applied to trajectories which are close to their self-termination. With the analysis of the lifetimes of those perturbed trajectories, we gain information about its structure.

Apart from cardiac models, we consider also further models of spatially extended systems of the field of neuroscience and chemical reactions in order to validate our results to a broad class of systems. Furthermore, we investigate the final phase before self-termination also in low-dimensional maps, where the governing processes which also might be relevant for the more complex and high-dimensional systems, can be studied in detail.

\section{Numerical Models}

In order to validate that our findings are not only applicable for models of cardiac dynamics but constitute a rather fundamental effect of transient chaos in general, we investigated several models from various scientific fields. The investigated systems are:

- The Fenton-Karma Model: A model for cardiac electrical wave propagation in cardiac dynamics (using the parameter set FK1 (section A.2 on page 132, with $\mathrm{D}=0.2, h=1.0, d t=0.2)$. 


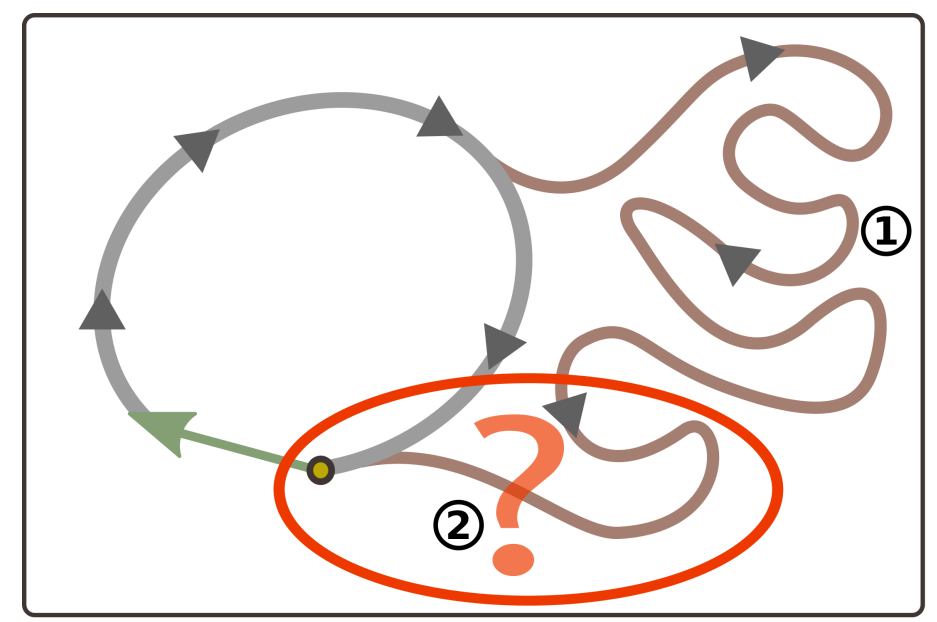

Figure 3.3: The (schematic) state space, formulating the scientific objective concerning the study "Terminal Transient Phase of Chaotic Transients". In the previous study we investigated the transient nature of the spatio-temporal dynamics. In the state space this means, that an exemplary chaotic trajectory (1) will return to the resting state at some point in time. In this study, we are interested in the final phase of these episodes (2). That means, we quantify the state space structure in the proximity of occurring self-termination events of trajectories.

- The Bueno-Orovio-Cherry-Fenton Model: A model for cardiac electrical wave propagation in cardiac dynamics (using the parameter set BOCF1 (section A.5 on page 134, with $\mathrm{D}=0.2, h=1.0, d t=0.1)$.

- Morris-Lecar Neuronal Ring Network: A model for the firing activity of neurons (parameters specified in the study).

- The Gray-Scott Model: A model for a chemical reaction of the species U, V and $\mathrm{P}: \mathrm{U}+2 \mathrm{~V} \rightarrow 3 \mathrm{~V}, \mathrm{~V} \rightarrow \mathrm{P}$ (parameters specified in the study).

- The Tent Map: A simple one-dimensional map, which can exhibit sustained as well as transient chaos.

- The Hénon Map: A two-dimensional invertible map, where the transition from persistent to transient chaos appears via a boundary crises bifurcation.

The number of different models from various fields of nonlinear dynamics should underline the significance of the obtained results, and strengthen the general applicability of the our findings. 


\begin{abstract}
Transient chaos in spatially extended systems can be characterized by the length of the transient phase, which typically grows quickly with the system size (supertransients). For a large class of these systems, so-called type-II-supertransients, the chaotic phase terminates abruptly, without any obvious precursors in commonly used observables. We investigate transient spatio-temporal chaos in four different models of this class. By probing the state space using perturbed trajectories we show the existence of a "Terminal Transient Phase" which occurs prior to the abrupt collapse of chaotic dynamics. During this phase the impact of perturbations is significantly different from the earlier transient and particular patterns of (non-)susceptible regions in state space occur close to the chaotic trajectories. We therefore hypothesize that even without perturbations proper precursors for the collapse of type-IIsupertransients exist which might be highly relevant for coping with spatio-temporal chaos in cardiac arrhythmias or brain functionality, for example.
\end{abstract}

\title{
3.2.1 Introduction
}

Transient chaos can be observed in diverse systems [41, e.g. in ecology [37, turbulence [36], neural networks [38, 39, 40] or statistical physics [89]. Chaotic transients in spatially extended systems are denoted as "supertransients" if the average transient lifetime increases rapidly with the system size [41]. Based on the scaling behavior and the underlying mechanism Crutchfield and Kaneko [43] distinguish between two qualitatively different groups of supertransients: The dynamical evolution of type-I-supertransients is determined by a continuous decrease of "defects" (nonstationary) and an increase of the transient lifetime with the system size by a power-law. In the case of type-II-supertransients, the dynamics can be described as quasi-stationary and the collapse of the chaotic transient (transition to an attractor) is sudden. The average duration of the transients can be characterized here by an exponential scaling with the system size.

In this study we consider four different models which show distinct dynamics described by systems of partial differential equations. Each model exhibits transient spatio-temporal chaos in certain parameter regimes. The Fenton-Karma model [26] and the Bueno-OrovioCherry-Fenton model (BOCF) [90] are two examples of models of excitable media which can be used to describe the action potential propagation in cardiac tissue (Exemplary snapshots of chaotic dynamics are shown in Fig. 3.4(a) and (b), respectively). The Morris-Lecar neuron model is a reduction of the Hodkin-Huxley neuron model [91] and describes the ion currents of the barnacle giant muscle fiber (a transient episode of chaotic dynamics is depicted in Fig. 3.4(c)), whereas the Gray-Scott model (Supplemental Material) can be used to simulate chemical reactions 92 . Type-II supertransients can be characterized by an exponential scaling of the average lifetime depending on the system size. Concerning these features, the investigated systems belong to the group of type-II supertransients in the case of extended excitable media [88, 93, 94] as well as the Morris-Lecar neuron model [95] and the Gray-Scott model [96].

The sudden collapse of the dynamics in these systems is abrupt and can so far not be pre- 


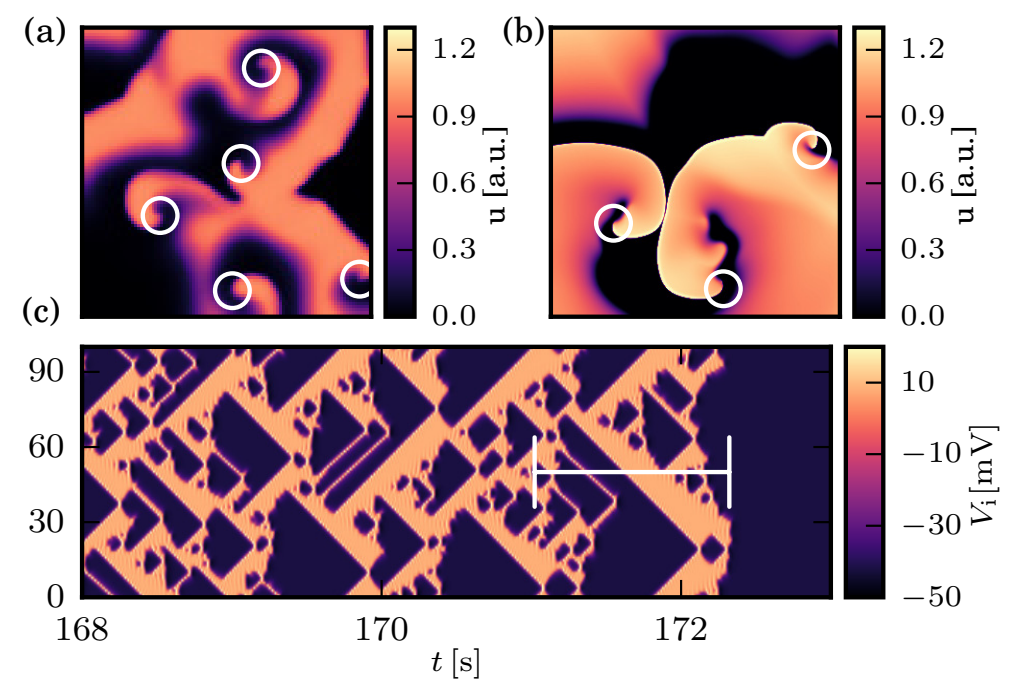

Figure 3.4: Spatio-temporal chaos in the Fenton-Karma model (a), the Bueno-OrovioCherry-Fenton model (BOCF) (b), and the Morris-Lecar neuron model (c), respectively. In the first two cases, a snapshot of the variable $u$ is shown. Here, the dynamics is governed by spiral waves, where white circles indicate phase singularities, which correspond to the tips of the spiral waves. Subplot (c) presents an exemplary episode of (transient) spatiotemporal chaos modeled by the Morris-Lecar neuron model, which ends at $t=172.38 \mathrm{~s}$. The membrane potential $V_{i}$ of $i=1, \ldots$ of $N=100$ neurons is shown here. The white bar depicts the length of the Terminal Transient Phase (TTP), which is discussed in the results section.

dicted [96, 41, 94]. However, by probing the state space using small but finite perturbations of single trajectories we show the existence of a finite time interval before the actual collapse where the coming breakdown is already visible. This period of time is called Terminal Transient Phase (TTP) in the following. The existence of the TTP indicates in contrast to previous conception, that the self-termination of the chaotic dynamics is not fully abrupt and uncorrelated, but the end of a chaotic episode is a process with a finite duration.

\subsubsection{Methods}

For simulations of excitable media, the monodomain approach [97] was used, which describes the evolution of the membrane potential $u$, due to diffusion and the local cell dynamics (first and second term in Eq. (3.1), respectively). In both cardiac models used in this study (Fenton-Karma [26] and Bueno-Orovio-Cherry-Fenton [90]), the cell dynamics is characterized by three currents, which describe the fast inward current $I_{\mathrm{fi}}$, the slow outward current $I_{\mathrm{so}}$ and the slow inward current $I_{\mathrm{si}}$. The actual behavior of the currents and the evolution equations of the slow variables $\mathbf{h}$ (Eq. (3.2)) are model specific. 


$$
\begin{aligned}
& \frac{\partial u}{\partial t}=\nabla \cdot \underline{\mathbf{D}} \nabla u-\left(I_{\mathrm{fi}}+I_{\mathrm{so}}+I_{\mathrm{si}}\right) / C_{m}, \\
& \frac{\partial \mathbf{h}}{\partial t}=\mathbf{g}(u, \mathbf{h}) .
\end{aligned}
$$

\section{Fenton-Karma Model}

The Fenton-Karma model is a three variable model (12 parameters) for generic cardiac action potentials. The expressions for the transmembrane currents $I_{\mathrm{fi}}, I_{\mathrm{so}}$ and $I_{\mathrm{si}}$, as well as the evolution equations of the gating variables $\mathbf{h}=(v, w)$ can be found in section 2.4 .2 on page 48 (Eqs. 2.50) - 2.52), and Eqs. 2.54 - 2.55), respectively).

We assume an isotropic and homogeneous diffusion, thus the diffusive part can be written as $\nabla \cdot \underline{\mathbf{D}} \nabla u=D \Delta u$ with the diffusion constant $D=0.2$. The simulations of the Fenton-Karme model were performed on a rectangular two-dimensional domain with a size of $N_{x} \times N_{y}=$ $100 \times 100$ grid points (spacing constant $h=1.0$ and time step $d t=0.2$ ). No-flux boundary conditions were used. The parameters used for this model can be found in section A on page 131 in Tab. A.2.

\section{Bueno-Orovio-Cherry-Fenton Model}

The second model is the Bueno-Orovio-Cherry-Fenton model [90], which is still not too complex (four variables, 28 parameters), but can be used to simulate action potentials of human ventricular tissue. Equations A.1 - A.3 in section A on page 131 show the expressions for the local currents $I_{\mathrm{fi}}, I_{\mathrm{so}}$ and $I_{\mathrm{si}}$. The evolution equations of the gating variables $\mathbf{h}=(v, w, s)$ can also be found there (Eqs. A.4 - A.13)).

We assume a constant diffusion constant of $D=0.2$. The two-dimensional rectangular simulation domain has a size of $N_{x} \times N_{y}=300 \times 300$ grid points. A spacing constant of $h=1.0$, a time step of $d t=0.1$, and no-flux boundary conditions were used. The parameters used for this model can be found in Tab. A.5.

\section{Morris-Lecar neuronal ring network}

The Morris-Lecar neuronal ring network [91] is a model for the firing activity of neurons and was investigated in terms of the interspike interval variability [98, central pattern generators for asymmetric traveling waves [99]. Furthermore, also the chaotic dynamics was studied [100]. Equation (3.3) describes the evolution of the membrane potential $V_{i}$ of neuron $i$, which changes due to the local ion concentrations (first term) and by diffusive coupling (second term). Furthermore, the evolution of the number of open potassium channels $n_{i}$ is given by Eq. (3.4. 


$$
\begin{aligned}
\frac{\partial V_{i}}{\partial t} & =\frac{I-I_{L}\left(V_{i}\right)-I_{C a}\left(V_{i}\right)-I_{K}\left(V_{i}\right)}{C}+D \Delta V_{i}, \\
\frac{\partial n_{i}}{\partial t} & =\frac{n_{s s}\left(V_{i}\right)-n_{i}}{\tau_{n}\left(V_{i}\right)}
\end{aligned}
$$

The underlying equations describing the ion currents and the gating variables as well as the parameter set used are given in the Supplemental Material in section B.2 on page 139 (Eqs. B.1 - B.6) and Tab. B.1. respectively). Simulations of a one dimensional ring of Morris-Lecar elements were performed using $N=100$ neurons, a diffusion constant of $D=0.05$, a spacing constant of $h=1$ and a time constant of $d t=0.2$, where periodic boundary conditions were used. With the parameters chosen here, the chaotic dynamics can either collapse to the rest state or (due to the periodic boundary conditions) to a state of pulse propagation. Since we concentrate here on the end of the chaotic regime, we consider both cases as the end of the chaotic dynamics.

\section{Tent Map}

The tent map is a simple one-dimensional map, described by

$$
x_{n+1}= \begin{cases}a x_{n} & \text { for } x<\frac{1}{2} \\ a\left(1-x_{n}\right) & \text { for } x \geq \frac{1}{2}\end{cases}
$$

\section{Hénon Map}

The Hénon map is defined by the following equations

$$
\begin{aligned}
& x_{n+1}=a+b y_{n}-x_{n}^{2}, \\
& y_{n+1}=x_{n} .
\end{aligned}
$$

In this study, we use $b=0.3$ and varied the parameter $a$ in order to achieve transient chaos.

\subsubsection{Results}

In the next section the transient features of the spatio-temporal dynamics are shown and the average lifetime $\langle\mathrm{T}\rangle$ of those transients is determined. Subsequently we investigate the structure of the state space, by perturbing single trajectories of spatio-temporal chaos and analyze how the lifetime of those perturbed trajectories changes. Eventually, we investigate how the lifetime of perturbed trajectories changes, when the perturbation is located close (in time) to the self-termination of the original trajectory. The change of the lifetime distributions of the perturbed trajectories close to the collapse is discussed, as well as the spatial structures of the perturbation space before self-termination. In order to elucidate 
possible mechanisms underlying the TTP, we investigate two low-dimensional maps (tent map and the Hénon map), where the TTP also occurs and can be derived analytically (tent map) or deduced geometrically (Hénon map), respectively. All results are finally discussed in the last section.

\section{Transient Chaos}

As a first step we investigate the transient behavior of the systems we are dealing with. In particular we are interested in the average lifetime $\langle\mathrm{T}\rangle$ of the chaotic transients in each system. For this purpose, we created 3000 initial conditions for each model (the creation of the respective initial conditions is explained in the Supplemental Material in section B.2 on page 139.

In order to estimate the average lifetime of the chaotic transients, for each trajectory the time until self-termination of the dynamics was measured (the criteria for self-termination for each model are described in the Supplemental Material in section B.2 on page 139. By this procedure, we determined the amount of initial conditions $N_{\mathrm{Ch}}(t)$ which still exhibit chaotic dynamics at time $t$. In transient systems, this quantity typically decreases exponentially $N_{\mathrm{Ch}}(t) \approx \exp (-\kappa t)$ where $\kappa$ is denoted as the escape rate of the system [41]. The exponential decay the escape rate $\kappa$ can be extracted, which is approximately inversely proportional to the average lifetime $\langle\mathrm{T}\rangle \approx \frac{1}{\kappa}$ of the chaotic dynamics [41]. As an example, $N_{\mathrm{Ch}}(t)$ is shown in Fig. 3.5 for simulations of the Fenton-Karma model, where the time $t$ is given in spiral periods $\mathrm{T}_{\mathrm{Sp}}$. The period of a spiral wave was estimated by an average over single long living (up to ten spiral rotations) spiral waves. In the case of the Fenton-Karma model and the BOCF model, time is given in spiral rotations $\left(\mathrm{T}_{\mathrm{Sp}}\right)$, since the spiral rotation is the relevant time scale here. One spiral rotation corresponds to $\mathrm{T}_{\mathrm{Sp}} \approx 105$ time units for the Fenton-Karma model and $\mathrm{T}_{\mathrm{Sp}} \approx 350$ time units for the BOCF model, respectively. In the following, we denote the average lifetime discussed above as $\langle\mathrm{T}\rangle_{\mathrm{IC}}$ in order to make clear that this quantity is determined based on different initial conditions (IC).

By the procedure discussed above, $\langle\mathrm{T}\rangle_{\mathrm{IC}}$ was determined for each model by an exponential fit of $N_{\mathrm{Ch}}(t)$. In order to exclude effects due to possible correlations of the initial conditions (caused by the process of the creation of the initial conditions), an amount of time was discarded for the fit. The respective offsets are given in the Supplemental Material. Finally, the average lifetime $\langle\mathrm{T}\rangle_{\mathrm{IC}}$ of each system is given in Tab. 3.1 .

Table 3.1: The average lifetime $\langle\mathrm{T}\rangle_{\mathrm{IC}}$ determined by a fit of the exponential decay of $N_{\mathrm{Ch}}(t)$ for each investigated model.

\begin{tabular}{ll}
\hline Model & $\langle\mathrm{T}\rangle_{\mathrm{IC}}$ \\
\hline Fenton-Karma Model & $195.83 \pm 0.002 \mathrm{~T}_{\mathrm{Sp}}$ \\
BOCF Model & $38.65 \pm 0.006 \mathrm{~T}_{\mathrm{Sp}}$ \\
Morris-Lecar neuronal ring network & $96.41 \pm 0.10 \mathrm{~s}$ \\
Gray-Scott Model (Supplemental Material) & $2215.71 \pm 1.57$ a.u. \\
\hline
\end{tabular}




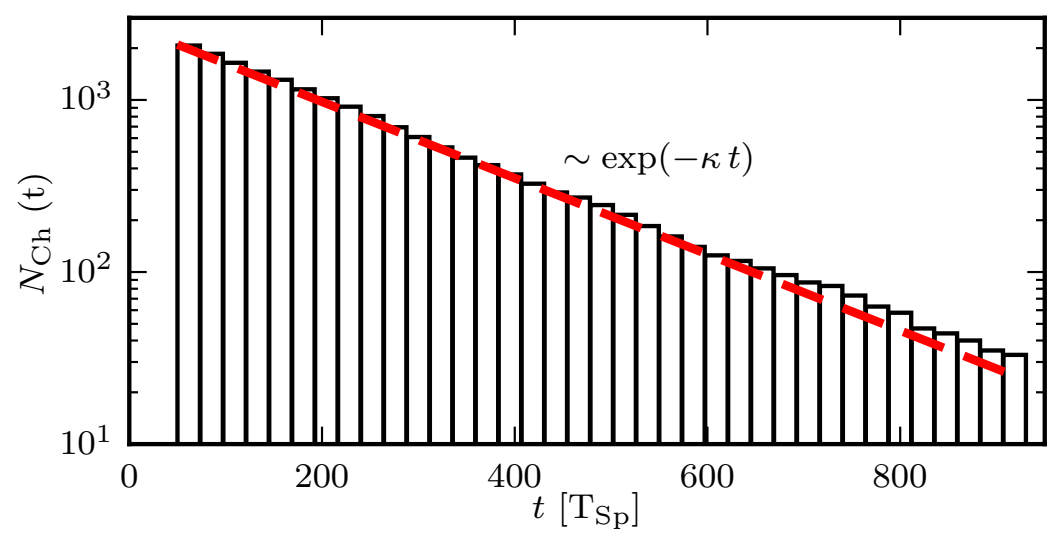

Figure 3.5: The number $N_{\mathrm{Ch}}(t)$ of initial conditions which still show chaotic dynamics at time $t$ is shown over time (in spiral periods), exemplary for the Fenton-Karma model. The exponential decay is characterized by the escape rate $\kappa$ of the system. By fitting the exponential decay ((red) dashed line) the escape rate can be extracted.

\section{The Local Vicinity of Single Trajectories}

In this section, we probe the structure of the state space concerning the lifetime of the chaotic transients. Allexandre and Otani showed, that small perturbations in the case of simulations of spiral waves can have a huge impact on the dynamics [101]. The systems are high dimensional (number of grid points of the simulation domain, times the number of dynamical variables (from $N \times N_{\text {Var }}=100 \times 2=200$ (Morris-Lecar network) up to $\left.N_{x} \times N_{y} \times N_{\text {Var }}=300 \times 300 \times 4 \approx 10^{5}(\mathrm{BOCF})\right)$. We are interested in the vicinity of single trajectories (thus, states or trajectories which are close in state space). For this purpose, we pick individual initial conditions and perturb these trajectories at specific points in time in diverse "spatial" directions. In detail, we perturb only the first dynamical variable in each model ( $u$ in the case of the Fenton-Karma model and the BOCF model, $V_{i}$ in the case of the Morris-Lecar network and $a_{i}$ in the case of the Gray-Scott model (Supplemental Material)). In the latter case, the variable we perturb represents the concentration of the first chemical specie, whereas in the other cases we modify the membrane potential. Technically, perturbing the other variables is also possible, however, we concentrate in this study on the mentioned variables, since they are (in practice) the most accessible quantities.

In the two-dimensional simulations, perturbations were applied at specific positions which are located on a coarse grained grid size of $50 \times 50$. Figure 3.6 depicts the positions of the perturbations with white dots for exemplary snapshots of the Fenton-Karma model (a) and the BOCF model (b), respectively.

In the case of the Fenton-Karma model, we applied local stimuli at a single pixel, whereas for simulations of the Bueno-Orovio-Cherry-Fenton model $2 \times 2$ pixels were perturbed. In the case of the Morris-Lecar network and the Gray-Scott model, perturbations were applied at each single node. We applied finite perturbations of various strengths. With this method, we do not focus on the local approach using linearized equations, but we can look at a small but finite vicinity of the trajectories, similar to Menck et al. [102]. It is noteworthy that 
(a)

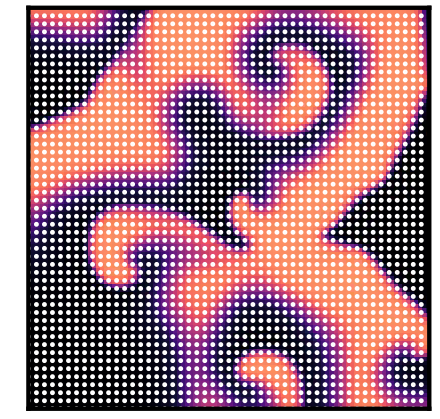

(b)

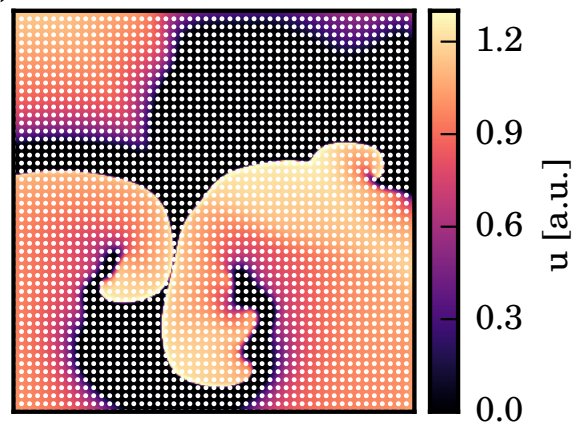

Figure 3.6: Outline of the application of the perturbations, added at specific points in time to the initial trajectory. In (a) and (b), exemplary snapshots of the Fenton-Karma model and the BOCF model, respectively, are shown. The white dots indicate the positions of possible perturbations. Only one perturbation at once was applied to the system.

only one perturbation at a time was applied, thus, by this procedure a new trajectory is created by every single perturbation. For these new trajectories the transient lifetime was determined, beginning with the point in time when the perturbation was applied.

In Fig. 3.7 a sketch of the procedure is shown: The initial trajectory (solid black line) reaches the desired attractor (red cross) at some point. Before, small perturbations are applied to the trajectory (blue arrows), which lead to new trajectories (dashed gray lines). These perturbed trajectories also reach the desired attractor, but require distinct lifetimes (the new lifetimes of the perturbed trajectories are measured from the moment of the perturbation). That means, if we apply the perturbations and measure the lifetime corresponding to the new trajectory, we can assign a transient lifetime to every perturbation (perturbed trajectory), thus to the specific position where the perturbation was applied.

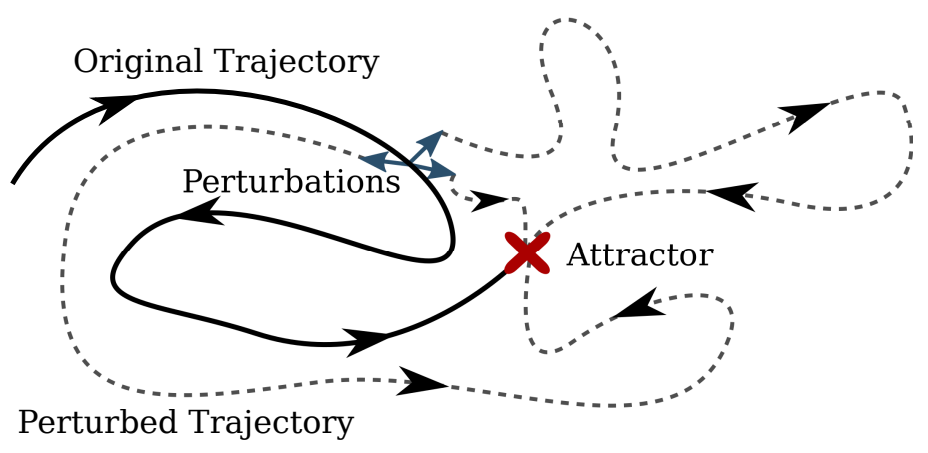

Figure 3.7: Schematic sketch of the procedure of the application of small perturbations: The initial condition (solid black line) reaches the desired attractor (red cross) without any perturbations. At some point in time, small perturbations (small blue arrows) are applied to the initial condition. This leads to new perturbed trajectories, which also reach the attractor.

As a first example, we choose a typical trajectory for each model, respectively, and apply the perturbations discussed above at a random point in time which is, however, far from 
the self-termination of the system (this case will be discussed in the following sections).

In Fig. 3.8 snapshots of the respective first variables are shown at the point in time, when the perturbations were applied (Fenton-Karma model (a), Bueno-Orovio-Cherry-Fenton model (c), Morris-Lecar network (black curve in (e))). In addition, a representation of the transient lifetimes of the trajectories which belong to perturbations applied at the corresponding location is depicted (Fenton-Karma model (b), Bueno-Orovio-Cherry-Fenton model (d), Morris-Lecar network (blue curve in (e))). In the case of the Morris-Lecar network, the strength of the perturbation is $\Delta=1.0 \mathrm{mV}$, whereas in the other cases it is $\Delta=0.1$ a.u., respectively, in order to achieve a comparable perturbation to signal amplitude ratio.

The distributions of the transient lifetimes of the perturbed trajectories $\mathrm{T}_{\text {Pert }}$ show a random structure, without any clustering or correlation to the actual state of the original system. However, the mean lifetime averaged over the new "perturbed" trajectories $\langle\mathrm{T}\rangle_{\text {Pert }}$, corresponds approximately to the quantity derived in the previous section for the average lifetime $\langle\mathrm{T}\rangle_{\mathrm{IC}}$ based on different independent initial conditions (e.g. Fenton-Karma model: $\langle\mathrm{T}\rangle_{\text {Pert }}=213.37 \mathrm{~T}_{\mathrm{Sp}}$ ). Since the perturbations were applied at a point in time far away from the collapse of the system, due to the chaotic features of the dynamics the perturbed trajectories depart from each other exponentially fast and distribute over the whole state space. That is, why the quantity $\langle\mathrm{T}\rangle_{\text {Pert }}$ provides similar results as $\langle\mathrm{T}\rangle_{\mathrm{IC}}$, if the perturbations are applied far from the collapse.

\section{Transient Lifetime Distributions before self-termination}

In the previous section, exemplary trajectories were perturbed, at points in time which are far away from self-termination of the unperturbed dynamics. Here we want to investigate the impact of perturbations applied close to the collapse of the system. For this purpose, the system was perturbed at equidistant time intervals before the collapse.

Figures 3.9 and 3.10 present the analysis of this study for the Fenton-Karma model and the Bueno-Orovio-Cherry-Fenton model, respectively. For the respective subplots (a), a representative trajectory was chosen. The $\mathrm{x}$-axis represents the time (in spiral periods $\mathrm{T}_{\mathrm{Sp}}$ ) and the black arrow indicates the moment when the chaotic dynamics of the unperturbed trajectory terminates. Perturbations of various strengths (0.001, 0.01, 0.1 and 1.0) were applied before this time as described in the previous section and the mean lifetime averaged over the new "perturbed" trajectories $\left(\langle T\rangle_{\text {Pert }}=\sum_{\mathrm{k}} \mathrm{T}_{\mathrm{k}}\right.$, where $\mathrm{k}$ runs over all perturbed trajectories) was calculated (e.g. $50 \times 50=2500$ in the case of the Fenton-Karma model). Since we want to investigate the vicinity of single trajectories and in particular the lifetime of trajectories close to the original one, $T_{k}$ is measured, starting from the point in time, when the perturbation was applied. At times which are sufficiently far away from the collapse of the system, the transient lifetimes averaged over perturbed trajectories $\langle\mathrm{T}\rangle_{\text {Pert }}$ are approximately equal to $\langle\mathrm{T}\rangle_{\mathrm{IC}}$, calculated in the previous sections. Still, fluctuations of $\langle\mathrm{T}\rangle_{\text {Pert }}$ from $\langle\mathrm{T}\rangle_{\text {IC }}$ can appear for single trajectories (decrease of $\langle\mathrm{T}\rangle_{\text {Pert }}$ in Fig. 3.10(a) at around $t=65 \mathrm{~T}_{\mathrm{Sp}}$ ).

At times closer to the collapse, $\langle\mathrm{T}\rangle_{\text {Pert }}$ drops, indicating that a decreasing amount of per- 
(a)

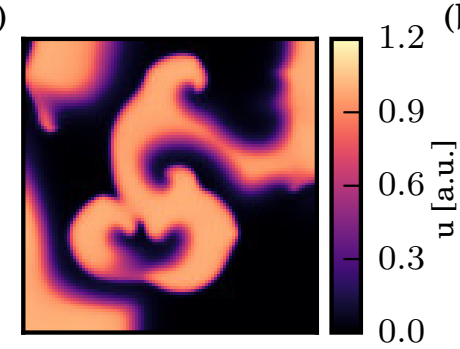

(b)

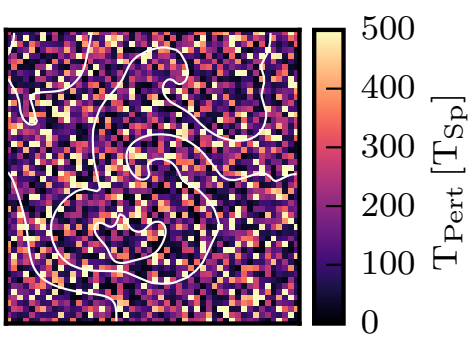

(c)
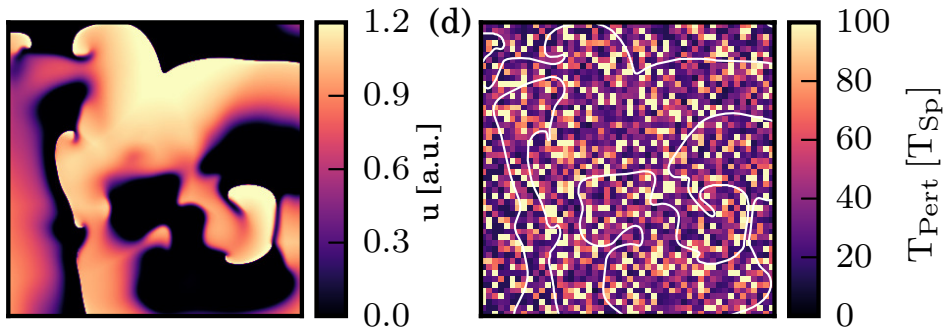

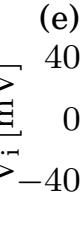

(e)

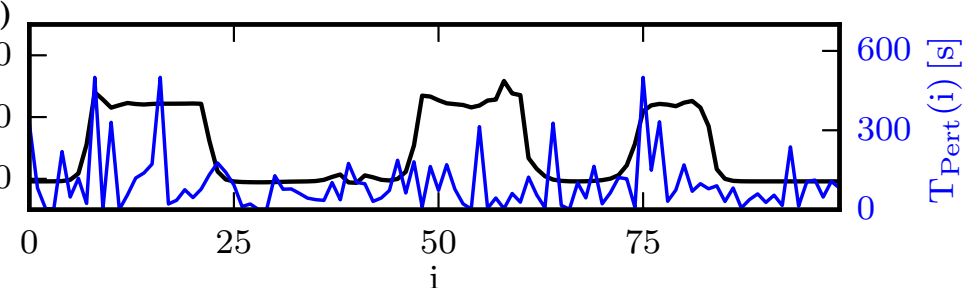

Figure 3.8: The impact of the perturbations applied to typical trajectories. The snapshots of the first variable at the point in time when the perturbations were applied are depicted in (a) for the Fenton-Karma model, (c) for the Bueno-Orovio-Cherry-Fenton model and as the black curve in (e) for the Morris-Lecar network, respectively. A perturbation applied at a specific position results in a new trajectory with its own transient lifetime. This lifetime $T_{\text {Pert }}$ corresponding to the position of the perturbation is plotted color-coded for the FentonKarma model in subplot (b) and for the BOCF model in subplot (d). In both cases, solid white curves represent the contour of $u=0.5$ of the corresponding snapshots in (a) and (b). In subplot (e), $\mathrm{T}_{\text {Pert }}$ is shown as the blue curve, for the Morris-Lecar network. In general, the transient lifetimes do not seem to show any correlation with the structure of the state at which the perturbations were applied. 

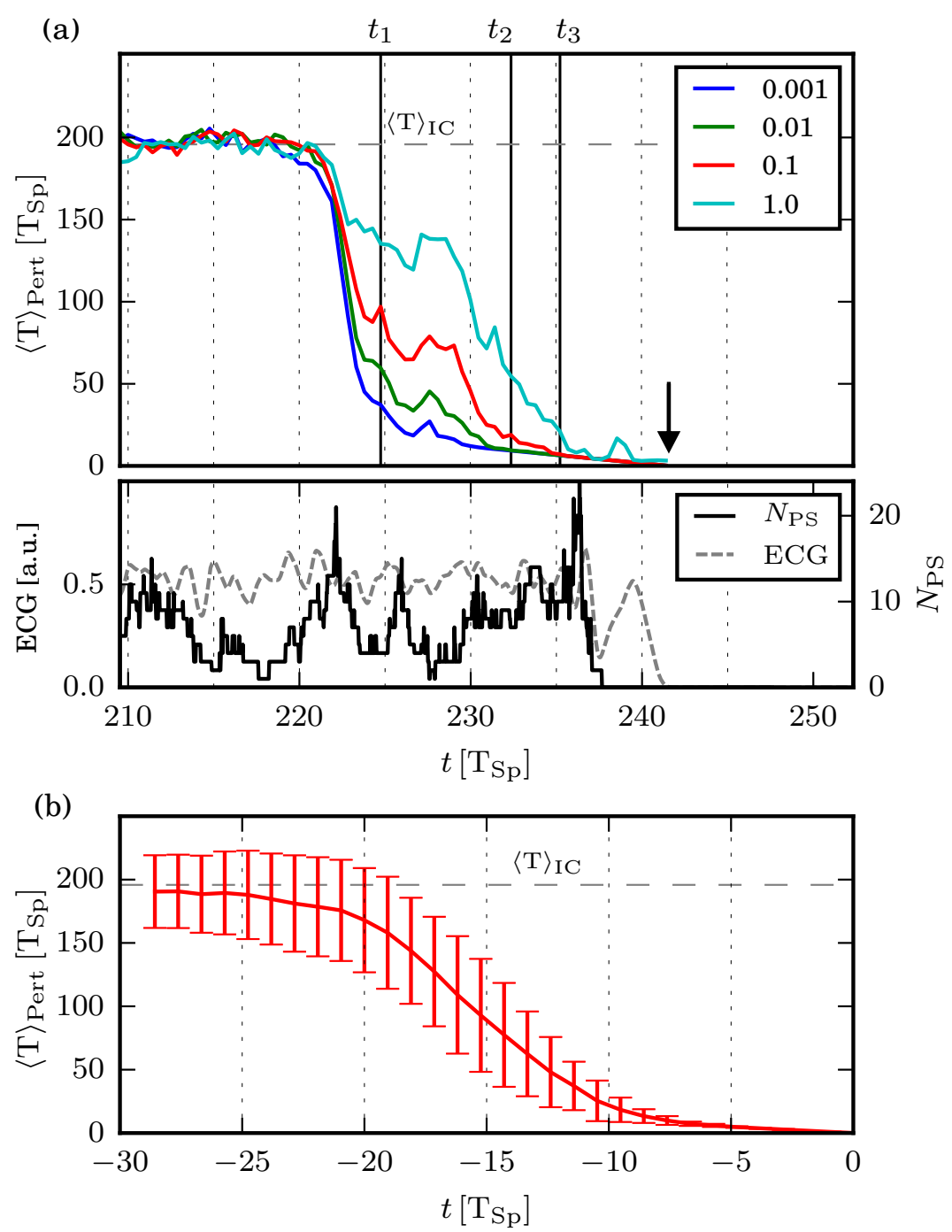

Figure 3.9: Temporal correlation of $\langle\mathrm{T}\rangle_{\text {Pert }}$ and the self-termination for the FentonKarma model. In subplot (a), a single trajectory was chosen, which terminates at around $t=$ $242 \mathrm{~T}_{\mathrm{Sp}}$ (black arrow). $\langle\mathrm{T}\rangle_{\text {Pert }}$ is plotted for various perturbation strengths (in color: [0.001, $0.01,0.1,1.00]$ ). It drops (differently for each strength) already at a finite amount of time before the actual collapse of the system, indicating that fewer perturbations can actually change the evolution with respect to the unperturbed trajectory, significantly. However, stronger perturbations have a better chance of preventing the upcoming collapse. The selftermination is not visible in the pseudo Electrocardiogram (ECG, dashed gray line) or the number of phase singularities ( $N_{\mathrm{PS}}$, solid black line) only instantly before the collapse, shown in the lower part of (a). For a perturbation strength of $\Delta=0.1,\langle\mathrm{~T}\rangle_{\text {Pert }}$ was averaged over twenty trajectories $((\mathrm{b})$, time axis is normalized such that self-termination occurs at $t=0)$, indicating a $\mathrm{TTP}_{\mathrm{FK}} \approx 20-25 \mathrm{~T}_{\mathrm{Sp}}$. 

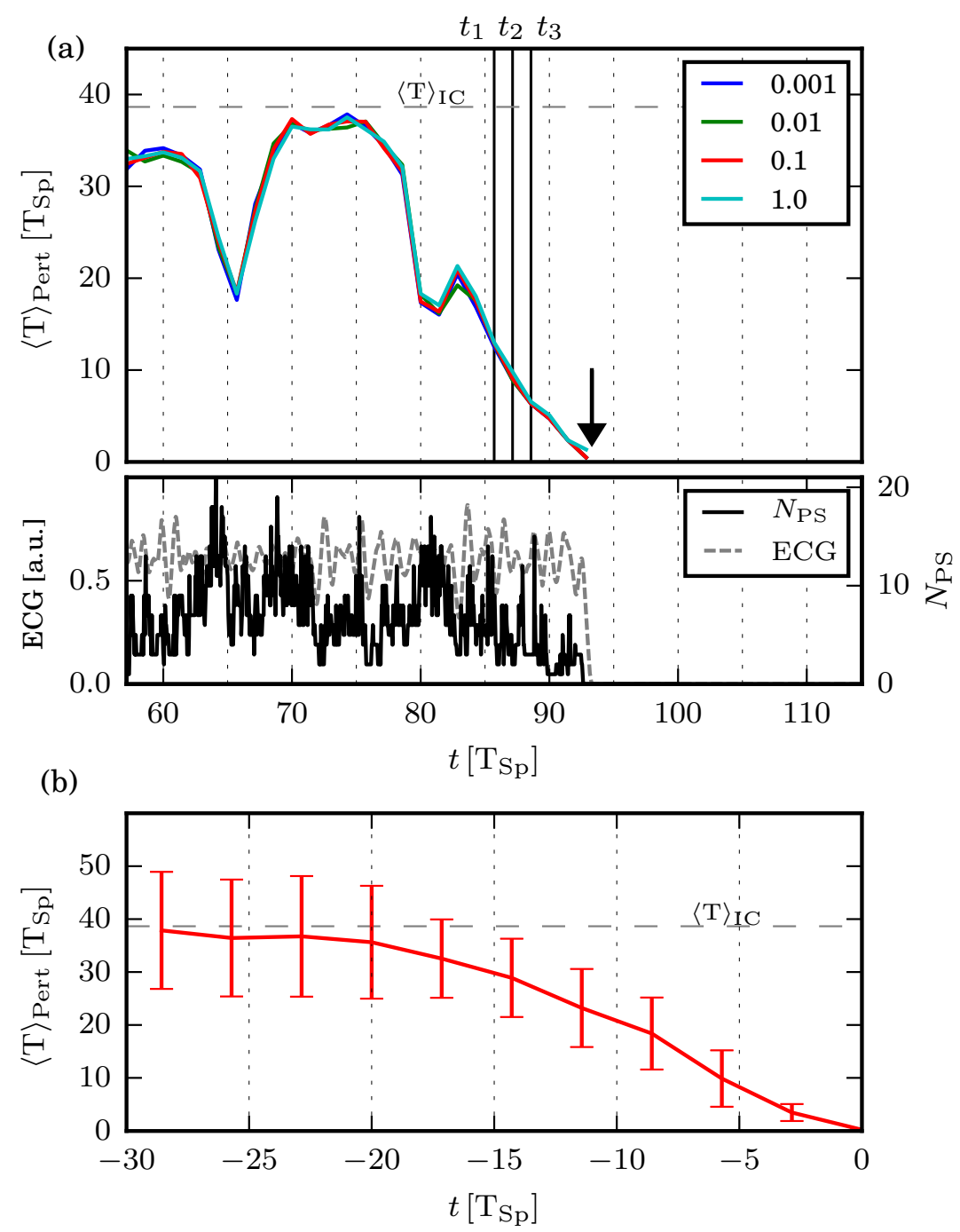

Figure 3.10: Temporal correlation of $\langle\mathrm{T}\rangle_{\text {Pert }}$ and the self-termination for the BuenoOrovio-Cherry-Fenton model. In subplot (a), a single trajectory was chosen, which terminates at around $t=93 \mathrm{~T}_{\mathrm{Sp}}$ (black arrow). $\langle\mathrm{T}\rangle_{\text {Pert }}$ is plotted for various perturbation strengths (in color: $[0.001,0.01,0.1,1.00]$ ). It drops already at a finite amount of time before the actual collapse of the system, indicating that fewer perturbations can actually change the evolution with respect to the unperturbed trajectory, significantly. In contrast to the case using the Fenton-Karma model (Fig. 3.9), perturbations of different strengths seem not to have a different impact on $\langle\mathrm{T}\rangle_{\text {Pert }}$. The self-termination is not visible in the pseudo Electrocardiogram (ECG, dashed gray line) or the number of phase singularities $\left(N_{\mathrm{PS}}\right.$, solid black line) only instantly before the collapse, shown in the lower part of (a). For a perturbation strength of $\Delta=0.1,\langle\mathrm{~T}\rangle_{\text {Pert }}$ was averaged over twenty trajectories ((b), time axis is normalized such that self-termination occurs at $t=0)$, indicating a $\mathrm{TTP}_{\mathrm{BOCF}} \approx 15-20 \mathrm{~T}_{\mathrm{Sp}}$. 
turbations is able to prevent the self-termination of the system (Figs. 3.9(a) and 3.10(a)). In Fig. 3.9.(a) (Fenton-Karma model), stronger perturbations show a higher $\langle\mathrm{T}\rangle_{\text {Pert }}$, because perturbations with a larger amplitude can shift the initial trajectory over a larger distance in the state space, which results in a higher chance of preventing the collapse. However, in the Bueno-Orovio-Cherry-Fenton model (Fig. 3.10(a)) this is not the case, and the strength of the perturbations does not seem to have an influence here.

Independently of the strength of the perturbations, in both models we can identify a finite time window before self-termination, indicating that the structure of the state space changes significantly in the vicinity of the self-termination of the trajectory (characterized by the drop of $\langle\mathrm{T}\rangle_{\text {Pert }}$ in comparison to $\left.\langle\mathrm{T}\rangle_{\mathrm{IC}}\right)$. From now on we denote this interval as the Terminal Transient Phase (TTP). An exemplary video showing the episode investigated in Fig. 3.9 (a) including the evolution of $\langle\mathrm{T}\rangle_{\text {Pert }}$ for a perturbation strength of 0.1 can be found in the Supplemental Material.

The analysis of simulations of the Morris-Lecar network is shown in Fig. 3.11 (the corresponding analysis of the Gray-Scott model is presented in Fig. B.5 of the Supplemental Material). As for the other models, a single trajectory is chosen (a), where perturbations were applied with various strengths. The influence of the strength of the perturbations seems to be moderate here.

In general, the existence of this finite amount of time, where the trajectory propagates before the collapse through a qualitatively different region of the state space (in comparison to the preceding dynamics), significantly extends the general understanding of the collapse in spatially extended systems [43, 94, 103]. Actually, the upcoming self-termination is not visible in commonly used observables like the pseudo Electrocardiogram (ECG, mean excitation integrated over the whole simulation domain: $\frac{1}{N_{x} \times N_{y}} \sum_{i, j} u_{i j}$ ) or the number of phase singularities $\left(N_{\mathrm{PS}}\right)$ (Fenton-Karma model and Bueno-Orovio-Cherry-Fenton model) shown for the first two models in the lower part of Figs. 3.9 (a) and 3.10(a), respectively. Also in the Morris-Lecar network, quantities like the mean value of the membrane potential $V_{i}$ averaged over all neurons do not provide at first sight information about the collapse of the dynamics (lower subplot in Fig. 3.11(a)).

Nevertheless, the analysis of $\langle\mathrm{T}\rangle_{\text {Pert }}$ shows that the structure of the state space already changes a finite amount of time (TTP) before the actual collapse. In order to estimate the approximate duration of the TTP, $\langle\mathrm{T}\rangle_{\text {Pert }}$ was averaged over twenty different trajectories for each model (Figs. 3.9(b), 3.10(b), 3.11(b)). In each model a perturbation strength of $\Delta=0.1$ was used except for the case of the Morris-Lecar network $(\Delta=1.0)$, in order to use a ratio of perturbation strength to signal amplitude which is of the same order of magnitude. Based on the average over twenty trajectories, an approximate value of the TTP for each model was determined (see Tab. 3.2).

In the case of the Fenton-Karma model and the BOCF model the length of the TTP is given in terms of spiral rotations. This is the main time scale of the creation and annihilation of spiral waves and thus the underlying mechanism which leads to the collapse of the dynamics. In the case of the Morris-Lecar network the length of the TTP is additionally depicted for the exemplary trajectory in Fig. 3.4(c), as a white bar in order to illustrate the length of 

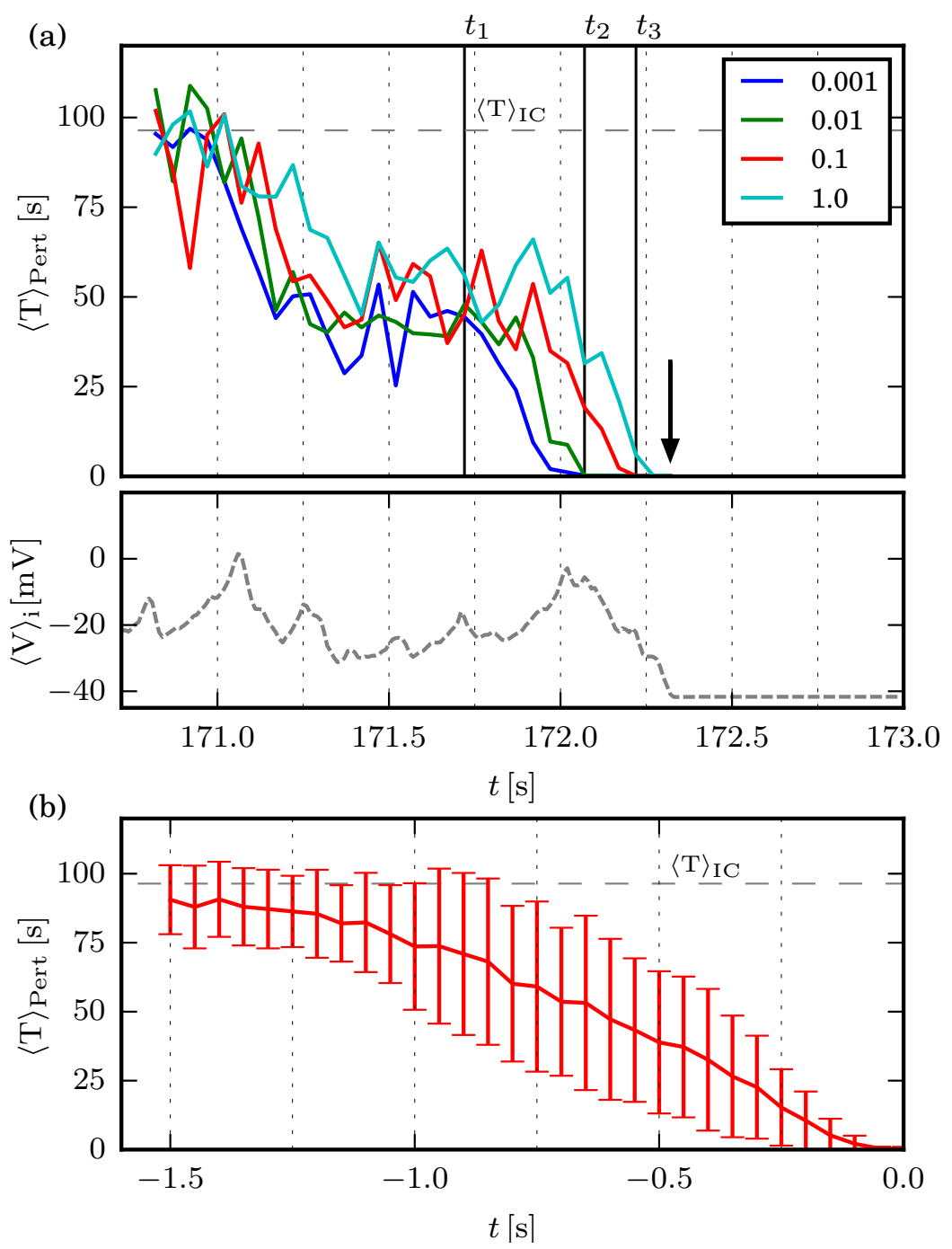

Figure 3.11: Temporal correlation of $\langle\mathrm{T}\rangle_{\text {Pert }}$ and the self-termination for the MorrisLecar network. In subplot (a), a single trajectory was chosen, which terminates at around $t=172.3 \mathrm{~s}$ (black arrow). $\langle\mathrm{T}\rangle_{\text {Pert }}$ is plotted for various perturbation strengths (in color: $[0.001,0.01,0.1,1.00])$. Varying perturbation strengths seem to have a moderate impact on $\langle\mathrm{T}\rangle_{\text {Pert }}$. The upcoming collapse is not visible in quantities like the mean (averaged over all neurons) of the membrane potential $\left(\langle V\rangle_{i}\right.$, lower subplot of (a)). For a perturbation strength of $\Delta=1.0,\langle\mathrm{~T}\rangle_{\text {Pert }}$ was averaged over twenty trajectories $((\mathrm{b})$, time axis is normalized such that self-termination occurs at $t=0)$, indicating a $\mathrm{TTP}_{\mathrm{ML}} \approx 1.5 \mathrm{~s}$. 
Table 3.2: The approximate length of the Terminal Transient Phases for each investigated model.

\begin{tabular}{ll}
\hline Model & TTP \\
\hline Fenton-Karma Model & $20-25 \mathrm{~T}_{\mathrm{Sp}}$ \\
Bueno-Orovio-Cherry-Fenton Model & $15-20 \mathrm{~T}_{\mathrm{Sp}}$ \\
Morris-Lecar neuronal ring network & $1.5 \mathrm{~s}$ \\
Gray-Scott Model (Supplemental Material) & $60 \mathrm{a} . \mathrm{u}$. \\
\hline
\end{tabular}

the TTP in relation to the actual dynamics.

\section{Spatial structure of perturbations before self-termination}

We found in the previous section the existence of a finite TTP before self-termination of the dynamics. Now we want to study, what this decrease of the transient lifetime of the perturbed trajectories distribution looks like in the state space, thus we investigate whether we find spatial structures in the space of perturbations during the TTP. For this purpose, maps of the transient lifetimes corresponding to the position of the respective perturbation (similar as in Fig. 3.8) were determined during the TTP of the examples discussed in Figs. 3.9(a), 3.10(a), 3.11(a) and B.5(a). Figure 3.12(a), (c) and (e) (FentonKarma model) as well as Fig. 3.13(a), (c) and (e) (Bueno-Orovio-Cherry-Fenton model) show the snapshots of the membrane potential $u$ at three different points in time during the TTP (additionally marked in Figs. 3.9. (a) and 3.10(a) by solid vertical lines $\left(t_{1}, t_{2}\right.$, and $\left.\left.t_{3}\right)\right)$.

Hence, the subplots in the right column of Figs. 3.12 and 3.13 show the maps of the transient lifetimes of the perturbed trajectories, for perturbations with a strength of $\Delta=0.1$. In both models, connected regions or clusters can be recognized where perturbations can significantly change the (unperturbed) reference trajectory and generate diverse transient times. In the remaining regions, perturbations can not prevent the termination of the initial dynamics. The closer the system is to the collapse, the smaller the regions with significantly different transient lifetimes become (comparison of Fig. 3.12(b), (d) and (f), and Fig. 3.13(b), (d) and (f), respectively).

The analysis of the Morris-Lecar network provides similar results: Figure 3.14 shows snapshots of the first variable (black curves in (a), (b) and (c)) at three points in time $\left(t_{1}, t_{2}\right.$ and $t_{3}$ ) which correspond to the vertical black lines in Fig. 3.11(a). In addition, the lifetimes of the perturbed trajectories $\mathrm{T}_{\text {Pert }}$ are shown in blue. Comparably to the previous results, clusters of nodes are visible (e.g. around node 65 and 85 in Fig. 3.14(a)), where perturbations can not prevent the collapse anymore. These clusters grow in time $\left(t_{1}<t_{2}<t_{3}\right)$ until only perturbations at specific nodes can prevent the system from the close collapse.

Thus, the dimensionality of the vector space of perturbations which can inhibit the collapse becomes smaller and smaller, that means, also nearby trajectories reach the attractor of the system, motivating the picture of high dimensional tube like structures which leave the chaotic regime. Comparable tube- or channel-like structures in the state space were 


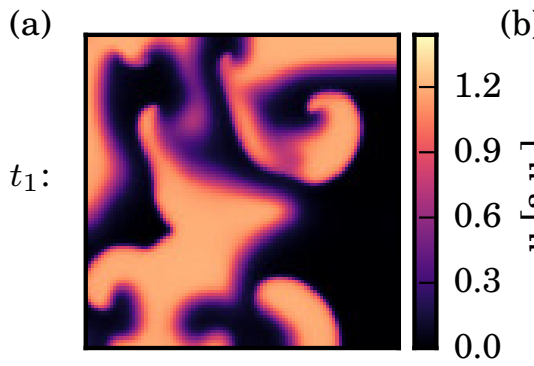

(b)

(c)

(d)
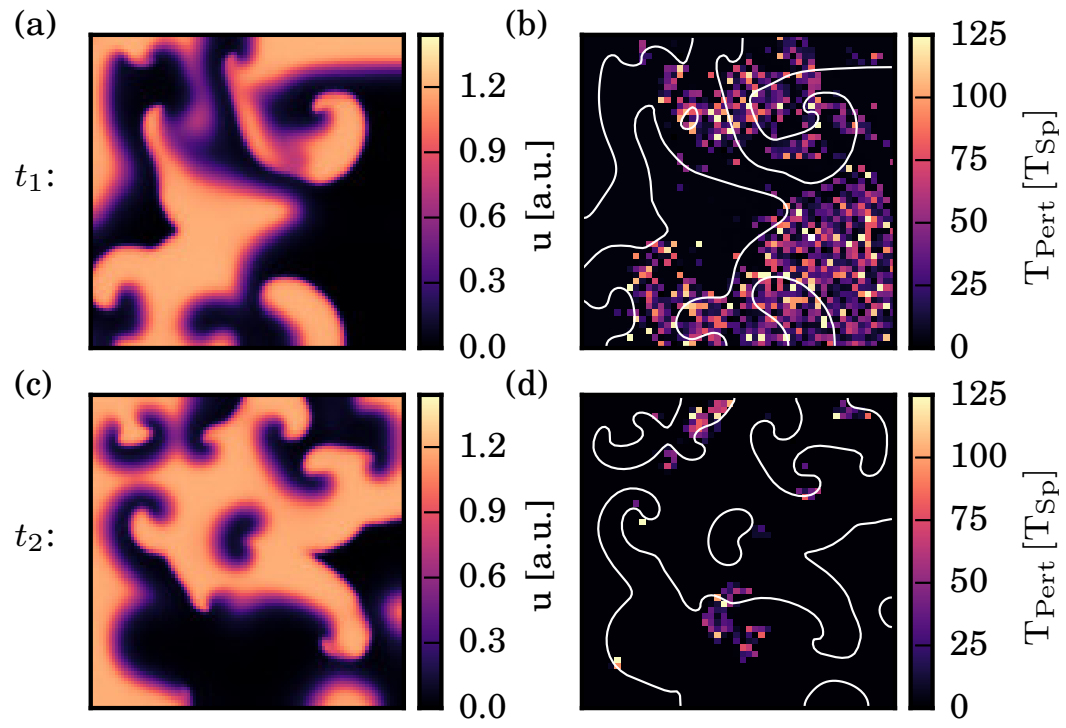

(e)

(f)
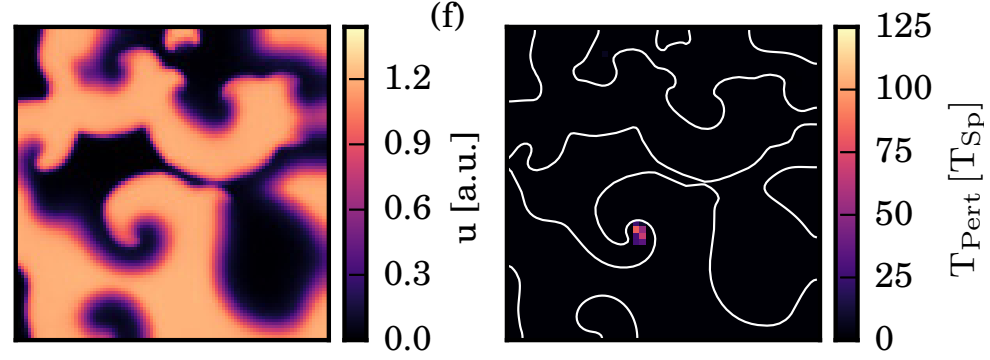

Figure 3.12: Spatial structure of the lifetime of perturbed trajectories using the FentonKarma model. The subplots (a), (c) and (e) show snapshots of the membrane potential $u$ during a TTP, at points in time which correspond to the black lines in Fig. 3.9 (a) marked by $t_{1}, t_{2}$ and $t_{3}$, respectively. The subplots (b), (d) and (e) show the map of transient times, corresponding to the trajectories which were perturbed at the specific position. The solid white curves represent the contour of $u=0.5$ of the corresponding snapshots on the left hand side. The locations of perturbations which can significantly change the transient time (with respect to the original, unperturbed trajectory) are clustered, and their number decreases as the system gets closer to the collapse of the (unperturbed) reference trajectory. 


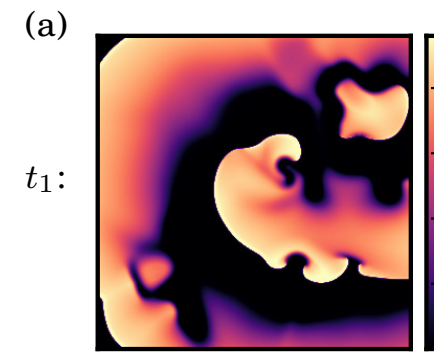

(c)

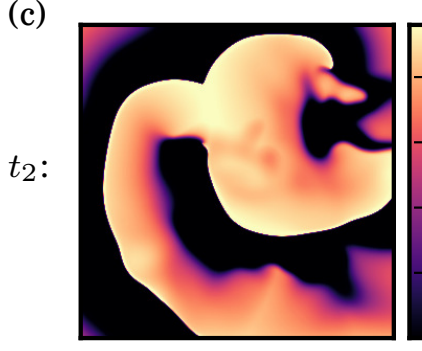

(e)

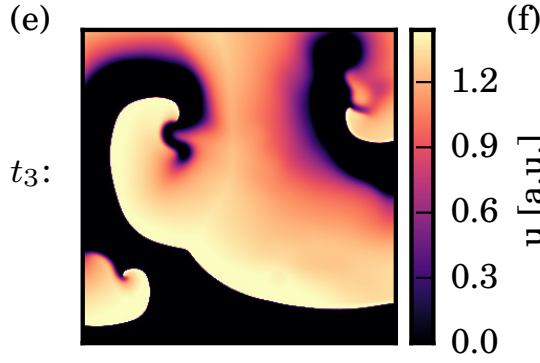

(b)

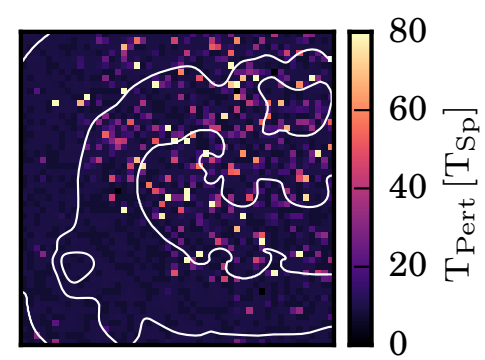

(d)

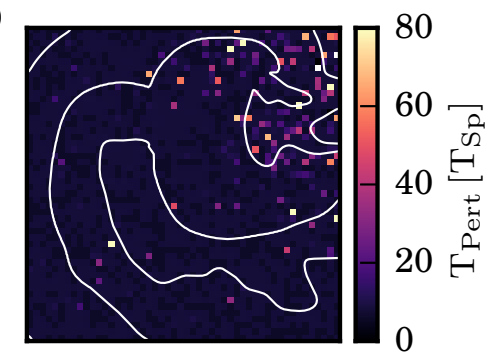

(f)

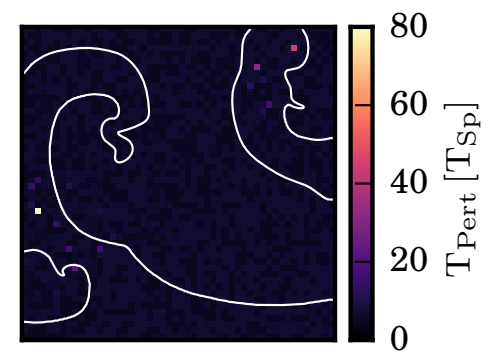

Figure 3.13: Spatial structure of the lifetime of perturbed trajectories using the BuenoOrovio-Cherry-Fenton model. The subplots (a), (c) and (e) show snapshots of the membrane potential $u$ during a TTP, at points in time which correspond to the black lines in Fig. 3.10(a) marked by $t_{1}, t_{2}$ and $t_{3}$, respectively. The subplots (b), (d) and (e) show the map of transient times, corresponding to the trajectories which were perturbed at the specific position. The solid white curves represent the contour of $u=0.5$ of the corresponding snapshots on the left hand side. The locations of perturbations which can significantly change the transient time (with respect to the original, unperturbed trajectory) are clustered, and their number decreases as the system gets closer to the collapse of the (unperturbed) reference trajectory. 

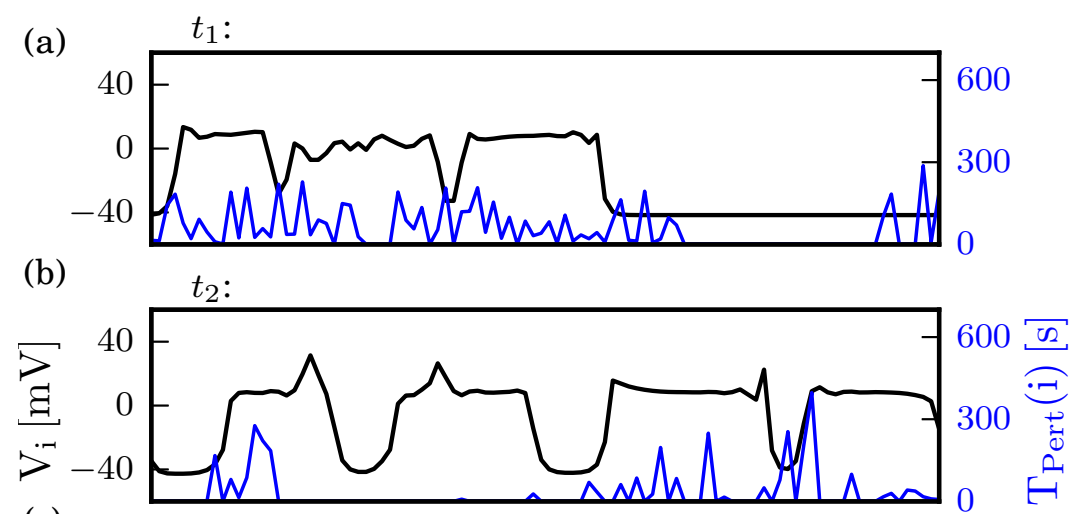

(c)

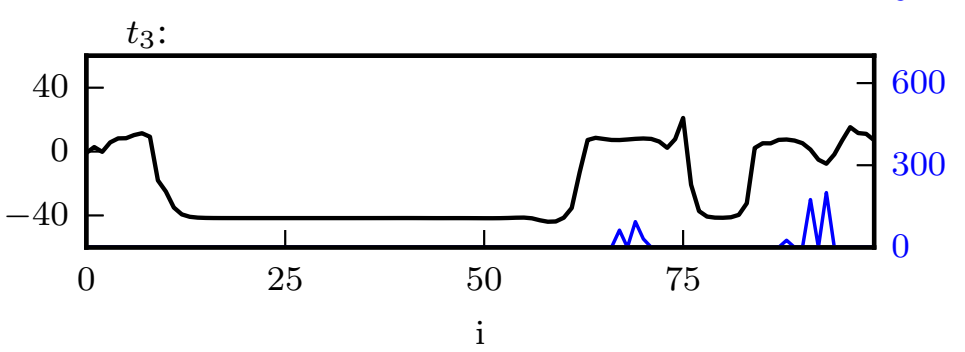

Figure 3.14: Snapshots of the first variable of the Morris-Lecar network (black) and the lifetimes $\mathrm{T}_{\text {Pert }}(\mathrm{i})$ of trajectories which resulted of a perturbation at node $i$ applied at the time the snapshot was taken (blue) concerning the exemplary trajectory discussed in Fig. 3.11(a). The subplots (a), (b) and (c) correspond to three points in time $t_{1}, t_{2}$ and $t_{3}$, respectively, which are also marked by vertical black lines in Fig. 3.11(a). Regions where perturbations can not change the average lifetime significantly (with respect to the original, unperturbed trajectory) grow in time.

also found in other systems, for example in coupled FitzHugh-Nagumo oscillators [104] or neuronal circuits [105], where in the latter case, however, the chaotic dynamics observed is "stable chaos" [106] (no positive Lyapunov exponents).

The accurate characterization and the detailed understanding of the dynamics during the TTP in the investigated systems is rather difficult, due to the huge dimensionality of the systems. That is, why in the next section, we study the underlying mechanisms of the TTP in low-dimensional maps, in order to understand the phenomenon at least in simple systems. Furthermore, this analysis provides suggestions, what the dynamics in high-dimensional systems might be.

\section{Terminal Transient Phase in Low Dimensional Maps}

We also found the TTP in low-dimensional maps, which are not comparable (or generalizable) in many aspects to the high-dimensional systems we investigated before, but still provide the advantage that possible mechanisms can be studied here in more detail. We consider the tent map and the Hénon map, which show chaotic transients in certain parameter regimes. In both maps, the transient chaotic dynamics can be achieved by a bifurcation which leads to a collapse between a former chaotic attractor and its own basin. This behavior has not been reported to our knowledge for any of the other systems discussed in this 
study, which makes a transfer of results concerning governing mechanisms difficult. However, the investigation of the Hénon map and the tent map: (i) shows that the TTP also exists in simple low-dimensional systems, (ii) provides an intuitive sketch of a mechanism potentially also underlying TTP in high dimensional systems and (iii) offers examples of what observables, which predict the collapse or give information about the structure of the state space, may look like.

\section{Tent Map}

Transient chaotic dynamics can be generated in the tent map (Eq. (3.5) by extending the value of the parameter $a$ above 1.0 and creating in this way an "exit" from the chaotic regime. Figure 3.15(a) shows a Cobweb plot for a representative initial condition $x_{0}=0.13$ and $a=2.2$. The trajectory leaves the chaotic regime after fifteen iterations through the "exit window" around $x=0.5$.

For $a=2.001$, (positive) perturbations of various strengths were applied to 1000 initial conditions. Details about the creation of the initial conditions and the definition of selftermination are given in the Supplemental Material. Comparable to the more complex systems discussed before, a drop of $\langle\mathrm{T}\rangle_{\text {Pert }}$ occurs also here at a specific number of iterations before the collapse (Fig. 3.15(c)).

For the tent map, these curves can actually be understood in a semi-analytical way: the (one dimensional) domains in state space, which lead to a collapse in $n$ iterations can be calculated. Figure 3.15(b) presents these domains (for $a=2.2$, for reasons of presentability), where a negative $n$ is used, since $n$ denotes the number of steps before the collapse (at $n=0)$. The number of the domains grows with $2^{1-n}$ and their length $L(n)$ can be calculated analytically

$$
L(n)=\left(\frac{a-2}{a}\right)^{-n}
$$

The probability that a trajectory which is located inside such a domain will be kicked out of this domain by a perturbation of strength $p$ can hence be given geometrically by the ratio of the length of the domain and the strength of the perturbation $\frac{p}{L(n)}$. In Fig. $3.15(\mathrm{~b})$ this is sketched using a representative perturbation (gray arrow). For $n=-1$, the perturbation has a small probability to shift a trajectory out of the domain (only those trajectories which are located at the right edge of the domain). In all other cases, the perturbation only moves the trajectory in between the domain, which does not prevent the collapse in $n=-1$ steps. In the case of $n=-2$, this probability has increased, since the length of the domain has decreased. The number of the domains does actually not play a role here. For $n=-3$, the perturbation is larger than the whole size of the domain, and will therefore kick all trajectories out of the domain. With these considerations, $\langle\mathrm{T}\rangle_{\text {Pert }}$ can be given analytically, depending on the number of steps before the collapse $n$ and the perturbation strength $p$ 
(a)

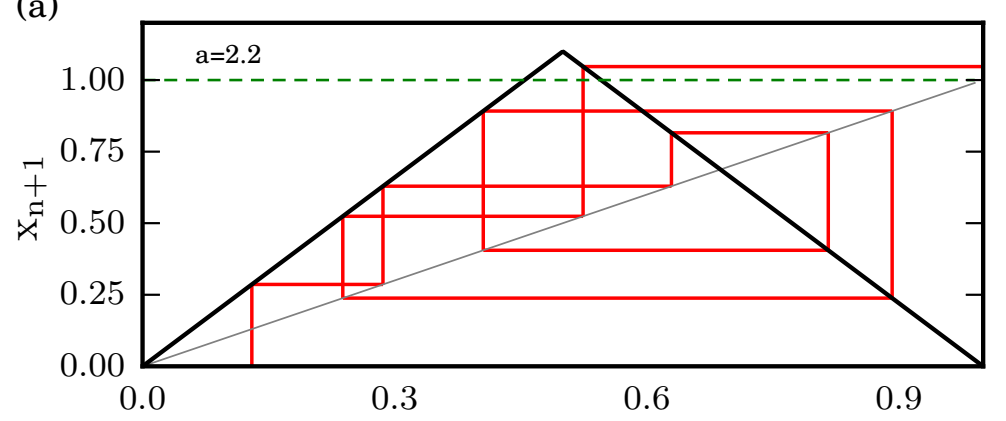

(b)

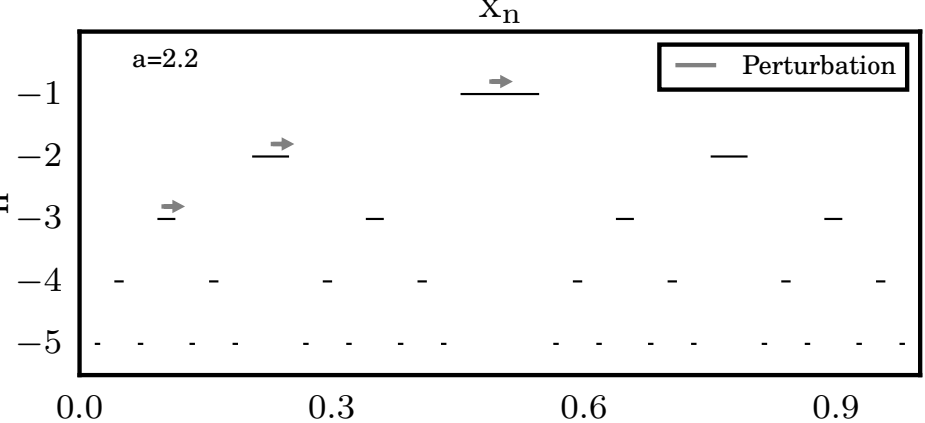

(c)

$\mathrm{x}_{\mathrm{n}}$

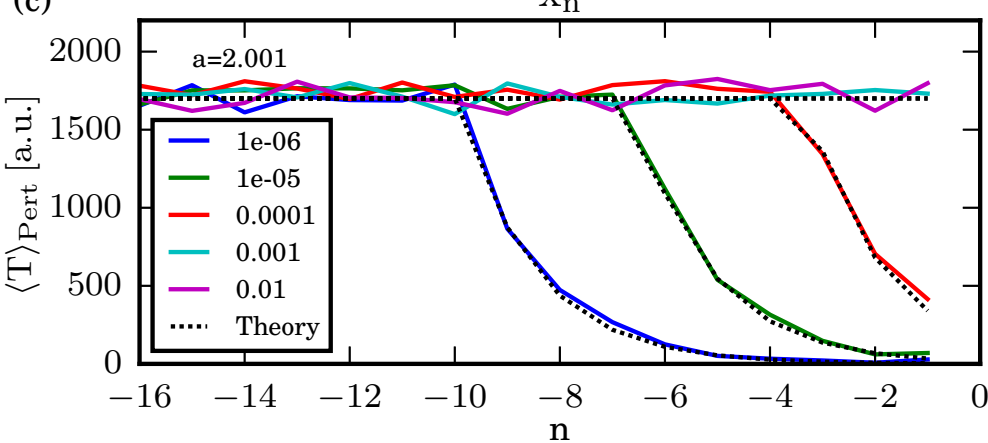

Figure 3.15: The Terminal Transient Phase in the tent map. Subplot (a) shows a Cobweb plot for a representative initial condition $x_{0}=0.13$ and $a=2.2$. In (b), the domains of the state space are shown, which will lead to self-termination in $n$ steps $(n=0$ means self-termination). For $n=-1$ the domain corresponds to the exit-like window in subplot (a) (between the two intersections of the green dashed line $\left(x_{n+1}=1\right.$ ) and the black line (tent map)). The grey arrows indicate a representative perturbation. The ratio between the perturbation strength (length of the arrow) and the domain sizes increases when departing from the collapse $(n=0)$ backward in time. In subplot $(\mathrm{c}),\langle\mathrm{T}\rangle_{\text {Pert }}$ is shown for various perturbation strengths (colored lines), and also the theoretical approximation (Eq. (3.9)) is plotted for each perturbation strength (black dotted lines). 


$$
\langle\mathrm{T}\rangle_{\text {Pert }}(n, p)=\langle\mathrm{T}\rangle_{\mathrm{IC}} \cdot \frac{p}{L(n)}
$$

where $\langle\mathrm{T}\rangle_{\mathrm{IC}}$ is the average lifetime calculated based on the lifetimes of the (unperturbed) the initial conditions. This theoretical expression is additionally plotted in Fig. 3.15(c) using black dashed lines, for each perturbation strength, respectively, and coincides well with the numerical results.

\section{Hénon Map}

The Hénon map [33] is a two-dimensional invertible map, defined by Eqs. (3.6) and (3.7) [107. Hinke M. Osinga showed, how a boundary crisis bifurcation leads to transient chaos in this system [107, where in a certain parameter regime, the chaotic attractor collides with its own boundary. Figures 3.16(a) and (b) depict such a transition. In subplot (a) for $a=1.4$, the chaotic attractor (red) can be determined by the unstable manifold of a fixed point $P_{1}$ (blue dot in (a)), whereas the basin of the attractor (green) is given by the stable manifold of a second fixed point $P_{2}$.

With a parameter value of $a=1.428$, the boundary crisis has taken place (Fig. 3.16(b)) and the unstable manifold of $P_{1}$ touches the stable manifold of $P_{2}$ at infinitely many points. Similar to the investigations before, 5000 initial conditions were homogeneously distributed inside the (former) basin and perturbations were applied. Details about the creation of the initial conditions and the definition of the self-termination can be found in Supplemental Material. The perturbations are two-dimensional $(x, y)$, in random directions, but with a constant perturbation strength. In Fig. 3.16(c) $\langle\mathrm{T}\rangle_{\text {Pert }}$ is shown for different perturbation strengths and the TTP occurs again in this model. Furthermore, we found that all trajectories are passing relatively small areas in the state space before their termination. These regions of the state space (marked by (i)-(iv) in subplot (b)) are explicitly shown in subplot(d). Trajectories pass these regions 9 (i), 8 (ii), 6 (iii) and 1 (iv) steps before self-termination, respectively (also marked as vertical dashed lines in subplot (c)). In the enlarged maps of the state space (subplot (d)), the distribution of the 5000 trajectories is depicted in black. Furthermore we found, that although the chaotic attractor does not exist anymore here, the stable manifold of the fixed point $P_{2}$ still determines the boundary between chaotic (grey shaded) and non-chaotic domains of the state space. The area colored in orange gives the region where the trajectories are distributed after a perturbation of strength 0.01 (orange curve in subplot (c)). In the previous case of the tent map, $\langle\mathrm{T}\rangle_{\text {Pert }}$ could also be determined analytically. Here, the drop of $\langle\mathrm{T}\rangle_{\text {Pert }}$ can be understood at least using an intuitive geometrical picture: The overlap of the orange area (perturbed trajectories) and the grey shaded region of the state space (chaotic regime) is a good approximation for the amount of trajectories which remain in the chaotic regime (prevention of the collapse). Using this perspective, one can explain the drop of $\langle\mathrm{T}\rangle_{\text {Pert }}$. This is in particular plausible for the case $n=-1$ ((iv), thus trajectories which are one step away from collapsing), where only few trajectories can be perturbed to the chaotic regime (corre- 


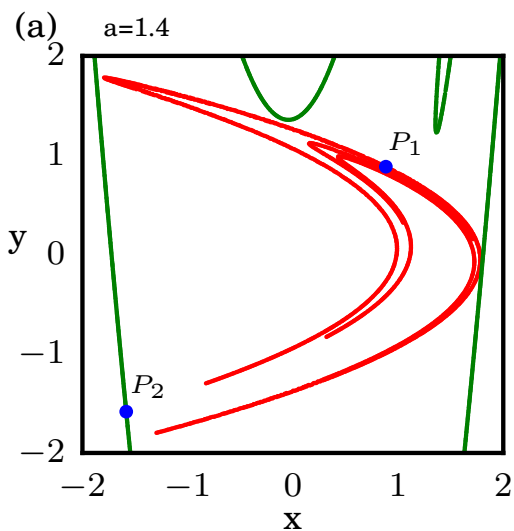

(b) $\mathrm{a}=1.428$
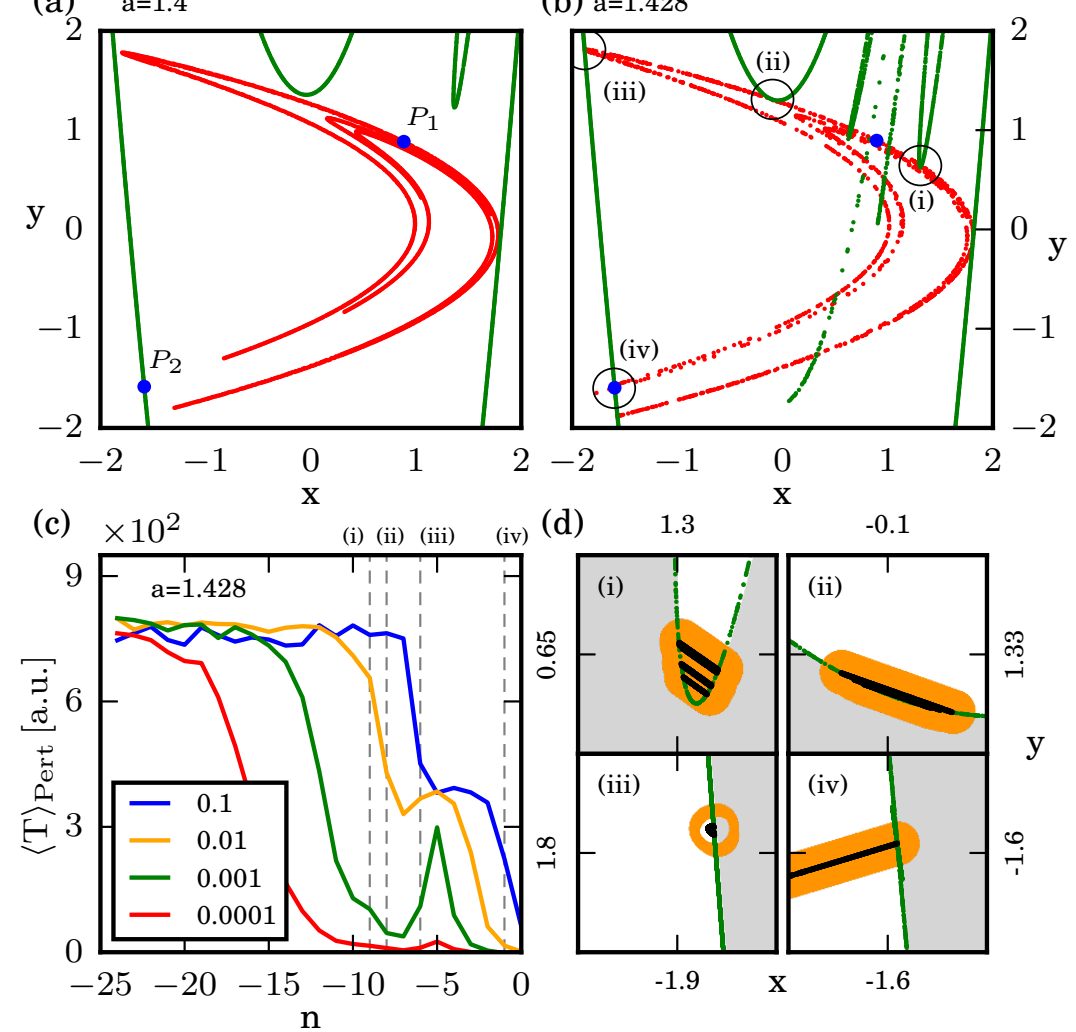

(d) $\quad 1.3$

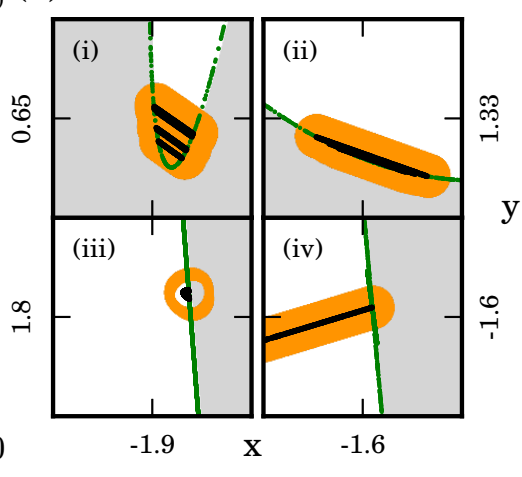

Figure 3.16: The Terminal Transient Phase in the Hénon map. Subplots (a) and (b) depict the boundary crisis bifurcation which causes the transient dynamics. In (a) $(a=1.4)$, the attractor of the system (red) is described by the unstable manifold of the fixed point $P 1$, whereas its basin is determined by the stable manifold of the fixed point $P_{2}$. In (b) $(a=1.428)$, the attractor has collided with its own basin. $\langle\mathrm{T}\rangle_{\text {Pert }}$ is shown in (c) for various perturbation strengths. Specific regions of the state space (marked by (i)-(iv) in (b)) are magnified in subplot (d), each plot with a size of $(0.1,0.1)$. Trajectories pass these regions before the self-termination (see also (c)). The distribution of the 5000 trajectories before the application of a perturbation are marked in black in subplot (d), whereas the distribution after the application of a perturbation of strength 0.01 (orange curve in (c)) is marked in orange. The grey shaded region denotes here the chaotic regime of the state space. That means, trajectories which are perturbed into the grey region stay chaotic, whereas they collapse if they are perturbed into the white region. Thus, by geometrically considerations, the overlap of the grey and the orange domains does roughly estimate the probability, that an arbitrary perturbation can prevent the trajectory from the collapse. 
sponds to $\langle\mathrm{T}\rangle_{\text {Pert }} \approx 15.8$ a.u. (orange curve in $\left.(\mathrm{c})\right)$ )). Also the difference of $\langle\mathrm{T}\rangle_{\text {Pert }}$ between different perturbation strengths at $n=-9$ ((i), thus trajectories which will collapse in nine steps) is reasonable in this picture when referring to subplot (d)(i): smaller perturbations as 0.01 (orange curve) cannot shift most of the trajectories back to the chaotic regime (grey shaded region), due to the distance of most of the trajectories (black) to the (former) basin boundary (green).

The existence of the TTP in the tent map and the Hénon map underlines the robustness of this phenomenon. The drop of $\langle\mathrm{T}\rangle_{\text {Pert }}$ can be understood analytically (tent map) or using the geometrical picture (Hénon map).

In addition, these examples show that an observable which can predict the collapse or give information about the state space exists (at least in these low-dimensional systems): in both maps we characterized domains of the state space, where trajectories are located that collapse in the near future. Knowing the location of the current state relative to these domains, we can therefore predict the collapse of the trajectory. Furthermore, the dynamics of an arbitrary trajectory can be modified by the application of perturbations, which aim at pushing the trajectory into the abovementioned domains of the state space, and thus forcing the collapse of the chaotic dynamics.

\subsubsection{Conclusion}

In numerical simulations of six systems we have shown the transient nature of chaotic dynamics in spatially extended systems and low-dimensional maps. We investigated two models which are used to simulate action potential propagation of cardiac tissue (FentonKarma model and the Bueno-Orovio-Cherry-Fenton model), a ring network of Morris-Lecar elements which models neuron activity and a one dimensional system of the Gray-Scott model which simulates a chemical reaction of two species. Furthermore, we extended our simulations to the tent map (one-dimensional) and the Hénon map (two-dimensional), which provide, due to their low dimensionality, a more direct access.

We determined the average transient lifetime $\langle\mathrm{T}\rangle_{\mathrm{IC}}$ of the dynamics by a large number of initial conditions for each model. Since this is a quantity based on the whole chaotic regime of the state space, we investigated (the vicinity of) single trajectories by small but finite perturbations in order to probe the local structure of the state space. In each model we could identify a Terminal Transient Phase (TTP) characterized by a decreasing transient

lifetime averaged over all perturbed trajectories $\langle\mathrm{T}\rangle_{\text {Pert }}$. This indicates, that trajectories propagate before self-termination through a qualitatively different (concerning the preceding dynamics) region of the state space, although this is not visible in "common" observables (e.g. the pseudo ECG or the number of phase singularities). The typical duration for trajectories to travel through this "transition zone" is significant with respect to the intrinsic time scale governing the dynamics (e.g the length of a spiral rotation) $\left(\mathrm{TTP}_{\mathrm{FK}} \approx 20-25 \mathrm{~T}_{\mathrm{Sp}}\right.$ (Fenton-Karma model), $\mathrm{TTP}_{\mathrm{BOCF}} \approx 15-20 \mathrm{~T}_{\mathrm{Sp}}$ (Bueno-Orovio-Cherry-Fenton model), $\mathrm{TTP}_{\mathrm{ML}} \approx 1.5 \mathrm{~s}$ (Morris-Lecar network) and $\mathrm{TTP}_{\mathrm{GS}} \approx 60 \mathrm{a} . \mathrm{u}$. (Gray-Scott model)). Furthermore we showed, that the drop of $\langle\mathrm{T}\rangle_{\text {Pert }}$ is related to spatial clusters of perturbations 
which do not change the initial trajectory anymore, indicating that if the system is close to the collapse, only perturbations into specific directions can prevent the system from selftermination. Simulations of the tent map and the Hénon map provide an intuitive insight into the underlying mechanism in low-dimensional systems. We could determine the drop of $\langle\mathrm{T}\rangle_{\text {Pert }}$ analytically for the tent map and found a geometrical representation in the case of the Hénon map. This provides an idea of what the dynamics and the state space may look like during the TTP in high-dimensional systems.

With this study we investigated the temporal and spatial structure (concerning the lifetime) of the transition from a chaotic regime to the (non-chaotic) attractor of the system. It is noteworthy to emphasize that the transition from the chaotic regime to the attractor can not be understood as the entering of the basin of attraction of the attractor. Typical trajectories are the whole time located inside the basin of attraction, since they will definitely reach the attractor at some point. However, since the information of the collapse is already present in the system some time before upcoming the collapse (TTP), it should be possible, in principle, to identify an observable precursor for predicting temporally close self-termination (despite the fact that type-II-supertransients are characterized by an abrupt termination). In the low-dimensional systems this can already be done (e.g. determining the (former) basin of attraction in the case of the Hénon map). To devise suitable precursors of the collapse in high-dimensional systems one might employ data assimilation or machine learning methods, to exploit the fact that during the TTP trajectories are (partially) robust with respect to small perturbations.

The Terminal Transient Phase occurs in each model investigated here, low-dimensional maps, spatially extended one- and two-dimensional systems, with no-flux or periodic boundary conditions and one or two diffusive variables. In the future, it may be of interest whether a finite TTP and corresponding clustering effects in the perturbation space also exist in other systems (e.g. fluid dynamics [36]).

The existence of a TTP extends the general understanding of type-II-supertransients and also may open up new possibilities for applications. For example, in cardiac dynamics where occurring arrhythmias can be life-threatening and the standard defibrillation technique comes along with severe side effects like additional tissue damage and considerable pain [18, 108, 19]. Since self-termination of such arrhythmias like ventricular fibrillation has been observed frequently [109], an observable precursor could improve the control of such arrhythmias. Possible applications could be heading in two directions: (i) a prediction of a close self-termination of e.g. ventricular fibrillation could prevent the submission of a defibrillation shock at all and reduce in this way the side-effects, and (ii) the energy of electrical pulses applied for defibrillation could be reduced by exploiting the state space structure. Either by detecting states where successful defibrillation can be achieved using a lower energy, or using a two step protocol and first reaching these states before defibrillating. Last but not least, transient dynamics also play a role in the functionality of the brain (e.g. information processing) [110, and thus studying these processes could benefit from taking the Terminal Transient Phase into account. 


\section{Summary of Results}

In this study, we reveal the existence of a transition zone in the state space between the chaotic dynamics and the non-chaotic attractor, that means before the self-termination of the dynamics, called the Terminal Transient Phase (TTP). We show in each of the six investigated models, that the region of the state space which corresponds to the TTP has a qualitatively different structure than the state space region which is governed by the previous chaotic dynamics. That means, the state space before the collapse of the chaotic dynamics is different then before, (Fig. 3.17), and it should therefore in principle be possible to develop proper observables which can predict the upcoming self-termination.

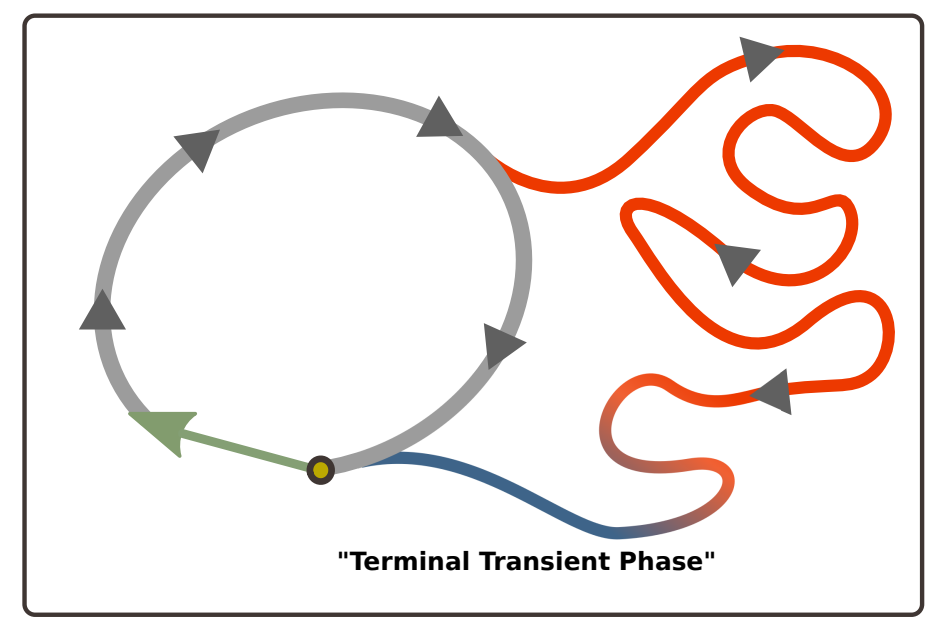

Figure 3.17: The (schematic) state space, depicting the conclusion of the study "Terminal Transient Phase of Chaotic Transients". The difference of the state space structure between the Terminal Transient Phase (TTP) and the chaotic dynamics before the TTP is illustrated here by the red and blue color of an exemplary trajectory.

Since the TTP occurs in systems with very distinct dynamics, we believe that it is a general feature of transient dynamics. Hence, the existence of the transition zone significantly extends the general understanding of chaotic transients in the field of nonlinear dynamics. Furthermore, the obtained results can also be relevant for possible applications, e.g. in the field of cardiac dynamics. An extended discussion of the results, including possible implications for applications can be found in the section "Discussion and Outlook" $4.1 \mathrm{on}$ page 125. 


\subsection{Complexity Fluctuations during Ventricular Fibrillation}

\section{Status of the manuscript}

This manuscript is currently under revision.

\section{Author Contributions}

Thomas Lilienkamp and Alexander Schlemmer contributed equally to this work.

- Thomas Lilienkamp designed the research, performed three dimensional numerical simulations in a realistic geometry, analyzed the data and wrote the manuscript.

- Alexander Schlemmer designed the research, analyzed the data and wrote the paper manuscript.

- Sebastian Berg performed two-dimensional simulations, analyzed the data and wrote the paper.

- Stefan Luther designed the research and wrote the paper.

- Daniel Hornung performed experiments and wrote the paper.

- Tariq Baig performed experiments and wrote the paper.

- Laura Diaz Rodriguez built an experimental eight-electrode setup.

- Edda Boccia performed two-dimensional simulations using the Luo-Rudy model.

- Claudia Richter performed experiments.

- Rabea Hinkel performed experiments and provided medical counseling.

- Jan Christoph provided a numerical algorithm to identify phase singularities and filaments from excitation data.

- Christian Kupatt provided medical counseling.

- Ulrich Parlitz designed the research and wrote the paper.

\section{Scientific Objective}

In the previous study, the process of self-termination of (transient) episodes of chaotic dynamics in spatially-extended systems was investigated. It was shown, that the state space of the terminal phase before the collapse of the dynamics has a qualitatively different structure then the previous chaotic dynamics.

In this study, we focus on the chaotic episode itself, excluding the final phase before selftermination. The experimental observation was made, that during an episode of ventricular fibrillation the irregularity of the ECG signal was not homogeneous but fluctuated in time. This feature motivated the question, whether these fluctuations are related to variations 
of the spatio-temporal dynamics of the chaotic electrical wave patterns. The scientific objective of this study is, whether the level of complexity of the spatio-temporal dynamics during ventricular fibrillation is constant or whether it fluctuates (Fig. 3.18).

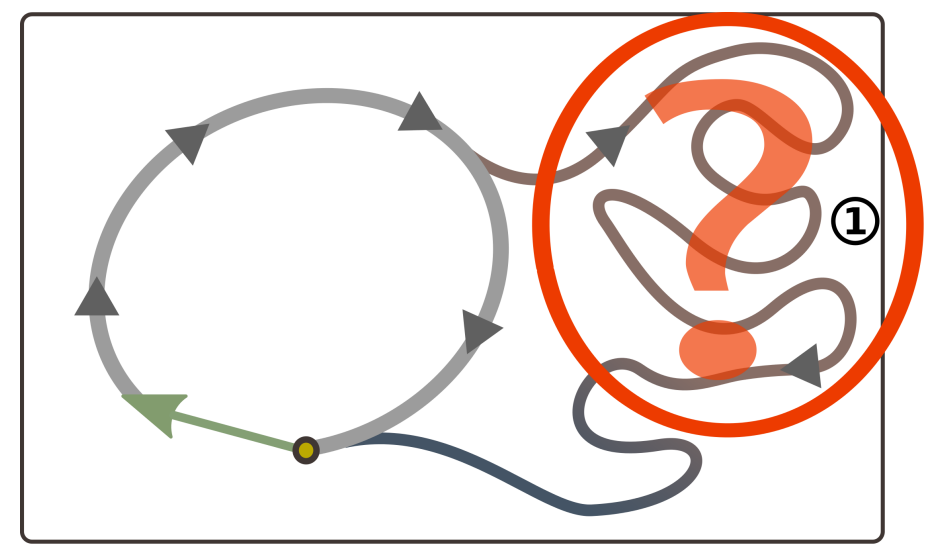

Figure 3.18: The (schematic) state space, formulating the scientific objective concerning the study "Unveiling Spatio-Temporal Complexity Fluctuations in Cardiac Arrhythmia Using Permutation Entropy of ECG Time Series". Here, we focus on the dynamics during the chaotic episode. In particular, we study the level of complexity of the spatio-temporal dynamics, and investigate whether measurable fluctuations of the organization of the scroll wave dynamics can be verified.

In three-dimensional simulations using a realistic rabbit heart geometry, the availability of the full information in numerical simulations is exploited in order to investigate the correlation between the spatio-temporal dynamics and ECG time series.

\section{Numerical Models}

Numerical simulations have been performed on a realistic rabbit heart geometry, obtained from a computed tomography scan (CT-scan). The use of a realistic heart geometry is essential for this study, since the geometrical arrangement of ECG electrodes in relation to the positions of the scroll waves is essential for the reproduction of realistic ECG signals. Also, anatomical properties of the heart (e.g. the difference in thickness between the right and left ventricular wall) are taken into account, which may have an influence on the dynamics of the scroll waves.

For the implementation of the Phase Field method (2.4.4 on page 57), Eqs. 2.69) and 2.70) have been used with $d t=0.1, h=0.5$ and $\xi=0.5$. The Fenton-Karma model was used for modeling the ion channel dynamics, with the parameter set FK3 (Tab. A.4) and $D=0.9$, $h=2.0, d t=0.1$. Since this study does not need to e.g. adapt specific ion concentrations, the Fenton-Karma model is a reasonable estimate.

Furthermore, the governing mechanism was also reproduced in two-dimensional simulations of the Fenton-Karma model (performed by Sebastian Berg) and the Luo-Rudy model [11]] (performed by Edda Boccia).

The Supplemental Material to this study can be found in section B.3 on page 147 . 


\begin{abstract}
During cardiac arrhythmias, self-organized spatio-temporal complexity in the heart is associated with the dynamics of vortex-like rotating waves and their nonlinear interaction with each other and with the heterogeneous multicellular substrate. However, the detailed mechanisms underlying the onset and perpetuation of cardiac arrhythmias remain largely elusive. Here we show that characteristic intermittent fluctuations in spatio-temporal complexity during cardiac arrhythmia can be deduced from electrocardiogram (ECG) time series using permutation entropy. Using (multiple) ECG time series of different species, optical mapping data from Langendorff-perfused intact rabbit hearts, and numerical simulations, we demonstrate that intermittency of complexity fluctuations of the electrocardiogram are associated with characteristic density fluctuations of phase singularities and filaments, being the organizing centers of fibrillation. Our findings elucidate the role of electrode position for a correct detection and may open the path for future applications for an effective classification and improved defibrillation of cardiac arrhythmias.
\end{abstract}

\title{
3.3.1 Introduction
}

Ventricular fibrillation $(\mathrm{VF})$ is a dangerous arrhythmia which leads to death after short periods of time. Reentrant electrical activity [112] causes the muscle to contract at a much higher frequency, more irregularly and therefore less efficiently than during sinus rhythm. The state of the heart is most prevalently observed using electrocardiograms (ECGs) which measure a projection of the electrical activity of the heart using electrodes placed on the skin. During normal heart beat, the ECG signal shows very distinct periodic patterns known as QRS complexes while during VF a very irregular aperiodic signal appears (see next section for examples). During VF the time series shows patterns at different temporal scales which makes a clear definition of distinct "states" difficult. One perspective that has long been known [113] differentiates between "coarse VF" and "fine VF". Coarse VF is defined [114] as having an amplitude greater than $0.2 \mathrm{mV}$.

The following observations have frequently been associated with the notion of coarse and fine VF [115]: coarse VF seems to be easier to be defibrillated, which manifests itself in a higher success rate. After a prolonged VF episode, fine VF seems to be much more prevalent than in the beginning.

Already in the early studies of coarse and fine VF the relation between these fluctuations and the electrical activity have been discussed. For example, in [115] the relation to synchronization of myocardial activation was investigated which lead to the result that coarse $\mathrm{VF}$ is not linked to a lower standard deviation in activation times.

Relying solely on the amplitude of the ECG can be expected to cause many problems, including a strong dependency on the subject [114]. Therefore many different methods to analyze the VF waveform have been introduced. For example, wavelet transforms have been suggested in [116] to quantify the temporal development of patterns in the ECG of a VF which reveal structures characteristic for different time scales.

In this article we demonstrate in a first step the application of permutation entropy (PE) 


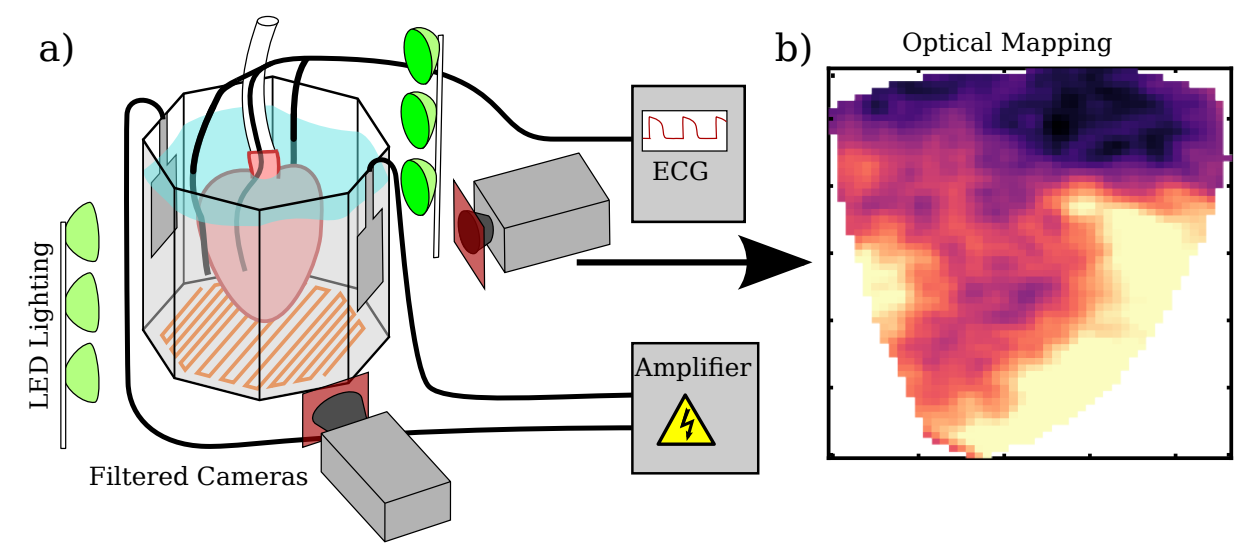

Figure 3.19: A sketch of the experimental setup showing the heart in the perfusion system with an attached ECG electrode (a). The cameras record simultaneously videos of the excitation patterns which are made visible using voltage sensitive dye. A snapshot of such a video is shown in (b). Lighter colors correspond to the excited areas.

[117, 118, 119], also called order pattern entropy, to ECG time series of ventricular fibrillation. We show that PE is able to identify periods of different complexity while using a completely different type of information than the amplitude. We observe these complexity variations in ECGs from ex-vivo experiments (Fig. 3.19 with rabbit and pig hearts. For comparison the application of PE to human ECGs from Physionet is shown (Supplemental Material in the Appendix in section B.3 on page 147.

Using numerical simulations of cardiac fibrillation in realistic geometries we show, that this phenomenon can be reproduced and appears not to be model-specific. In particular, we observe a relation between the spatio-temporal dynamics underlying the ECG and the PE-based complexity measure.

It is noteworthy that both, the fluctuations in the ECG and the complexity variations in the spatio-temporal dynamics can be interpreted from a more mathematical point of view as intermittency or intermittent phases of a nonlinear dynamical system. This perspective has also been adopted in other fields before like turbulence [120, 121], coupled map lattices 122, or coupled rings of ferrofluidic spikes [123.

\subsubsection{Methods}

\section{Animal Models and Preparation}

All animal procedures were performed in accordance with applicable local animal welfare regulations. For this study we used two New Zealand White rabbit $(3.5 \mathrm{~kg}$ and $3.0 \mathrm{~kg})$. The rabbit was injected with heparin (at least $500 \mathrm{UI} \mathrm{kg}$ ) and euthanized with sodium thiopental (at least $30 \mathrm{mg} / \mathrm{kg}$ ). Immediately after death, a high-potassium solution $(40 \mathrm{ml}$ to $100 \mathrm{ml}, 16 \mathrm{~mm}$ as described in [124]) was injected intravenously to arrest the heart and induce a plegic state. The heart was excised and kept in ice-cold cardioplegia solution until final preparation and connection to the perfusion. To suppress mechanical activity Blebbistatin was given leading to a total concentration of $2.1 \mu \mathrm{M}$ to $3.8 \mu \mathrm{M}$. In addition the 
first dataset used two cryoablation zones of $8 \mathrm{~mm}$ diameter with a small isthmus in between were created on the ventricle near the posterior branch of the coronary arteries [125] in order to facilitate the induction of VF [126] as well as flecainide (1.5 $\mu \mathrm{M})$.

\section{Heart Perfusion, Recording Setup and Induction}

Our experimental setup has been described previously [55]. In the setup, the heart is immersed in warm $\left(36.8^{\circ} \mathrm{C}\right)$ oxygenated Tyrode solution and retrogradely perfused with the same solution coming from a reservoir at $30 \mathrm{mmHg}$ to $60 \mathrm{mmHg}$.

Temperature and pressure are continuously monitored and the $\mathrm{pH}$ is periodically checked. The electrical activity on the heart is made visible using a potentiometric dye (Di-4ANEPPS, excited using cyan $(505 \mathrm{~nm})$ and green $(530 \mathrm{~nm})$ LEDs) and recorded by four EMCCD Cameras (Photometrics Evolve 128 using $610 \mathrm{~nm}$ longpass filters). In addition four measurement electrodes in the bath are used to record two ECG signals. For the second setup used for the last results section a custom-built eight lead electrode ECG was used (see the Supplemental Material). Ventricular fibrillation was induced either by local burst pacing $(50 \mathrm{~Hz})$ or by far field shocks.

\section{Numerical Simulations}

Ventricular Fibrillation was simulated, where the electrical wave propagation was modeled using the monodomain equations

$$
\begin{aligned}
\frac{\partial V_{m}}{\partial t} & =\nabla \cdot \underline{\mathbf{D}} \nabla V_{m}-I_{\text {ion }}\left(V_{m}, \mathbf{h}\right) / C_{m} \\
\frac{\partial \mathbf{h}}{\partial t} & =\mathbf{H}\left(V_{m}, \mathbf{h}\right),
\end{aligned}
$$

where $V_{m}$ is the membrane potential and $C_{m}$ is the capacitance per unit area. The first term on the right hand side of Eq. (3.10) describes the diffusive part, where $\underline{\mathbf{D}}=\underline{\sigma} /\left(\beta C_{m}\right)$ is the diffusion tensor which is proportional to the conductivity tensor $\underline{\sigma}$. $\beta$ is the area to volume ratio of the cells.

The second term consists of the contribution from the the local cell dynamics to the membrane potential and does depend on the gating variables $\mathbf{h}$ (in addition to the membrane potential $V_{m}$ itself). For the description of the behavior of the gating variables, given by Eq. (3.11), a cell model needs to be chosen. Details about the used cell model (3V-SIM, also known as Fenton-Karma model [26]) can be found in section 2.4.2 on page 48. For simulations of the Fenton-Karma model, parameter set in Tab. A.4 has been used.

\section{Permutation Entropy}

We use permutation entropy (PE) [117, 118, 119] to quantify the complexity of ECG time series. Details can be found in the Supplemental Material. 


\section{Filament Tracking}

Details about the determination of the filaments can be found in the Supplemental Material.

\section{Phase Synchronization Index}

Details about the determination of the phase synchronization can be found in the Supplemental Material.

\subsubsection{Results}

The results section is divided into four parts:

- The first part shows the phenomenon of varying complexity in the ECG during VF of a rabbit heart, its quantification by $\mathrm{PE}$ and the reproduction of the effect via numerical simulations on a realistic rabbit heart geometry.

- In the second part we show how changes in the simulated spatio-temporal dynamics (quantified by the number of filaments (NFIL) ) are visible in the (position dependent) ECGs and the respective PEs.

- We transfer the acquired knowledge of the numerical results to the experiment and show that experimental data obtained by an eight electrode setup provides PE patterns similar to the simulated results.

- The number of filaments can so far not be measured in the experimental setup. In order to strengthen our findings that the complexity of the spatio-temporal dynamics in the bulk of the tissue can be estimated by ECG time series we show that the complexity of the PE (bulk tissue) coincides with the synchronization of excitation patterns on the surface of the heart (obtained by optical mapping technique).

\section{ECG Fluctuations in Experiments and Simulations Quantified by Permu- tation Entropy}

We show that complexity fluctuations in the ECG during VF occur both in experiments and in numerical simulations. This variation of the regularity can be quantified by PE, where a low/high entropy corresponds to time periods in the ECG with a more regular/irregular variation, respectively. A regular variation in the time series promotes the existence of specific order patterns while a more irregular variation leads in general to a more uniform order pattern distribution. Figure 3.20 shows an excerpt from the ECG (a) along with the $\mathrm{PE}$ time series (b) of an episode of VF for an ex-vivo rabbit heart experiment. The time window for the computation of the entropy values is indicated by a shaded rectangle and has a length of $0.5 \mathrm{~s}$. Periods of more irregular variation often seem to coincide with a smaller amplitude in the ECG, although this observation cannot be generalized (a counterexample is given in the Supplemental Material in the Appendix in section B.3 on page 147 for the Luo-Rudy Model). 
a)
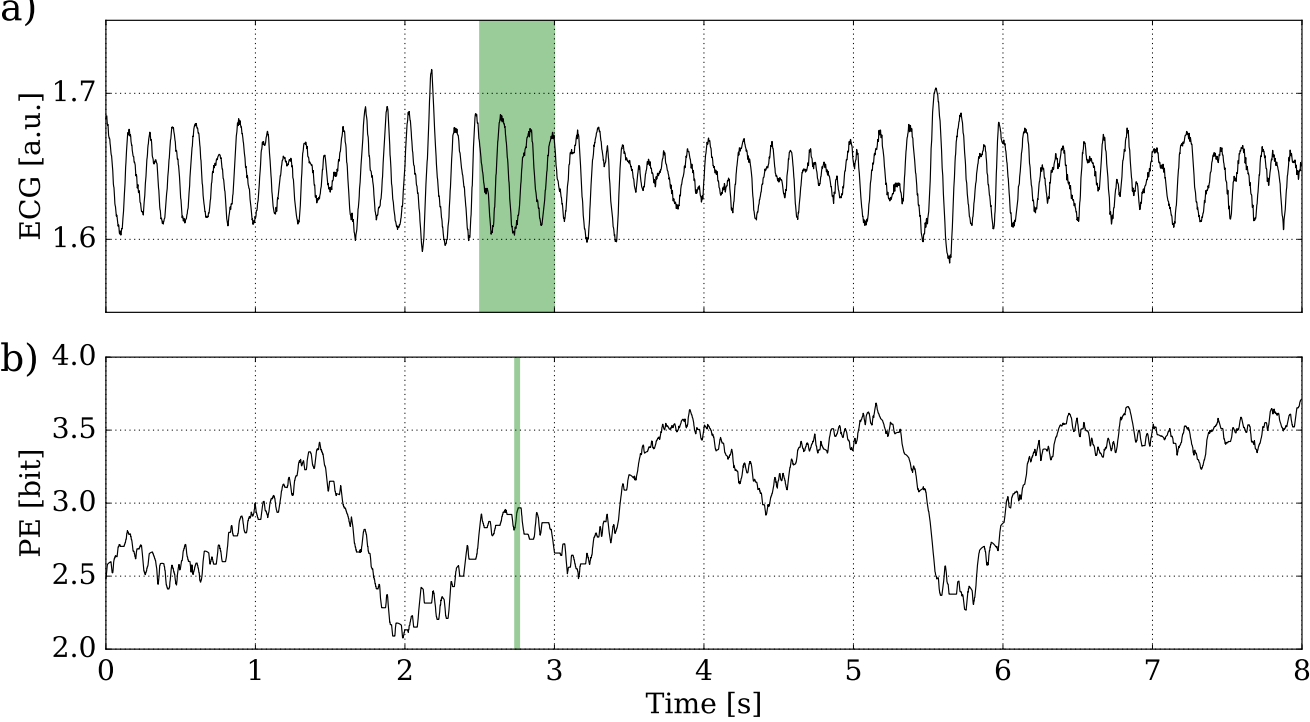

Figure 3.20: ECG (a) recorded by the attached ECG electrode (see Fig. 3.19) and PE (b) for a VF episode of a rabbit. The shaded rectangle visualizes an exemplary time window from which the corresponding PE is calculated (green line in (b)).

Further examples of complexity fluctuations during VF are shown for ECGs of human and pig (Figs. B.12 and B.13 in the Appendix in section B.3 on page 147).

From the experimental findings the question arises, whether these fluctuations are correlated with different spatio-temporal dynamics of the cardiac muscle. The idea that phases of a more regular ECG might reflect a more organized spatio-temporal excitation pattern or a better synchronization among the individual heart muscle cells had already been mentioned in first publications related to that phenomenon [115]. However, for the quantification of the dynamics of the heart, the ECG signal is not sufficient and information about the full state of the heart is desirable. Although optical mapping [55] can be used to extract the electrical excitation patterns on the surface of the heart, detailed information about the electrical wave propagation inside the tissue can so far only be obtained from numerical simulations.

In order to reproduce the observed behavior for closer investigations, we performed numerical simulations of VF (using the Fenton-Karma model [26]) on a realistic rabbit heart geometry to compare the spatio-temporal excitation patterns with the complexity of the computed pseudo ECG $_{\text {Sim }}$ [127] (calculated by Eq. (2.77)).

Figure 3.21 shows an exemplary ECG $_{\text {Sim }}$ times series (a)(exemplary observation angle of $\varphi=150$ degree, for comparison see Figs. B.7 and B.8 of a simulated episode of VF and the corresponding PE (b). Similar to the experimental results, fluctuations in the regularity of the ECG can be observed. 

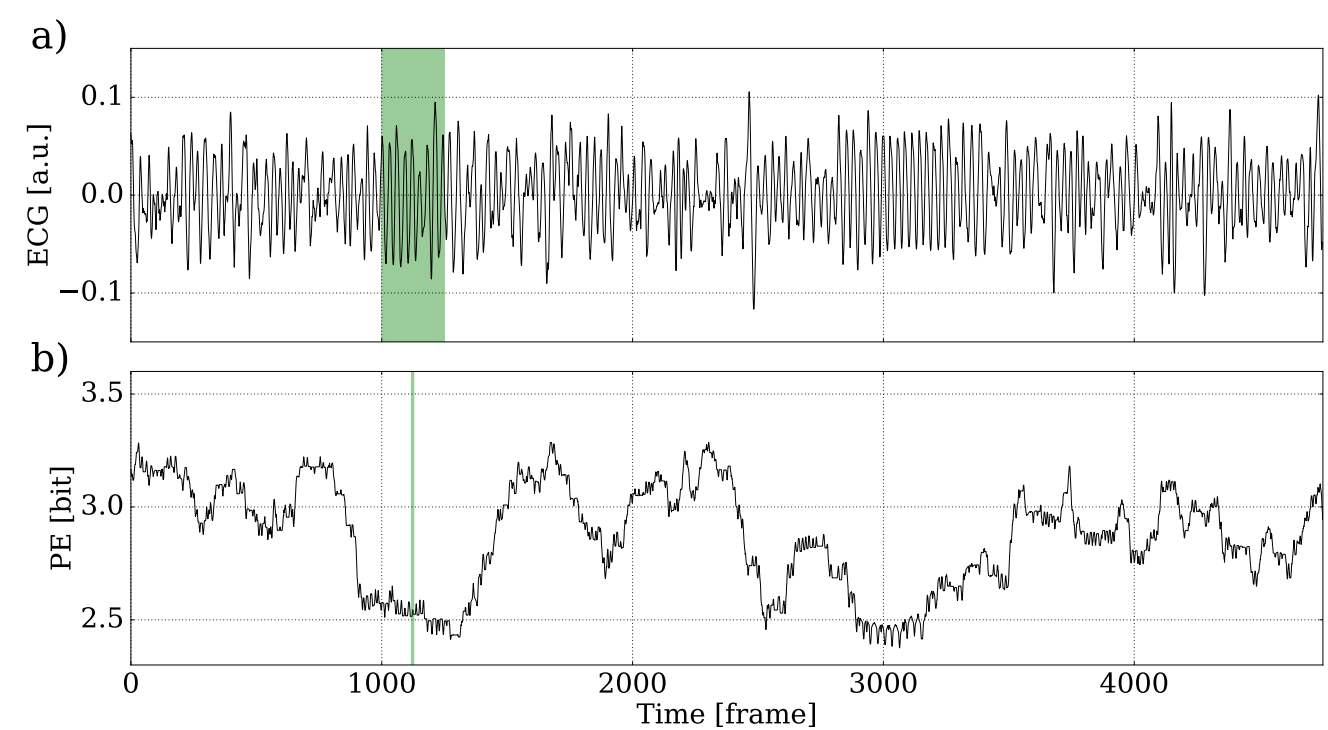

Figure 3.21: Pseudo ECG (a) computed from a simulated episode of VF using a realistic rabbit heart geometry. The ECG was determined by Eq. (2.77) (exemplary position of ECG electrode: $\varphi=150$ degree (see Figs. B.7 and B.8)). Subplot (b) depicts the corresponding PE. The shaded rectangle (a) visualizes an exemplary time window from which the corresponding PE is calculated (green line in (b)).

a)

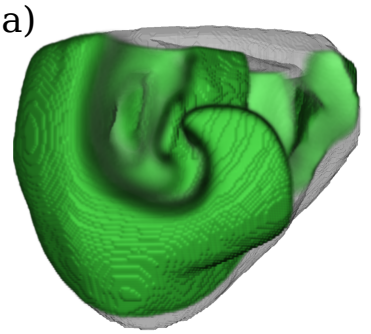

b)

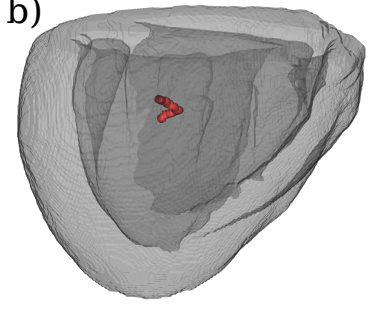

c)

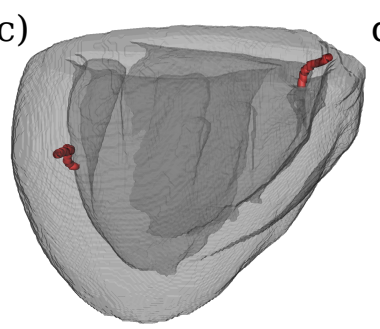

d)

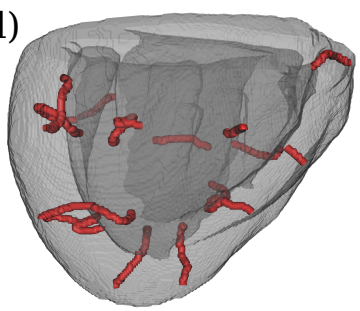

Figure 3.22: Example of how the dynamics of a scroll wave (a) is reduced to the corresponding filament (b). Subplots (c) and (d) present examples of a laminar state (time step 1085 in Fig. 3.23) and a complex state (time step 2140 in Fig. 3.23), respectively.

\section{Correlations between the Spatio-Temporal Dynamics and Angle Depen- dent ECGs}

Exploiting the knowledge about the full state of the system in numerical simulations, the spatio-temporal origin of the discussed fluctuations can now be further investigated. In order to quantify the spatio-temporal complexity of the system, we determined the number of filaments (NFIL) during the episodes of simulated VF, which is directly related to the number of scroll waves inside the heart (Fig. 3.22).

In addition, the ECG was calculated for various ECG electrode positions. For this purpose, we used the infinite volume conductor approach, which provides a position sensitive and dipole source based ECG $_{\text {Sim }}$ (Eq. 2.77). We positioned the ECG electrode midventricular around $4 \mathrm{~cm}$ away from the epicardium, and rotated under the angle $\varphi$ around the heart 


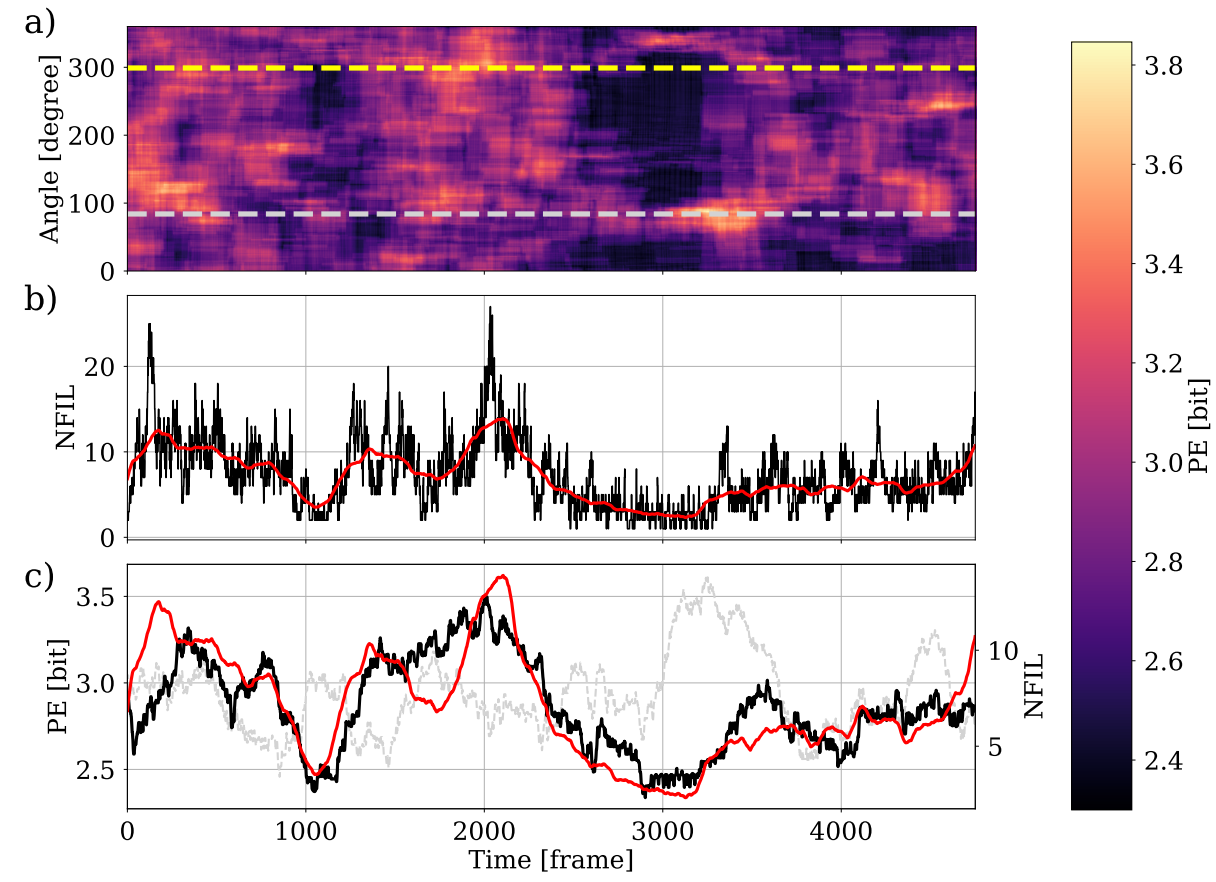

Figure 3.23: This figure compares the Permutation Entropy as a complexity measure of the position dependent ECG signals with the number of filaments (NFIL) as a measure of the complexity of the spatio-temporal state during an episode of simulated VF. Each point in (a) is the color-coded value of the PE (calculated based on the position sensitive ECG $_{\text {Sim }}$ (Eq. (2.77) )) at a specific point in time and corresponds to the ECG signal measured under a specific angle. Subplot (b) shows NFIL over time (the raw number is black and a smoothed number is plotted in red). The horizontal yellow (gray) dashed line in (a) at 299 degrees (84 degrees) depicts the angle, where the corresponding $\mathrm{PE}$ exhibits the maximum (minimum) correlation of $C=0.81(C=-0.33)$ for the whole time series. In order to illustrate this correlation, the actual PE is additionally plotted in subplot (c) as the black (light gray) curve, together with the smoothed version of NFIL (red line).

(Figs. B.7 and B.8 in section B.3 on page 147).

In Fig. 3.23 we present the analysis of an episode of simulated VF with a distinct phase of low complexity (low number of NFIL): In subplot (a) the PE of the pseudo $\mathrm{ECG}_{\text {Sim }}$ recorded under the observation angle $\varphi$ is sketched color coded, whereas the number of filaments NFIL is shown in (b) (in black, smoothed curve in red). The number of filaments, and thus scroll waves, is fluctuating during the episode, and reaches low values e.g. at around time step 1100 or time step 3000, indicating a phase of low spatio-temporal complexity. Snapshots of the spatio-temporal dynamic state of the heart characterized by the filaments are given in Fig. 3.22 for two exemplary states: a "laminar state" (corresponding to a low NFIL and thus a low level of complexity) is shown in (c), taken at time step 1085 and a "complex state" (corresponding to a high NFIL and thus a high level of complexity), taken at time step $2140(\mathrm{~d})$.

These fluctuations are also visible in the PE spectrum, where the actual correlation depends on the observation angle of the corresponding ECG. The yellow (gray) horizontal line 


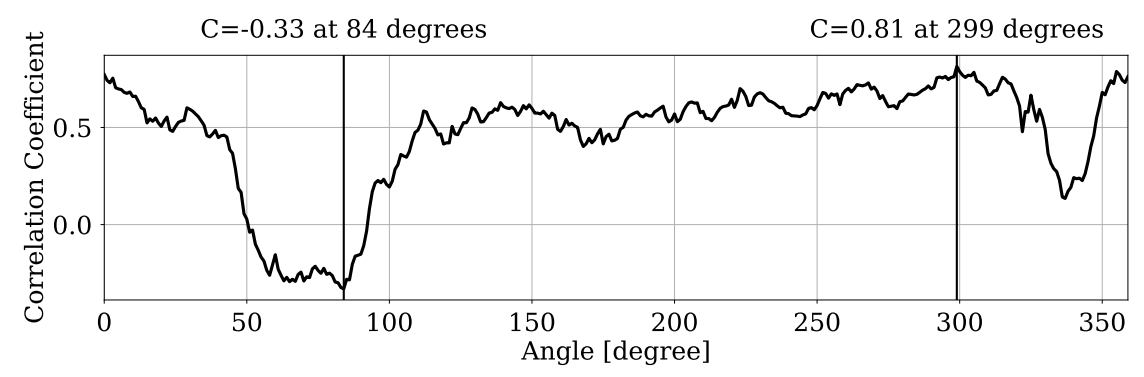

Figure 3.24: The dependency between the angle of the ECG electrode and the correlation coefficient calculated from NFIL and PE (for the episode of VF discussed in this section). Two vertical black lines indicate the highest (lowest) correlation at an angle of 299 degree (84 degree). Their corresponding PEs are plotted explicitly in Fig. 3.23(c) as the black (gray) line, respectively. The correlation coefficient for each angle is calculated on the basis of the whole time series (5000 frames).

in Fig. 3.23(a) indicates the PE with the highest (lowest) correlation with NFIL. Both PE are additionally plotted together with the smoothed NFIL in (c) as the black (highest correlation, $C=0.81$ ) and light gray (lowest correlation, $C=-0.33$ ) curves. The correlation coefficient between the PE and NFIL is also shown in Fig. 3.24 for each observation angle of the corresponding ECG. The reason for the varying correlation coefficient is the limited "range" of a single ECG electrode: primarily the excitation patterns in the proximity of an ECG electrode contribute to the signal measured at a specific position (thus, at a particular observation angle) $\left(\sim \frac{1}{r^{2}}\right.$ in Eq. (2.77) $)$. That means, the time series of a single ECG electrode provides information only about a (nearby) section of the whole heart. The "laminar" phase around time step 3000 reflects this mechanism: Most ECG electrodes do not observe a complex dynamics (low PE in Fig. 3.23(a)). However, the (low number of) scroll waves which are still present in the system cause a high $\mathrm{PE}$ in a small range of observation angles (around $\varphi \sim 80$ degree) which are close to the organizing center of the remaining scroll wave.

From the above analysis we draw two conclusions: A single (spatially fixed) ECG electrode is not enough to evaluate the spatio-temporal state of the system, since it only measures a projected signal (under the respective observation angle) from the overall excitation pattern. Furthermore, a perfect correlation is not possible, even when all electrodes are taken into account, since e.g. in a laminar phase, some of them will detect the remaining scroll waves of the system and therefore provide a high PE. Regarding potential applications, it is of great interest which number and geometry of electrodes is appropriate in order to make a reliable statement about the spatio-temporal complexity of the heart. For this purpose we chose different numbers of equally spaced electrodes and calculated the average correlation between NFIL and the PE concerning all electrodes used. Figure 3.25 shows the average correlation coefficient and standard deviation for different numbers of equally spaced electrodes (for a fixed number of electrodes averaged in one degree steps over all possible arrangements) for the initial condition discussed in this section. The average correlation saturates at approximately $C_{\mathrm{Sat}} \approx 0.78$, and already the use of five equally spaced electrodes provides a 


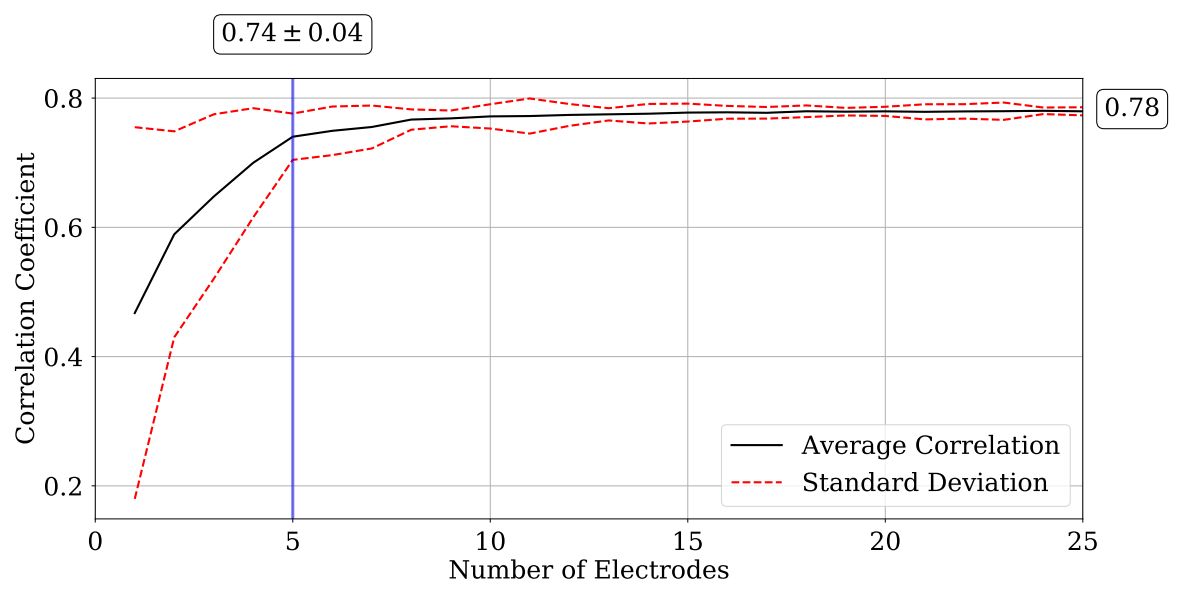

Figure 3.25: This figure shows the correlation between NFIL and the mean of PEs (concerning selected electrodes) for different numbers of equally spaced electrodes for the initial condition discussed in this section.

mean correlation of $C_{5} \approx 0.74 \pm 0.04$. In total eighteen episodes of VF were simulated (each episode containing of 5000 time steps). The saturated correlation and the value for five electrodes for all eighteen initial conditions investigated in this study are shown in Tab. 3.3 . The average over all initial conditions is $C_{\text {Sat }}^{\mathrm{Mean}} \approx 0.64 \pm 0.15$ and $C_{5}^{\mathrm{Mean}} \approx 0.59 \pm 0.15$.

This analysis indicates that a relatively low number of electrodes can be sufficient to make a reliable statement about the spatio-temporal complexity of the heart based on ECG time series.

\section{Experimental Results Using an Eight-Electrode Setup}

We showed in the previous section that in numerical simulations one can (to some degree) estimate the complexity of the spatio-temporal dynamics of the heart using a finite amount of ECG electrodes. Now we want to verify these findings in an experimental setup. For this purpose, an eight-electrode setup was used in an ex-vivo rabbit heart experiment in order to measure position dependent ECG signals during episodes of VF. A sketch of the setup can be found in the Supplemental Material (Fig. B.10).

Figure 3.26 presents the analysis of an episode of VF of about $10 \mathrm{~s}$ in an ex-vivo rabbit heart experiment. Subplot (a) shows the color coded PE (based on the eight ECG electrodes). In the time window between $2 \mathrm{~s}$ and $4 \mathrm{~s}$ a similar structure as in Fig. 3.23 (a) can be observed (only one ECG electrode with a high PE), indicating a "laminar" phase, with a low number of scroll waves.

\section{Estimation of Surface Synchronization from PE}

The PE data obtained from the ECGs of the eight-electrode setup yielded similar patterns than the ones we found in numerical simulations. In the experimental setup, however, we 
Table 3.3: This table shows the saturated correlation $C_{\text {Sat }}$ between NFIL and PE using different numbers of equally spaced electrodes for 18 initial conditions in addition to the average correlation using five electrodes $C_{5}$ (averaged over all possible arrangements of the respective electrodes). The initial condition concerning Simulation ID 4 is discussed in the results section.

\begin{tabular}{|c|c|c|c|c|c|}
\hline Simulation ID & $C_{5}$ & $C_{\text {Sat }}$ & Simulation ID & $C_{5}$ & $C_{\text {Sat }}$ \\
\hline 1 & 0.78 & 0.83 & 10 & 0.48 & 0.54 \\
\hline 2 & 0.65 & 0.71 & 11 & 0.68 & 0.72 \\
\hline 3 & 0.61 & 0.66 & 12 & 0.48 & 0.54 \\
\hline 4 (Fig. 3.23 ) & 0.74 & 0.78 & 13 & 0.78 & 0.84 \\
\hline 5 & 0.27 & 0.33 & 14 & 0.74 & 0.79 \\
\hline 6 & 0.54 & 0.59 & 15 & 0.61 & 0.66 \\
\hline 7 & 0.70 & 0.75 & 16 & 0.39 & 0.45 \\
\hline 8 & 0.64 & 0.70 & 17 & 0.74 & 0.81 \\
\hline 9 & 0.34 & 0.37 & 18 & 0.45 & 0.51 \\
\hline
\end{tabular}

can so far not detect the number of scroll waves or the related number of filaments as we did in the simulation study. In order to strengthen the observed relation between the spatiotemporal dynamics and the $\mathrm{PE}$ in the experimental case, we compare in this section the $\mathrm{PE}$ based on the eight-electrode setup with the electrical excitation patterns on the surface of the heart, using the optical mapping approach. In detail, we compare the fluctuations of the PE with the level of synchronization of the electrical excitation patterns, calculated by the Phase Synchronization Index (PSI] for details see the Supplemental Material). Essential for this analysis is that in general the dynamics observed on the surface (measured by optical mapping) contains only limited information about the full 3D dynamics of the bulk tissue. In particular, a high level of complexity of the spatio-temporal dynamics of the bulk does not necessarily generate complex excitation patterns on the surface. However, in the other direction, an organized spatio-temporal state will also cause a high level of synchronization on the surface.

We verify this assumption by calculating the error of a nearest neighbor prediction (similar to the approach described in [128]) that predicts the value of PSI using PE information obtained from the eight electrodes of the ECG. Let $y\left(t_{j}\right)=\left\{\mathrm{PE}_{1}\left(t_{j}\right), \ldots, \mathrm{PE}_{8}\left(t_{j}\right)\right\}$ be the vector of $\mathrm{PE}$ from eight electrodes at discrete times $t_{j}(j \in\{1, \ldots, N\})$. By randomly selecting half of the possible $j$ we generate a training set $Y_{a}=\left\{y\left(t_{k_{1}}\right), \ldots, y\left(t_{k_{N / 2}}\right)\right\}$ with associated values of PSI $P_{a}=\left\{\operatorname{PSI}\left(t_{k_{1}}\right), \ldots, \operatorname{PSI}\left(t_{k_{N / 2}}\right)\right\}$. The remaining values form the test sets $Y_{b}$ and $P_{b}$ accordingly. For each element of $Y_{b}$ with $t=t_{k_{m}}, m \in\{N / 2+1, \ldots, N\}$ the nearest neighbor in $Y_{a}$ with $t=t_{l\left(k_{m}\right)}$ is found. The prediction error for PSI can then be estimated by computing the RMSD (root mean squared deviation) as follows:

$$
\operatorname{RMSD}=\sqrt{\frac{2}{N} \sum_{m=N / 2+1}^{N}\left(\operatorname{PSI}\left(t_{l\left(k_{m}\right)}\right)-\operatorname{PSI}\left(t_{k_{m}}\right)\right)^{2}}
$$


a)

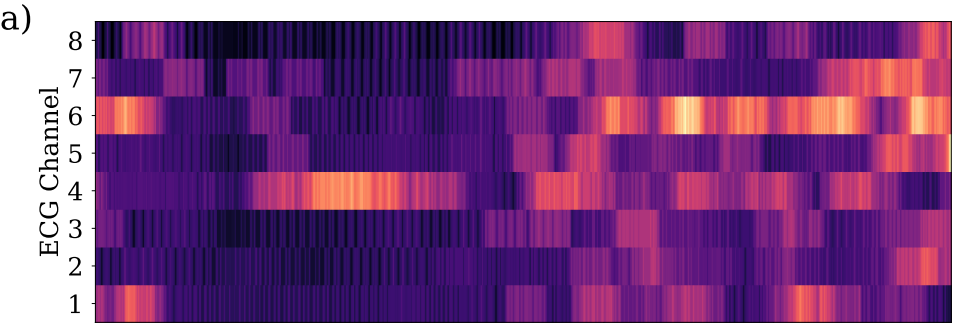

b) 0.7
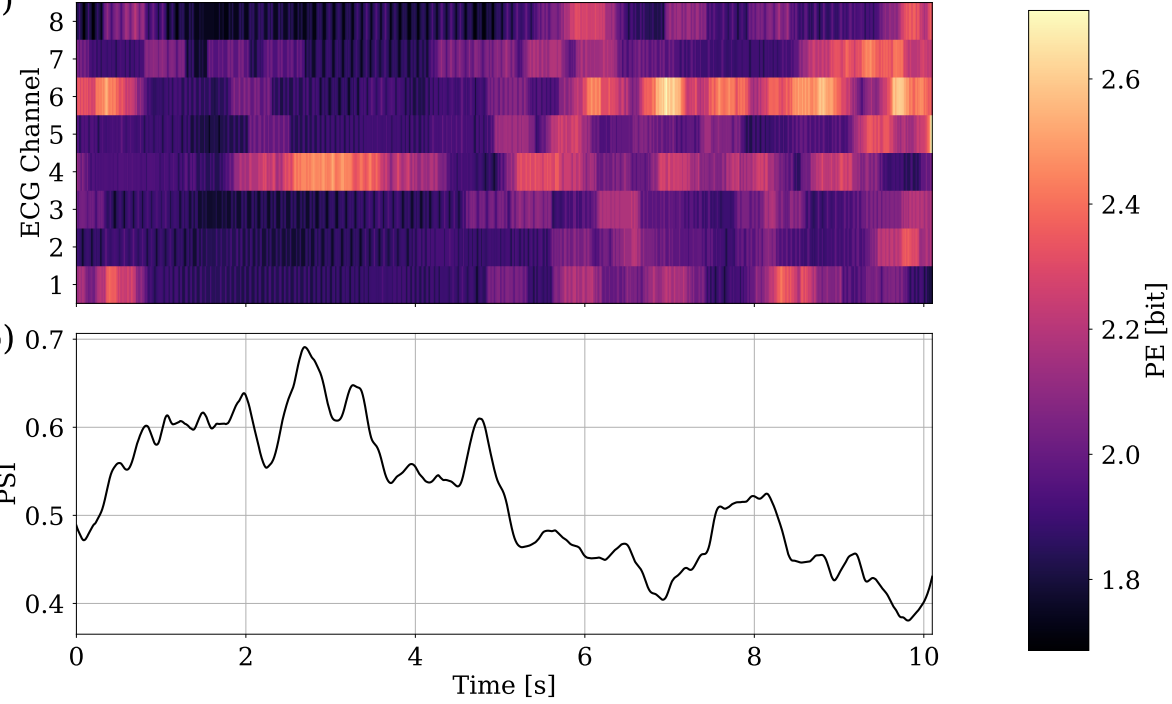

Figure 3.26: Analysis of complexity fluctuations during an episode of VF in a rabbit heart using an eight-electrode setup. Subplot (a) shows the (color coded) PE calculated based on the eight ECG electrodes used in the experimental setup. Between $2 \mathrm{~s}$ and $4 \mathrm{~s}$ the PE of all ECG electrodes is low except one (ECG channel 4), indicating a laminar phase. For comparison, the PSI (based on data obtained from optical mapping) is shown in (b). During the phase of only one high PE, also the PSI indicates a relatively high synchronization on the surface of the heart.

In order to estimate the significance of the RMSD obtained with Eq. (3.12) a surrogate procedure is used: 1000 surrogates are generated by shuffling the order of the PSI values and therefore destroying the association between the PE vectors $y$ and the PSI values. The distribution of RMSD for the surrogates can then be computed with Eq. (3.12). To avoid unwanted correlations between the data points a subsampling of two points per second has been used.

We applied this procedure to a dataset consisting of $34 \mathrm{VF}$ episodes from a single rabbit heart with a total duration of $917 \mathrm{~s}$. The result is shown in Fig. 3.27. The blue line on the left marks the RMSD with the correct association of PE vectors $y$ and PSI. The distribution of RMSD for the surrogates can be seen on the right. As the real RMSD and the distribution of RMSD for the surrogates are clearly separated we can assume that the association between PE and PSI contains additional information which can be used to predict PSI from PE obtained from eight-electrode ECG measurements. This means that the ECG measurements contain and provide relevant information about the complexity of the underlying spatio-temporal process.

\subsubsection{Conclusion}

We have shown that permutation entropy can be used to identify phases of different complexity in the ECG during VF of several species. Using numerical simulations we were able to establish a link between these fluctuations and changes in the spatio-temporal dynamics, 


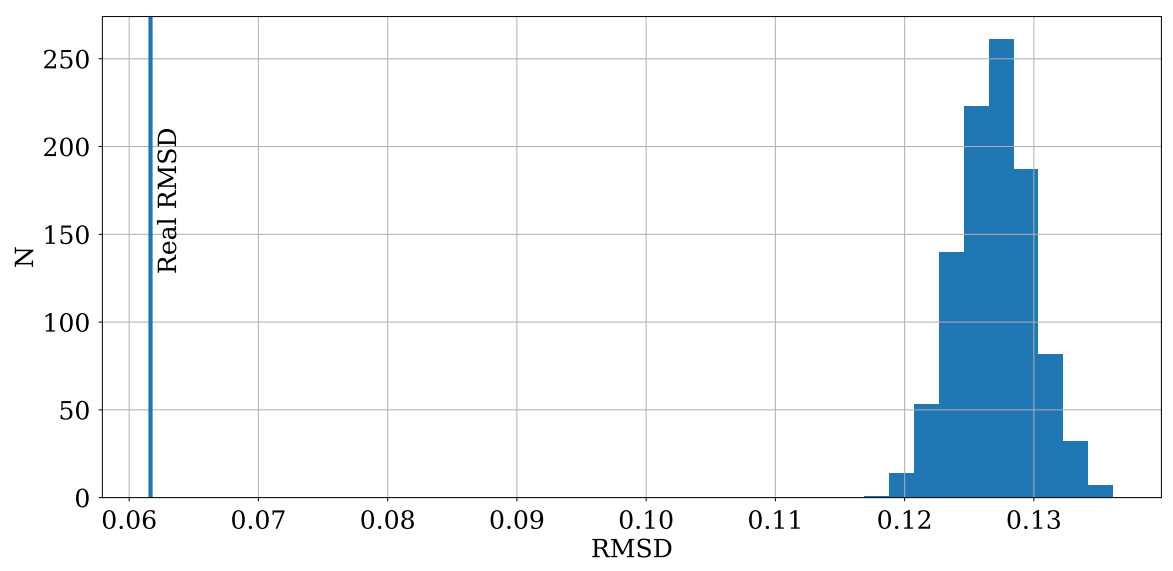

Figure 3.27: Comparison of the Real RMSD (defined by Eq. (3.12) with the RMSD distribution based on 1000 surrogates. The clear separation of the real value and the surrogates indicates a robust correlation of the PE calculated by the eight-electrode ECG setup, and the spatio-temporal synchronization of excitation patterns on the surface of the heart.

which we quantified by the number of filaments. We found that the correlation between the number of filaments and the PE depends on the position of the corresponding ECG electrode and thus in a laminar phase, some ECG electrodes still indicate a high level of complexity since due to their spatial proximity they detect the dynamics of the remaining scroll waves (whose number is, however, globally low). Taking this effect into account, we conclude that a perfect correlation is therefore not possible. However, five equidistant electrodes can on the one hand in average provide a reliable statement about the global level complexity of the dynamics inside the heart in simulations. Yet, in laminar phases, they even provide more detailed information about the localization of the remaining scroll wave(s).

Using an eight-electrode setup in ex-vivo experiments of a rabbit heart, we found similar patterns of the permutation entropy as in the simulation study. The comparison of the PE and the level of synchronization (determined by the Phase Synchronization Index) of the excitation patterns on the surface of the heart (obtained by the optical mapping technique) supports our hypothesis that a statement about the level of complexity during episodes of VF can be obtained by a many-electrode setup also in the experimental case. Being able to identify phases of different complexity in the ECG can have implications for the classification of cardiac arrhythmias. Furthermore, this knowledge may be exploited for improvements of defibrillation techniques [21], concerning a complexity dependent defibrillation technique [129]. 


\section{Summary of Results}

In this study, we could verify a fluctuating number of filaments, which are used here as a measure for the level of spatio-temporal complexity of the dynamics (Fig. 3.28). Actually, the fluctuating number of filaments represents the ongoing creation and annihilation of scroll waves. That means, these fluctuations represent also the underlying mechanism for the process of self-termination, studied in the previous studies.

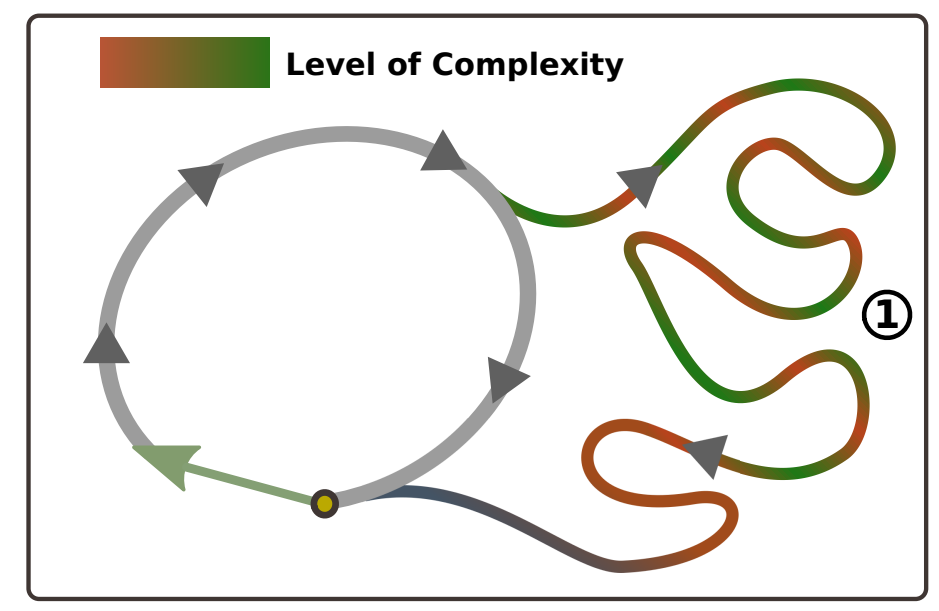

Figure 3.28: The (schematic) state space, depicting the conclusion of the study "Unveiling Spatio-Temporal Complexity Fluctuations in Cardiac Arrhythmia Using Permutation Entropy of ECG Time Series". The main result is sketched using an exemplary trajectory, which represents a typical chaotic episode of ventricular fibrillation (1). Different levels of spatio-temporal complexity are illustrated here color coded (transition between red and green).

Furthermore, the fluctuating number of scroll waves is measurable also in ECG time series. The level of complexity of multiple ECG time series, determined by the calculation of Permutation Entropy (by Alexander Schlemmer), correlates with the number of filaments. We showed furthermore, that the number and position of the ECG electrodes is essential for a reliable estimate of the spatio-temporal level of complexity. These findings were then also verified in the experimental setup (performed by Tariq Baig, Sebastian Berg, Rabea Hinkel, Daniel Horning and Claudia Richter) using a multiple ECG electrode setup, built by Laura Diaz Rodriguez.

The discussion of the results and the implications for possible applications can be found in the section "Discussion and Outlook" 4.1 on page 125. 


\subsection{Termination via Perturbation}

In the previous studies, the state space of spatio-temporal chaos was investigated and characterized. After these rather descriptive analyses, we now want to exploit the obtained knowledge and actively interact with the system. In particular, similar to the study of section 3.2 on page 76 perturbations are used to modify trajectories.

\section{Scientific Objective}

The governing question of this study is directly related to the main motivation of this thesis how the side-effects of the conventional defibrillation technique can be reduced. The objective is how the chaotic spatio-temporal dynamics in excitable media can be efficiently terminated with the lowest strength of interaction possible. The strength of interaction is in this sense evaluated by the resulting side-effects of the interaction with the system. Concerning the application of a far field shock, reducing the electrical field strength of the shock reduces the induced currents, and in this way the side-effects. But, using a low field strength results also in a decreasing number of virtual electrodes (see section 2.1.5 on page 22, and a lower success rate (see for example the dose response curve in Fig. 2.10.

In the previous study of section 3.2 on page 76 the structure of the state space was investigated. In particular, related to the transient nature of the dynamics, the fractal properties have been revealed, concerning the transient lifetime of perturbed trajectories (e.g. Fig. 5 (b) in the study of section 3.2 on page 76 . This observation can be interpreted in the following way: Assume a typical trajectory at a point $\mathbf{x}$ in state space, with a specific transient lifetime of $T_{1}$ (measured from $\mathbf{x}$ ). Then, there exist in the neighborhood of $\mathbf{x}$ a close trajectory $\mathrm{x}^{*}$, which can be reached by small perturbations, and which has a transient lifetime $T_{2}$ which is significantly shorter than $T_{1}{ }^{2}$

From these consideration, the scientific objective of this study can be formulated: The task is, to find perturbations as small as possible, applied to trajectories, leading to perturbed trajectories with transient lifetimes which are as short as possible. Subsequently, a transient lifetime which is short enough (what "short enough" means needs to be adjusted and refers to relevant time scales of the applied system) can be considered then as termination. This strategy can also be illustrated in the state space (Fig. 3.29), where the mechanisms of the conventional defibrillation (blue arrow) and the (low impact) perturbation approach are significantly different.

The process of recruiting virtual electrodes is essential for the understanding of the main mechanisms which play a role during a defibrillation event. When considering the recruitment of virtual electrodes as perturbing the system, space of possible perturbations is very limited due to the spatial distribution of the virtual electrodes. In fact, inducing, for example, excitation only at a specific spot of the tissue is in most cases not possible via the application of an external electrical field. That means, the presented method where the system is perturbed (excited) at specific positions is not directly applicable in practice.

\footnotetext{
${ }^{2}$ Actually, there exists also nearby states with a lifetime larger than $T_{1}$. However, since we are interested in terminating the dynamics, a short lifetime is desired.
} 


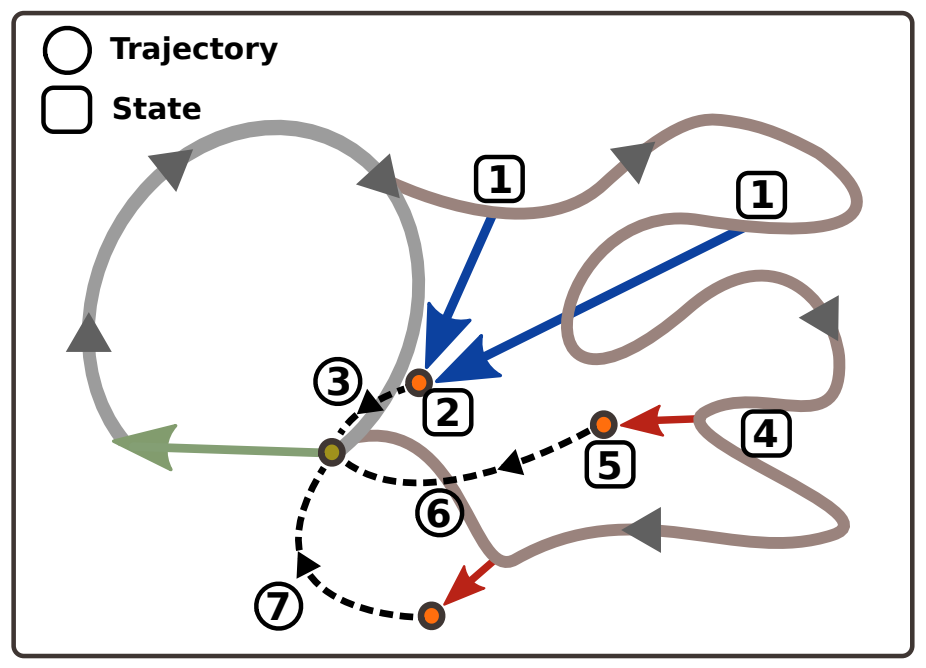

Figure 3.29: The (schematic) state space, formulating the scientific objective concerning the study "Termination Via Perturbation". The concept of conventional defibrillation, interpreted as a perturbation of the trajectory in the state space is depicted as blue arrows. The defibrillation shock is applied at specific points in time (two exemplary states are denoted by (1)). The strength of the electrical field recruits virtual electrodes on all scales, and the shock excites the whole tissue. That means, the conventional defibrillation method perturbs the system always to a specific state (2), where the whole tissue is excited at the same time. Then, the whole tissue repolarizes at the same time, thus going back to the resting state (3). In contrast to the conventional defibrillation, a possible low-energy approach interacts less with the system (red arrows). This technique perturbs the state to a nearby state (5). From here, the system also evolves to the resting state. Since these nearby states are state specific, the system returns back to the resting state on different paths ((6) and (7)). This is a central feature which is in contrast to the case of conventional defibrillation, where the defibrillation shock brings the system always in the unique state of global excitation (2), before it returns to the resting state (3).

However, the goal of this study is to terminate the chaotic dynamics in numerical simulations in the most efficient way, as a proof of principle. In a second step, the results need to be analyzed, general laws, dependencies, and mechanisms have to be identified, which can then influence and motivate the development of novel defibrillation concepts.

\subsubsection{General Concept}

Due to the high-dimensionality of the investigated systems, there are extremely many possible perturbations which can be applied to trajectories. S. P. Cornelius et al. developed a general algorithm, how in numerical simulations of high-dimensional networks a desired state can be achieved by the application of a final perturbation which is composed of many small perturbations [130]. For the purpose of clarity, we denote from now on these small constituents just as perturbations, whereas the final collective "push", composed of the small perturbations as the directed shift. For the application, the knowledge of the underlying equations or mapping rules of the dynamics are required. This algorithm is used in this study, to identify directed shifts which lead to a fast termination of the perturbed trajec- 


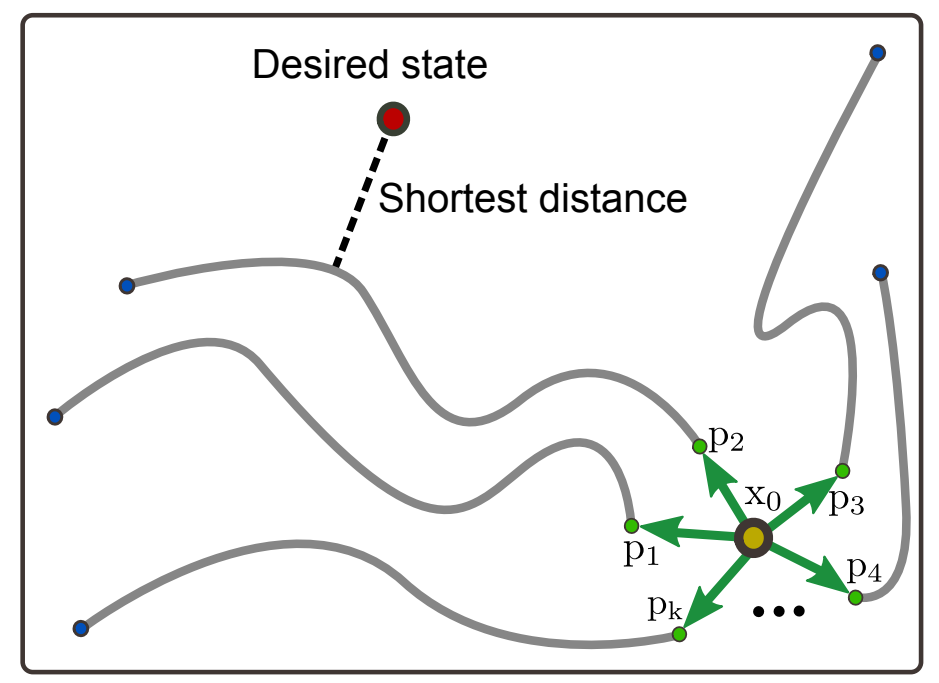

Figure 3.30: The principle of the network control problem, shown for a specific example of spatio-temporal chaos in excitable media. Perturbations $\mathrm{p}_{1} \ldots \mathrm{p}_{k}$ are applied to the point in state space $\mathbf{x}_{0}$, leading to $k$ new perturbed trajectories. Subsequently, the perturbation which corresponds to the trajectory which comes closest to the desired state during $\mathrm{T}_{\mathrm{Dev}}$ is chosen for the next iteration of the algorithm.

tory (preferably short transient time). In the following, we give a broad outline of the algorithm:

1. Choose a specific trajectory, and a specific point in time ( $\mathbf{x}_{0}$ in Fig. 3.30 when the directed shift should be applied (thus a specific point in the state space).

2. Apply all possible perturbations one by one (denoted by $\mathrm{p}_{1} \ldots \mathrm{p}_{k}$ ), and let the system evolve for a certain amount of time $\mathrm{T}_{\mathrm{Dev}}$ (which is a parameter of this algorithm). Only one perturbation at once is applied, thus with $k$ possible perturbations, this procedure results in $k$ new perturbed trajectories.

3. If the trajectory which corresponds to a specific perturbation reaches the desired state during the time interval, the algorithm is finished. If not, one needs to determine which of the perturbed trajectories comes closest to the desired state during $\mathrm{T}_{\mathrm{Dev}}$. A metric has to be chosen here, which determines the distance between different states in state space.

4. The perturbation which corresponds to the trajectory which comes closest to the desired state ( $\mathrm{p}_{2}$ in Fig. 3.30) will be kept, all other perturbations are ignored.

5. The old state $\mathbf{x}_{0}$ together with the chosen perturbation $\mathrm{p}_{2}$ is the new starting point in state space. That means, all kinds of perturbations are applied again at this new point $(\rightarrow 2$.). The algorithm goes on, until so many perturbations are collected, that the sum of them perturbs the original trajectory in such a way, that the new trajectory reaches the desired state. 


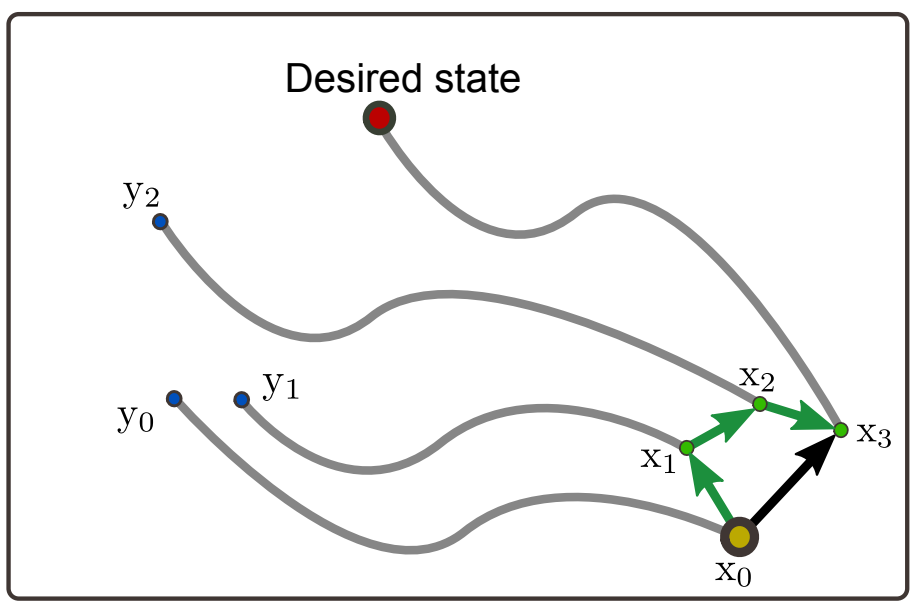

Figure 3.31: The principle of the network control problem, shown for a specific example of spatio-temporal chaos in excitable media. At the end of the algorithm, the directed shift (black arrow) which is composed of small perturbations shifts the original state $\left(\mathbf{x}_{0}\right)$ to a nearby state $\left(\mathbf{x}_{3}\right)$ which will end up in the desired state during the evolution time $\mathrm{T}_{\text {Dev }}$.

In this way, the algorithm creates a final perturbation vector (directed shift), which is composed by many small perturbations. These perturbations shift the original trajectory in such a way, that it reaches the desired point in state space during $\mathrm{T}_{\text {Dev }}$ (Fig. 3.31).

- In order to understand the procedure of the algorithm it noteworthy to mention that the purpose of performing many simulations of perturbed trajectories is, to find the proper combination of perturbations. Although during the algorithm proper perturbations add up iteratively, at the end all collected perturbations are applied simultaneously.

This algorithm is a powerful tool to determine a proper combination of single perturbations (out of the extremely high-dimensional space of possible composed perturbations), which terminates the dynamics within $\mathrm{T}_{\text {Dev }}$. However, this method follows a greedy strategy and does not provide the certainty that a particular combination of perturbations is the best possible choice in terms of the lowest number of perturbations. Still, the algorithm provides the useful possibility to "navigate" systematically in the high-dimensional space of possible shifts.

\subsubsection{Implementation of the Algorithm}

Since this study relates to the previous study of section 3.2 on page 76 , the system we investigate here is a two-dimensional simulation of excitable media $\left(\mathrm{L}_{\mathrm{x}} \times \mathrm{L}_{\mathrm{y}}=100 \times 100\right)$ using the Fenton-Karma model for the local cell dynamics (parameter set FK1 (Tab. A.2 on page 132, with $\mathrm{D}=0.2, h=1.0, d t=0.02)$. 206 episodes of spatio-temporal chaos were created (with the same protocol as used in section 3.2) which exhibit chaotic dynamics for at least 100 spiral periods. 
Perturbations were modeled as a local external electrical current. That means, any perturbation locally increased the membrane potential. The current was applied at circular areas with a radius of $r_{\text {Pert }}=1$, corresponding to five pixels of the simulation grid, during one arbitrary time unit (equal to 50 simulation step). The strength of the perturbation is equivalent to a change of the membrane potential $V_{m}$ of approximately $300 \mathrm{mV}$. Due to the strong influence of diffusion in the simulations, this did not lead to significantly unphysical values of the membrane potential $V_{m}$.

The set of possible perturbations is constrained to the membrane potential. Furthermore, possible positions of the perturbations were fixed to a grid of $50 \times 50$ points, also illustrated in Fig. 3(a) of the study of section 3.2 on page 76 . That means, in total 2500 different perturbations can be applied. The distance between two states in the state space is determined by the Euclidean metric, applied to the state vectors of the membrane potential $V_{m}$. The desired state for all simulations is the resting state, that means the absence of any excitation patterns. Furthermore, after each iteration of the algorithm, the number of phase singularities, and consequently the number of spiral waves was determined. When no spiral wave was left in the system, the algorithm was successful and finished.

An example of an application is shown in Fig. 3.32 , where a specific state $\mathrm{x}_{0}$ (membrane potential $V_{m}$ is shown in (a)) is considered. Phase singularities are determined and depicted as white dots. The evolution time is in this example $\mathrm{T}_{\mathrm{Dev}}=500$ time units, which corresponds to approximately 5 spiral rotations. Without any perturbation, the system evolves to $y_{0}(b)$. After the first iteration of the algorithm, the best perturbation has been determined (marked by the green circle (c)). The perturbed trajectory evolves and exhibits a less chaotic dynamics (d) than without the perturbation (b). The second perturbation (e) further reduces the number of spiral waves at the end of the evolution time (f) and by adding the third perturbation ( $\mathrm{g}$ ) no phase singularity is left $(\mathrm{h})$. Note that for the purpose of visibility, green circles which illustrate the perturbations are larger than the actual area of perturbed cells.

In the selected example shown in Fig. 3.32 a remarkable phenomenon can be observed, which occurred in many of the investigated cases: When comparing the final states of the system $y_{1}, y_{2}$, and the "Desired State", excitation patterns are not completely different, but it seems that the newly added perturbations act only on a spatially constrained part of the domain. Specifically, new perturbations terminate spiral waves in specific regions, but have no impact on other locations. This gives rise to the hypothesis, that the whole domain can be divided into smaller parts or "tiles" as proposed by Byrne et al. [131] and for a successful termination of the dynamics each part needs to be controlled independently.

\subsubsection{Results}

In total, 206 independent trajectories were investigated and the parameter $T_{D e v}$ was varied between 100 to 1000 time units, which is equivalent to approximately 1 to 10 spiral periods. A maximal amount of 15 perturbations were applied. In a proof of principle, we demonstrated that the chaotic dynamics in the investigated high-dimensional system 
(a)

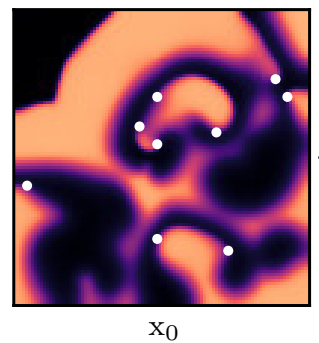

(c)

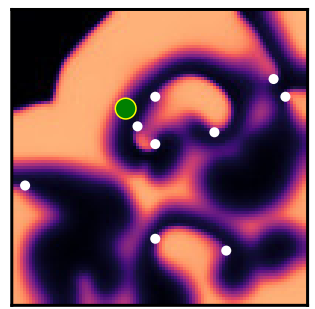

$\mathrm{x}_{1}$

(e)

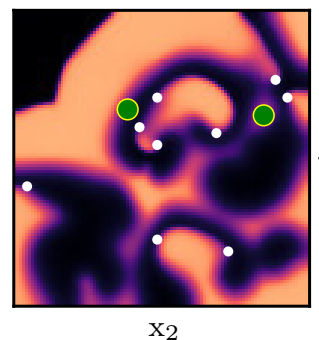

$\mathrm{x}_{2}$

(g)

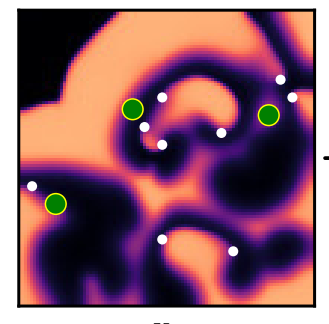

$\mathrm{x}_{3}$ (b)

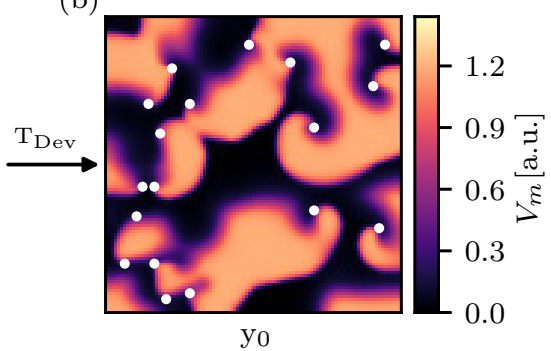

(d)

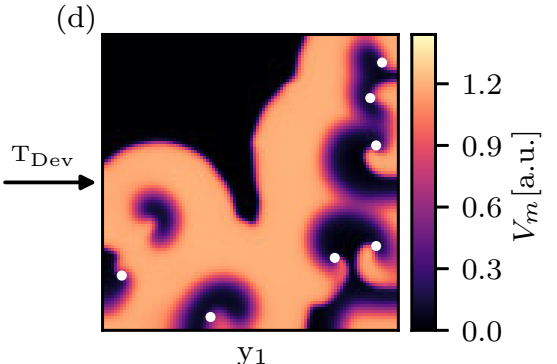

(f)
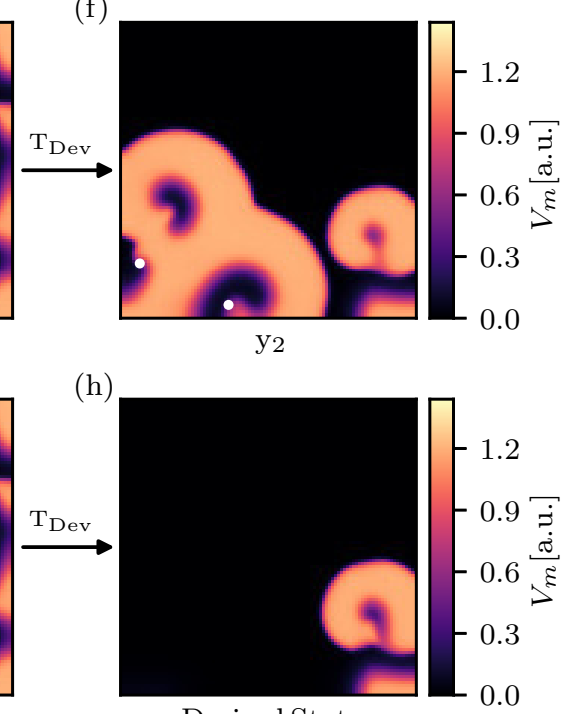

Desired State

Figure 3.32: Selected snapshots of a specific example of spatio-temporal chaos, illustrating the principle of the network control algorithm. The membrane potential $V_{m}$ is shown, whereas white circles indicate phase singularities and green circles depict the positions of the applied perturbations. In (a), the state $\mathrm{x}_{0}$ is shown where the perturbations are applied. Subplot (b) depicts the evolved state (after $\mathrm{T}_{\mathrm{Dev}}$ ). The first perturbation which is determined by the algorithm is depicted in (c) as the green circle and the evolution of the perturbed state in (d). Adding another perturbation to the initial state (e), reduces the number of spiral waves significantly (f), and three perturbations (g) are in this example enough to expel all spiral waves (h). Consequently, the three perturbations define the final directed shift in this example. 


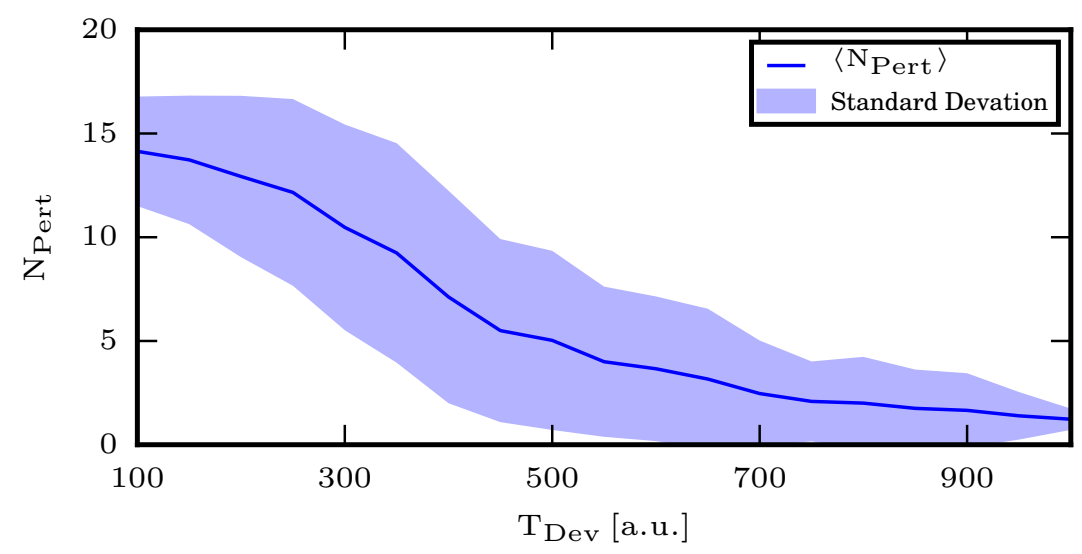

Figure 3.33: The averaged number of perturbations needed to terminate the dynamics within a given evolution time. With the algorithm discussed in this section, the number of required perturbations $\mathrm{N}_{\text {Pert }}$ to terminate the spatio-temporal dynamics was determined for all 206 chaotic episodes. In general, the longer the evolution time $\mathrm{T}_{\mathrm{Dev}}$ is, the less perturbations are needed. The statistical standard variation is sketched in light blue.

can be terminated with a minimal intervention using spatially confined perturbations of the membrane potential. $84 \%$ of the 206 episodes could be terminated with less than 10 perturbations within $\mathrm{T}_{\mathrm{Dev}}=500$ time units, which corresponds to around 5 spiral rotation. The number of single perturbations which is required to terminate the dynamics depends on the evolution time. With a larger duration between the application of the directed shift and the point in time of the desired termination, less perturbations are needed (Fig. 3.33). So far, the obtained results and analyses did not provide a sufficient understanding of the underlying mechanism of this minimal intervention approach.

\section{Summary}

In this study we investigated in two-dimensional numerical simulations of excitable media the chaotic spatio-temporal dynamics governed by spiral waves. Although we are dealing with a very high-dimensional system and the observed dynamics is highly chaotic with in average around seven spiral waves and a Kaplan-Yorke dimension of $D_{\mathrm{KY}}>60$ (Fig. 2(d) of the study in section 3.1 on page 68 , we demonstrate that the termination of the chaotic dynamics is feasible by the application of few spatially localized perturbations to the membrane potential (directed shift). Using the algorithm developed by S. P. Cornelius et al. [130, it was possible to identify proper directions in the high-dimensional state space, where corresponding perturbations lead the trajectories to a fast self-termination.

The number of required perturbations decreased with an increasing temporal duration between the application of the directed shift and the moment of the desired termination of the dynamics. Furthermore, we observed in many examples that single perturbations influence 
only a spatially limited region of the domain, which is visible in the final states during the algorithm (e.g. Fig. 3.32). This provides further information about the state space structure, and suggests to introduce a concept for the velocity information propagation concerning small scale perturbations in excitable media.

Finally, more investigations are needed in order to improve the understanding of the underlying mechanisms of this minimal intervention approach. 


\section{Chapter 4}

\section{Conclusion}

\subsection{Discussion and Outlook}

In this section, we summarize and discuss the main findings and results. At the beginning of the thesis, we emphasized that the main motivation for this work has its origins in the field of cardiac dynamics (section 1.4 on page 9). Also, we were interested to understand general features like transient dynamics in a broader and more general class of systems than the specific case of excitable media. That is, why two scientific objectives were formulated: Besides the longterm goal to benefit from a deeper insight into the governing mechanisms of ventricular fibrillation in order to develop improved clinical treatments for cardiac arrhythmias and in particular reduce the significant side-effects of current methods, the second objective aims at enhancing the understanding of chaotic transients in complex nonlinear systems on a more fundamental level.

In the following discussion, we therefore interpret the obtained results in two manners: We evaluate its significance concerning a possible influence on experimental studies and clinical applications of cardiac arrhythmias, and also highlight the role of the obtained knowledge for the general understanding of the dynamics in nonlinear systems.

\section{Features of Chaotic Transients}

We investigated diverse features of chaotic transients in excitable media in the study of section 3.1. The observation of chaotic dynamics with a finite duration in two models and using different sets of parameters underlines the robustness of the transient property. The average lifetime $\langle T\rangle$ is a characteristic quantity of the transient dynamics, which, interpreted as the length of cardiac arrhythmias, can also be highly relevant in practice.

With the exponential scaling of $\langle T\rangle$ with the system size in two dimensions, we could assign the chaotic transients in the investigated excitable systems to the group of type-II supertransients, which are characterized by an abrupt collapse of the chaotic dynamics. With the detailed investigation of the statistics of spiral waves in the system, we could show that the average number of spiral waves which fit into a simulation domain of a certain size determines the average transient duration. The permanent creation and annihilation of spiral waves could be identified as the underlying mechanism, and thus the dynamics 
of the system concerning the number of present spiral waves can be modeled as a Markov chain. From this point of view, the system has a certain probability to reach the state of zero spiral waves present, which is equivalent to self-termination. These insights into the governing processes extend the understanding of chaotic spiral wave dynamics and provide the basis for further investigations of transient dynamics in nonlinear systems.

Regarding spatio-temporal chaos during cardiac arrhythmias, the distinction between sustained and transient dynamics may be crucial. The average lifetime of chaotic episodes $\langle T\rangle$ needs to be compared to the time span $\tau_{\text {Survive }}$ a patient can survive while suffering e.g. ventricular fibrillation. Taking into account that irregularities of the heart dynamics do appear also in "healthy" hearts [132], the difference between a heart which promotes the occurrence of long living ventricular fibrillation, and a "healthy" heart is the individual average lifetime of chaotic episodes of the respective heart. Whereas, in the latter case, chaotic episodes are mostly too short to be perceptible, in "diseased" hearts $\langle T\rangle$ is of the order of $\tau_{\text {Survive }}$. An increased average lifetime of chaotic dynamics can hence be related to anatomical changes of the heart muscle. In fact, this interpretation agrees with studies which indicate that larger heart muscle volumes increase the risk of cardiac arrhythmias [133, 134, 135].

This study provides just the fundamental basis for such considerations. In a next step, the average lifetime should be determined in three-dimensional simulations using realistic geometries in order to investigate how anatomical changes (e.g. an increase of the thickness of the ventricular wall, or regions of infarct tissue) influence the average transient lifetime. Also, anisotropy features of the electrical conductivity should be taken into account here.

The interpretation of the results opens the path for novel techniques for a patient specific risk stratification. This could be based on, for example, computed tomography scans (CT scans) or magnetic resonance imaging (MRI) for detailed geometrical information. Furthermore, diffusion tensor magnetic resonance imaging (DTMRI) could be used to extract knowledge about the fiber direction of the patient's heart. Here, the impact of anatomical changes of the cardiac substrate onto the lifetime of cardiac arrhythmias can be investigated. Also, numerical simulations based on patient specific heart geometries could provide valuable information about the average lifetime of chaotic transients, and thus the danger for longliving fibrillation. The critical thickness of $\langle T\rangle$ found in three-dimensional simulations could actually play a major role here.

Furthermore, the administration of certain antiarrhythmic agents are in agreement with these considerations: antiarrhythmic agents of class-III, for example, prolong the repolarization and in this way extend the action potential duration. With an increase of the action potential duration, the average number of spiral waves in a domain of a fixed size is reduced, which can be interpreted as an effective reduction of the domain size and results in a significantly reduced transient lifetime [136.

\section{The Terminal Transient Phase of Chaotic Transients}

The actual process of self-termination as the end of chaotic episodes was the research object in the study of section 3.2 . 
We characterized the structure of the state space concerning the average lifetime in six systems from different scientific fields (cardiac dynamics, neuronal firing activity, chemical reactions, low-dimensional maps) which exhibited diverse dynamics of various levels of complexity. The state space was probed using small but finite perturbations of typical trajectories, and the subsequent determination of the lifetimes of the perturbed trajectories. Averaging the lifetimes of the perturbed trajectories, which originate from the neighborhood of the reference trajectory, provided information about a small state space volume around the perturbed state.

With this method, we could verify the existence of a transition zone in the state space between the chaotic dynamics and the final attractor (resting state), which exhibits a qualitatively different structure in the state space in comparison to the chaotic dynamics far away from self-termination. This transition zone has been verified in each system, and quantified by the duration which typical trajectories need to propagate through this zone, called Terminal Transient Phase (TTP). In all investigated systems, the TTP is significant in relation to the intrinsic time scale of the underlying dynamics (e.g. a spiral period).

The mechanism for this behavior could be identified by the emergence of subspaces in the space of possible perturbations, where perturbing the original trajectory in the corresponding direction does not cause an exponential separation from the reference trajectory, as expected in a chaotic system. Furthermore, the perturbed trajectory follows the original one to the upcoming self-termination. We found, that the directions in the subspace of perturbations form connected clusters which grow in time. This behavior motivates the picture of high-dimensional tube-like structures which guide trajectories out of the chaotic dynamics.

With the detection of the transition zone quantified by the Terminal Transient Phase, we significantly extend the general understanding of transient chaos. In particular, this contradicts the previous view that specifically in type-II supertransients the collapse of transient chaotic dynamics is abrupt and can not be predicted. In fact, with this study we show that in principle precursors should exist which, based on the different properties of the state space structure of the transition zone, could indicate the upcoming collapse. We already verified the existence of such observables in the investigated low-dimensional maps. For complex, high-dimensional systems, this remains as a major task for future studies, which could employ techniques from machine learning and data assimilation, since we actually showed in our study that trajectories are, before self-termination, robust to small perturbations which is an essential advantage for a reliable prediction of chaotic dynamics.

In the field of cardiac arrhythmias such an observable could predict a possible upcoming selftermination, and thus it could prevent the application of an unnecessary defibrillation shock and its side-effects. Also, a characterization of the states which collapse in a reasonable amount of time could be valuable here. One can think of a defibrillation protocol which perturbs the system to these states, and achieve in this way the (self-)termination of the arrhythmia.

Since transient chaotic dynamics also plays a role in other fields e.g. in the information processing of the brain (neuronal activity [110]) or in fluid dynamics [36], the existence of 
a Terminal Transient Phase could improve the understanding and control of the dynamics also there.

\section{Complexity Fluctuations During Cardiac Arrhythmia}

Clinical observations of fluctuations of the complexity or regularity of the signal from an electrocardiogram (ECG) during episodes of ventricular fibrillation provided the main motivation for the study of section 3.3 .

We quantified the complexity fluctuations of experimentally measured ECGs by determining the Permutation Entropy [119] (by Alexander Schlemmer). These fluctuations could in a first approach be reproduced by performing numerical simulations of ventricular fibrillation in a realistic rabbit heart geometry. We exploited the advantage of simulations, that the full state of the system is accessible, unlike it is the case in, for example, ex-vivo experiments of animal hearts, where the excitation patterns are mainly measurable on the surface of the heart only. By exploiting the full information available in simulations, we could demonstrate that the variations of the regularity in the measured ECG is caused by complexity fluctuations of the spatio-temporal dynamics, quantified by the number of scroll waves. We could characterize "laminar" states of the heart, where the dynamic is more organized (less scroll waves) and "complex" states, governed by a larger number of scroll waves.

After illustrating how the complexity fluctuations in the spatio-temporal dynamics causes specific patterns in the ECG time series, we investigated whether a prediction of the level of complexity of the spatio-temporal dynamics is possible, based on the ECG time series. We showed, that a single ECG electrode is not sufficient in order to make a reliable statement concerning the global spatio-temporal state, but already five electrodes provide a reasonable correlation.

Furthermore, in experimental studies (performed by Tariq Baig, Sebastian Berg, Rabea Hinkel, Daniel Hornung and Claudia Richter) using an eight-electrode setup (developed by Laura Diaz Rodriguez) we could observe similar Permutation Entropy patterns as we did in the numerical study, indicating that the hypothesis that a statement about the spatiotemporal level of complexity of the bulk of the tissue can be made using multiple ECG time series is also valid in experiments.

Since there are indications, that regarding the termination of ventricular fibrillation the defibrillation threshold (thus the required energy for a successful defibrillation) depends on the spatio-temporal level of complexity [129, the obtained knowledge of this study can provide the basis for a significant reduction of the defibrillation energy. For example, the timing of the defibrillation shock could be adjusted to a "laminar" phase (determined by a multiple-ECG measurement), where a successful termination can possibly be achieved by using less energy.

Furthermore, since it is known that defibrillation energies required for a successful cardioversion are particularly different between tachycardia and ventricular fibrillation [137, it is of great interest whether a "laminar" phase during fibrillation is dynamically comparable to monomorphic or polymorphic tachycardia. 
Also, our study revealed that with a multiple-ECG setup one can not only estimate the global level of complexity of the heart. The multiple time series provide even information about the spatial localization of scroll waves. This knowledge opens the path for a state specific defibrillation scheme, where, for example, different defibrillation vectors (thus the direction of the electrical field vector) could be used, each for specific dynamical states and distributions of scroll waves.

These considerations demand extensive numerical and experimental studies in the future. However, with the identification and characterization of the fundamental mechanism, namely the correlation between the spatio-temporal dynamics and multiple-ECG time series, we establish the basis for ongoing studies.

\section{Termination via Perturbation}

Based on the obtained knowledge about the structure of the state space in previous studies, the study of section 3.4 deals with the active termination of chaotic dynamics. The obtained results represent mainly a proof of concept of a novel approach to terminate high-dimensional spatio-temporal chaos using minimal intervention.

We showed that the investigated complex chaotic dynamics in high-dimensional systems can be terminated by the application of state specific arrangements of small and spatially limited perturbations. A termination with such a minimal interference with the system is possible only with the proper set of perturbations which add up to a "directed shift" which pushes the trajectory to the collapse of the dynamics. In order to find the proper combination of local perturbations in the high-dimensional space of possible perturbations, we used the algorithm developed by S. P. Cornelius et al. [130].

During the algorithm it is striking that by adding new single perturbations to the "directed shift" does not cause a total change of the final state. Rather, in many cases one can observe that adding a single perturbation terminates particular spiral waves in the final state without changing the rest of the state. This observation needs to be investigated intensively in the future, but already emphasizes that a state specific defibrillation technique should be taken into account for the development of novel defibrillation techniques. Furthermore, this behavior is also an indication that the complex spatio-temporal dynamics of the investigated systems can be described by spatially extended and temporally only weakly interacting "tiles", defined by the spiral waves as proposed by Byrne et al. [131.

Furthermore, the algorithm for the determination of the directed shift is extremely elaborate, and demanding in terms of computational time. Thus it is desirable to develop a simple and robust "recipe" for the proper arrangement of single perturbations on the basis of the present results. This concept could furthermore be tested in three-dimensional simulations, also under the constraint that in a realistic geometry, for example, only the outer surface can be perturbed. Such a hypothetical concept could then also influence ideas for completely novel experimental and clinical defibrillation techniques. 


\subsection{Concluding Remarks}

As a general conclusion, we hope that the insights provided by this thesis contribute to the general understanding of cardiac arrhythmias and the nonlinear dynamics of complex systems. The results suggest that an improved medical treatment of cardiac arrhythmias can benefit from:

- A more detailed state analysis of the dynamics during spatio-temporal chaos, incorporating diverse measure techniques (e.g. multiple-ECG measurements, CT scans, MRI scans).

- An intervention strategy which should adapt to individual patients and the respective dynamical state of the heart.

A variety of new experimental approaches will be available which may help to achieve these goals and to improve the understanding of the phenomena investigated in this thesis: Filament identification in the bulk tissue during experiments using sophisticated ultra sound techniques, inverse ECG measurements for the reconstruction of spatio-temporal wave dynamics or using techniques from optogenetics for the stimulation of cardiac tissue via light pulses are promising candidates which can have a significant impact on the field of cardiac dynamics.

This technological progress in combination with novel data analysis techniques from the fields of machine learning or data assimilation and sophisticated simulations of the complex dynamics has great potential to develop advanced and efficient strategies for a patient specific medical treatment.

This thesis shows how concepts from nonlinear dynamics can provide valuable insights into fields like cardiac dynamics. For this reason also fundamental and rather mathematical considerations, which do not have direct practical implications, can contribute to the understanding of complex biological systems on the long-term. 


\section{Appendix A}

\section{Models of Cardiac Cell Dynamics}

\section{A.1 Aliev-Panfilov Model}

\section{Parameter Sets}

Table A.1 presents the parameters used for simulations of Aliev-Panfilov model.

Table A.1: The parameter set AP1, used for simulations with the Aliev-Panfilov model.

\begin{tabular}{cccc}
\hline & Value [a.u.] & & Value [a.u.] \\
\hline $\mathrm{k}$ & 8 & $\mu_{1}$ & 0.2 \\
$\mathrm{a}$ & 0.05 & $\mu_{2}$ & 0.3 \\
$\epsilon_{0}$ & 0.002 & & \\
\hline
\end{tabular}




\section{A.2 Fenton-Karma Model}

\section{Parameter Sets}

Table A.2 - A.4 present the parameters used for simulations of Fentom-Karma model (taken from [74]).

Table A.2: The parameter set FK1, used for simulations with the Fenton-Karma model.

\begin{tabular}{cclc}
\hline & Value [a.u.] & & Value [a.u.] \\
\hline$\tau_{\mathrm{v}+}$ & 13.03 & $\tau_{0}$ & 12.5 \\
$\tau_{\mathrm{v} 1}^{-}$ & 19.6 & $\tau_{\mathrm{r}}$ & 33.25 \\
$\tau_{\mathrm{v} 2}^{-}$ & 1250 & $\tau_{\mathrm{si}}$ & 29 \\
$\tau_{\mathrm{w}}^{+}$ & 800 & $\mathrm{u}_{\mathrm{c}}$ & 0.13 \\
$\tau_{\mathrm{w}}^{-}$ & 40 & $\mathrm{u}_{\mathrm{c}}^{\mathrm{si}}$ & 0.85 \\
$\tau_{\mathrm{d}}$ & 0.45 & $\mathrm{u}_{\mathrm{v}}$ & 0.04 \\
$\mathrm{C}_{\mathrm{m}}$ & 1 & $\mathrm{k}$ & 500 \\
\hline
\end{tabular}

Table A.3: The parameter set FK2, used for simulations with the Fenton-Karma model.

\begin{tabular}{cclc}
\hline & Value [a.u.] & & Value [a.u.] \\
\hline$\tau_{\mathrm{v}+}$ & 3.33 & $\tau_{0}$ & 12.5 \\
$\tau_{\mathrm{v} 1}^{-}$ & 15 & $\tau_{\mathrm{r}}$ & 28 \\
$\tau_{\mathrm{v} 2}^{-}$ & 2 & $\tau_{\mathrm{si}}$ & 29 \\
$\tau_{\mathrm{w}}^{+}$ & 670 & $\mathrm{u}_{\mathrm{c}}$ & 0.13 \\
$\tau_{\mathrm{w}}^{-}$ & 61 & $\mathrm{u}_{\mathrm{c}}^{\mathrm{si}}$ & 0.45 \\
$\tau_{\mathrm{d}}$ & 0.25 & $\mathrm{u}_{\mathrm{v}}$ & 0.05 \\
$\mathrm{C}_{\mathrm{m}}$ & 1 & $\mathrm{k}$ & 500 \\
\hline
\end{tabular}

Table A.4: The parameter set FK3, used for simulations with the Fenton-Karma model.

\begin{tabular}{cclc}
\hline & Value [a.u.] & & Value [a.u.] \\
\hline$\tau_{\mathrm{v}+}$ & 3.33 & $\tau_{0}$ & 9 \\
$\tau_{\mathrm{v} 1}^{-}$ & 15.6 & $\tau_{\mathrm{r}}$ & 34 \\
$\tau_{\mathrm{v} 2}^{-}$ & 5 & $\tau_{\mathrm{si}}$ & 26.5 \\
$\tau_{\mathrm{w}}^{+}$ & 350 & $\mathrm{u}_{\mathrm{c}}$ & 0.15 \\
$\tau_{\mathrm{w}}^{-}$ & 80 & $\mathrm{u}_{\mathrm{c}}^{\text {si }}$ & 0.45 \\
$\tau_{\mathrm{d}}$ & 0.25 & $\mathrm{u}_{\mathrm{v}}$ & 0.04 \\
$\mathrm{C}_{\mathrm{m}}$ & 1 & $\mathrm{k}$ & 500 \\
\hline
\end{tabular}




\section{A.3 Bueno-Orovio-Cherry-Fenton Model}

\section{Differential Equations}

The equations for the ionic currents can be found in Eq. A.1 - A.3. Equations A.4 A.6 show the evolution equations of the gating variables $v, w$ and $s$ of the Bueno-OrovioCherry-Fenton model, whereas Eqs. A.7 - A.13 display additional definitions and expressions used in the model:

$$
\begin{gathered}
I_{\mathrm{fi}}(u, v)=-\frac{v}{\tau_{f i}} \Theta\left(u-\theta_{v}\right)\left(u-\theta_{v}\right)\left(u_{u}-u\right), \\
I_{\mathrm{so}}(u)=\frac{u-u_{0}}{\tau_{o}}\left(1-\Theta\left(u-\theta_{w}\right)\right)+\frac{\Theta\left(u-\theta_{w}\right)}{\tau_{s o}}, \\
I_{\mathrm{si}}(u, w, s)=-\frac{w s}{\tau_{s i}} \Theta\left(u-\theta_{w}\right) . \\
\frac{\partial v}{\partial t}=\left[1-\Theta\left(V_{m}-\theta_{v}\right)\right] \frac{\left(v_{\infty}-v\right)}{\tau_{v}^{-}}-\Theta\left(V_{m}-\theta_{v}\right) \frac{v}{\tau_{v}^{+}}, \\
\frac{\partial w}{\partial t}=\left[1-\Theta\left(V_{m}-\theta_{w}\right)\right] \frac{\left(w_{\infty}-w\right)}{\tau_{w}^{-}}-\Theta\left(V_{m}-\theta_{w}\right) \frac{w}{\tau_{w}^{+}}, \\
\frac{\partial s}{\partial t}=\left(\frac{1+\tanh \left[k_{s}\left(V_{m}-u_{s}\right)\right]}{2}-s\right) / \tau_{s}, \\
\tau_{v}^{-}=\left[1-\Theta\left(V_{m}-\theta_{v}^{-}\right)\right] \tau_{v 1}^{-}+\Theta\left(V_{m}-\theta_{v}^{-}\right) \tau_{v 2}^{-}, \\
\tau_{w}^{-}=\tau_{w 1}^{-}+\frac{\tau_{w 2}^{-}-\tau_{w 1}^{-}}{2}\left[1+\tanh \left(k_{w}^{-}\left(V_{m}-u_{w}^{-}\right)\right)\right] \\
\tau_{s o}=\tau_{s o 1}+\frac{\tau_{s o 2}-\tau_{s o 1}}{2}\left[1+\tanh \left(k_{s o}\left(V_{m}-u_{s o}\right)\right)\right] \\
\tau_{s}=\left[\begin{array}{ll}
\left.1-\Theta\left(V_{m}-\theta_{w}\right)\right] \tau_{s 1}+\Theta\left(V_{m}-\theta_{w}\right) \tau_{s 2}, \\
\tau_{o}=
\end{array}\right. \\
{\left[1-\Theta\left(V_{m}-\theta_{o}\right)\right] \tau_{o 1}+\Theta\left(V_{m}-\theta_{o}\right) \tau_{o 2},} \\
V_{m} \geq \theta_{v}^{-}, \\
{\left[1-\Theta\left(V_{m}-\theta_{o}\right)\right)\left(1-\frac{V_{m}}{\tau_{w \infty}}\right)+\Theta\left(V_{m}-\theta_{o}\right) w_{\infty}^{*}}
\end{gathered}
$$




\section{Parameter Sets}

Table A.5 presents the parameters used for simulations of the Bueno-Orovio-Cherry-Fenton model (taken from [90]).

Table A.5: The parameter set BOCF1, used for simulations of the Bueno-Orovio-CherryFenton model.

\begin{tabular}{lclclc}
\hline & Value [a.u.] & & Value [a.u.] & & Value [a.u.] \\
\hline $\mathrm{u}_{0}$ & 0 & $\tau_{\mathrm{fi}}$ & 0.11 & $\mathrm{u}_{\mathrm{u}}$ & 1.58 \\
$\tau_{\mathrm{o} 1}$ & 6 & $\theta_{\mathrm{v}}$ & 0.3 & $\tau_{\mathrm{o} 2}$ & 6 \\
$\theta_{\mathrm{w}}$ & 0.015 & $\tau_{\mathrm{so} 1}$ & 43 & $\theta_{\mathrm{v}}^{-}$ & 0.015 \\
$\tau_{\mathrm{so} 2}$ & 0.2 & $\theta_{\mathrm{o}}$ & 0.006 & $\mathrm{k}_{\mathrm{so}}$ & 2 \\
$\tau_{\mathrm{v} 1}^{-}$ & 60 & $\mathrm{u}_{\mathrm{so}}$ & 0.65 & $\tau_{\mathrm{v} 2}^{-}$ & 1150 \\
$\tau_{\mathrm{s} 1}$ & 2.7342 & $\tau_{\mathrm{v}}^{+}$ & 1.4506 & $\tau_{\mathrm{s} 2}$ & 3 \\
$\tau_{\mathrm{w} 1}^{-}$ & 70 & $\mathrm{k}_{\mathrm{s}}$ & 2.0994 & $\tau_{\mathrm{w} 2}^{-}$ & 20 \\
$\mathrm{u}_{\mathrm{s}}$ & 0.9087 & $\mathrm{k}_{\mathrm{w}}^{-}$ & 65 & $\tau_{\mathrm{si}}$ & 2.8723 \\
$\mathrm{u}_{\mathrm{w}}^{-}$ & 0.03 & $\tau_{\mathrm{w} \infty}$ & 0.07 & $\tau_{\mathrm{w}}^{+}$ & 280 \\
$\mathrm{w}_{\infty}^{*}$ & 0.94 & & & & \\
\hline
\end{tabular}




\section{Appendix B}

\section{Supplemental Material}

B.1 Supplemental Material 1: Features of Chaotic Transients in Excitable Media

In this section, Supplemental Material for the study "Features of Chaotic Transients in Excitable Media Governed by Spiral and Scroll Waves" are given. 


\section{Supplemental Material}

\section{DETAILED EQUATIONS OF THE FENTON-KARMA MODEL}

The equations (1) - (2) describe the evolution of the variables $v$ and $w$ of the Fenton-Karma model.

$$
\begin{aligned}
\frac{\partial v}{\partial t} & =\Theta\left(u_{c}-u\right)(1-v)\left(\frac{\Theta\left(u-u_{v}\right)}{\tau_{v 1}^{-}}+\frac{\Theta\left(u_{v}-u\right)}{\tau_{v 2}^{-}}\right)-\Theta\left(u-u_{c}\right) \frac{v}{\tau_{v}^{+}} \\
\frac{\partial w}{\partial t} & =\Theta\left(u_{c}-u\right) \frac{1-w}{\tau_{w}^{-}}-\Theta\left(u-u_{c}\right) \frac{w}{\tau_{w}^{+}}
\end{aligned}
$$

The term for the ionic currents $I_{I o n}$ (Eq. (3)) in the Fenton-Karma model has three contributions: the fast inward current $J_{f i}$, the slow outward current $J_{s o}$ and the slow inward current $J_{s i}$. They are shown in Eqs. (4) - (6).

$$
\begin{aligned}
I_{\text {Ion }}(u, v, w) & =-J_{f i}(u, v)-J_{s o}(u)-J_{s i}(u, w), \\
J_{f i}(u, v) & =-\frac{v}{\tau_{d}} \Theta\left(u-u_{c}\right)(1-u)\left(u-u_{c}\right), \\
J_{s o}(u) & =\frac{u}{\tau_{0}} \Theta\left(u_{c}-u\right)+\frac{1}{\tau_{r}} \Theta\left(u-u_{c}\right), \\
J_{s i}(u, w) & =-\frac{w}{2 \tau_{s i}}\left(1+\tanh \left[k\left(u-u_{c}^{s i}\right)\right]\right) .
\end{aligned}
$$

\section{PARAMETER SETS}

Table I and Table II show the parameter sets used for simulations using the Aliev-Panfilov model (AP) and the Fenton-Karma model (FK1 and FK2), respectively.

TABLE I. Parameter set used for simulations with the AlievPanfilov model: (AP).

\begin{tabular}{lccc}
\hline \hline & Value [a.u.] & & Value [a.u.] \\
\hline $\mathrm{k}$ & 8 & $\mu_{1}$ & 0.2 \\
$\mathrm{a}$ & 0.05 & $\mu_{2}$ & 0.3 \\
$\epsilon_{0}$ & 0.002 & & \\
\hline \hline
\end{tabular}

TABLE II. Two parameter sets used for the simulations with the Fenton-Karma model: parameter set 1 (FK1) and parameter set 2 (FK2).

\begin{tabular}{lclllc}
\hline \hline & FK1 & FK2 & & FK1 & FK2 \\
\hline$\tau_{\mathrm{v}+}$ & 13.03 & 3.33 & $\tau_{0}$ & 12.5 & 12.5 \\
$\tau_{\mathrm{v} 1}^{-}$ & 19.6 & 15 & $\tau_{\mathrm{r}}$ & 33.25 & 28 \\
$\tau_{\mathrm{v} 2}^{-}$ & 1250 & 2 & $\tau_{\mathrm{si}}$ & 29 & 29 \\
$\tau_{\mathrm{w}}^{+}$ & 800 & 670 & $\mathrm{u}_{\mathrm{c}}$ & 0.13 & 0.13 \\
$\tau_{\mathrm{w}}^{-}$ & 40 & 61 & $\mathrm{u}_{\mathrm{c}}^{\mathrm{si}}$ & 0.85 & 0.45 \\
$\tau_{\mathrm{d}}$ & 0.45 & 0.25 & $\mathrm{u}_{\mathrm{v}}$ & 0.04 & 0.05 \\
$\mathrm{C}_{\mathrm{m}}$ & 1 & 1 & & & \\
\hline \hline
\end{tabular}

\section{THE INDUCTION OF CHAOTIC DYANMICS}

Chaotic dynamics was induced in all simulations by giving several local stimuli along the boundary of one edge of the grid, synchronized with the wave tail of a plane wave which propagates perpendicular to this edge. Due to differences in the initial timing of these additional pulses, distinct initial conditions were created. Due to the chaotic nature of the dynamics (positive Lyapunov exponents), initial conditions which were created by stimuli only separated by a small amount of time, separated from each other exponentially fast. Figure 1 depicts the course of such an induction process exemplary for a simulation using the FK1 parameter set (Fenton-Karma model).

\section{THE DETECTION OF PHASE SINGULARITIES AND FILAMENTS}

Phase singularities and filaments were detected in all simulations by first calculating the phase by a pointwise Hilbert transformation. Phase singularities were then identified, by performing a closed line integral (surface integral in 3D) of the phase at every point of the simulation domain. In $2 \mathrm{D}$, this resulted in a certain number of phase singularities, which can be associated with the spiral waves. In three dimensional simulations, the cor- 

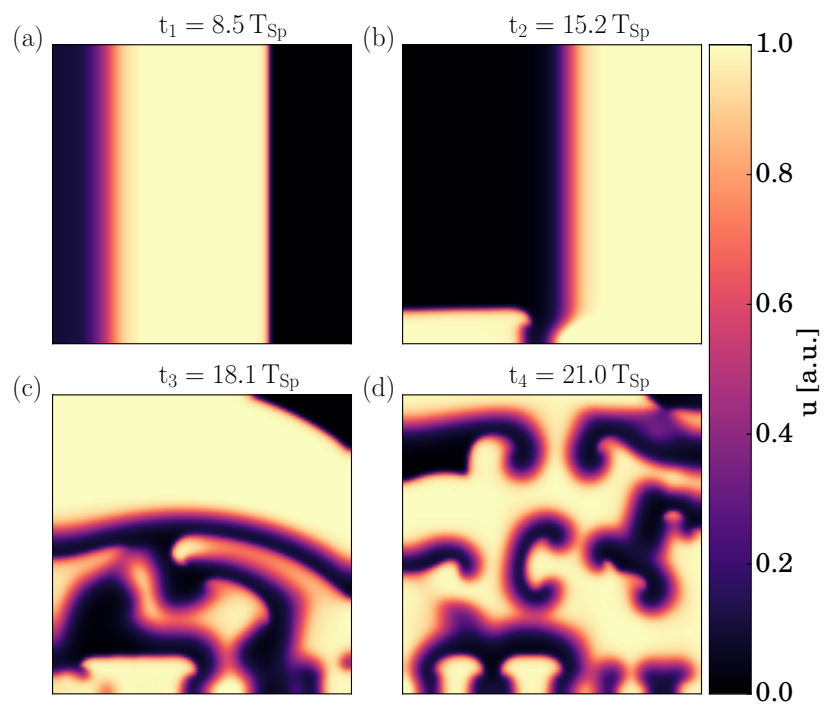

FIG. 1. Snapshots of the membrane potential variable $u$ at different instances of time during an exemplary induction process using the Fenton-Karma model and parameter set FK1. Initially, plane waves are induced at one edge of the tissue ((a), propagating from left to right). Local stimuli were then given at the edge perpendicular to the propagation direction of the initial plane wave (lower part in (b)). By the additional stimuli, spiral waves were created and additional wave breakups lead to spatio-temporal chaos ((c) and (d)). Time is given in spiral rotations $\left(\mathrm{T}_{\mathrm{Sp}}\right)$.

responding objects which describe the core of a scroll wave are one dimensional curve like filaments. In order to merge related phase singularities to the corresponding filament, they were identified with the same filament if the euclidean distance was below $2 \times \sqrt{2}$ grid points.

\section{RESULTS CONCERNING THE IMPACT OF NOISE ON THE LIFETIME OF CHAOTIC TRANSIENTS}

We also investigated the effect of noise and applied gaussian white noise to the diffusive dynamical variable of the systems. This was done by adding $\sigma W_{t}$ to the evolution equation of the membrane potential variable $u$ at every node of the domain. In Eq. (7), $f(u)$ denotes the local cell dynamics (Aliev-Panfilov model or Fenton-Karma model, respectively), whereas $\sigma$ is the noise amplitude and $W_{t}$ standard normal distributed random numbers.

$$
\frac{\partial u}{\partial t}=\nabla \cdot \underline{\mathbf{D}} \nabla u+f(u)+\sigma W_{t} .
$$

If the noise amplitude is above a certain level, excitation waves are generated because noise induced fluctuations are strong enough to locally exceed the excitation threshold. Hence, with a high noise amplitude, the system does not terminate by itself anymore. Here, we want to study how noise changes the spatio-temporal dynamics without additional noise induced excitations, thus we want to stay in a low noise amplitude regime, where noise induced bursts of not excited tissue can be neglected. In a first study, noise was added to not excited tissue (in all three models) for a simulation time equivalent to 50.000 spiral rotations. For various noise amplitudes, the number of noise induced excitation spikes during this interval was counted. Since a local spike causes a global excitation wave, a spike was identified when the overall sum of excitations of the whole tissue exceeded an excitation threshold of $\left(\frac{1}{L_{x} \times L_{y}} \sum_{i, j} u_{i j}>0.5\right)$. We found that in an amplitude range of $\sigma<0.02$ the abovementioned effect of noise induced bursts is not present and does not affect our investigations (Fig. 2(a)).

Figure 2(b) shows that noise can have different impacts on the average transient lifetime: although the lifetime increases generally with increasing noise amplitude in a low amplitude regime, the behavior is not necessarily monotone. Also, noise affects the increase in lifetime differently in each cell model or for each parameter set.
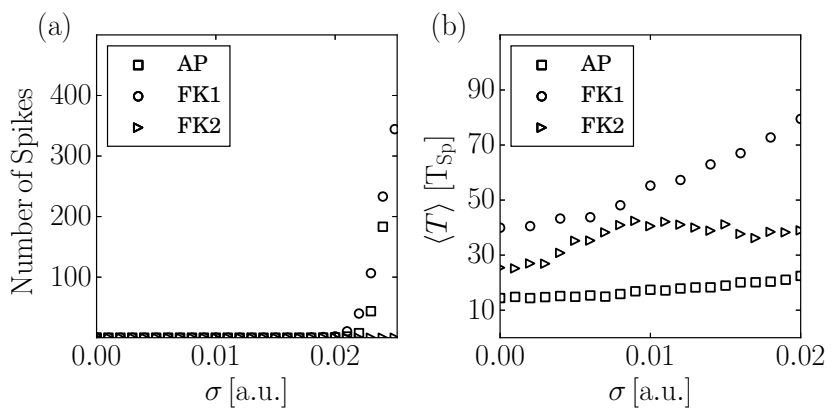

FIG. 2. The impact of the addition of gaussian white noise to the system. In subplot (a) the number of excitation spikes is plotted depending on the noise amplitude $\sigma$ for AP $(\square)$, FK1 $(\circ)$ and FK2 $(\triangleright)$. Subplot (b) shows the impact of noise of increasing amplitude $\sigma$ on the average transient lifetime $\langle T\rangle$ for AP ( $\square)$, FK1 (०) and FK2 ( $)$ )).

The addition of noise could be interpreted here as a model for cell variability. Noise changes effectively the propagation of phase singularities and has an influence on the creation and annihilation of spiral waves, the main governing mechanisms of the self termination of the dynamics. Furthermore in terms of the control of chaotic transients, the result shows that the application of a generic (thus not state specific) signal can not decrease the average transient lifetime (at least in the used models and white noise). Concerning applications like cardiac arrhythmias this means that finding such a generic signal which leads to a significant reduction of transient lifetimes, whereas letting the usual, desired dynamics untouched (ordinary pumping function), could be a relevant and valuable objective in the future. 


\section{DETAILS DETERMINATION OF THE LYAPUNOV SPECTRUM}

The Lyapunov spectrum was determined in $2 \mathrm{D}$ simulations (local cell parameters from FK1 with $h=1.0$ and $d t=0.5$ ), carrying along initially small but finite perturbations, and extracting the local Lyapunov exponents every 5 time units. The calculation was stopped around 5 spiral rotations before self termination. This analysis was done for various domain sizes. From 100 initial conditions, those with a transient longer than 30 spiral rotations were used in order to calculate the mean Lyapunov exponents for each domain size respectively (in order to make sure that the estimates of the Lyapunov exponents converged sufficiently).

\section{TIME SERIES OF EXEMPLARY QUANTITIES BEFORE SELF TERMINATION}

We found that self termination of the spatio-temporal chaotic dynamics in the simulations comes abrupt rather than being a continuous process which can be detected already a long time before the actual collapse. As an example, Fig. 3 shows time series of two quantities commonly used to describe the development of such a system for an exemplary simulation using the FK1 model on a two dimensional $L_{x} \times L_{y}=100 \times 100$ domain. Figure 3(a) depicts the pseudo ECG (Electrocardiogram) before the self termination (around $t \approx 125 \mathrm{~T}_{\mathrm{Sp}}$ ), which was determined as the mean excitability integrated over the simulation domain $\left(E C G \approx \frac{1}{L_{x} \times L_{y}} \sum_{i, j} u_{i j}\right)$. The number of phase singularities (thus, the number of spiral waves) $N_{P S}$ is depicted in Fig. 3(b). Both quantities fluctuate during the chaotic episode, but the upcoming collapse is not obvious.

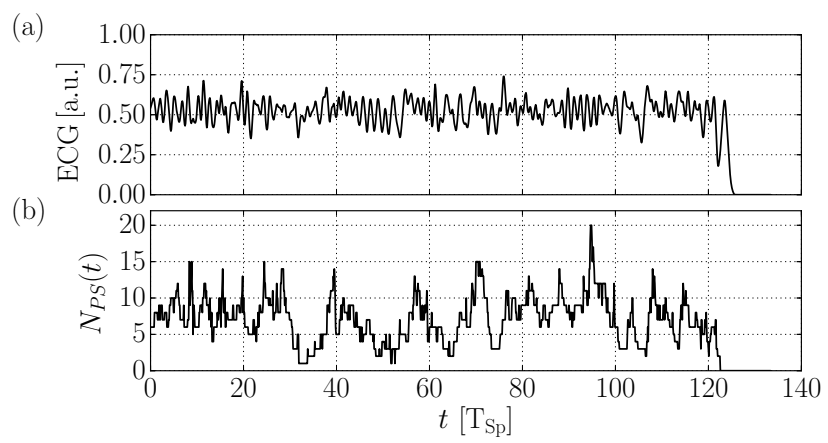

FIG. 3. Pseudo ECG and the number of phase singularities $N_{P S}$ for an exemplary episode of spatio-temporal chaotic dynamics using the FK1 on a two dimensional $L_{x} \times L_{y}=$ $100 \times 100$ domain. The chaotic episode ends by self termination at around $t \approx 125 \mathrm{~T}_{\mathrm{Sp}}$. 

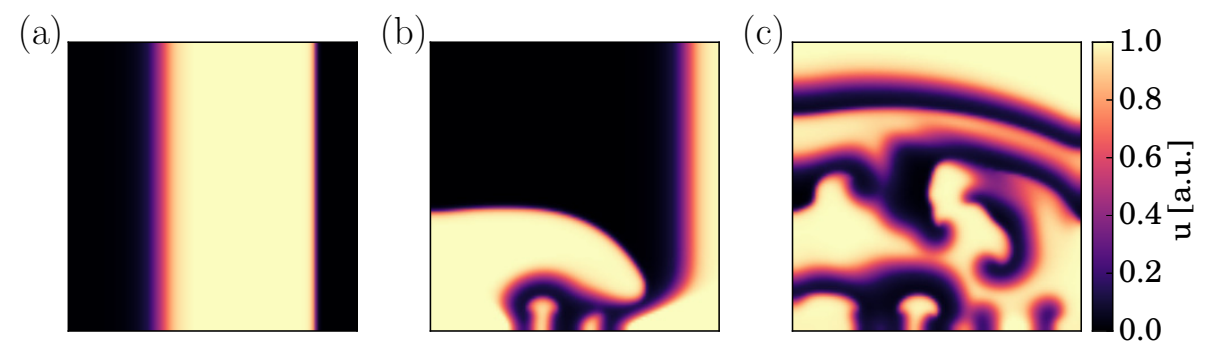

Figure B.1: Creation of an exemplary chaotic initial condition of simulations using the Fenton-Karma model. Snapshots of the membrane potential variable $u$ are shown at three points in time. Stimuli along the left boundary of the simulation domain create a plane wave propagating to the right boundary of the domain (a). Afterwards, local stimuli at the lower boundary create spiral waves (b) and initiate spatio-temporal chaos which spreads finally over the whole domain (c).

\section{B.2 Supplemental Material 2: Terminal Transient Phase of Chaotic Transients}

\section{B.2.1 Fenton-Karma Model}

\section{Creation of Chaos and Detection of Termination}

Chaotic dynamics was induced by a cross pacing protocol. Initially, several local stimuli were given along one edge of the two-dimensional simulation domain, resulting in plane waves (Fig. B.1). Afterwards, stimuli along the edge perpendicular to the propagation direction of the initial plane wave creates spiral waves which then govern the spatio-temporal dynamics. With a different timing of the second stimuli, 3000 initial conditions were created.

Figure B.2 sketches the process in the two-dimensional simulations, showing snapshots of the variable $u$ of an exemplary initial condition using the Fenton-Karma model. The difference in time between the snapshots is equivalent to half of a spiral period $\left(\mathrm{T}_{\mathrm{Sp}}\right)$.

The collapse of the spatio-temporal dynamics was defined at that point in time when the mean of the membrane potential (variable $u$, integrated over the domain) came below a certain threshold $\left.\frac{1}{N_{x} \times N_{y}} \sum_{i, j} u_{i j}<0.001\right)$.

For the determination of $\langle\mathrm{T}\rangle_{\mathrm{IC}}$, the amount of time equal to 50 spiral rotations was discarded at the beginning.

\section{B.2.2 Bueno-Orovio-Cherry-Fenton Model}

\section{Creation of Chaos and Detection of Termination}

The creation of the initial conditions was done with a cross pacing protocol, similarly to simulations of the Fenton-Karma model (see previous section). The collapse of the chaotic dynamics was determined by a drop of the overall mean of the membrane potential $(u$ variable) below a certain threshold $\frac{1}{N_{x} \times N_{y}} \sum_{i, j} u_{i j}<0.001$. For the determination of $\langle\mathrm{T}\rangle_{\mathrm{IC}}$, the amount of time equal to 50 spiral rotations was discarded. 

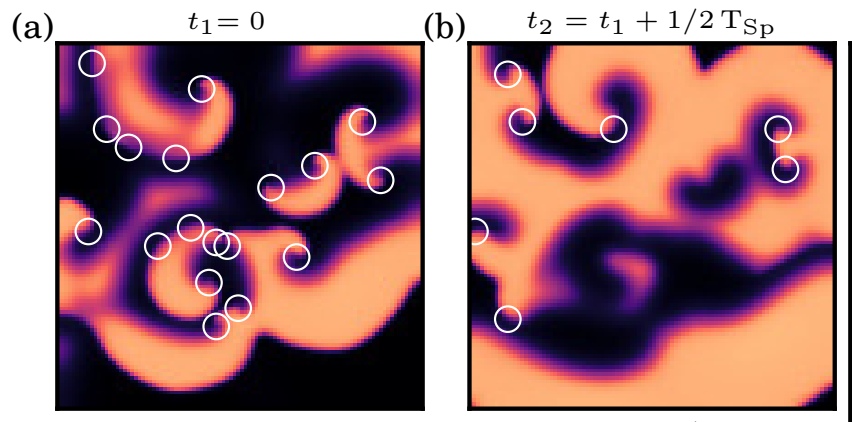

(c)

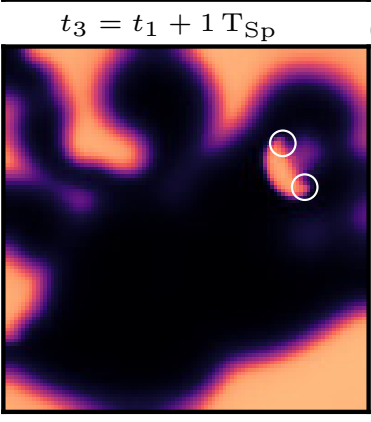

(d)
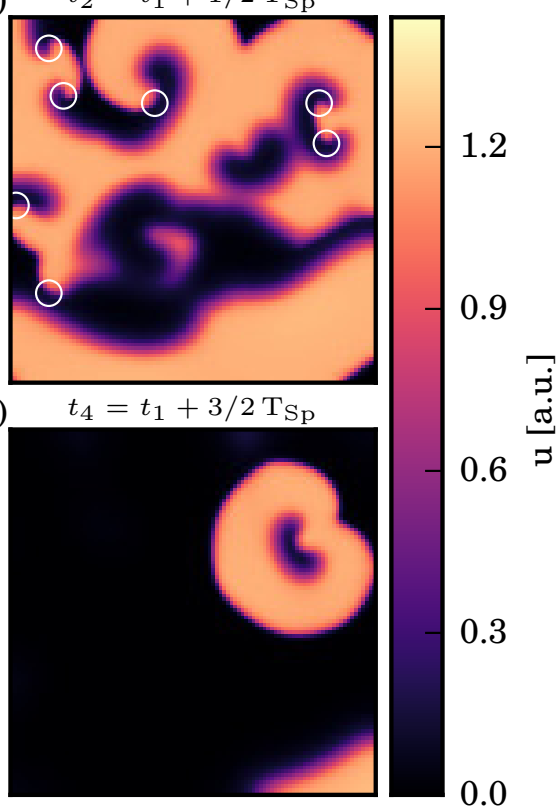

Figure B.2: An exemplary episode of self-termination of spatio-temporal chaos governed by spiral waves using the Fenton-Karma model. Snapshots of the membrane potential variable $u$ are shown at different instances of time. In subplot (a) at $t_{1}$ many phase singularities (marked by white circles) are present in the system (some phase singularities can not directly be identified as the tips of spiral waves at this instant of time. However, taking the temporal evolution into account, these phase singularities can be associated with e.g. the creation or termination process of spiral waves, or represent very short-living spiral wavelets). At time $t_{2}$ (b) some of the phase singularities have already vanished. After another time span of half a spiral period $\left((\mathrm{c}), t_{3}\right)$, only one double spiral wave has survived (marked by the two white circles). Eventually the two arms of the spiral waves collide with each other $\left((\mathrm{d}), t_{4}\right)$, annihilate and only plane waves are left over.

\section{B.2.3 Morris-Lecar neuronal ring network}

\section{Parameters}

The additional equations of the Morris-Lecar neuronal ring network are given by Eqs. (B.1) (B.6): 
Table B.1: The set of parameters used for simulations with the Morris-Lecar network.

\begin{tabular}{lclclc}
\hline & Value & & Value & & Value \\
\hline $\mathrm{C}$ & $20 \frac{\mathrm{\mu F}}{\mathrm{cm}^{2}}$ & $\mathrm{I}$ & $30 \frac{\mu \mathrm{A}}{\mathrm{cm}^{2}}$ & $\varphi$ & $1 / 15 \mathrm{~Hz}$ \\
$\mathrm{~g}_{\mathrm{L}}$ & $2 \frac{\mathrm{mS}^{2}}{\mathrm{~cm}^{2}}$ & $\mathrm{~g}_{\mathrm{K}}$ & $8 \frac{\mathrm{mS}}{\mathrm{cm}^{2}}$ & $\mathrm{~g}_{\mathrm{Ca}}$ & $4 \frac{\mathrm{mS}}{\mathrm{cm}^{2}}$ \\
$\mathrm{~V}_{\mathrm{K}}$ & $-80 \mathrm{mV}$ & $\mathrm{V}_{\mathrm{Ca}}$ & $120 \mathrm{mV}$ & $\mathrm{V}_{\mathrm{L}}$ & $-60 \mathrm{mV}$ \\
$\mathrm{V}_{1}$ & $-1.2 \mathrm{mV}$ & $\mathrm{V}_{2}$ & $18 \mathrm{mV}$ & $\mathrm{V}_{3}$ & $14.95 \mathrm{mV}$ \\
$\mathrm{V}_{4}$ & $17.4 \mathrm{mV}$ & & & & \\
\hline
\end{tabular}

$$
\begin{aligned}
I_{L}\left(V_{i}\right) & =g_{L}\left(V_{i}-V_{L}\right), \\
I_{C a}\left(V_{i}\right) & =g_{C a} m_{s s}\left(V_{i}-V_{C a}\right), \\
I_{K}\left(V_{i}, n_{i}\right) & =g_{K} n\left(V_{i}-V_{K}\right), \\
m_{s s}\left(V_{i}\right) & =\frac{1}{2}\left(1+\tanh \left[\frac{V_{i}-V_{1}}{V_{2}}\right]\right), \\
n_{s s}\left(V_{i}\right) & =\frac{1}{2}\left(1+\tanh \left[\frac{V_{i}-V_{r}}{V_{4}}\right]\right), \\
\tau_{n}\left(V_{i}\right) & =\frac{1}{\varphi \cosh \left[\frac{V_{i}-V_{3}}{2 V_{4}}\right]} .
\end{aligned}
$$

\section{Parameters}

In Tab. B.1, the parameters used for simulations of the Morris-Lecar network are presented.

\section{Creation of Chaos and Detection of Termination}

Initially, the $V$ variable was set to $-40 \mathrm{mV}$, while $n$ was set to 0 . Chaotic dynamics was initialized by setting $V$ of ten randomly chosen neurons to $-10 \mathrm{mV}$. In simulations of the Morris-Lecar network, the chaotic dynamics did not only collapse to the rest state, but also to a period pulsed state. In order to recognize if the chaotic dynamics has finished, we defined the end of the chaotic dyanmics here when the mean membrane potential (variable $V$ ) was below a threshold of $V^{\mathrm{thr}}=-39 \mathrm{mV}$ for at least $1 \mathrm{~s}$. For the determination of $\langle\mathrm{T}\rangle_{\mathrm{IC}}$, the amount of time equal to $1 \mathrm{~s}$ was discarded at the beginning.

\section{B.2.4 Gray-Scott Model}

The Gray-Scott model [92] is a simple system of partial differential equations which models a chemical reaction of the species $\mathrm{U}, \mathrm{V}$ and $\mathrm{P}: U+2 V \rightarrow 3 V, V \rightarrow P$. It can exhibit diverse irregular spatio-temporal patterns [138] and features of transient chaos have been studied by Wackerbauer et al. [95]. The model equations describe the evolution of the concentrations of the chemical species $U$ and $V$ (Eqs. (B.7) and (B.8)), where both variables are diffusive 


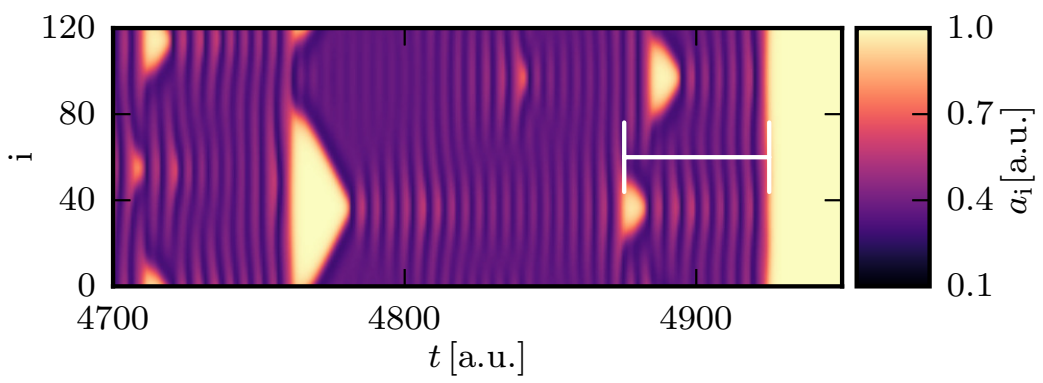

Figure B.3: A (transient) episode of spatio-temporal chaos using the Gray-Scott model. Variable $a_{i}$ is plotted color coded for the final part of the episode, just before the selftermination. The dynamics is mainly periodically (with a period of around 5-6 a.u.) interrupted by areas of high concentration of $a_{i}$. The white bar depicts the length of the Terminal Transient Phase.

Table B.2: The set of parameters used for simulations of the Gray-Scott model.

\begin{tabular}{cccccc}
\hline & Value [a.u.] & & Value [a.u.] & & Value [a.u.] \\
\hline$\mu$ & 33.7 & $\Phi$ & 2.8 & $b$ & 0 \\
\hline
\end{tabular}

(first terms, respectively).

$$
\begin{aligned}
& \frac{\partial a_{i}}{\partial t}=D \Delta a_{i}+1-a_{i}-\mu a_{i} b_{i}^{2}, \\
& \frac{\partial b_{i}}{\partial t}=D \Delta b_{i}+b_{0}-\Phi b_{i}+\mu a_{i} b_{i}^{2} .
\end{aligned}
$$

Our simulations were performed on a one-dimensional ring with $N=120$ elements, using a diffusion constant of $D=16$, a spacing constant of $h=1$ and a time constant of $d t=0.005$, where periodic boundary conditions were used. The parameters used for these simulations can be found in Tab. B.2. An example of a chaotic episode and the following self-termination of the dynamics is shown in Fig. B.3. A periodic dynamic can be observed (with a period of around 5-6 a.u.) interrupted by areas of various sizes of a high concentration (e.g around $t=4760)$ which after their appearance decrease in size and disappear.

\section{Parameters}

Table B.2 presents the parameters used for simulations using the Gray-Scott model.

\section{Creation of Chaos and Detection of Termination}

The one-dimensional simulation domain was initialized with $a=1$ and $b=0$. Chaotic dynamics was then induced by setting the $b$ variable at three blocks of each three nodes to one. The three blocks had a minimal distance of 18 nodes. The collapse of the chaotic dynamics was defined when the mean of the variable $a$, averaged over the whole simulation 


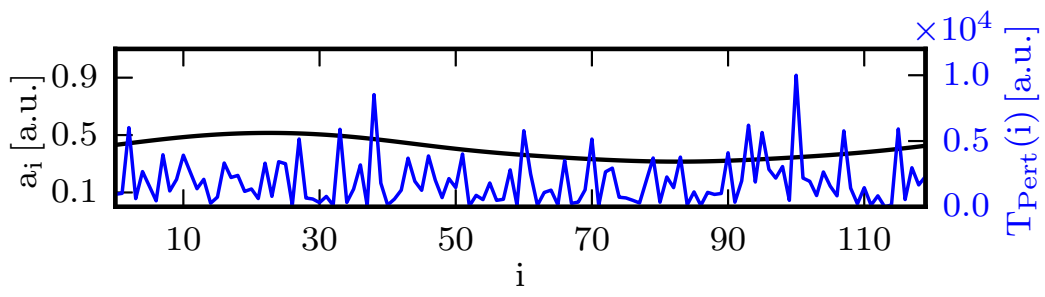

Figure B.4: The impact of the perturbations applied to typical trajectories is shown for the Gray-Scott model. The snapshots of the first variable at the point in time when the perturbations were applied are depicted as the black curve. A perturbation applied at a specific position results in a new trajectory with its own transient lifetime. This lifetime $\mathrm{T}_{\text {Pert }}$ corresponding to the position of the perturbation (of strength $\Delta=0.1$ ) is shown as the blue curve. In general, the transient lifetimes do not seem to show any correlation with the structure of the state at which the perturbations were applied.

domain was above a threshold $\frac{1}{N} \sum_{i} a_{i}>0.99$. For the determination of $\langle\mathrm{T}\rangle_{\mathrm{IC}}$, the amount of time equal to 500 time units was discarded.

\section{Results}

Episodes of spatio-temporal chaos simulated by the Gray-Scott model were investigated using small but finite perturbations. As we found in the other models of the main manuscript, the lifetimes $\mathrm{T}_{\text {Pert }}$ which correspond to perturbed trajectories do not correlate to the actual state (Fig. B.4).

Furthermore, also in the Gray-Scott model we can identify a finite TTP in the final phase, before the self-termination. Figure B.5(a) depicts the analysis of a single trajectory. The magnitude of the perturbation strength has a huge impact on $\langle\mathrm{T}\rangle_{\text {Pert }}$ here.

The chemical concentration $a_{i}$ averaged over all nodes does not provide information about the collapse of the dynamics (lower subplot in Fig. B.5(a)). In order to estimate the length of

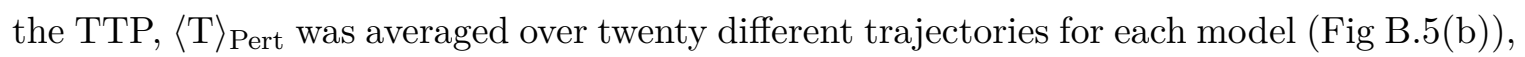
resulting in an approximate $\mathrm{TTP}_{\mathrm{GS}} \approx 60$ a.u., which corresponds to approximately 10 periods of the characterizing patterns of the chaotic dynamic (additionally depicted in Fig. B.3 as the white bar in order to illustrate the length of the TTP corresponding to the actual dynamics).

The underlying dynamics of the TTP can also be detected in the state space. Figure B.6 shows snapshots of the first variable (black curves in (a), (b) and (c)) at three points in time $\left(t_{1}, t_{2}\right.$ and $\left.t_{3}\right)$ which correspond to the vertical black lines in Fig. B.5(a). In addition, the lifetimes of the perturbed trajectories $\mathrm{T}_{\text {Pert }}$ are shown in blue. Comparably to the previous results, clusters of nodes are visible, where perturbations can not prevent the collapse anymore. These clusters grow in time $\left(t_{1}<t_{2}<t_{3}\right)$ until only perturbations at specific nodes can prevent the system from the close collapse. 

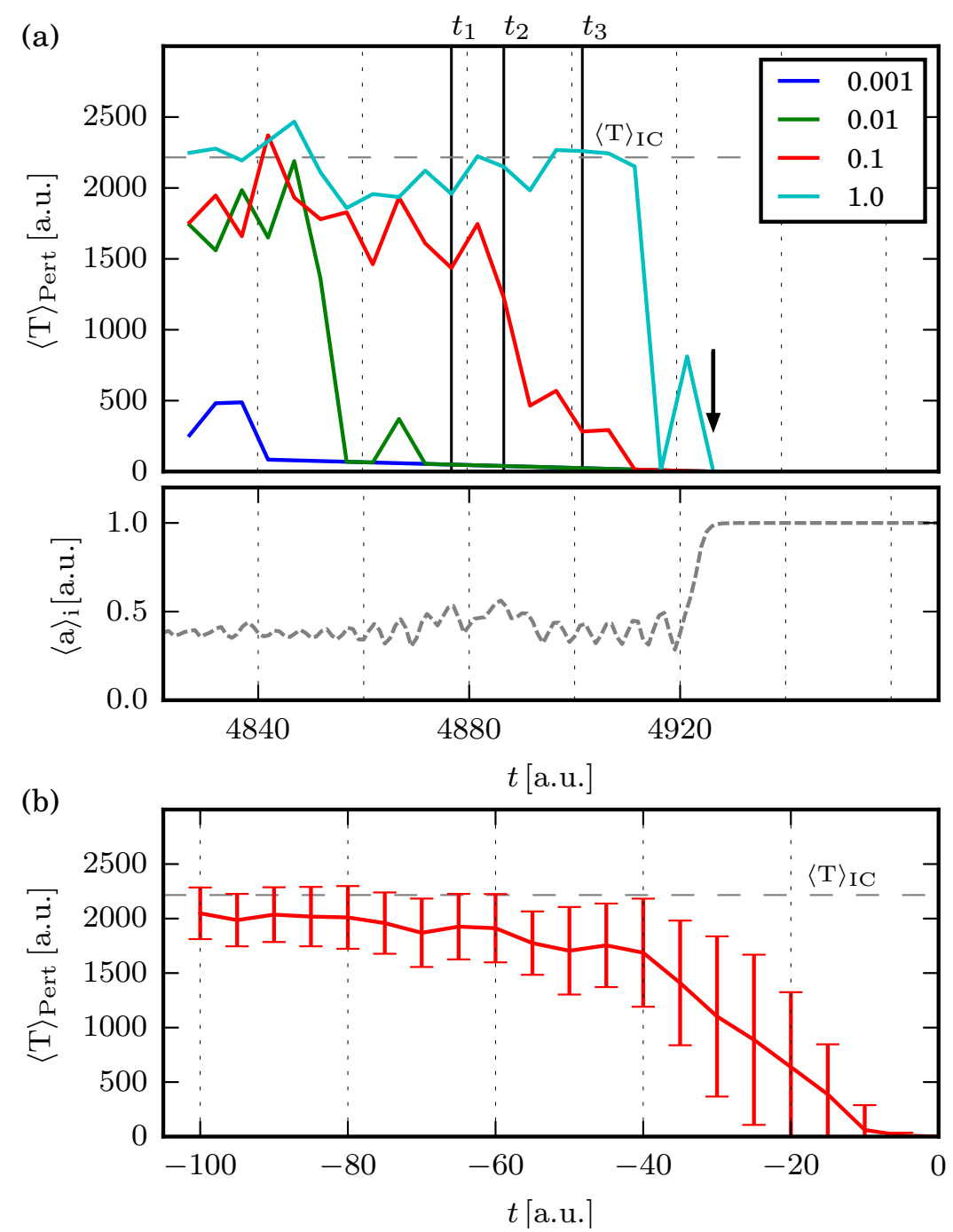

Figure B.5: Temporal correlation of $\langle\mathrm{T}\rangle_{\text {Pert }}$ and the self-termination for the Gray-Scott model. In subplot (a), a single trajectory was chosen, which terminates at around $t=4927$ a.u. (black arrow). $\langle\mathrm{T}\rangle_{\text {Pert }}$ is plotted for various perturbation strengths (in color: $[0.001$, $0.01,0.1,1.00])$. Varying perturbation strengths seem to have a huge impact on $\langle\mathrm{T}\rangle_{\text {Pert }}$. The upcoming collapse is not visible in quantities like the mean (averaged over all nodes) of the variable $a$ (lower subplot of (a)). For a perturbation strength of $\Delta=0.1,\langle\mathrm{~T}\rangle_{\text {Pert }}$ was averaged over twenty trajectories ((b), time axis is normalized such that self-termination occurs at $t=0)$, indicating a $\mathrm{TTP}_{\mathrm{GS}} \approx 60$ a.u. .

\section{B.2.5 Tent Map}

\section{Creation of Chaos and Detection of Termination}

The initial conditions were created by a homogeneous randomized distribution of values between 0 and 1 . Termination was defined, when $x$ exceeded a critical value of $x_{c}=1.0$. 
(a)
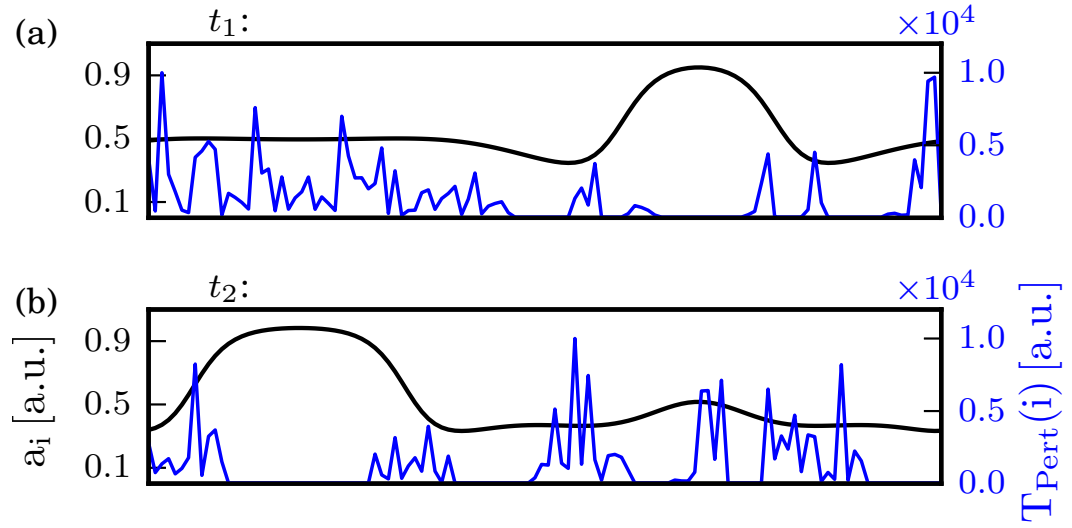

(c)

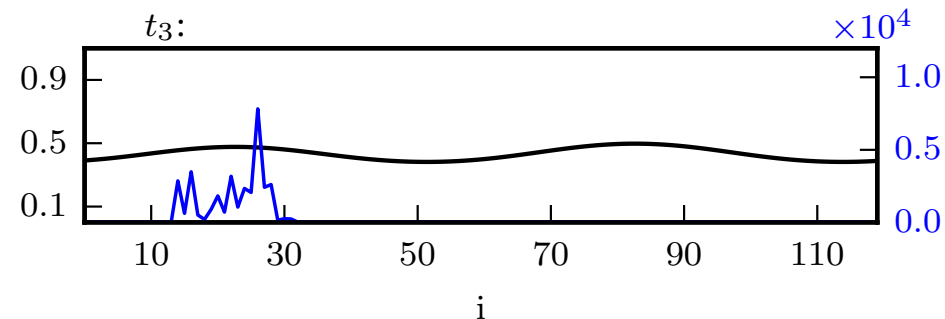

Figure B.6: Snapshots of the first variable of the Gray-Scott model (black) and the lifetimes $\mathrm{T}_{\text {Pert }}(\mathrm{i})$ of trajectories which resulted of a perturbation at node $i$ applied at the time the snapshot was taken (blue) concerning the exemplary trajectory discussed in Fig. B.5(a). The subplots (a), (b) and (c) correspond to three points in time $t_{1}, t_{2}$ and $t_{3}$, respectively, which are also marked by vertical black lines in Fig. B.5(a). Regions where perturbations can not change the average lifetime significantly (with respect to the original, unperturbed trajectory) grow in time.

\section{B.2.6 Hénon Map}

\section{Creation of Chaos and Detection of Termination}

The initial conditions $\left(x_{0}, y_{0}\right)$ were created by a homogeneous randomized distribution on the two-dimensional domain $-3<x<3$ and $-3<y<3$. As stated in the main text, trajectories which leave the chaotic regime run through specific regions in the state space. After passing a small domain around $(x, y) \approx(-1.91,1.8)$ (Fig. 3.16.(d)(iii)), the trajectories diverge to negative infinity (Fig. 3.16(d)(iv)). Thus, the range in time when trajectories terminated could in a first step be identified, when they pass the mentioned regions in state space. The exact definition of the point of termination was then chosen as the beginning of the divergence to negative infinity (one step after passing $(x, y) \approx(-1.91,1.8)$ ). 


\section{B.3 Supplemental Material 3: Complexity Fluctuations dur- ing Ventricular Fibrillation}

\section{B.3.1 3D Simulations}

There are many different cell models from two up to more than thirty dynamical variables. The latter ones aim at describing the cell dynamics on a more realistic electrophysiological level, but are far more computationally demanding. For the $3 \mathrm{D}$ simulations we use the three-variable simplified model (3V-SIM, also known as Fenton-Karma model) [26] since this model already provides the dominant features which are relevant for our scientific objectives like wave break-up and spiral wave meandering which lead to creation and annihilation of spiral or scroll waves.

\section{Fenton-Karma Model}

These $\mathrm{PDE}$ (partial differential equations) were solved using an explicit Euler scheme on a regular grid with the grid size $\left(N_{x}, N_{y}, N_{z}\right)=(151,165,130)$. A spacing of $h_{x}=h_{y}=$ $h_{z}=2.0$ and $d t=0.1$ were used. In general the diffusion constant $\underline{\mathbf{D}}$ in Eq. 3.10 is a spatially dependent second-order tensor since conductivity depends on the local fiber direction. However, we used a homogeneous and isotropic diffusion, thus $\underline{\mathbf{D}}=\mathrm{D}=0.9$. The diffusive part of the PDEs was solved using a 27 point Laplace stencil.

Although the Fenton-Karma model is a relatively simple cell model, different choices of the thirteen parameters of the model already provide a variety of dynamics (e.g. diverse action potentials, wave breakup mechanisms or spiral tip trajectories) [74]. The choice of parameters shows an alternans induced wave breakup far from the tip and a meandering spiral tip (Tab. A.4).

\section{Domain}

It was shown, that the topology of the simulation domain may be essential for the filament dynamics [139]. Length scales like the wave length of a spiral wave or wall thicknesses and their interaction may play an important role concerning the lifetime, production and annihilation rate of filaments. We simulated the electrical wave propagation on a realistic rabbit heart geometry obtained from a CT (Computed Tomography) scan of an animal used in experiments, where only the ventricles were used. Thus, 1.2 million of the 3.2 million voxel of the whole rectangular grid belong effectively to cardiac tissue.

The phase field method yields no-flux boundaries at the irregular boundaries between the cardiac tissue and the surrounding bath or the ventricles[79]. This approach was already used in the same context before 80 .

\section{Protocol}

The sinus rhythm was simulated by giving local stimuli at the apex of the rabbit heart geometry. VF was induced by giving a far field shock in the proper phase of the sinus 


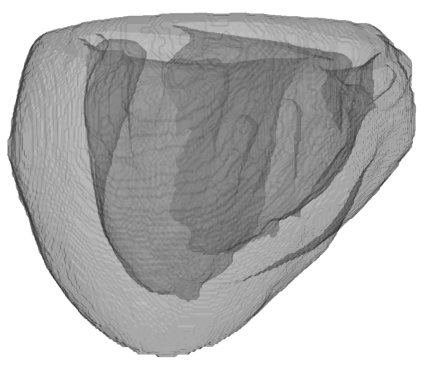

Figure B.7: Exemplary position of the ECG electrode (red star).

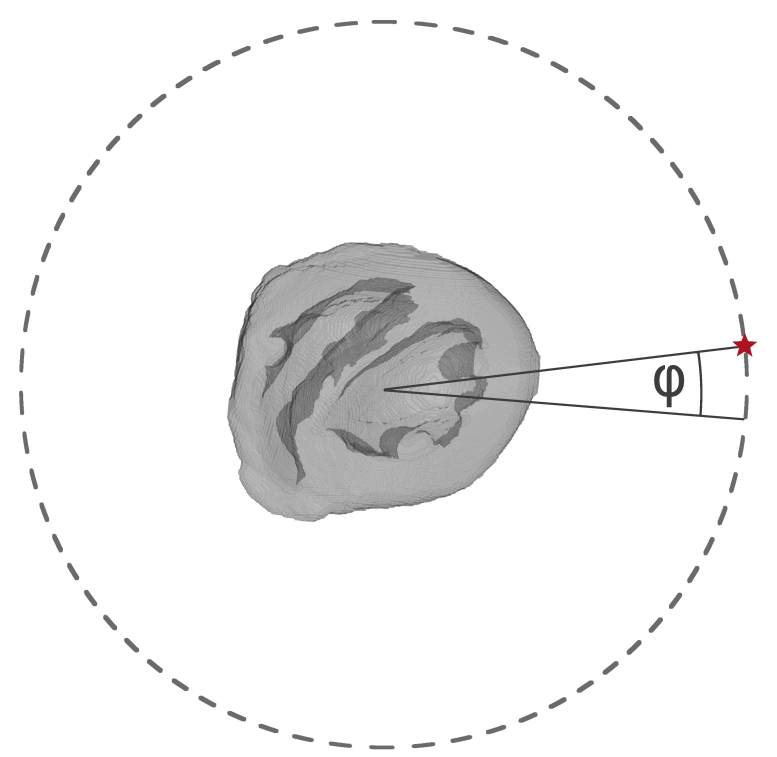

Figure B.8: Exemplary position of the ECG electrode (red star) (view from top on the basis of the heart). The offset of the angle $\varphi$ was chosen arbitrarily.

wave, which leads to symmetry breaking and the creation of scroll waves. Due to different timings of the induction shocks, we created 14 different initial conditions, thus 14 episodes of persistent VF. Local sinus rhythm stimuli were continued during VF.

\section{Electrode Position}

In the third part of the results section, the impact of the electrode position on the measured pseudo ECG was investigated. For this purpose, electrodes were positioned midventricular around $4 \mathrm{~cm}$ away from the epicardium (red star as an exemplary position of the ECG electrode in Fig. B.7). The position of the electrode followed then a circular orbit around the heart (dotted line in Fig. B.8 marks the "orbit" of ECG positions), where the angle $\varphi$ provides the observation angle of the respective electrode.

\section{Filament and Phase Singularity Tracking in Numerical Simulations}

In 3D simulations the organizing centers of the scroll waves, the filaments, where determined via first calculating the pointwise Hilbert transform and subsequent identification of phase singularities by a line integral. Adjacent phase singularities are in a next step identified as 
filaments.

\section{B.3.2 2D Simulations}

\section{D Fenton-Karma}

The two dimensional Fenton-Karma simulations were performed using the same parameters as in 3D (Tab. A.4 and a nine point Laplace stencil. A grid size of $\left(N_{x}, N_{y}\right)=(400,400)$ grid points and a grid spacing of $h_{x}=h_{y}=1.0$ with no-flux boundary conditions have been used. All other parameters were the same as in 3D. A single spiral wave was initialized, which induces a chaotic state by wave breakup. A transient period in the beginning of the simulation has been discarded.

\section{Luo-Rudy Model}

In addition to the Fenton-Karma model, simulations were performed using the phase I of the Luo-Rudy action potential (LR I) model [111]. The aim was to observe and investigate the described phenomena with a more realistic approach from the electrophysiological point of view, keeping still a reasonable level of computational load.

In the LR I model the ionic term introduced in Eq. 3.10 is described by six ionic currents, i.e.

$$
\begin{aligned}
I_{\text {ion }}\left(V_{m}, \mathbf{h}\right)= & -I_{\mathrm{Na}}\left(V_{m}, m, h, j\right)-I_{\mathrm{Ca}}\left(V_{m}, d, f\right) \\
& -I_{\mathrm{K}}\left(V_{m}, X, X_{i}\right)-I_{\mathrm{K} 1}\left(V_{m}\right) \\
& -I_{\mathrm{Kp}}\left(V_{m}\right)-I_{\mathrm{b}}\left(V_{m}\right)
\end{aligned}
$$

where $I_{\mathrm{Na}}$ is the fast sodium current, $I_{\mathrm{si}}$ the slow inward calcium current, $I_{\mathrm{K}}$ the timedependent potassium current, $I_{\mathrm{K} 1}$ the time-independent potassium current, $I_{\mathrm{Kp}}$ the plateau potassium current and $I_{\mathrm{b}}$ the background current. The gating variables $\mathbf{h}$ express the probability for ionic channels to be open or closed depending on membrane voltage $V_{m}$ and are the solutions of the system of nonlinear ordinary differential equations (ODE) introduced by Eq. 3.11. The diffusion tensor, $\underline{\mathbf{D}}$, introduced in Eq. 3.10 is considered to be constant and scalar and set to $0.001 \mathrm{~cm}^{2} / \mathrm{s}$. Other parameters are the same as in the original LR I model. Further details on the equations describing the evolution of the currents and the gating variables are reported in [111]. The simulations were implemented in a $10 \mathrm{~cm} \times 10 \mathrm{~cm}$ sheet of myocardial tissue. No-flux boundary conditions were imposed, with a fixed spacing of $h_{x}=h_{y}=0.025 \mathrm{~cm}$ (resulting in a grid size of $\left(N_{x}, N_{y}\right)=(400,400)$ grid points) and $d t=0.0025 \mathrm{~ms}$ for the diffusive part and $d t=0.01 \mathrm{~ms}$ for the local model. Diffusion was performed as in the 2D Fenton-Karma model using a nine point Laplace stencil.

Since the unmodified ventricular model was used, it was not possible to obtain maintained spiral wave breakup and meandering, as it was already reported in literature for many other simulations using standard models [140, 141, 142, 143, 144, 145]. Therefore, maintenance of reentrant electrical activity was achieved by introducing heterogeneities in the tissue. 
a)

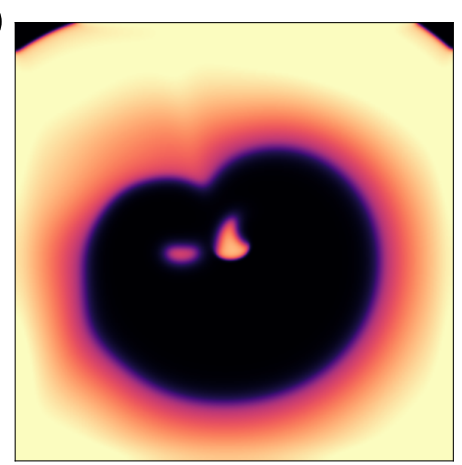

c)

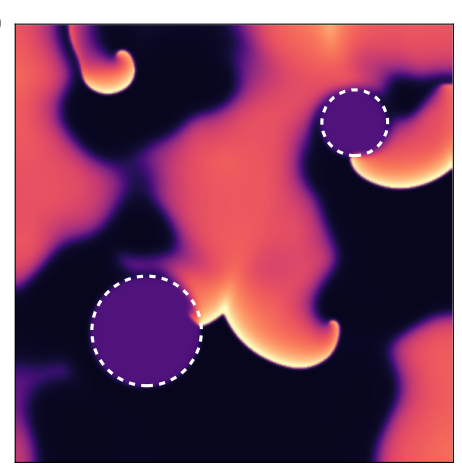

b)

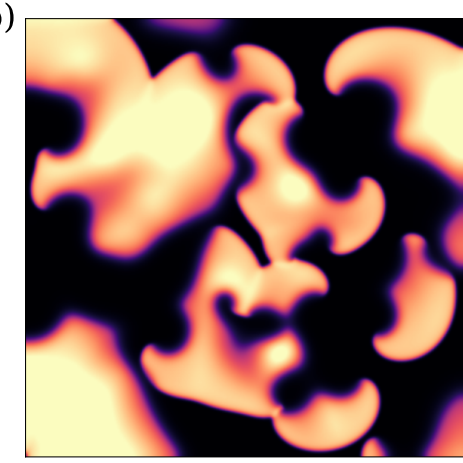

d)

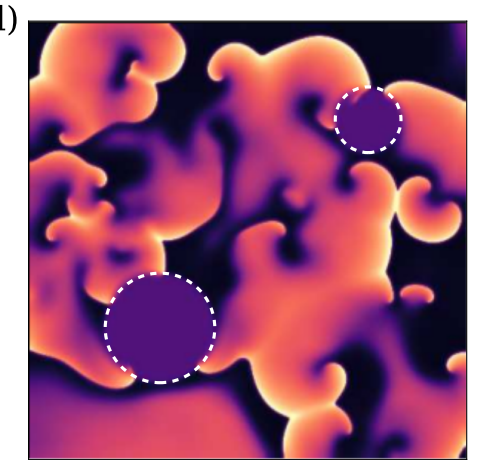

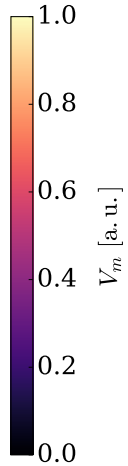

10

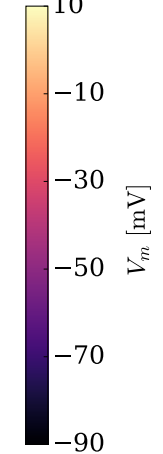

Figure B.9: Snapshots taken from the two dimensional simulations. (a) and (b) show exemplary snapshots of simulations using the Fenton-Karma model : (a) at time step 175 the system is in a less complex state than in (b) at time step 725. (c) and (d) show snapshots from simulations using the Luo-Rudy action potential model: a "laminar" phase (c) at time $2.8 \mathrm{~s}$ and a "complex" phase (d) at time $3.75 \mathrm{~s}$.

In particular, the myocardium was supposed to be affected by acute ischemia and the LR I model was modified accordingly. Ischemic heterogeneities were modelled as regions with reduced conduction properties: conditions of hyperkalemia and acidosis arising at the cellular level were taken into account. In this perspective, the extracellular potassium concentration, $\left[K^{+}\right]_{o}$, was increased from 5.4 to $14 \mathrm{mmol} / \mathrm{L}$ [146]; acidosis exerted its effects on the sodium and L-type calcium channel specific conductances, $g_{\mathrm{Na}}$ and $g_{\mathrm{Ca}}$ (both chosen according to [111]) and were reduced by $25 \%$ [147]. Two ischemic heterogeneities having a radius of $0.75 \mathrm{~cm}$ and $1.25 \mathrm{~cm}$ played the role of obstacles to wave propagation.

The tissue was periodically and locally paced in the left hand row of elements of the sheet. The interval between pulses was held constant and set to $120 \mathrm{~ms}$. Stimuli were current pulses of $2 \mathrm{~ms}$ duration and $300 \mu \mathrm{Acm}^{-2}$ amplitude, delivered for $2 \mathrm{~s}$. Afterwards, the dynamics of the system evolved without any further stimulation until the end of the simulation $(12 \mathrm{~s})$.

\section{Camera and ECG}

For the third part of the results section, two four-electrode custom-built ECGs were used to obtain position dependent ECG time series. In this ex-vivo setup for rabbit hearts, the eight electrodes were arranged approximately equidistant and midventricular around the heart. The four electrodes of each ECG are used to build a Wilson's central terminal which is then 


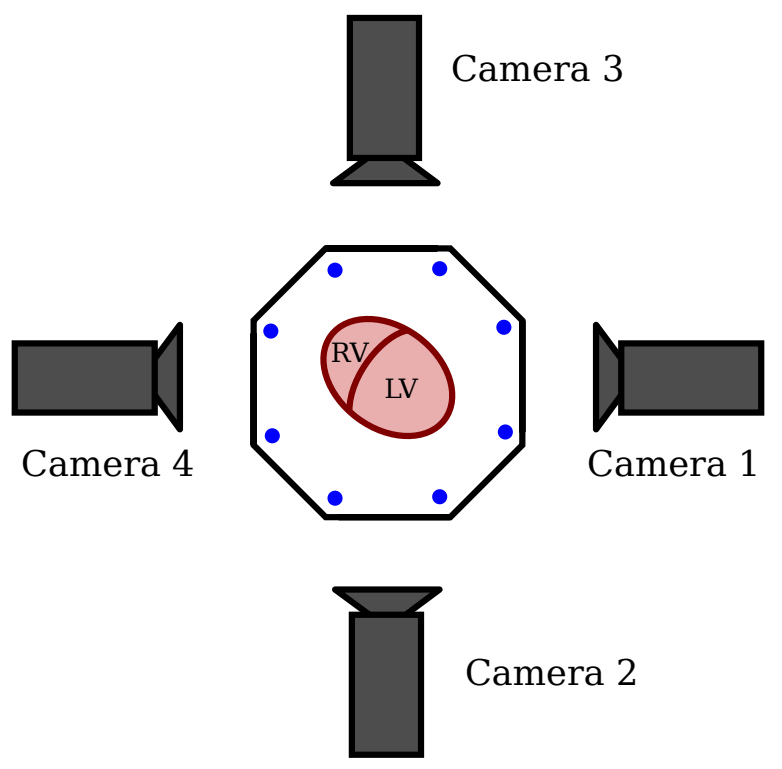

Figure B.10: This sketch shows the experimental setup of ex-vivo experiments. The four cameras used to obtain to obtain the excitation patterns on the surface (optical mapping) are shown, in addition to the position of the eight ECG electrodes (blue dots). "RV" and "LV" denote the right and left ventricle of the heart, respectively.

used as a reference. In the last part the optical mapping approach was used to obtain the electrical excitation patterns on the surface of the heart. For this purpose four cameras where used. Figure B.10 shows the arrangement of the eight ECG electrodes (blue dots) and the four cameras.

\section{B.3.3 Complexity Measures}

The complexity of the system (both in experiments and numerical simulations) was investigated in two ways: the complexity of the ECG was quantified by using the permutation entropy, whereas the spatio-temporal dynamical state (of data from numerical simulations and experimental optical mapping) was analyzed by determining the phase singularities or the filaments of the system, respectively. Details about the calculation procedure can be found in this section.

\section{Permutation Entropy}

Starting from a time series $x_{1}, x_{2}, \ldots, x_{N}$ of length $N$ a series of words of length $D$ is extracted: $w_{i}=\left\{x_{i}, x_{i+1}, \ldots, x_{i+D-1}\right\}=\left\{w_{i, 1}, w_{i, 2} \ldots\right\}$. A symbol number is then assigned to each word which uniquely identifies the relative ordering of its constituting values. The symbol number is computed using the permutation index which is based on a number system with factorial base: 


$$
\begin{aligned}
\hat{w}_{i} & =\sum_{j=1}^{D-1}(D-j) ! \lambda_{j} \\
\lambda_{j} & =\sum_{k=j+1}^{D} \begin{cases}1 & \text { if } w_{j}<w_{k} \\
0 & \text { else }\end{cases}
\end{aligned}
$$

This leads to a series of integer numbers $\hat{w}_{i} \in\left\{0 \leq \kappa_{j} \leq D !-1\right\}$. From this series the relative frequencies $p\left(\kappa_{j}\right)$ of the order pattern can be computed which are then used in the calculation of the permutation entropy:

$$
H=-\sum_{i=0}^{D !-1} p\left(\kappa_{i}\right) \log _{2} p\left(\kappa_{j}\right)
$$

In order to be able to operate on different timescales we use a time delay $L$ for the construction of the words which leads to the slightly modified formula: $w_{i}=\left\{x_{i}, x_{i+L}, \ldots, x_{i+L(D-1)}\right\}$. The permutation indices are then calculated accordingly.

For our analysis we use a windowed version of the PE (window size of $0.5 \mathrm{~s}$ in experiments and 250 frames in numerical simulations). That means that instead of calculating the PE once for the whole length of the signal, we use successive overlapping windows of length $M<N$, calculate the PE $H_{m}$ as described above for each window $m$ and create a time series out of $H_{m}$ values which is displayed in the results section. For the calculation, a pattern length of 4 frames and a lag of $4 \mathrm{~ms}$ has been used. These parameters have been selected based on previous investigations [148].

\section{Phase Synchronization Index}

The phase synchronization index (also called Phase Locking Index [149]) is used to measure the level of synchronization of the electrical patterns on the surface of the heart. For this purpose, the signal (obtained from the optical mapping technique) was extracted at several points on the surface of the heart (see Fig. B.11).

The raw camera signal at these points is filtered using a bandpass filter $(0.2 \mathrm{~Hz}-30 \mathrm{~Hz})$, Gaussian smoothing and a point wise normalization. The phase $\theta_{i}$ of the extracted time series (at point $i$ ) is then determined by detecting action potential upstrokes and interpolating between them from 0 to $2 \pi$. The phase synchronization index between two points based on a time window with the window length $\mathrm{L}_{\text {Win }}=0.5 \mathrm{~s}$ (same window size as used for the calculation of the PE) is then given by Eq. B.13, where the phase difference is averaged over the whole time window. The final phase synchronization index is averaged over all pairs (Eq. B.14). 


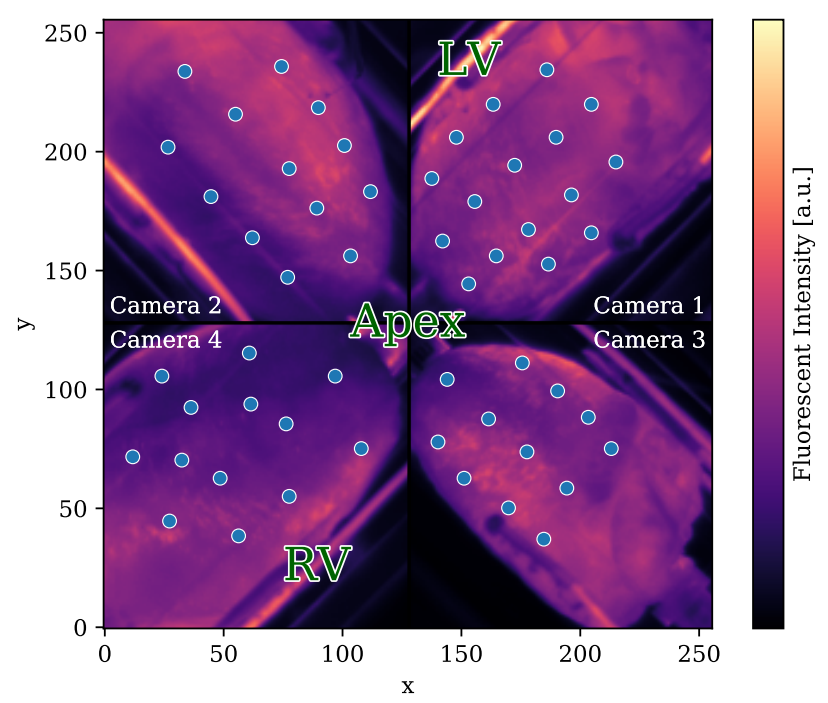

Figure B.11: The distribution of points, used for the calculation of the Phase Synchronization Index. The four parts of the plot show the calculated phase distribution (color coded) based on the data from optical mapping obtained by the four cameras. Blue dots indicate the locations, from where the calculated phase was taken into account for the determination of the PSI.

$$
\begin{aligned}
\operatorname{PSI}_{i, j} & =\left|\frac{1}{\mathrm{~L}_{\mathrm{Win}}}\left\langle e^{i\left(\theta_{i}(t)-\theta_{j}(t)\right)}\right\rangle_{\mathrm{Win}}\right|, \\
\mathrm{PSI} & =\left\langle\mathrm{PSI}_{i, j}\right\rangle_{\text {pairs }} .
\end{aligned}
$$

\section{B.3.4 Results of ECG Fluctuations in Human and Pig hearts}

In order to demonstrate that complexity fluctuations of the ECG during VF is not a phenomenon specific to the species of rabbits, we show in Figs. B.12 and B.13, respectively, excerpts of human (taken from the sudden cardiac death holter database from Physionet [150, 151]) and pig VF episodes along with its permutation entropy. The window size is the same as in the examples for the VF episode of rabbit. Due to a lower sampling rate of $250 \mathrm{~Hz}$ for the human ECG time series instead of $500 \mathrm{~Hz}$ for the animal experiments, some binning artifacts become visible in Fig. B.12, but the difference between the low complexity and high complexity periods are clearly visible in both examples.

\section{Human ECG}

\section{Pig Experiments ECG}

The heart of a Göttingen minipig $(48.6 \mathrm{~kg}$ ) was used. An equivalent sacrifice and heart extraction procedure was used as for the rabbit heart (500 IU kg) heparin, deep anaesthesia with pentobarbital, $1 \mathrm{mmol} / \mathrm{kg} \mathrm{KCl}$ and $400 \mathrm{ml}$ high-potassium cardioplegia solution). 
a)

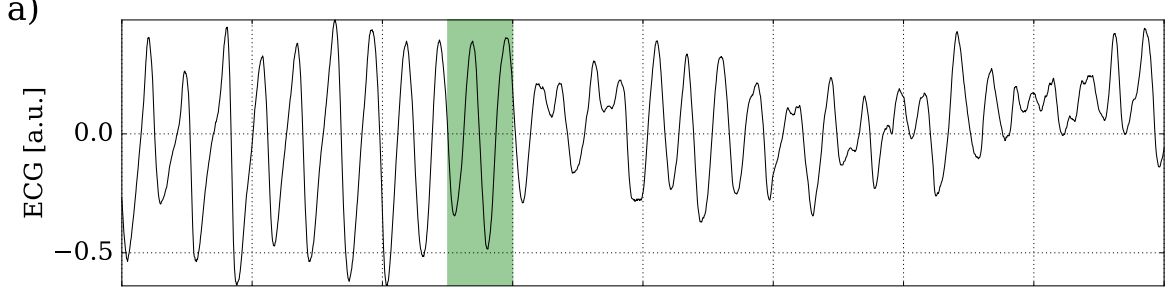

b)

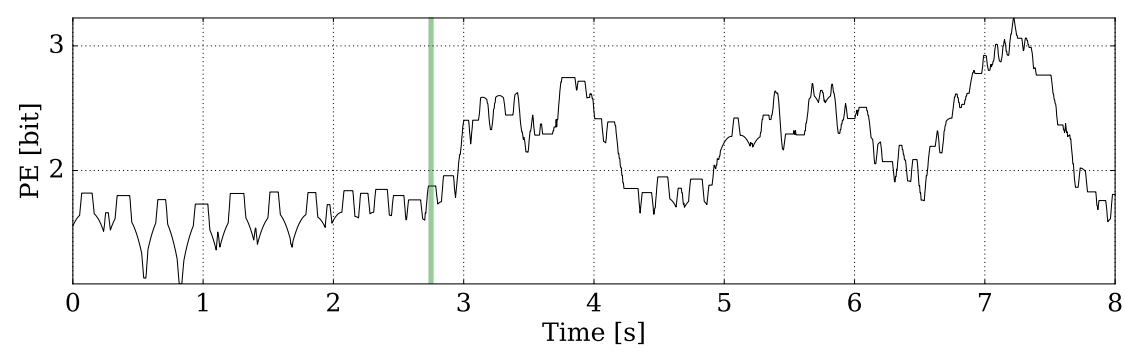

Figure B.12: ECG (a) and PE (b) for a VF episode of human (ECG taken from Physionet [151]). The shaded rectangle visualizes an exemplary time window from which the corresponding PE is calculated (green line in (b)).

A myocardial infarction (MI) [152] was induced in the pig by an occlusion of the second branch of the left anterior descending artery (LAD) as described previously [153] approximately one hour before euthanasia. Figure B.13 shows, that the phenomenon of complexity fluctuations in the ECG also occurs in diseased hearts with MI.

Figure B.13 shows a typical ECG time series (a) and the corresponding PE (b).

\section{B.3.5 Results 2D Simulations}

In addition to the three dimensional simulations in a realistic geometry, simulations on two dimensional rectangular grids have been performed. The local cell dynamics was modeled by the Fenton-Karma and the Luo-Rudy model [111]. The number of phase singularities NPS was used as a measure for the spatio-temporal complexity. We determined the pseudo $\mathrm{ECG}_{\mathrm{Vol}}$ by the mean value of the overall excitation of the cells (Eq. 2.76). As in the three dimensional case, PE was calculated based on this pseudo ECG.

\section{Fenton-Karma Model}

In two dimensional simulations using the Fenton-Karma model, the volumetric ECG $\mathrm{V}_{\mathrm{Vol}}$ again exhibits regular and irregular phases, which are quantified using PE (Fig. B.14a and b). Additionally, the number of phase singularities NPS changed during the episode (Fig. B.14 ). Exemplary snapshots for a "laminar" (time step: 175) and a "complex" phase (time step: 725) are given in Fig. B.9a and b, respectively. During this episode the cross correlation between PE and NPS was $C=0.57$ (both plotted in Fig. B.14b).

\section{Luo-Rudy Model}

In order to show that the discussed correlation between fluctuations in the spatio-temporal state and the PE applied to the ECG is also visible in more sophisticated ionic cell models, 
a)

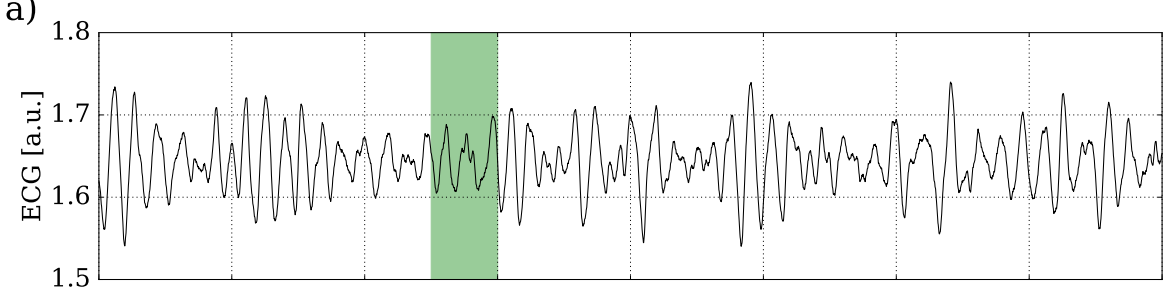

b) 3.5

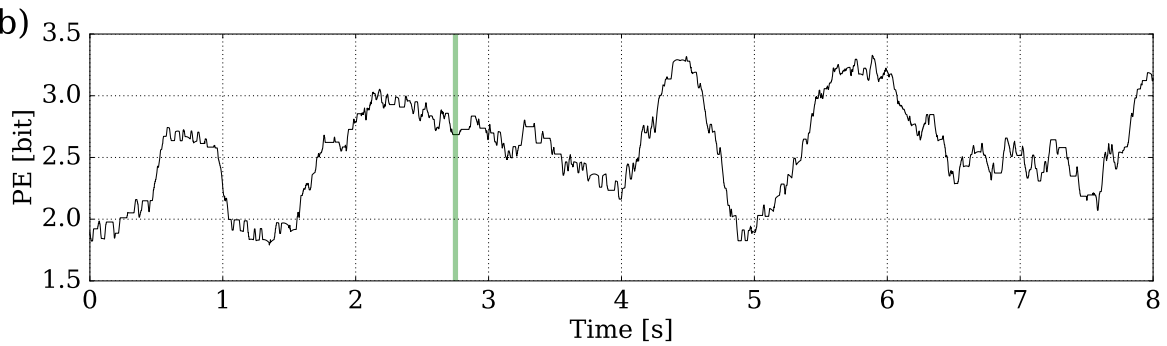

Figure B.13: ECG (see Fig. 3.19) recorded by the attached ECG electrode (see Fig. 3.19) (a) and PE (b) for a VF episode of a pig. The shaded rectangle visualizes an exemplary time window from which the corresponding $\mathrm{PE}$ is calculated (green line in (b)).

two dimensional simulations were performed using the Luo-Rudy model [111]. Figure B.15

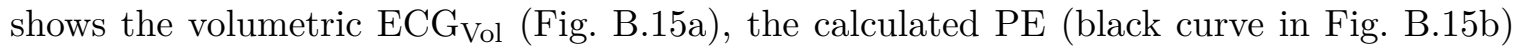
and the number of phase singularities (black curve in Fig. B.15 ). The smoothed version of NPS (red curve in Fig. B.15k) was then replotted in Fig. B.15b as the red dotted line. The cross correlation between PE and NPS in this episode is $C=0.36$.

This analysis shows, that also in two dimensional simulations, the spatio-temporal complexity is visible in the ECG like signal and can be revealed by applying PE.

One interesting fact in this case is, that although the laminar phase can be identified in the $\mathrm{PE}$ time series in (b), it is very difficult to see it in the $\mathrm{ECG}_{\mathrm{Vol}}$ in (a) based on amplitude fluctuations. This is a hint that PE might be a more robust measure for quantifying ECG complexity fluctuations than mere amplitude fluctuations. 


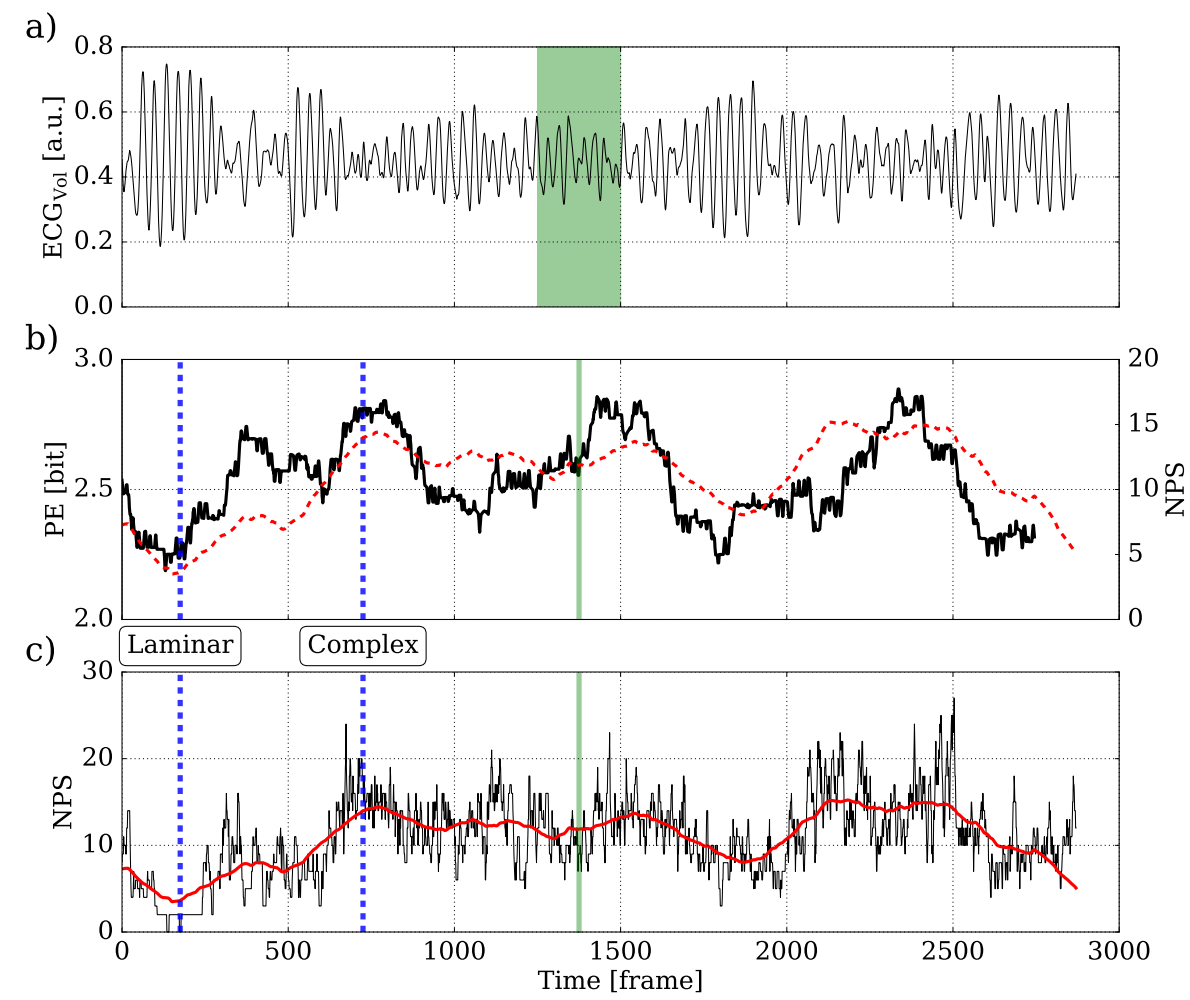

Figure B.14: Investigation of the two dimensional simulation using the Fenton-Karma model. Snapshots of the excitation pattern of a "laminar" phase at time step 175 and a "complex" phase at time step 725 (both marked by blue dotted lines in (b) and (c)) are shown in Fig $\mathrm{B.9}$ ) and b), respectively. The $\mathrm{ECG}_{\mathrm{Vol}}$ and $\mathrm{PE}$ are plotted in (a) and (b) (black curve), respectively. In addition, the number of phase singularities NPS was determined and plotted in (c) (black line). The smoothed signal of NPS (red curve in (c)) is replotted in (b)(red dotted line). 
a)

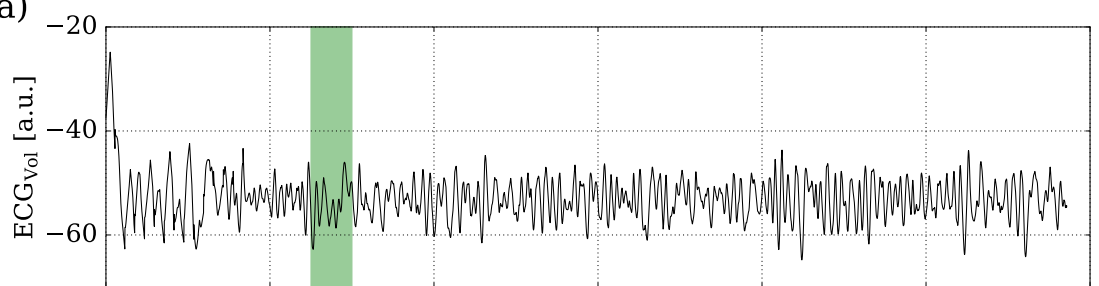

b)

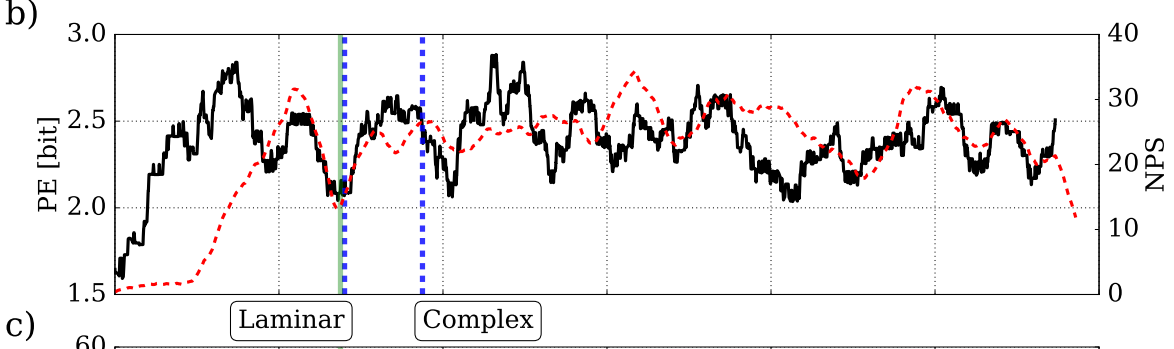

C)

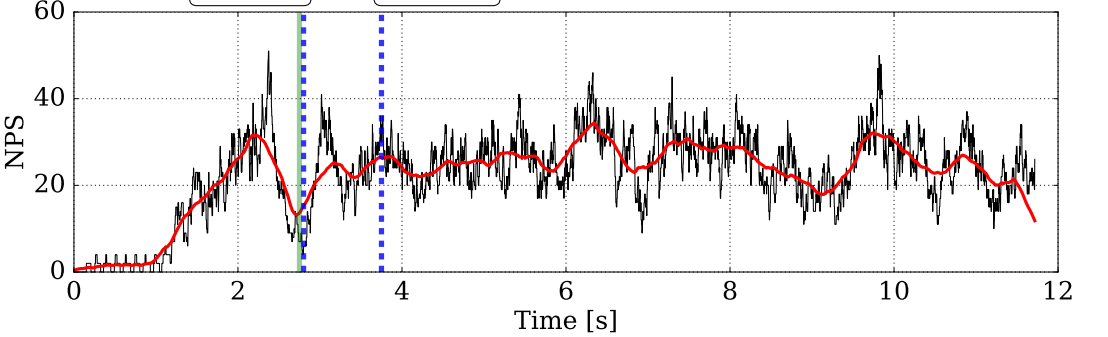

Figure B.15: This figure shows the investigation of a two dimensional simulation using the Luo-Rudy model. Snapshots of the excitation pattern of a "laminar" phase at $2.8 \mathrm{~s}$ and a "complex" phase at $3.75 \mathrm{~s}$ (both marked by blue dotted lines in (b) and (c)) are shown in Fig B.9c) and d), respectively. The ECG $\mathrm{Vol}_{\text {al }}$ and PE is plotted in (a) and (b) (black curve), respectively. In addition, the number of phase singularities NPS was determined and plotted in (c) (black line). The smoothed signal of NPS (red curve in (c)) is replotted in (b) as a red dotted line. 


\section{List of Figures}

1.1 The circulation systems in the human body . . . . . . . . . . . . . . . . 2

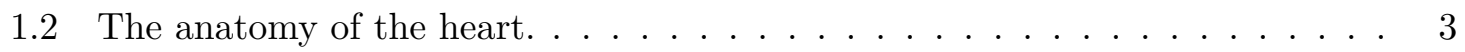

1.3 The contraction of the heart muscle. . . . . . . . . . . . . . . . . . 4

1.4 The difference of the spatio-temporal dynamics between sinus rhythm and ventricular fibrillation and the principle of defibrillation. . . . . . . . . 5

1.5 The transition from sinus rhythm to ventricular fibrillation recorded by an electrocardiogram. . . . . . . . . . . . . . . . . . . . 6

$1.6 \quad$ An implantable cardioverter-defibrillator (ICD). . . . . . . . . . . . . . 7

2.1 The ion leak channels and the sodium-potassium pump. . . . . . . . . . . 12

2.2 The action potential and its underlying mechanism. . . . . . . . . . . . . 14

$2.3 \quad$ A basic description of the mechanism of voltage gated ion channels. $\ldots . . . \quad 15$

2.4 The anatomy of cardiac muscle fibers. . . . . . . . . . . . . . 17

2.5 The arrangement of cardiac fibers in the heart. . . . . . . . . . . . . . 18

2.6 The conduction system of the heart. . . . . . . . . . . . . . . . . . 19

2.7 The structure of a moyofibril $\ldots \ldots \ldots \ldots \ldots \ldots$

2.8 Schematic representation of a usual ECG during sinus rhythm. . . . . . . . . 21

$2.9 \quad$ Recruiting of excitation sites in cardiac tissue dependent on the field strength. 23

2.10 The dose response curve. . . . . . . . . . . . . . . . . . . . 24

2.11 A sketch of a trajectory in state space. . . . . . . . . . . . . 26

2.12 The sinus rhythm in state space. . . . . . . . . . . . . . . . 27

2.13 A saddle point in the state space. . . . . . . . . . . . . . . . 29

2.14 The intersection of the stable and the unstable manifold of a fixed point. . . . 29

2.15 Sustained chaotic dynamics of the logistic map. . . . . . . . . . . . . . 31

2.16 Transient chaotic dynamics of the logistic map. . . . . . . . . . . . . . 32

2.17 Perturbation of a trajectory in state space. . . . . . . . . . . . . . 35

2.18 Lyapunov vectors in tangent space. . . . . . . . . . . . . . . . . . 38

2.19 Pablo Picasso: Der Stier, Zustand I-XI, Lithographien, 1945-46. (c) Succession Picasso/VG Bild-Kunst, Bonn 2018, illustrating the process of modeling different levels of detail. . . . . . . . . . . . . . . . . . . 42

2.20 A representative action potential using the Aliev-Panfilov model. . . . . . . . 50

2.21 A representative action potential using the Fenton-Karma model. . . . . . . . 51

2.22 Discretization grids in different dimensions. . . . . . . . . . . . . . 54 
2.23 The use of "ghost points" in the context of the numerical realization of no-flux

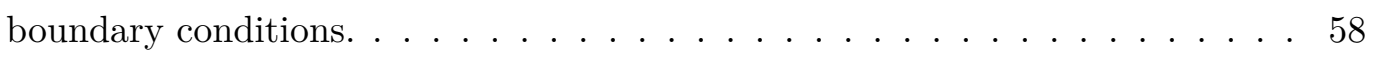

2.24 The Phase Field method. . . . . . . . . . . . . . . . . . . . . . . . . . 59

2.25 Determination of phase singularities using the Hilbert transformation. . . . . 61

2.26 Reduction of a scroll wave to its filament. . . . . . . . . . . . . . . . 62

2.27 Example of a pseudo ECG based on data obtained from numerical simulations. 66

3.1 The (schematic) state space, formulating the scientific objective concerning

\begin{tabular}{|c|}
\hline the study "Features of Chaotic Transients in Excitable Media Governed by \\
\hline
\end{tabular}

Spiral and Scroll Waves". . . . . . . . . . . . . . . . . . . . . 69

3.2 The (schematic) state space, depicting the conclusion of the study "Features of Chaotic Transients in Excitable Media Governed by Spiral and Scroll Waves". 75

3.3 The (schematic) state space, formulating the scientific objective concerning

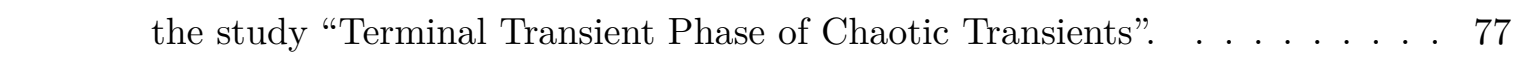

3.4 Spatio-temporal chaos in the Fenton-Karma model, the Bueno-Orovio-Cherry-

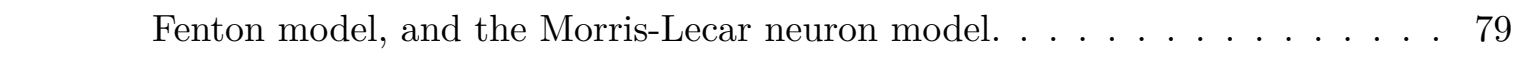

3.5 The number $N_{\mathrm{Ch}}(t)$ of initial conditions which still show chaotic dynamics at time $\mathrm{t}$ is shown over time. . . . . . . . . . . . . . . 83

3.6 Outline of the application of the perturbations, added at specific points in time to the initial trajectory. . . . . . . . . . . . . . . . . 84

3.7 Schematic sketch of the procedure of the application of small perturbations. . 84

3.8 The impact of the perturbations applied to typical trajectories. . . . . . . . 86

3.9 Temporal correlation of $\langle\mathrm{T}\rangle_{\text {Pert }}$ and the self-termination for the Fenton-Karma

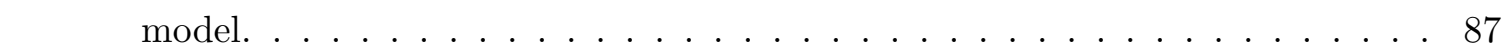

3.10 Temporal correlation of $\langle\mathrm{T}\rangle_{\text {Pert }}$ and the self-termination for the Bueno-Orovio-

Cherry-Fenton model. . . . . . . . . . . . . . . . . 88

3.11 Temporal correlation of $\langle\mathrm{T}\rangle_{\text {Pert }}$ and the self-termination for the Morris-Lecar

3.12 Spatial structure of the lifetime of perturbed trajectories using the FentonKarma model. . . . . . . . . . . . . . . . . . . . . . 92

3.13 Spatial structure of the lifetime of perturbed trajectories using the Bueno-

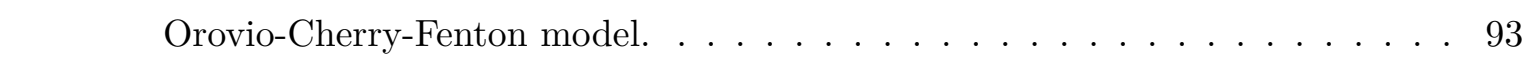

3.14 Snapshots of the first variable of the Morris-Lecar network (black) and the

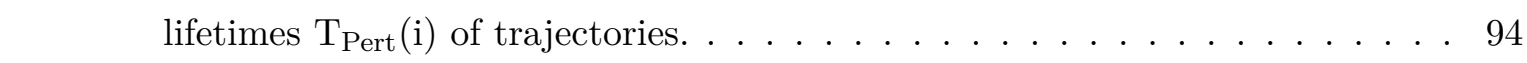

3.15 The Terminal Transient Phase in the tent map. . . . . . . . . . . . . . 96

3.16 The Terminal Transient Phase in the Hénon map. . . . . . . . . . . . . . . . 98

3.17 The (schematic) state space, depicting the conclusion of the study "Terminal -Transient Phase of Chaotic Transients". . . . . . . . . . . . . . . . . . . . . 101

3.18 The (schematic) state space, formulating the scientific objective concerning \begin{tabular}{|c|}
\hline the study "Unveiling Spatio-Temporal Complexity Fluctuations in Cardiac \\
\hline
\end{tabular} Arrhythmia Using Permutation Entropy of ECG Time Series". . . . . . . . . . 103

3.19 A sketch of the experimental setup showing the heart in the perfusion system. 105 
3.20 ECG recorded by the attached ECG electrode and the calculated PE. . . . . 108

3.21 Pseudo ECG and PE computed from a simulated episode of VF using a realistic rabbit heart geometry. . . . . . . . . . . . . . . . 109

3.22 Example of how the dynamics of a scroll wave is reduced to the corresponding filament . . . . . . . . . . . . . . . . . . . . 109

3.23 A comparison of the Permutation Entropy with the number of filaments. . . . 110

3.24 The dependency between the angle of the ECG electrode and the correlation coefficient calculated from NFIL and PE. . . . . . . . . . . . . . . . . 111

3.25 The correlation between NFIL and PEs. . . . . . . . . . . . . . . . . . 112

3.26 Analysis of complexity fluctuations during an episode of VF in a rabbit heart using an eight-electrode setup. . . . . . . . . . . . . . . . . 114

3.27 Comparison of the Real RMSD with the RMSD distribution based on 1000 surrogates. . . . . . . . . . . . . . . . . . . . . 115

3.28 The (schematic) state space, depicting the conclusion of the study "Unveiling Spatio-Temporal Complexity Fluctuations in Cardiac Arrhythmia Using Permutation Entropy of ECG Time Series". . . . . . . . . . . . . . . . . . 116

3.29 The (schematic) state space, formulating the scientific objective concerning the study "Termination Via Perturbation". . . . . . . . . . . . . . . 118

3.30 The principle of the network control problem, shown for a specific example of spatio-temporal chaos in excitable media. . . . . . . . . . . . . 119

3.31 The principle of the network control problem, shown for a specific example

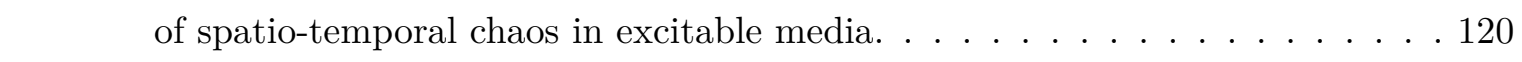

3.32 Selected snapshots of a specific example of spatio-temporal chaos, illustrating the principle of the network control algorithm. . . . . . . . . . . . . 122

3.33 The averaged number of perturbations needed to terminate the dynamics within a given evolution time. . . . . . . . . . . . . 123

B.1 Creation of an exemplary chaotic initial condition of simulations using the - Fenton-Karma model. . . . . . . . . . . . . . . . . . . . 139

B.2 An exemplary episode of self-termination of spatio-temporal chaos governed by spiral waves using the Fenton-Karma model. . . . . . . . . . . . . . . . 140

B.3 A (transient) episode of spatio-temporal chaos using the Gray-Scott model. . 142

B.4 The impact of the perturbations applied to typical trajectories is shown for

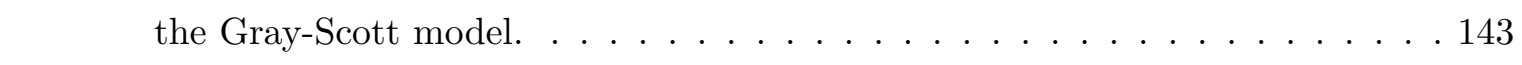

B.5 Temporal correlation of $\langle\mathrm{T}\rangle_{\text {Pert }}$ and the self-termination for the Gray-Scott model. . . . . . . . . . . . . . . . . . . 144

B.6 Snapshots of the first variable of the Gray-Scott model and the lifetimes $\mathrm{T}_{\text {Pert }}(\mathrm{i}) .145$

B.7 Exemplary position of the ECG electrode. . . . . . . . . . . . . . . . 148

B.8 Exemplary position of the ECG electrode. . . . . . . . . . . . . . . . . . 148

B.9 Snapshots taken from the two dimensional simulations. . . . . . . . . . . . 150

B.10 This sketch shows the experimental setup of ex-vivo experiments. . . . . . . . 151 
B.11 The distribution of points, used for the calculation of the Phase Synchronization Index. . . . . . . . . . . . . . . . . . . . . . 153

B.12 ECG and PE for a VF episode of human. . . . . . . . . . . . . . . . . . 154

B.13 ECG and PE for a VF episode of pig. . . . . . . . . . . . . . . . . . . 155

B.14 Investigation of the two dimensional simulation using the Fenton-Karma model.156

B.15 This figure shows the investigation of a two dimensional simulation using the Luo-Rudy model. . . . . . . . . . . . . . . . . . . . . . 157 


\section{List of Tables}

2.1 An overview of experimental measurements of the longitudinal and perpen-

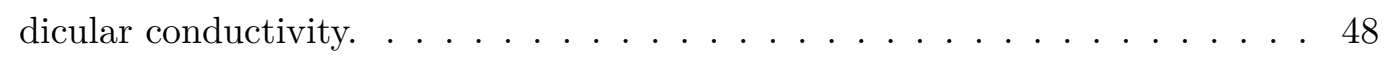

3.1 The average lifetime $\langle\mathrm{T}\rangle_{\text {IC }}$ determined by a fit of the exponential decay of $N_{\mathrm{Ch}}(t)$ for each investigated model. . . . . . . . . . . . . . 82

3.2 The approximate length of the Terminal Transient Phases for each investigated model. . . . . . . . . . . . . . . . . . . . 91

$3.3 \quad$ Saturated correlation $C_{\text {Sat }}$ between NFIL and PE. . . . . . . . . . . . . . 113

A.1 The parameter set AP1, used for simulations with the Aliev-Panfilov model. 131

A.2 The parameter set FK1, used for simulations with the Fenton-Karma model. 132

A.3 The parameter set FK2, used for simulations with the Fenton-Karma model. 132

A.4 The parameter set FK3, used for simulations with the Fenton-Karma model. 132

A.5 The parameter set BOCF1, used for simulations of the Bueno-Orovio-CherryFenton model. . . . . . . . . . . . . . . . . . . . . 134

B.1 The set of parameters used for simulations with the Morris-Lecar network. . . 141

B.2 The set of parameters used for simulations of the Gray-Scott model. . . . . . 142 


\section{Bibliography}

[1] Elaine Nicpon Marieb and Katja Hoehn. Human Anatomy \& Physiology. Pearson, San Francisco, 8th edition, 2010. ISBN 978-0-8053-9569-3 978-0-321-54312-7 978-013-136352-6.

[2] User: Wapcaplet. Diagram of the human heart, created by Wapcaplet in Sodipodi., November 2017. URL https://commons.wikimedia.org/wiki/File:Diagram_of_ the_human_heart_(cropped).svg. Permission is granted to copy, distribute and/or modify this document under the terms of the GNU Free Documentation License, Version 1.2 or any later version published by the Free Software Foundation.

[3] Hong-Wei Tan, Xin-Hua Wang, Hai-Feng Shi, Li Zhou, Jia-Ning Gu, and Xu Liu. Left atrial wall thickness: anatomic aspects relevant to catheter ablation of atrial fibrillation. Chinese Medical Journal, 125(1):12-15, January 2012. ISSN 0366-6999.

[4] Nadine Kawel, Evrim B. Turkbey, J. Jeffrey Carr, John Eng, Antoinette S. Gomes, W. Gregory Hundley, Craig Johnson, Sofia C. Masri, Martin R. Prince, Rob J. van der Geest, João A. C. Lima, and David A. Bluemke. Normal left ventricular myocardial thickness for middle aged and older subjects with SSFP cardiac MR: The MultiEthnic Study of Atherosclerosis. Circulation. Cardiovascular imaging, 5(4):500-508, July 2012. ISSN 1941-9651. doi: 10.1161/CIRCIMAGING.112.973560. URL https: //www.ncbi.nlm.nih.gov/pmc/articles/PMC3412148/

[5] Kelly A. Young, James A. Wise, Peter DeSaix, Dean H. Kruse, Brandon Poe, Eddie Johnson, Jody E. Johnson, Oksana Korol, J. Gordon Betts, and Mark Womble. Anatomy \& Physiology. OpenStax College, 1st edition edition, January 2013. ISBN 978-1-938168-13-0. URL https://opentextbc.ca/anatomyandphysiology/. Published under CC BY-SA 3.0: https://creativecommons.org/licenses/bysa/3.0/deed.en.

[6] Clive M. Baumgarten. Origin of Mechanotransduction: Stretch-Activated Ion Channels. Landes Bioscience, 2013. URL https://www.ncbi.nlm.nih.gov/books/ NBK6374/.

[7] Paul A Iaizzo. Handbook of Cardiac Anatomy, Physiology, and Devices. Springer International Publishing, 2015. ISBN 978-3-319-19464-6 978-3-319-19463-9. URL http://dx.doi.org/10.1007/978-3-319-19464-6. OCLC: 932167659. 
[8] Douglas L. Mann, Douglas P. Zipes, Peter Libby, Robert O. Bonow, and Eugene Braunwald, editors. Braunwald's heart disease: a textbook of cardiovascular medicine. Elsevier/Saunders, Philadelphia, PA, tenth edition edition, 2015. ISBN 978-1-45575134-1 978-1-4557-5133-4 978-99960-963-3-4 978-99960-963-9-6 978-0-323-29429-4.

[9] Aksana Baldzizhar, Ekaterina Manuylova, Roman Marchenko, Yury Kryvalap, and Mary G. Carey. Ventricular Tachycardias: Characteristics and Management. Critical Care Nursing Clinics of North America, 28(3):317-329, September 2016. ISSN 0899-5885. doi: 10.1016/j.cnc.2016.04.004. URL http://www.sciencedirect.com/ science/article/pii/S0899588516300302.

[10] Sanjiv M. Narayan, Frank Bode, Pamela L. Karasik, and Michael R. Franz. Alternans of atrial action potentials during atrial flutter as a precursor to atrial fibrillation. Circulation, 106(15):1968-1973, October 2002. ISSN 1524-4539.

[11] Alessio Gizzi, Elizabeth M. Cherry, Robert F. Gilmour, Stefan Luther, Simonetta Filippi, and Flavio H. Fenton. Effects of pacing site and stimulation history on alternans dynamics and the development of complex spatiotemporal patterns in cardiac tissue. Frontiers in Physiology, 4:71, 2013. ISSN 1664-042X. doi: 10.3389/fphys.2013.00071.

[12] Johnny M. Gomes, Rodrigo Weber Dos Santos, and Elizabeth M. Cherry. Alternans promotion in cardiac electrophysiology models by delay differential equations. Chaos (Woodbury, N.Y.), 27(9):093915, September 2017. ISSN 1089-7682. doi: 10.1063/1. 4999471.

[13] Harold M. Hastings, Flavio H. Fenton, Steven J. Evans, Omer Hotomaroglu, Jagannathan Geetha, Ken Gittelson, John Nilson, and Alan Garfinkel. Alternans and the onset of ventricular fibrillation. Physical Review E, 62(3):4043-4048, September 2000. doi: 10.1103/PhysRevE.62.4043. URL https://link.aps.org/doi/10.1103/ PhysRevE.62.4043.

[14] Scott D Greenwald. Sudden Cardiac Death Holter Database, 1984.

[15] Eugene Braunwald and Robert O. Bonow, editors. Braunwald's heart disease: a textbook of cardiovascular medicine. Saunders, Philadelphia, 9th ed edition, 2012. ISBN 978-1-4377-0398-6 978-1-4377-2708-1 978-0-8089-2436-4.

[16] Medmovie.com. ICD Device, February 2013. URL http://medmovie.com/topic/ cvml_0077a/

[17] Samuel F. Sears, Jessica D. Hauf, Kari Kirian, Garrett Hazelton, and Jamie B. Conti. Posttraumatic Stress and the Implantable Cardioverter-Defibrillator Patient: What the Electrophysiologist Needs to Know. Circulation: Arrhythmia and Electrophysiology, 4(2):242-250, April 2011. ISSN 1941-3149, 1941-3084. doi: 10.1161/CIRCEP. 110.957670. URL http://circep. ahajournals.org/content/4/2/242. 
[18] Gregory P. Walcott, Cheryl R. Killingsworth, and Raymond E. Ideker. Do clinically relevant transthoracic defibrillation energies cause myocardial damage and dysfunction? Resuscitation, 59(1):59-70, October 2003. ISSN 0300-9572.

[19] C. F. Babbs, W. A. Tacker, J. F. VanVleet, J. D. Bourland, and L. A. Geddes. Therapeutic indices for transchest defibrillator shocks: effective, damaging, and lethal electrical doses. American Heart Journal, 99(6):734-738, June 1980. ISSN 0002-8703.

[20] Karl-Heinz Ladwig, Jens Baumert, Birgitt Marten-Mittag, Christof Kolb, Bernhard Zrenner, and Claus Schmitt. Posttraumatic stress symptoms and predicted mortality in patients with implantable cardioverter-defibrillators: results from the prospective living with an implanted cardioverter-defibrillator study. Archives of General Psychiatry, 65(11):1324-1330, November 2008. ISSN 1538-3636. doi: 10.1001/archpsyc.65. 11.1324 .

[21] Stefan Luther, Flavio H. Fenton, Bruce G. Kornreich, Amgad Squires, Philip Bittihn, Daniel Hornung, Markus Zabel, James Flanders, Andrea Gladuli, Luis Campoy, Elizabeth M. Cherry, Gisa Luther, Gerd Hasenfuss, Valentin I. Krinsky, Alain Pumir, Robert F. Gilmour Jr, and Eberhard Bodenschatz. Low-energy control of electrical turbulence in the heart. Nature, 475(7355):235-239, July 2011. ISSN 0028-0836. doi: 10. 1038/nature10216. URL http://www.nature.com/nature/journal/v475/n7355/ full/nature10216.html?foxtrotcallback=true\#supplementary-information.

[22] Ajit H. Janardhan, Sarah R. Gutbrod, Wenwen Li, Di Lang, Richard B. Schuessler, and Igor R. Efimov. Multistage Electrotherapy Delivered Through ChronicallyImplanted Leads Terminates Atrial Fibrillation With Lower Energy Than a Single Biphasic Shock. Journal of the American College of Cardiology, 63(1):40-48, January 2014. ISSN 0735-1097. doi: 10.1016/j.jacc.2013.07.098. URL https: //www.ncbi.nlm.nih.gov/pmc/articles/PMC4123180/

[23] D. E. Goldman. POTENTIAL, IMPEDANCE, AND RECTIFICATION IN MEMBRANES. The Journal of General Physiology, 27(1):37-60, September 1943. ISSN 0022-1295.

[24] John E. Hall and Arthur C. Guyton. Guyton and Hall textbook of medical physiology. Saunders/Elsevier, Philadelphia, Pa, 12th ed edition, 2011. ISBN 978-1-4160-4574-8.

[25] Yoram Rudy. Molecular basis of cardiac action potential repolarization. Annals of the New York Academy of Sciences, 1123:113-118, March 2008. ISSN 0077-8923. doi: 10.1196/annals.1420.013.

[26] Flavio Fenton and Alain Karma. Vortex dynamics in three-dimensional continuous myocardium with fiber rotation: Filament instability and fibrillation. Chaos: An Interdisciplinary Journal of Nonlinear Science, 8(1):20, 1998. ISSN 10541500. doi: 10. 1063/1.166311. URL http://scitation.aip.org/content/aip/journal/chaos/8/ $1 / 10.1063 / 1.166311$. 
[27] Derivative: Hazmat2Original: Hank van Helvete. English: Normal ECG/EKG complex with labels, March 2014. URL https://commons.wikimedia.org/wiki/File: EKG_Complex_en.svg. Permission is granted to copy, distribute and/or modify this document under the terms of the GNU Free Documentation License, Version 1.2 or any later version published by the Free Software Foundation.

[28] Igor Efimov and Crystal Ripplinger. Virtual electrode hypothesis of defibrillation. Heart rhythm : the official journal of the Heart Rhythm Society, 3:1100-2, October 2006. doi: 10.1016/j.hrthm.2006.03.005.

[29] A. Pumir, V. Nikolski, M. Hörning, A. Isomura, K. Agladze, K. Yoshikawa, R. Gilmour, E. Bodenschatz, and V. Krinsky. Wave emission from heterogeneities opens a way to controlling chaos in the heart. Physical Review Letters, 99(20):208101, November 2007. ISSN 0031-9007. doi: 10.1103/PhysRevLett.99.208101.

[30] Natalia Trayanova, Kirill Skouibine, and Felipe Aguel. The role of cardiac tissue structure in defibrillation. Chaos (Woodbury, N.Y.), 8(1):221-233, March 1998. ISSN 1089-7682. doi: 10.1063/1.166299.

[31] Philip Bittihn, Marcel Hörning, and Stefan Luther. Negative Curvature Boundaries as Wave Emitting Sites for the Control of Biological Excitable Media. Physical Review Letters, 109:118106, September 2012. doi: 10.1103/PhysRevLett.109.118106.

[32] Kevin F. Kwaku and Stephen M. Dillon. Shock-Induced Depolarization of Refractory Myocardium Prevents Wave-Front Propagation in Defibrillation. Circulation Research, 79(5):957-973, November 1996. ISSN 0009-7330, 1524-4571. doi: 10.1161/01.RES.79. 5.957. URL http://circres . ahajournals .org/content/79/5/957.

[33] M. Hénon. A two-dimensional mapping with a strange attractor. Communications in Mathematical Physics, 50(1):69-77, February 1976. ISSN 0010-3616, 1432-0916. doi: 10.1007/BF01608556. URL https://link.springer.com/article/10.1007/ BF01608556.

[34] Ian Stewart. Sources of uncertainty in deterministic dynamics: an informal overview. Phil. Trans. R. Soc. A, 369(1956):4705-4729, December 2011. ISSN 1364-503X, 14712962. doi: 10.1098/rsta.2011.0113. URL http://rsta.royalsocietypublishing. org/content/369/1956/4705.

[35] Hans-Walther Lorenz and Helena E. Nusse. Chaotic attractors, chaotic saddles, and fractal basin boundaries: Goodwin's nonlinear accelerator model reconsidered. Chaos Solitons and Fractals, 13:957-965, April 2002. ISSN 0960-0779. doi: 10.1016/S0960-0779(01)00121-7. URL http://adsabs.harvard.edu/abs/2002CSF. $\ldots 13 . .957 \mathrm{~L}$.

[36] Holger Faisst and Bruno Eckhardt. Traveling Waves in Pipe Flow. Physical Review Letters, 91(22):224502, November 2003. doi: 10.1103/PhysRevLett.91.224502. URL https://link.aps.org/doi/10.1103/PhysRevLett.91.224502. 
[37] S. P. Ellner and P. Turchin. When can noise induce chaos and why does it matter: a critique. Oikos, 111(3):620-631, December 2005. ISSN 00301299, 16000706. doi: 10.1111/j.1600-0706.2005.14129.x. URL http://doi.wiley.com/10.1111/j. $1600-0706.2005 .14129 . x$.

[38] Ben Poole, Subhaneil Lahiri, Maithra Raghu, Jascha Sohl-Dickstein, and Surya Ganguli. Exponential expressivity in deep neural networks through transient chaos. arXiv:1606.05340, June 2016.

[39] Xiao-Song Yang and Quan Yuan. Chaos and transient chaos in simple Hopfield neural networks. Neurocomputing, 69(1):232-241, December 2005. ISSN 09252312. doi: 10.1016/j.neucom.2005.06.005. URL http://www.sciencedirect.com/ science/article/pii/S0925231205001773

[40] Xiangbao Wu and Hans Liljenstrom. Regulating the nonlinear dynamics of olfactory cortex. Network: Computation in Neural Systems, 5(1):47-60, January 1994. ISSN 0954-898X. doi: 10.1088/0954-898X_5_1_003. URL http://www.tandfonline.com/ doi/abs/10.1088/0954-898X_5_1_003.

[41] Ying-Cheng Lai and Tamás Tél. Transient Chaos. Applied Mathematical Sciences. Springer, New York, NY, 2011. ISBN 978-1-4419-6986-6 978-1-4419-6987-3. URL http://link.springer.com/10.1007/978-1-4419-6987-3.

[42] Michael Widom, David Bensimon, Leo P. Kadanoff, and Scott J. Shenker. Strange objects in the complex plane. Journal of Statistical Physics, 32(3):443-454, September 1983. ISSN 0022-4715, 1572-9613. doi: 10.1007/BF01008949. URL https://link. springer.com/article/10.1007/BF01008949.

[43] James P. Crutchfield and Kunihiko Kaneko. Are Attractors Relevant to Turbulence? Physical Review Letters, 60(26):2715-2718, June 1988. ISSN 0031-9007. doi: 10.1103/ PhysRevLett.60.2715. URL http://link.aps.org/doi/10.1103/PhysRevLett.60. 2715 .

[44] V.I. Osedelec. A multiplicative ergodic theorem: Lyapunov characteristic exponents for dynamical systems. Trans. Mosc. Math. Soc., 19:197-231, January 1968.

[45] Pavel V. Kuptsov and Ulrich Parlitz. Theory and computation of covariant Lyapunov vectors. Journal of Nonlinear Science, 22(5):727-762, October 2012. ISSN 0938-8974, 1432-1467. doi: 10.1007/s00332-012-9126-5. URL http://arxiv.org/abs/1105. 5228, arXiv: 1105.5228.

[46] Giancarlo Benettin, Luigi Galgani, Antonio Giorgilli, and Jean-Marie Strelcyn. Lyapunov Characteristic Exponents for smooth dynamical systems and for hamiltonian systems; a method for computing all of them. Part 1: Theory. Meccanica, 15(1): 9-20, March 1980. ISSN 0025-6455, 1572-9648. doi: 10.1007/BF02128236. URL https://link.springer.com/article/10.1007/BF02128236. 
[47] James L. Kaplan and James A. Yorke. Chaotic behavior of multidimensional difference equations. In Heinz-Otto Peitgen and Hans-Otto Walther, editors, Functional Differential Equations and Approximation of Fixed Points, volume 730, pages 204227. Springer Berlin Heidelberg, Berlin, Heidelberg, 1979. ISBN 978-3-540-09518-7 978-3-540-35129-0. URL http://link. springer.com/10.1007/BFb0064319.

[48] A. Rényi. On the dimension and entropy of probability distributions. Acta Mathematica Academiae Scientiarum Hungarica, 10(1-2):193-215, March 1959. ISSN 0001-5954, 1588-2632. doi: 10.1007/BF02063299. URL https://link.springer.com/article/ 10.1007/BF02063299.

[49] Lai-Sang Young. Dimension, entropy and Lyapunov exponents. Ergodic Theory and Dynamical Systems, 2(1):109-124, March 1982. ISSN 14694417, 0143-3857. doi: 10.1017/S0143385700009615. URL https://www. cambridge.org/core/journals/ergodic-theory-and-dynamical-systems/ article/dimension-entropy-and-lyapunov-exponents/

5B6962A34BACD4A07EA5C7B6AE539051.

[50] F. Ledrappier and L.-S. Young. Dimension formula for random transformations. Communications in Mathematical Physics, 117(4):529-548, 1988. ISSN 0010-3616, 14320916. URL https://projecteuclid.org/euclid.cmp/1104161815.

[51] Holger Kantz and Thomas Schreiber. Nonlinear Time Series Analysis, November 2003. URL /core/books/nonlinear-time-series-analysis/ 519783E4E8A2C3DCD4641E42765309C7.

[52] H. Kantz and P. Grassberger. Repellers, semi-attractors, and long-lived chaotic transients. Physica D Nonlinear Phenomena, 17:75-86, August 1985. ISSN 0167-2789. doi: 10.1016/0167-2789(85)90135-6. URL http://adsabs.harvard.edu/abs/1985PhyD. $\ldots 17 \ldots 75 \mathrm{~K}$

[53] null Hunt, null Ott, and null Yorke. Fractal dimensions of chaotic saddles of dynamical systems. Physical Review. E, Statistical Physics, Plasmas, Fluids, and Related Interdisciplinary Topics, 54(5):4819-4823, November 1996. ISSN 1063-651X.

[54] Edward N. Lorenz. Deterministic Nonperiodic Flow. Journal of the Atmospheric Sciences, 20(2):130-141, March 1963. ISSN 0022-4928. doi: 10.1175/1520-0469(1963) 020<0130:DNF $\rangle$ 2.0.CO;2. URL http://journals.ametsoc.org/doi/abs/10.1175/ $1520-0469 \% 281963 \% 29020 \% 3 \mathrm{C} 0130 \% 3 \mathrm{ADNF} \% 3 \mathrm{E} 2.0 . \mathrm{CO} \% 3 \mathrm{~B} 2$.

[55] Daniel Hornung. Cardiac Arrhythmia Termination on the Vascular and Organ Scale. $\mathrm{PhD}$ thesis, Georg-August Universitaet Goettingen, November 2014. URL https: //ediss.uni-goettingen.de/handle/11858/00-1735-0000-0023-9934-8.

[56] Pablo Picasso. How to Design Like Apple: The Picasso Way, November 2017. URL http://echopresentations.blogspot.com/2014/08/ how-to-design-like-apple.html. 
[57] Remi Peyronnet, Jeanne M. Nerbonne, and Peter Kohl. Cardiac Mechano-Gated Ion Channels and Arrhythmias. Circulation research, 118(2):311-329, January 2016. ISSN 0009-7330. doi: 10.1161/CIRCRESAHA.115.305043. URL https://www.ncbi.nlm. nih.gov/pmc/articles/PMC4742365/

[58] Henry C. Tuckwell. Introduction to theoretical neurobiology. Number 8 in Cambridge studies in mathematical biology. Cambridge University Press, Cambridge [Cambridgeshire] ; New York, 1988. ISBN 978-0-521-35096-9.

[59] John C. Clements, Jukka Nenonen, P. K. J. Li, and B. Milan Horáček. Activation Dynamics in Anisotropic Cardiac Tissue via Decoupling. Annals of Biomedical Engineering, 32(7):984-990, July 2004. ISSN 0090-6964, 1573-9686. doi: 10. 1023/B:ABME.0000032461.80932.eb. URL https://link.springer.com/article/ 10.1023/B:ABME.0000032461.80932.eb.

[60] L. S. Green, B. Taccardi, P. R. Ershler, and R. L. Lux. Epicardial potential mapping. Effects of conducting media on isopotential and isochrone distributions. Circulation, 84(6):2513-2521, December 1991. ISSN 0009-7322, 1524-4539. doi: 10.1161/01.CIR. 84.6.2513. URL http://circ. ahajournals.org/content/84/6/2513.

[61] Muriel Boulakia, Serge Cazeau, Miguel A. Fernández, Jean-Frédéric Gerbeau, and Nejib Zemzemi. Mathematical modeling of electrocardiograms: a numerical study. Annals of Biomedical Engineering, 38(3):1071-1097, March 2010. ISSN 1573-9686. doi: 10.1007/s10439-009-9873-0.

[62] A. Pumir and V. Krinsky. Unpinning of a rotating wave in cardiac muscle by an electric field. Journal of Theoretical Biology, 199(3):311-319, August 1999. ISSN 0022-5193. doi: 10.1006/jtbi.1999.0957.

[63] Philip Bittihn, Gisela Luther, Eberhard Bodenschatz, Valentin Krinsky, Ulrich Parlitz, and Stefan Luther. Far field pacing supersedes anti-tachycardia pacing in a generic model of excitable media. New Journal of Physics, 10(10):103012, 2008. ISSN 13672630. doi: 10.1088/1367-2630/10/10/103012.

[64] L. Clerc. Directional differences of impulse spread in trabecular muscle from mammalian heart. The Journal of Physiology, 255(2):335-346, February 1976. ISSN 00223751.

[65] D. E. Roberts, L. T. Hersh, and A. M. Scher. Influence of cardiac fiber orientation on wavefront voltage, conduction velocity, and tissue resistivity in the dog. Circulation Research, 44(5):701-712, May 1979. ISSN 0009-7330.

[66] D. E. Roberts and A. M. Scher. Effect of tissue anisotropy on extracellular potential fields in canine myocardium in situ. Circulation Research, 50(3):342-351, March 1982. ISSN 0009-7330. 
[67] Mark Potse, Bruno Dubé, Jacques Richer, Alain Vinet, and Ramesh M. Gulrajani. A comparison of monodomain and bidomain reaction-diffusion models for action potential propagation in the human heart. IEEE transactions on bio-medical engineering, 53(12 Pt 1):2425-2435, December 2006. ISSN 0018-9294. doi: 10.1109/TBME.2006. 880875 .

[68] Yves Bourgault and Charles Pierre. Comparing the bidomain and monodomain models in electro-cardiology through convergence analysis, December 2010. URL https: //hal.archives-ouvertes.fr/hal-00545888/document.

[69] J P Wikswo, S F Lin, and R A Abbas. Virtual electrodes in cardiac tissue: a common mechanism for anodal and cathodal stimulation. Biophysical Journal, 69(6):21952210, December 1995. ISSN 0006-3495. URL http://www.ncbi.nlm.nih.gov/pmc/ articles/PMC1236459/.

[70] John P. Wikswo and Bradley J. Roth. Virtual Electrode Theory of Pacing. In Cardiac Bioelectric Therapy, pages 283-330. Springer, Boston, MA, 2009. ISBN 978-0-38779402-0 978-0-387-79403-7. doi: 10.1007/978-0-387-79403-7_12. URL https://link. springer.com/chapter/10.1007/978-0-387-79403-7_12.

[71] Dwight Barkley. A model for fast computer simulation of waves in excitable media. Physica D: Nonlinear Phenomena, 49(1):61-70, April 1991. ISSN 0167-2789. doi: 10.1016/0167-2789(91)90194-E. URL http://www.sciencedirect.com/science/ article/pii/016727899190194E.

[72] Rubin R. Aliev and Alexander V. Panfilov. A simple two-variable model of cardiac excitation. Chaos, Solitons \& Fractals, 7(3):293-301, March 1996. ISSN 09600779. doi: 10.1016/0960-0779(95)00089-5. URL http://linkinghub.elsevier.com/retrieve/ pii/0960077995000895

[73] C. H. Luo and Y. Rudy. A dynamic model of the cardiac ventricular action potential. I. Simulations of ionic currents and concentration changes. Circulation Research, 74 (6):1071-1096, June 1994. ISSN 0009-7330.

[74] Flavio H. Fenton, Elizabeth M. Cherry, Harold M. Hastings, and Steven J. Evans. Multiple mechanisms of spiral wave breakup in a model of cardiac electrical activity. Chaos: An Interdisciplinary Journal of Nonlinear Science, 12(3):852-892, September 2002. ISSN 1054-1500, 1089-7682. doi: 10.1063/1.1504242. URL http://scitation. aip.org/content/aip/journal/chaos/12/3/10.1063/1.1504242

[75] William H. Press, editor. Numerical recipes: the art of scientific computing. Cambridge University Press, Cambridge, UK ; New York, 3rd ed edition, 2007. ISBN 978-0-52188068-8 978-0-521-88407-5 978-0-521-70685-8. OCLC: ocn123285342.

[76] J. C. Butcher. Numerical methods for ordinary differential equations. J. Wiley, Chichester, West Sussex, England ; Hoboken, NJ, 2003. ISBN 978-0-471-96758-3. 
[77] Philip Bittihn. Complex Structure and Dynamics of the Heart. Springer, December 2014. ISBN 978-3-319-12232-8. Google-Books-ID: 5LTzBQAAQBAJ.

[78] John C. Strikwerda. Finite difference schemes and partial differential equations. Society for Industrial and Applied Mathematics, Philadelphia, 2nd ed edition, 2004. ISBN 978-0-89871-567-5.

[79] X. Li, J. Lowengrub, A. Rätz, and A. Voigt. Solving PDEs in Complex Geometries: a Diffuse Approach. Communications in mathematical sciences, 7(1):81-107, March 2009. ISSN 1539-6746. URL http://www.ncbi.nlm.nih.gov/pmc/articles/ PMC3097555/.

[80] Flavio H. Fenton, Elizabeth M. Cherry, Alain Karma, and Wouter-Jan Rappel. Modeling wave propagation in realistic heart geometries using the phase-field method. Chaos: An Interdisciplinary Journal of Nonlinear Science, 15(1):013502, 2005. ISSN 10541500. doi: 10.1063/1.1840311. URL http://scitation.aip.org/content/aip/ journal/chaos/15/1/10.1063/1.1840311.

[81] R. H. Clayton, E. A. Zhuchkova, and A. V. Panfilov. Phase singularities and filaments: Simplifying complexity in computational models of ventricular fibrillation. Progress in Biophysics and Molecular Biology, 90(1):378-398, January 2006. ISSN 0079-6107. doi: 10.1016/j.pbiomolbio.2005.06.011. URL http://www.sciencedirect.com/science/ article/pii/S0079610705000520

[82] Mark-Anthony Bray and John P. Wikswo. Considerations in phase plane analysis for nonstationary reentrant cardiac behavior. Physical Review. E, Statistical, Nonlinear, and Soft Matter Physics, 65(5 Pt 1):051902, May 2002. ISSN 1539-3755. doi: 10. 1103/PhysRevE.65.051902.

[83] M. A. Bray, S. F. Lin, R. R. Aliev, B. J. Roth, and J. P. Wikswo. Experimental and theoretical analysis of phase singularity dynamics in cardiac tissue. Journal of Cardiovascular Electrophysiology, 12(6):716-722, June 2001. ISSN 1045-3873.

[84] Mark-Anthony Bray and John P. Wikswo. Use of topological charge to determine filament location and dynamics in a numerical model of scroll wave activity. IEEE transactions on bio-medical engineering, 49(10):1086-1093, October 2002. ISSN 00189294. doi: 10.1109/TBME.2002.803516.

[85] null Goryachev and null Kapral. Spiral waves in chaotic systems. Physical Review Letters, 76(10):1619-1622, March 1996. ISSN 1079-7114. doi: 10.1103/PhysRevLett. 76.1619 .

[86] N. D. Mermin. The topological theory of defects in ordered media. Reviews of Modern Physics, 51(3):591-648, July 1979. doi: 10.1103/RevModPhys.51.591. URL https: //link.aps.org/doi/10.1103/RevModPhys.51.591. 
[87] Robert Plonsey and Roger C. Barr. Bioelectricity: a quantitative approach. Springer, New York, NY, 3rd ed edition, 2007. ISBN 978-0-387-48864-6.

[88] Thomas Lilienkamp, Jan Christoph, and Ulrich Parlitz. Features of Chaotic Transients in Excitable Media Governed by Spiral and Scroll Waves. Physical Review Letters, 119 (5):054101, July 2017. doi: 10.1103/PhysRevLett.119.054101. URL https://link. aps.org/doi/10.1103/PhysRevLett.119.054101.

[89] J. R. Dorfman. An Introduction to Chaos in Nonequilibrium Statistical Mechanics. Cambridge University Press, Cambridge, England, 1999. ISBN 978-0-511-62887-0. URL http://ebooks . cambridge.org/ref/id/CB09780511628870.

[90] Alfonso Bueno-Orovio, Elizabeth M. Cherry, and Flavio H. Fenton. Minimal model for human ventricular action potentials in tissue. Journal of Theoretical Biology, 253 (3):544-560, August 2008. ISSN 00225193. doi: 10.1016/j.jtbi.2008.03.029. URL http://linkinghub.elsevier.com/retrieve/pii/S0022519308001690.

[91] C Morris and H Lecar. Voltage oscillations in the barnacle giant muscle fiber. Biophysical Journal, 35(1):193-213, July 1981. ISSN 0006-3495. URL http://www .ncbi. nlm.nih.gov/pmc/articles/PMC1327511/

[92] P. Gray and S.K. Scott. Autocatalytic reactions in the isothermal, continuous stirred tank reactor. Oscillations and instabilities in the system $\mathrm{A}+2 \mathrm{~b}->3 \mathrm{~b} ; \mathrm{B}->\mathrm{C}$. Chemical Engineering Science, 39(6):1087-1097, 1984. doi: 10.1016/0009-2509(84) 87017-7.

[93] Kaori Sugimura and Hiroshi Kori. Exponential system-size dependence of the lifetime of transient spiral chaos in excitable and oscillatory media. Physical Review E, 92(6): 062915, 2015. URL http://journals.aps.org/pre/abstract/10.1103/PhysRevE. 92.062915 .

[94] Matthew C. Strain and Henry S. Greenside. Size-dependent transition to highdimensional chaotic dynamics in a two-dimensional excitable medium. Physical Review Letters, 80(11):2306, 1998. URL http://journals .aps.org/prl/abstract/10. 1103/PhysRevLett.80.2306.

[95] Renate Wackerbauer and Kenneth Showalter. Collapse of Spatiotemporal Chaos. Physical Review Letters, 91(17):174103, October 2003. ISSN 0031-9007, 1079-7114. URL http://link.aps.org/doi/10.1103/PhysRevLett.91.174103.

[96] Keegan Keplinger and Renate Wackerbauer. Transient spatiotemporal chaos in the Morris-Lecar neuronal ring network. Chaos: An Interdisciplinary Journal of Nonlinear Science, 24(1):013126, March 2014. ISSN 1054-1500. doi: 10.1063/1.4866974. URL http://aip.scitation.org/doi/10.1063/1.4866974. 
[97] James P. Keener and James Sneyd. Mathematical physiology. Number 8 in Interdisciplinary applied mathematics. Springer, New York, NY, 2nd ed edition, 2009. ISBN 978-0-387-09419-9 978-0-387-75846-6 978-0-387-79387-0. OCLC: ocn298595247.

[98] Roy M. Smeal, G. Bard Ermentrout, and John A. White. Phase-response curves and synchronized neural networks. Philosophical Transactions of the Royal Society B: Biological Sciences, 365(1551):2407-2422, August 2010. ISSN 0962-8436. doi: 10.1098/ rstb.2009.0292. URL http://www.ncbi.nlm.nih.gov/pmc/articles/PMC2894948/.

[99] Susanne Still and Gwendal Le Masson. Traveling waves in a ring of three inhibitory coupled model neurons. Neurocomputing, 26:533-539, June 1999. ISSN 0925-2312. doi: 10.1016/S0925-2312(99)00031-4. URL http://www.sciencedirect.com/science/ article/pii/S0925231299000314.

[100] Hiroshi Fujii and Ichiro Tsuda. Neocortical gap junction-coupled interneuron systems may induce chaotic behavior itinerant among quasi-attractors exhibiting transient synchrony. Neurocomputing, 58:151-157, June 2004. ISSN 0925-2312. doi: 10.1016/ j.neucom.2004.01.036. URL http://www.sciencedirect.com/science/article/ pii/S0925231204000323.

[101] D. Allexandre and N. F. Otani. Preventing alternans-induced spiral wave breakup in cardiac tissue: an ion-channel-based approach. Physical Review. E, Statistical, Nonlinear, and Soft Matter Physics, 70(6 Pt 1):061903, December 2004. ISSN 15393755. doi: 10.1103/PhysRevE.70.061903.

[102] Peter J. Menck, Jobst Heitzig, Norbert Marwan, and Jürgen Kurths. How basin stability complements the linear-stability paradigm. Nature Physics, 9(2):89-92, January 2013. ISSN 1745-2473, 1745-2481. doi: 10.1038/nphys2516. URL http: //www.nature.com/doifinder/10.1038/nphys2516.

[103] Ying-Cheng Lai and Raimond L. Winslow. Geometric Properties of the Chaotic Saddle Responsible for Supertransients in Spatiotemporal Chaotic Systems. Physical Review Letters, 74(26):5208-5211, June 1995. doi: 10.1103/PhysRevLett.74.5208. URL https://link.aps.org/doi/10.1103/PhysRevLett.74.5208.

[104] Gerrit Ansmann, Klaus Lehnertz, and Ulrike Feudel. Self-Induced Switchings between Multiple Space-Time Patterns on Complex Networks of Excitable Units. Physical Review X, 6(1):011030, March 2016. ISSN 2160-3308. URL https://link.aps.org/ doi/10.1103/PhysRevX.6.011030.

[105] Michael Monteforte and Fred Wolf. Dynamic Flux Tubes Form Reservoirs of Stability in Neuronal Circuits. Physical Review X, 2(4):041007, November 2012. ISSN 21603308. URL https://link.aps.org/doi/10.1103/PhysRevX.2.041007.

[106] Antonio Politi and Alessandro Torcini. Stable Chaos. In Nonlinear Dynamics and Chaos: Advances and Perspectives, Understanding Complex Systems, page 103. 
Springer, Berlin, Heidelberg, 2010. ISBN 978-3-642-04628-5 978-3-642-04629-2. URL https://link.springer.com/chapter/10.1007/978-3-642-04629-2_6.

[107] Hinke M. Osinga. Boundary crisis bifurcation in two parameters. Journal of Difference Equations and Applications, 12(10):997-1008, October 2006. ISSN 1023-6198. doi: 10. 1080/10236190600986826. URL http://dx.doi.org/10.1080/10236190600986826.

[108] M. Santini, C. Pandozi, G. Altamura, G. Gentilucci, M. Villani, M. C. Scianaro, A. Castro, F. Ammirati, and B. Magris. Single shock endocavitary low energy intracardiac cardioversion of chronic atrial fibrillation. J. Interventional Cardiac Electrophysiology: An International Journal of Arrhythmias and Pacing, 3(1):45-51, March 1999. ISSN 1383-875X.

[109] R.H. Clayton, A. Murray, P.D. Higham, and R.W.F. Campbell. Self-terminating ventricular tachyarrhythmias - a diagnostic dilemma? The Lancet, 341(8837):p.9395, January 1993. ISSN 01406736. doi: 10.1016/0140-6736(93)92567-D. URL http: //linkinghub.elsevier.com/retrieve/pii/014067369392567D.

[110] Mikhail I. Rabinovich and Pablo Varona. Robust Transient Dynamics and Brain Functions. Frontiers in Computational Neuroscience, 5:24, June 2011. ISSN 16625188. URL http://www.ncbi.nlm.nih.gov/pmc/articles/PMC3116137/.

[111] C. H. Luo and Y. Rudy. A model of the ventricular cardiac action potential. Depolarization, repolarization, and their interaction. Circulation Research, 68(6):1501-1526, June 1991. ISSN 0009-7330.

[112] Richard A. Gray, Arkady M. Pertsov, and José Jalife. Spatial and temporal organization during cardiac fibrillation. Nature, 392(6671):75-78, March 1998. ISSN 00280836. doi: 10.1038/32164. URL http://www.nature.com/doifinder/10.1038/32164.

[113] Galen S. Wagner and Henry D. McIntosh. The use of drugs in achieving successful DC cardioversion. Progress in Cardiovascular Diseases, 11(5):431-442, 1969. ISSN 0033-0620. doi: 10.1016/0033-0620(69)90030-9. URL http://www.sciencedirect. com/science/article/pii/0033062069900309.

[114] Matthew J. Reed, Gareth R. Clegg, and Colin E. Robertson. Analysing the ventricular fibrillation waveform. Resuscitation, 57(1):11-20, April 2003. ISSN 03009572. doi: 10.1016/S0300-9572(02)00441-0. URL http://www.sciencedirect.com/ science/article/pii/S0300957202004410.

[115] Douglas L. Jones and George J. Klein. Ventricular fibrillation: The importance of being coarse? Journal of Electrocardiology, 17(4):393-399, January 1984. ISSN 00220736. doi: 10.1016/S0022-0736(84)80077-1. URL http://www.sciencedirect.com/ science/article/pii/S0022073684800771.

[116] P. S. Addison, J. N. Watson, G. R. Clegg, M. Holzer, F. Sterz, and C. E. Robertson. Evaluating arrhythmias in ECG signals using wavelet transforms. IEEE Engineering 
in Medicine and Biology Magazine, 19(5):104-109, September 2000. ISSN 0739-5175. doi: $10.1109 / 51.870237$.

[117] José Amigó. Permutation Complexity in Dynamical Systems. Springer Series in Synergetics. Springer Berlin Heidelberg, Berlin, Heidelberg, 2010. ISBN 978-3-642-04083-2 978-3-642-04084-9. URL http://link.springer.com/10.1007/ 978-3-642-04084-9.

[118] U Parlitz, S Berg, S Luther, A Schirdewan, J Kurths, and N Wessel. Classifying cardiac biosignals using ordinal pattern statistics and symbolic dynamics. Comput. Biol. Med., 42(3):319-327, March 2012. ISSN 1879-0534. doi: 10.1016/j.compbiomed.2011.03.017.

[119] Christoph Bandt and Bernd Pompe. Permutation entropy: a natural complexity measure for time series. Physical Review Letters, 88(17):174102, April 2002. ISSN 0031-9007. doi: 10.1103/PhysRevLett.88.174102.

[120] F. Daviaud, M. Bonetti, and M. Dubois. Transition to turbulence via spatiotemporal intermittency in one-dimensional Rayleigh-Bénard convection. Physical Review A, 42(6):3388, 1990. URL http://journals .aps .org/pra/abstract/10.1103/ PhysRevA.42.3388.

[121] Jerry P. Gollub. Order and disorder in fluid motion. Proceedings of the National Academy of Sciences, 92(15):6705-6711, 1995. URL http://www.pnas .org/content/ 92/15/6705. short.

[122] Kunihiko Kaneko. Theory and applications of coupled map lattices. Wiley, Chichester : New York, 1993. ISBN 978-0-471-93741-8.

[123] Peter Rupp, Reinhard Richter, and Ingo Rehberg. Critical exponents of directed percolation measured in spatiotemporal intermittency. Physical Review E, 67(3), March 2003. ISSN 1063-651X, 1095-3787. doi: 10.1103/PhysRevE.67.036209. URL http://link.aps.org/doi/10.1103/PhysRevE.67.036209.

[124] Peter JA Bollen, Axel K Hansen, and Aage K Olsen Alstrup. The laboratory swine. CRC Press, 2010.

[125] B. Podesser, G. Wollenek, R. Seitelberger, H. Siegel, E. Wolner, W. Firbas, and M. Tschabitscher. Epicardial branches of the coronary arteries and their distribution in the rabbit heart: the rabbit heart as a model of regional ischemia. The Anatomical Record, 247(4):521-527, April 1997. ISSN 0003-276X.

[126] Flavio H. Fenton, Stefan Luther, Elizabeth M. Cherry, Niels F. Otani, Valentin Krinsky, Alain Pumir, Eberhard Bodenschatz, and Robert F. Gilmour. Termination of Atrial Fibrillation Using Pulsed Low-Energy Far-Field Stimulation. Circulation, 120(6):467-476, August 2009. ISSN 0009-7322, 1524-4539. doi: 10.1161/ CIRCULATIONAHA.108.825091. URL http://circ.ahajournals.org/content/ $120 / 6 / 467$. 
[127] R. Plonsey. The nature of sources of bioelectric and biomagnetic fields. Biophysical Journal, 39(3):309-312, September 1982. ISSN 00063495. doi: 10.1016/ S0006-3495(82)84521-9. URL http://linkinghub.elsevier.com/retrieve/pii/ S0006349582845219.

[128] Ulrich Parlitz, Alexander Schlemmer, and Stefan Luther. Synchronization patterns in transient spiral wave dynamics. Physical Review E, 83(5), May 2011. ISSN 1539-3755. doi: 10.1103/PhysRevE.83.057201.

[129] Gernot Plank, L. Joshua Leon, Shane Kimber, and Edward J. Vigmond. Defibrillation depends on conductivity fluctuations and the degree of disorganization in reentry patterns. Journal of Cardiovascular Electrophysiology, 16(2):205-216, February 2005. ISSN 1045-3873. doi: 10.1046/j.1540-8167.2005.40140.x.

[130] Sean P. Cornelius, William L. Kath, and Adilson E. Motter. Realistic control of network dynamics. Nature Communications, 4:ncomms2939, June 2013. ISSN 2041-1723. doi: 10.1038/ncomms2939. URL https://www .nature.com/articles/ncomms2939.

[131] Greg Byrne, Christopher D. Marcotte, and Roman O. Grigoriev. Exact coherent structures and chaotic dynamics in a model of cardiac tissue. Chaos (Woodbury, N.Y.), 25(3):033108, March 2015. ISSN 1089-7682. doi: 10.1063/1.4915143.

[132] Claudia Lerma and Leon Glass. Predicting the risk of sudden cardiac death. The Journal of Physiology, 594(9):2445-2458, May 2016. ISSN 1469-7793. doi: 10.1113/ JP270535.

[133] James M. McLenachan, Esther Henderson, Karen I. Morris, and Henry J. Dargie. Ventricular Arrhythmias in Patients with Hypertensive Left Ventricular Hypertrophy. New England Journal of Medicine, 317(13):787-792, September 1987. ISSN 0028-4793, 1533-4406. doi: 10.1056/NEJM198709243171302. URL http://www.nejm.org/doi/ abs/10.1056/NEJM198709243171302.

[134] T. Kahan. Left ventricular hypertrophy in hypertension: its arrhythmogenic potential. Heart, 91(2):250-256, February 2005. ISSN 1355-6037. doi: 10.1136/hrt.2004.042473. URL http://heart.bmj . com/cgi/doi/10.1136/hrt.2004.042473.

[135] Anil Verma, Alessandra Meris, Hicham Skali, Jalal K. Ghali, J. Malcolm O. Arnold, Mikhail Bourgoun, Eric J. Velazquez, John J.V. McMurray, Lars Kober, Marc A. Pfeffer, Robert M. Califf, and Scott D. Solomon. Prognostic Implications of Left Ventricular Mass and Geometry Following Myocardial Infarction. JACC: Cardiovascular Imaging, 1(5):582-591, September 2008. ISSN 1936878X. doi: 10.1016/j.jcmg.2008.05.012. URL http://linkinghub.elsevier .com/retrieve/pii/S1936878X0800226X.

[136] M. Yamazaki, H. Honjo, H. Nakagawa, Y. S. Ishiguro, Y. Okuno, M. Amino, I. Sakuma, K. Kamiya, and I. Kodama. Mechanisms of destabilization and early termination of spiral wave reentry in the ventricle by a class III antiarrhythmic 
agent, nifekalant. AJP: Heart and Circulatory Physiology, 292(1):H539-H548, October 2006. ISSN 0363-6135, 1522-1539. doi: 10.1152/ajpheart.00640.2006. URL http://ajpheart.physiology.org/cgi/doi/10.1152/ajpheart.00640.2006.

[137] R. A. Winkle, E. B. Stinson, S. M. Bach, D. S. Echt, P. Oyer, and K. Armstrong. Measurement of cardioversion/defibrillation thresholds in man by a truncated exponential waveform and an apical patch-superior vena caval spring electrode configuration. Circulation, 69(4):766-771, April 1984. ISSN 0009-7322.

[138] John E. Pearson. Complex Patterns in a Simple System. Science, 261(5118):189192, July 1993. ISSN 0036-8075, 1095-9203. doi: 10.1126/science.261.5118.189. URL http://science.sciencemag.org/content/261/5118/189.

[139] Richard H. Clayton. Vortex filament dynamics in computational models of ventricular fibrillation in the heart. Chaos: An Interdisciplinary Journal of Nonlinear Science, 18 (4):043127, 2008. ISSN 10541500. doi: 10.1063/1.3043805. URL http://scitation. aip.org/content/aip/journal/chaos/18/4/10.1063/1.3043805

[140] C. F. Starmer, D. N. Romashko, R. S. Reddy, Y. I. Zilberter, J. Starobin, A. O. Grant, and V. I. Krinsky. Proarrhythmic Response to Potassium Channel Blockade Numerical Studies of Polymorphic Tachyarrhythmias. Circulation, 92(3):595605, August 1995. ISSN 0009-7322, 1524-4539. doi: 10.1161/01.CIR.92.3.595. URL http://circ.ahajournals.org/content/92/3/595.

[141] Marc Courtemanche and Arthur T. Winfree. Re-entrant rotating waves in a beeler-reuter based model of two-dimensional cardiac electrical activity. International Journal of Bifurcation and Chaos, 01(2):431-444, June 1991. ISSN 02181274. doi: 10.1142/S0218127491000336. URL http://www.worldscientific.com/ doi/abs/10.1142/S0218127491000336.

[142] L. J. Leon, F. A. Roberge, and A. Vinet. Simulation of two-dimensional anisotropic cardiac reentry: effects of the wavelength on the reentry characteristics. Annals of biomedical engineering, 22(6):592-609, 1994. URL http://link.springer.com/ article/10.1007/BF02368286.

[143] Igor R. Efimov, Valentin I. Krinsky, and Jose Jalife. Dynamics of rotating vortices in the Beeler-Reuter model of cardiac tissue. Chaos, Solitons 85 Fractals, 5(3):513526, March 1995. ISSN 0960-0779. doi: 10.1016/0960-0779(95)95761-F. URL http: //www.sciencedirect.com/science/article/pii/096007799595761F.

[144] Marc Courtemanche. Complex spiral wave dynamics in a spatially distributed ionic model of cardiac electrical activity. Chaos: An Interdisciplinary Journal of Nonlinear Science, 6(4):579-600, December 1996. ISSN 1054-1500, 1089-7682. doi: 10.1063/1. 166206. URL http://scitation.aip.org/content/aip/journal/chaos/6/4/10. 1063/1.166206. 
[145] Aoxiang Xu and Michael R. Guevara. Two forms of spiral-wave reentry in an ionic model of ischemic ventricular myocardium. Chaos: An Interdisciplinary Journal of Nonlinear Science, 8(1):157-174, March 1998. ISSN 1054-1500, 1089-7682. doi: 10. 1063/1.166286. URL http://scitation.aip.org/content/aip/journal/chaos/8/ $1 / 10.1063 / 1.166286$.

[146] Robin M. Shaw and Yoram Rudy. Electrophysiologic Effects of Acute Myocardial Ischemia A Mechanistic Investigation of Action Potential Conduction and Conduction Failure. Circulation Research, 80(1):124-138, January 1997. ISSN 0009-7330, 1524-4571. doi: 10.1161/01.RES.80.1.124. URL http://circres . ahajournals .org/ content/80/1/124.

[147] Olivier Bernus, Christian W. Zemlin, Roman M. Zaritsky, Sergey F. Mironov, and Arkady M. Pertsov. Alternating conduction in the ischaemic border zone as precursor of reentrant arrhythmias: A simulation study. Europace, 7(s2):S93-S104, January 2005. ISSN 1099-5129, 1532-2092. doi: 10.1016/j.eupc.2005.03.018. URL http: //europace.oxfordjournals.org/content/7/s2/S93.

[148] A. Schlemmer, T. Baig, S. Luther, and U. Parlitz. Detection and characterization of intermittent complexity variations in cardiac arrhythmia. Physiol. Meas., 38(8):1561, 2017. ISSN 0967-3334. doi: 10.1088/1361-6579/aa7be0. URL http://stacks .iop. org $/ 0967-3334 / 38 / i=8 / a=1561$.

[149] Andrei V. Sazonov, Chin Keong Ho, Jan W. M. Bergmans, Johan B. A. M. Arends, Paul A. M. Griep, Evgeny A. Verbitskiy, Pierre J. M. Cluitmans, and Paul A. J. M. Boon. An investigation of the phase locking index for measuring of interdependency of cortical source signals recorded in the EEG. Biological Cybernetics, 100(2):129146, February 2009. ISSN 0340-1200. doi: 10.1007/s00422-008-0283-4. URL https: //www.ncbi.nlm.nih.gov/pmc/articles/PMC2792353/

[150] Scott David Greenwald. The development and analysis of a ventricular fibrillation detector. Thesis, Massachusetts Institute of Technology, 1986. URL http://dspace. mit.edu/handle/1721.1/92988.

[151] Ary L. Goldberger, Luis A. N. Amaral, Leon Glass, Jeffrey M. Hausdorff, Plamen Ch Ivanov, Roger G. Mark, Joseph E. Mietus, George B. Moody, Chung-Kang Peng, and H. Eugene Stanley. PhysioBank, PhysioToolkit, and PhysioNet. Circulation, 101(23): e215-e220, June 2000. ISSN 0009-7322, 1524-4539. doi: 10.1161/01.CIR.101.23.e215. URL http://circ . ahajournals .org/content/101/23/e215.

[152] Cynthia M. Tracy, Andrew E. Epstein, Dawood Darbar, John P. DiMarco, Sandra B. Dunbar, N.A. Mark Estes, T. Bruce Ferguson, Stephen C. Hammill, Pamela E. Karasik, Mark S. Link, Joseph E. Marine, Mark H. Schoenfeld, Amit J. Shanker, Michael J. Silka, Lynne Warner Stevenson, William G. Stevenson, and Paul D. Varosy. 2012 ACCF/AHA/HRS Focused Update Incorporated Into the ACCF/AHA/HRS 
2008 Guidelines for Device-Based Therapy of Cardiac Rhythm Abnormalities. Journal of the American College of Cardiology, 61(3):e6-e75, January 2013. ISSN 07351097. doi: 10.1016/j.jacc.2012.11.007. URL http://linkinghub.elsevier.com/ retrieve/pii/S0735109712054952

[153] Christian Kupatt, Rabea Hinkel, Jan Horstkotte, Michael Deiß, Marie-Luise von Brühl, Manfred Bilzer, and Peter Boekstegers. Selective retroinfusion of GSH and cariporide attenuates myocardial ischemia-reperfusion injury in a preclinical pig model. Cardiovascular Research, 61(3):530-537, February 2004. ISSN 0008-6363, 1755-3245. doi: 10.1016/j.cardiores.2003.11.012. URL http://cardiovascres. oxfordjournals.org/content/61/3/530. 


\section{Acknowledgements}

\section{Acknowledgements}

At this point, I would like to thank the people and organizations which supported me during the time of my PhD.

Foremost, I want to give many thanks to my supervisor apl. Professor Dr. Ulrich Parlitz, for introducing me into the exciting and manifold world of nonlinear dynamics. Thank you for your terrific support during my $\mathrm{PhD}$, in terms of so many scientific and non-scientific discussions at literally any time.

Many thanks go to my group leader Professor Dr. Stefan Luther for giving me the great opportunity to work in this exciting field. I am particularly grateful for providing the opportunities to discuss scientific results on many conferences.

Also, I thank my Thesis Committee Professor Dr. Andreas Tilgner, Dr. Florian Rehfeldt, and Professor Dr. Christoph Schmidt for guiding my $\mathrm{PhD}$ and providing valuable feedback during the Thesis Committee Meetings.

This work would not have been possible without the ongoing exchange and fruitful cooperations with my colleagues of the Research Group Biomedical Physics. I am grateful for the exceptionally open and friendly atmosphere in our group, which provided the basis for so many fruitful discussions of scientific questions but also for spending "non-scientific" time together. Particular thanks go to Sebastian Berg for charing his impressive skills in programming and numerics, Dr. Claudia Richter and Dr. Jan Christoph for significantly increasing my motivation at any time, and Dr. Alexander Schlemmer for the fruitful cooperation which benefited from our shared enthusiasm for scientific investigations.

Beyond the Research Group Biomedical Physics, I would like to thank Angela Meister for the administrative support and all colleagues of the Max Planck Institute for Dynamics and Self-Organization who accompanied me during the time of my PhD. Thanks go to Dr. Isabella Guido, Dr. Laura Turco, and Dr. Christian Westendorf for having such a great time, including a couple of coffees.

This thesis is mainly based on extensive numerical studies which were performed on the computer cluster of our group. For managing all kinds of technical challenges I thank in particular Dr. Hendrik "Hecke" Degering, Dr. Denny Fliegner and Gerhard Nolte.

I am grateful for the support through the International Max Planck Research School (IMPRS) for Physics of Biological and Complex Systems (PBCS) which funded my work via the IMPRS stipend and facilitated by their financial contribution the visit of diverse scientific 
conferences. In particular, special thanks go to Frauke Bergmann and Antje Erdmann who answered my continuous stream of questions with great patience and kindness at any time. Also, thanks to Daniel Nacke and the "Bielefeld Physics Connection" (Carolin Böger, Jan Haskenhoff, Dr. Torsten Hübner, Robin Klett, Oliver Reimer, Dr. Matthias Simonis, Michael Stührenberg, Hanno Meyer zu Theenhausen), who had a great impact on me as a person and as a physicist during the last years. Thank you for having such great friends.

Although my family was not involved scientifically in my work, they unequivocally contributed significantly by heartening me during difficult times and provided the perfect environment to regain energy. Thanks for this unconditional support and the encouragement to follow my scientific and non-scientific goals in life. 